\title{
International Union of Basic and Clinical Pharmacology. LXXXIV: Leukotriene Receptor Nomenclature, Distribution, and Pathophysiological Functions
}

Magnus Bäck, Sven-Erik Dahlén, Jeffrey M. Drazen, Jilly F. Evans, Charles N. Serhan, Takao Shimizu, Takehiko Yokomizo, and G. Enrico Rovati

Department of Medicine, Karolinska Institutet and Department of Cardiology, Karolinska University Hospital, Stockholm, Sweden (M.B.); The National Institute of Environmental Medicine, Division of Physiology, Karolinska Institutet, Stockholm, Sweden (S.-E.D.); Harvard Medical School, Brigham and Women's Hospital, Boston, Massachusetts (J.M.D.); Amira Pharmaceuticals, Inc., San Diego, California (J.F.E.); Center for Experimental Therapeutics and Reperfusion Injury, Brigham and Women's Hospital, Avenue Louis Pasteur, Boston, Massachusetts (C.N.S.); Department of Biochemistry and Molecular Biology, Faculty of Medicine, the University of Tokyo, Bunkyo-ku, Hongo, Tokyo, Japan (T.S.); Department of Medical Biochemistry, Kyushu University, Fukuoka, Japan (T.Y.); and Laboratory of Molecular Pharmacology, Department of Pharmacological Sciences, University of Milan, Milan, Italy (G.E.R.)

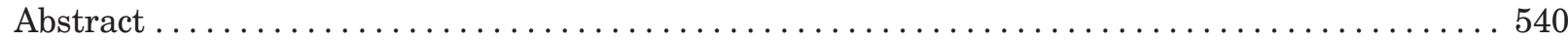

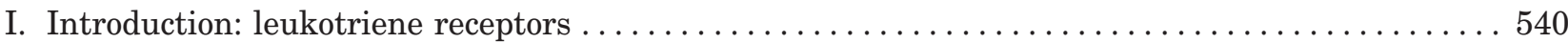

A. Brief historical background of leukotrienes and their receptors $\ldots \ldots \ldots \ldots \ldots \ldots \ldots \ldots 541$

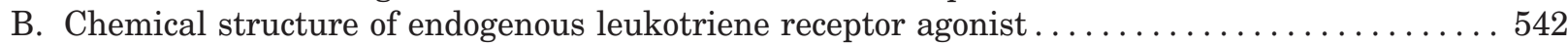

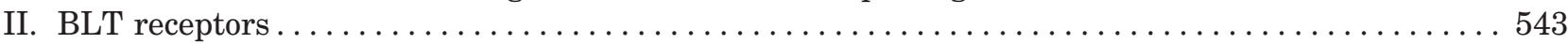

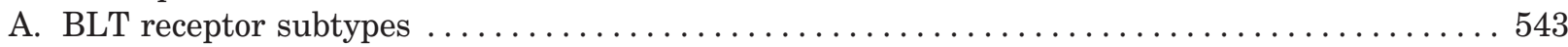

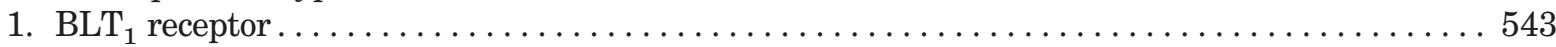

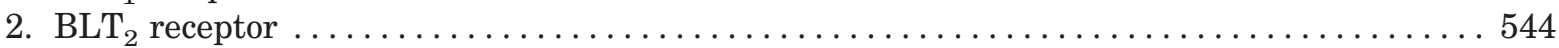

3. Genomic organization and transcriptional regulation $\ldots \ldots \ldots \ldots \ldots \ldots \ldots \ldots \ldots 4$

B. Structure-function relationships for BLT receptors $\ldots \ldots \ldots \ldots \ldots \ldots \ldots \ldots \ldots \ldots \ldots \ldots 45$

C. Intracellular signaling pathways and second-messenger systems $\ldots \ldots \ldots \ldots \ldots \ldots \ldots \ldots . \ldots 546$

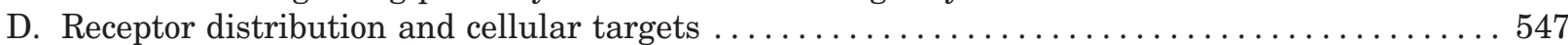

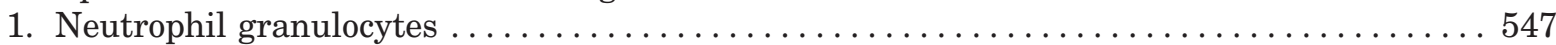

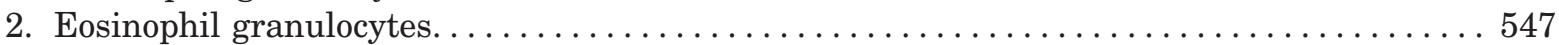

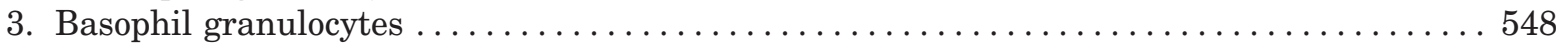

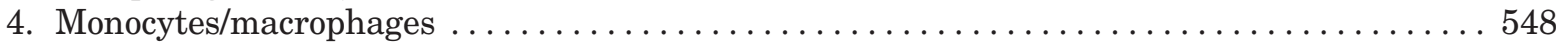

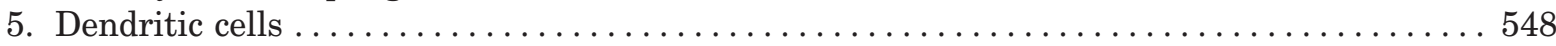

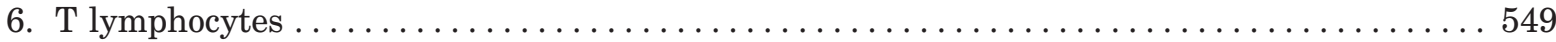

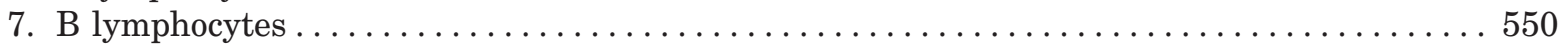

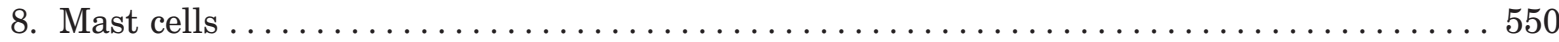

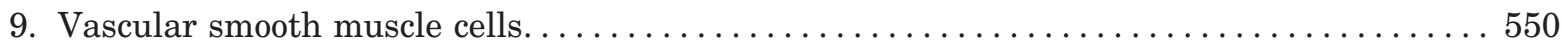

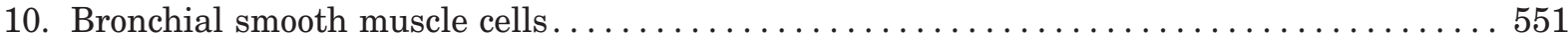

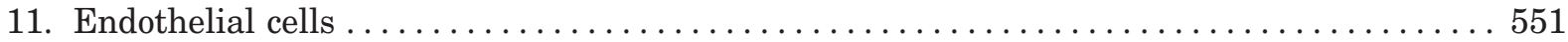

E. BLT receptor functional analysis through altered gene expression $\ldots \ldots \ldots \ldots \ldots \ldots \ldots \ldots 51$

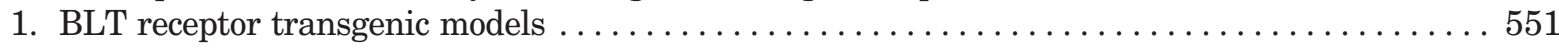

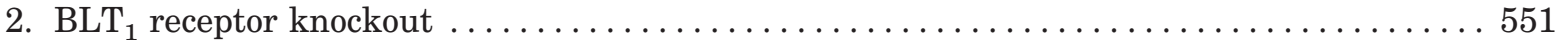

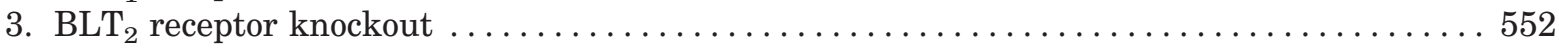

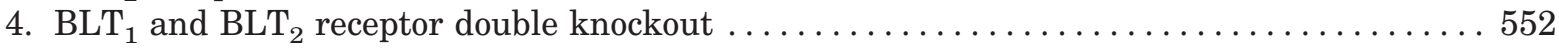

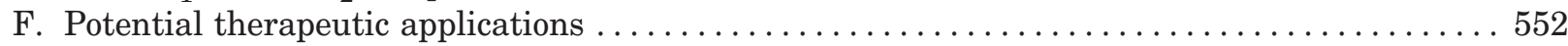

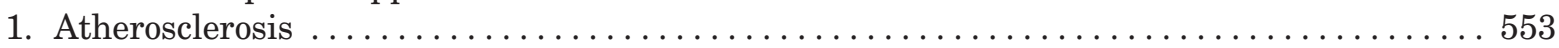

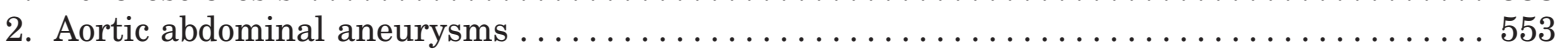

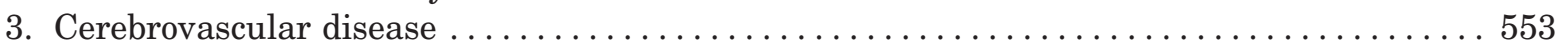

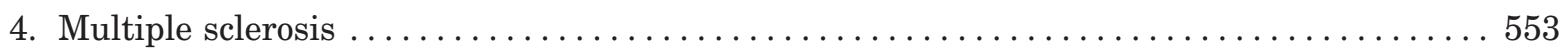

Address correspondence to: Dr. Magnus Bäck (Chairman), Karolinska University Hospital, Center for Molecular Medicine, L8:03, 17176 Stockholm, Sweden. E-mail: magnus.back@ki.se.

This article is available online at http://pharmrev.aspetjournals.org.

doi:10.1124/pr.110.004184. 


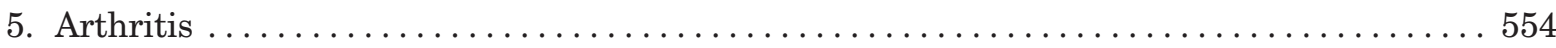

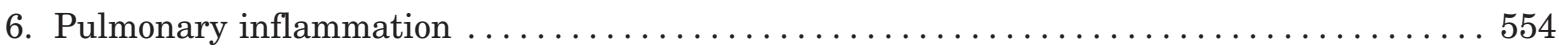

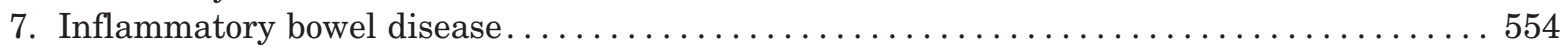

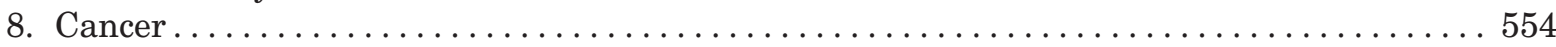

G. Other receptors involved in BLT receptor signaling $\ldots \ldots \ldots \ldots \ldots \ldots \ldots \ldots \ldots \ldots \ldots 4$

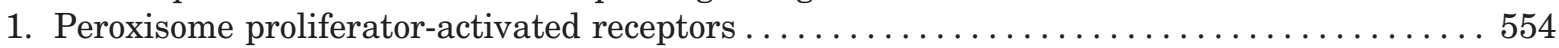

2. Vanilloid transient receptor potential V1 receptor $\ldots \ldots \ldots \ldots \ldots \ldots \ldots \ldots \ldots \ldots \ldots$

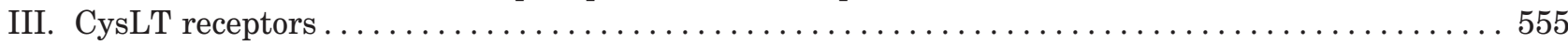

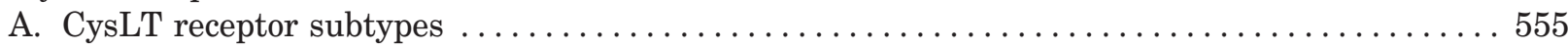

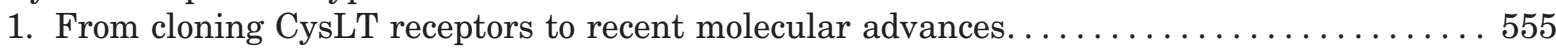

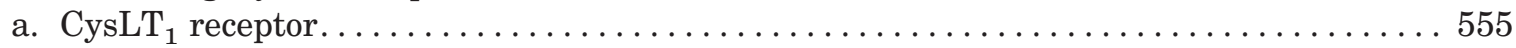

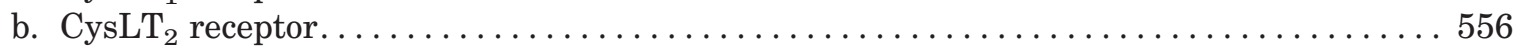

2. Receptor expression patterns with functional significance $\ldots \ldots \ldots \ldots \ldots \ldots \ldots \ldots \ldots 5$

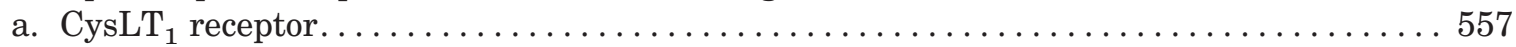

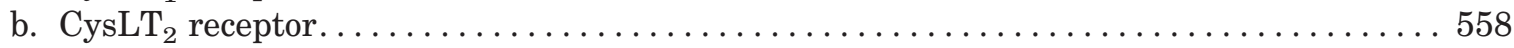

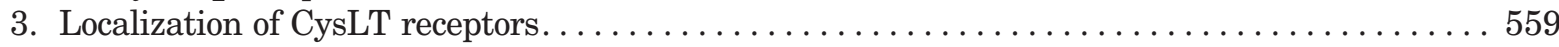

B. CysLT receptor expression and immunoregulation $\ldots \ldots \ldots \ldots \ldots \ldots \ldots \ldots \ldots \ldots \ldots \ldots$

C. Intracellular signaling pathways and second-messenger systems $\ldots \ldots \ldots \ldots \ldots \ldots \ldots \ldots 61$

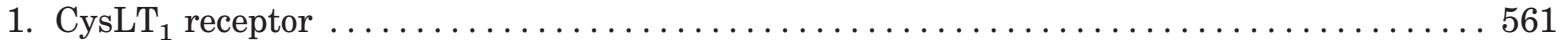

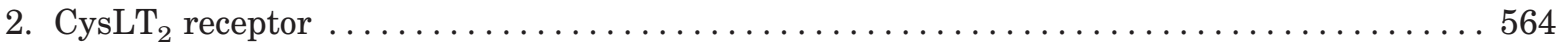

D. Desensitization and cross-talk of CysLT receptors ......................... 564

E. CysLT receptor functional analysis through altered gene expression $\ldots \ldots \ldots \ldots \ldots \ldots \ldots 56$

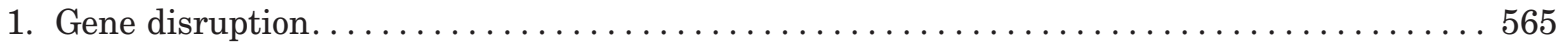

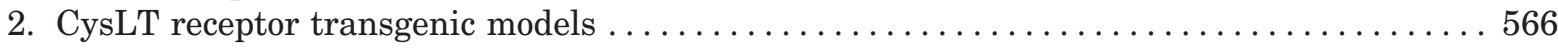

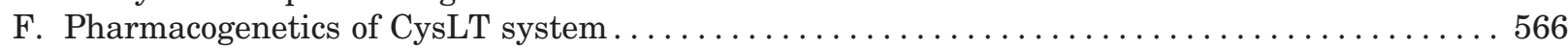

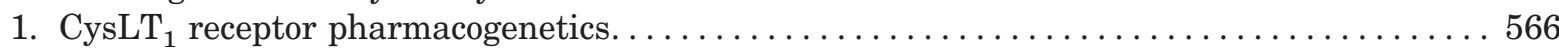

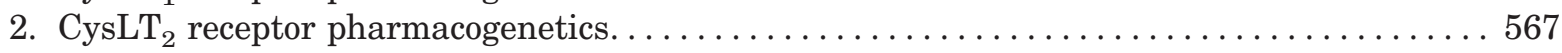

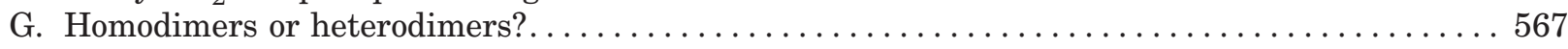

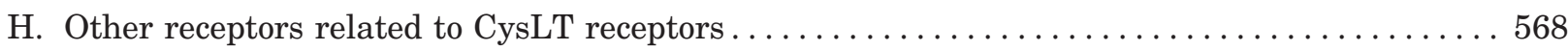

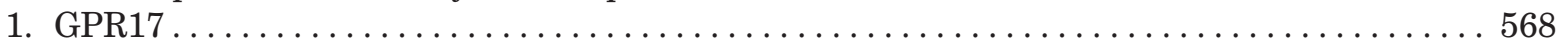

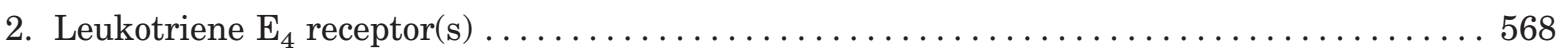

I. New potential therapeutic applications of leukotriene receptor antagonists $\ldots \ldots \ldots \ldots \ldots 569$

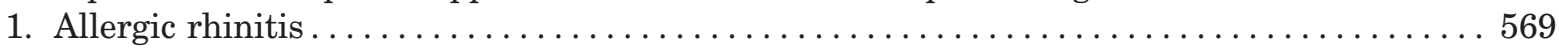

2. Aspirin/nonsteroidal anti-inflammatory drug intolerance $\ldots \ldots \ldots \ldots \ldots \ldots \ldots \ldots \ldots 9$

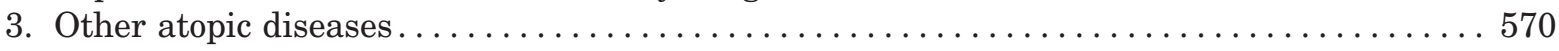

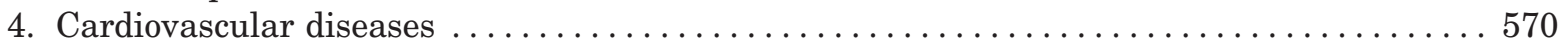

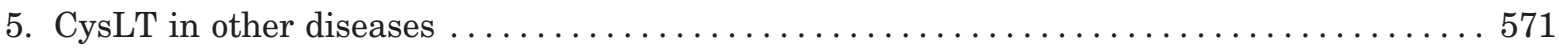

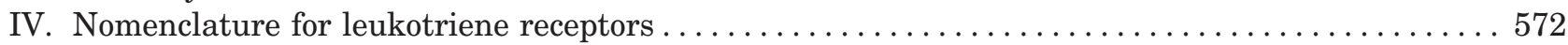

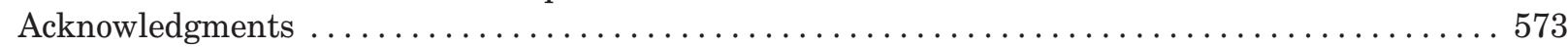

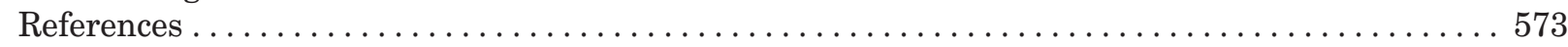

\begin{abstract}
The seven-transmembrane G proteincoupled receptors activated by leukotrienes are divided into two subclasses based on their ligand specificity for either leukotriene $B_{4}$ or the cysteinyl leukotrienes $\left(\mathrm{LTC}_{4}, \mathrm{LTD}_{4}\right.$, and $\left.\mathrm{LTE}_{4}\right)$. These receptors have been designated BLT and CysLT receptors, respectively, and a subdivision into $\mathrm{BLT}_{1}$ and $\mathrm{BLT}_{2}$ receptors and $\mathrm{CySLT}_{1}$ and $\mathrm{CysLT}_{2}$ receptors has been established. However, recent findings have also indicated the existence of pu-
\end{abstract}

tative additional leukotriene receptor subtypes. Furthermore, other ligands interact with the leukotriene receptors. Finally, leukotrienes may also activate other receptor classes, such as purinergic receptors. The aim of this review is to provide an update on the pharmacology, expression patterns, and pathophysiological roles of the leukotriene receptors as well as the therapeutic developments in this area of research.

\section{Introduction: Leukotriene Receptors}

The seven-transmembrane $G$ protein-coupled receptors $\left(\mathrm{GPCRs}^{1}\right)$ activated by leukotrienes are divided into two subclasses based on their ligand specificity for either leukotriene $\mathrm{B}_{4}\left(\mathrm{LTB}_{4}\right)$ or the cysteinyl leukotrienes $\left(\mathrm{LTC}_{4}, \mathrm{LTD}_{4}\right.$, and $\left.\mathrm{LTE}_{4}\right)$. The leukotriene receptors belong to the A5 subfamily of the rhodopsin receptor-like GPCRs (Joost and Methner, 2002). According to the 
previous report from the International Union of Basic and Clinical Pharmacology's (IUPHAR) Nomenclature Committee for Leukotriene Receptors, these receptors have been designated BLT and CysLT receptors, respectively (Brink et al., 2003). Furthermore, a subdivision into $\mathrm{BLT}_{1}$ and $\mathrm{BLT}_{2}$ receptors and $\mathrm{CysLT}_{1}$ and $\mathrm{CysLT}_{2}$ receptors has been established, as indicated in Table 1 (Brink et al., 2003).

However, several reports have also indicated the existence of additional leukotriene receptor subtypes (Rovati et al., 1997; Bäck, 2002; Norel and Brink, 2004; Rovati and Capra, 2007; Austen et al., 2009). In addition, evidence has emerged that ligands other than leukotrienes interact with the leukotriene receptors and

\footnotetext{
${ }^{1}$ Abbreviations: $\beta_{2} \mathrm{AR}, \beta_{2}$-adrenoreceptor(s); aa, amino acids; $\mathrm{AD}$, atopic dermatitis; AIA, aspirin-induced asthma; AML1, acute myeloid leukemia 1; AP-1, activator protein-1; AR, allergic rhinitis; ATA, aspirin-tolerant asthma; BAL, broncho-alveolar lavage; BAY U9773, 6(R)(4-carboxyphenylthio)-5(S)-hydroxy-7( $E), 9(E), 11(Z), 14(Z)$-eicosatetraenoic acid; BLTR, BLT receptor(s); $\mathrm{BLT}_{1} \mathrm{R}, \mathrm{BLT}_{1}$ receptor(s); $\mathrm{BLT}_{2} \mathrm{R}$, $\mathrm{BLT}_{2}$ receptor(s); $\mathrm{BMDC}$, bone marrow-derived dendritic cell; $\mathrm{BMMC}$, bone marrow-derived mast cell; $\mathrm{CF}$, cystic fibrosis; $\mathrm{CHO}$, Chinese hamster ovary; COPD, chronic obstructive pulmonary disease; COX, cyclooxygenase; CP-105,696, 1-[3-(4-phenyl-benzyl)-4-hydroxy-chroman-7yl] cyclopentane carboxylic acid; CU, chronic urticaria; CVD, cardiovascular disease; CysLTR, cysteinyl leukotriene receptor(s); $\operatorname{CysLT}_{1} R, \operatorname{CysLT}_{1}$ receptor(s); $\operatorname{CysLT}_{2} \mathrm{R}, \mathrm{CysLT}_{2}$ receptor(s); DC, dendritic cell; DSS, dextran sodium sulfate; EAE, allergic encephalomyelitis; EC, endothelial cell; ECP, eosinophil cationic protein; EGFR, epidermal growth factor receptor; ERK, extracellular signal-regulated kinases; FcR, Fc receptor; FLAP, 5-lipoxygenase activating protein; GPCR, G protein-coupled receptor; GRK, GPCR kinases; h, human; HEK, human embryonic kidney; HETE, hydroxyeicosatetraenoic acid; HHT, hydroxyheptadecatrienoic acid; HpETE, hydroperoxyeicosatetraenoic acid; HUVEC, human umbilical cord endothelial cell; IBD, inflammatory bowel disease; IFN, interferon; IGF, insulin growth factor; IL, interleukin; IUPHAR, International Union of Basic and Clinical Pharmacology; JNK, c-Jun $\mathrm{NH}_{2}$-terminal kinase; 5-LO, 5-lipoxygenase; LPS, lipopolysaccharide; LT, leukotriene; LTC4S, LTC 4 synthase; LTRA, leukotriene receptor antagonist; LY255283, 1-[5-ethyl-2hydroxy-4-[[6-methyl-6-(1H-tetrazol-5-yl)heptyl]oxy]phenyl]ethanone; LY293111, 2-[2-propyl-3-[3-[2-ethyl-4-(4-fluorophenyl)-5-hydroxyphenoxy]-propoxy]phenoxyl]benzoic acid; m, murine; MAPK, mitogenactivated protein kinase; MC, mast cell; MCP, monocyte chemoattractant protein; MIP, macrophage inflammatory protein; MK0571, 3-[[3-[2-(7-chloroquinolin-2-yl)vinyl]phenyl]-(2-dimethylcarbamoylethylsulfanyl)methylsulfanyl] propionic acid; MMP, matrix metalloproteinases; MNC, mononuclear cell; MRS 2395, 2,2-dimethyl-propionic acid 3-(2-chloro-6-methylaminopurin-9-yl)-2-(2,2-dimethyl-propionyloxymethyl)-propyl ester; MS, multiple sclerosis; NCBI, National Center for Biotechnology Information; NF- $\kappa \mathrm{B}$, nuclear factor $\kappa \mathrm{B}$; NO, nitric oxide; NSAID, nonsteroidal anti-inflammatory drug; OVA, ovalbumin; $\mathrm{P} 2 \mathrm{Y}$, pyrimidinergic nucleotide; $\mathrm{PAF}$, platelet-activating factor; $\mathrm{PGE}_{2}$, prostaglandin $\mathrm{E}_{2}$; PI, phosphatidylinositol; PI3K, phosphatidylinositol 3-kinase; PK, protein kinase; PPAR, peroxisome proliferator-activated receptor; PTX, pertussis toxin; QL, quality of life; RANTES, regulated on activation normal $\mathrm{T}$ cell expressed and secreted; RT-PCR, reverse transcriptase-polymerase chain reaction; Rv, resolvin; SKF 104353, 2(S)-hydroxyl-3(R)-carboxyethylthio)-3-[2-(8-phenyloctyl) phenyl] propanoic acid; SMC, smooth muscle cell; SNP, single nucleotide polymorphism; STAT, signal transducer and activator of transcription; Th, T helper; TM, transmembrane domain; TNF, tumor necrosis factor; TRP, transient receptor potential; U75302, -[6-(3-hydroxy-1E,5Z-undecadienyl)-2-pyridinyl]-1,5-hexanediol; VEGF, vascular endothelial growth factor; WT, wild type.
}

that leukotrienes may also signal through receptors preferentially activated by other endogenous ligands. For example, lipid mediators that are structurally different from leukotrienes have been reported to signal through BLT receptors (Arita et al., 2007; Okuno et al., 2008). As described later, 12(S)-hydroxyheptadeca$5 Z, 8 E, 10 E$-trienoic acid (12-HHT) was identified as a more potent ligand for $\mathrm{BLT}_{2} \mathrm{R}$ than for $\mathrm{LTB}_{4}$ (Okuno et al., 2008). Furthermore, although evidence that $\mathrm{LTE}_{4}$ signals through the $\mathrm{P}_{2} \mathrm{Y}_{12}$ receptor was demonstrated (Nonaka et al., 2005; Paruchuri et al., 2009; Fredman et al., 2010), one of the orphan GPCRs, GPR17, has been postulated to be activated by both cysteinyl leukotrienes and nucleotides (Ciana et al., 2006). Finally, both the CysLT $_{1}$ receptor $\left(\mathrm{CysLT}_{1} \mathrm{R}\right)$ antagonists in clinical use as antiasthmatic drugs have been demonstrated to inhibit the effects of nucleotides acting at different P2Y receptors. These observations demonstrate that these classic leukotriene receptor antagonists (LTRAs) can inhibit non-CysLT ${ }_{1}$-mediated proinflammatory reactions, suggesting activities potentially relevant for interpatient variability in response to treatment. The aim of the present review is to provide an extensive update on the pharmacology, expression patterns, and pathophysiological roles of the leukotriene receptors. Furthermore, a comment on the potential therapeutic developments in this area of research will be presented.

\section{A. Brief Historical Background of Leukotrienes and Their Receptors}

Ever since the identification of the biochemical structure of the leukotrienes and their association with inflammation (Samuelsson, 1983), the pathophysiological role of these lipid mediators has been explored in many experimental approaches (Brink et al., 2003). Initially, $\mathrm{LTB}_{4}$ was appreciated for its potent chemotactic effects on neutrophil granulocytes (Ford-Hutchinson et al., 1980; Malmsten et al., 1980; Palmblad et al., 1981) and its ability to serve as a complete secretagog for human peripheral blood neutrophils (Serhan et al., 1982). In contrast, initial studies of cysteinyl leukotrienes were mainly focused on their potent bronchoconstrictive effects (Dahlén et al., 1980). However, subsequent studies have extended these findings and associated leukotriene receptor signaling with several pathophysiological processes.

The first leukotriene receptor to be cloned was the human $\mathrm{BLT}_{1}$, and the molecular structure was reported in 1997 (Yokomizo et al., 1997). This molecular identification of the $\mathrm{BLT}_{1}$ receptor $\left(\mathrm{BLT}_{1} \mathrm{R}\right)$ permitted the characterization of functional $\mathrm{BLT}_{1} \mathrm{R}$ in several leukocyte populations (Kim and Luster, 2007) and also in nonmyeloid cells, such as vascular smooth muscle and endothelial cells (Bäck et al., 2005). Although the $\mathrm{LTB}_{4}$-induced interaction with $\mathrm{BLT}_{1} \mathrm{R}$ corresponded with several effects observed in those target cells, initial studies had revealed both high- and lower-affinity binding sites for $\mathrm{LTB}_{4}$ specifically in human granulocytes (Goldman and Goetzl, 1984). The molecular 
TABLE 1

Leukotriene receptors

For full details, see http://www.iuphar-db.org/DATABASE/ObjectDisplayForward?objectId=270\&familyId=35.

\begin{tabular}{|c|c|c|c|c|}
\hline Receptor & $\mathrm{BLT}_{1}$ & $\mathrm{BLT}_{2}$ & CysLT $_{1}$ & CysLT $_{2}$ \\
\hline \multicolumn{5}{|l|}{ Amino acids } \\
\hline$H s$ & 352 & 389 & 337 & 346 \\
\hline$M m$ & 351 & 360 & 339 & 309 \\
\hline$R n$ & 351 & 358 & 352 & 309 \\
\hline \multicolumn{5}{|l|}{ NCBI sequence no. } \\
\hline$H s$ & NP 858043 & NP 062813 & NP 006630 & NP 065110 \\
\hline $\mathrm{Mm}$ & NP_032545 & NP_065236 & NP_067451 & NP_598481 \\
\hline$R n$ & NP_067688 & NP_446092 & NP_446093 & NP_596904 \\
\hline \multicolumn{5}{|l|}{ Gene name } \\
\hline$H s$ & $L T B 4 R$ & LTB4R2 & CYSLTR1 & CYSLTR2 \\
\hline$M m$ & Ltb4r1 & $L t b 4 r 2$ & Cysltr1 & Cysltr2 \\
\hline$R n$ & $L t b 4 r$ & Ltb4r2 & Cysltr1 & Cysltr2 \\
\hline \multicolumn{5}{|l|}{ Chromosomal location } \\
\hline Hs & $14 q 11.2-q 12$ & $14 q 11.2-q 12$ & $\mathrm{Xq13.2-21.1}$ & $13 q 14.12-q 21.1$ \\
\hline$M m$ & $14 \mathrm{C} 2$ & $14 \mathrm{C} 1$ & $\mathrm{XD}$ & $14 \mathrm{D} 2$ \\
\hline$R n$ & $15 p 13$ & $15 p 13$ & $\mathrm{Xq} 31$ & $15 \mathrm{p} 11$ \\
\hline Selective ligands & $-^{a}$ & $-{ }_{a}^{1}$ & $-a$ & $-a$ \\
\hline Nonselective ligands & $\mathrm{LTB}_{4}$ & $\mathrm{LTB}_{4} ; 12-\mathrm{HHT}$ & $\mathrm{LTC}_{4} ; \mathrm{LTD}_{4} ; \mathrm{LTE}_{4}$ & $\mathrm{LTC}_{4} ; \mathrm{LTD}_{4} ; \mathrm{LTE}_{4}$ \\
\hline Selective antagonists & U75302; CP-105,696 & LY-255,283 & Montelukast; zafirlukast; pranlukast & CysLT2cpd \\
\hline Nonselective antagonists & ONO-4057 & ONO-4057 & BAY U9773 & BAY U9773 \\
\hline
\end{tabular}

Hs, Homo sapiens; Mm, Mus muscularis; Rn, Rattus norvegicus; ONO-4057, (5-[2-(2-carboxyethyl)-3-[6-(4-methoxyphenyl)-5E-hexenyl]oxyphenoxy]valeric acid.

${ }^{a}$ No ligands identified.

explanation for the latter finding was provided in 2000, when a gene with high sequence similarity to $\mathrm{BLT}_{1} \mathrm{R}$ was identified and encoded a low-affinity $\mathrm{LTB}_{4}$ receptor, which has subsequently been denoted $\mathrm{BLT}_{2} \mathrm{R}$ (Yokomizo et al., 2000; Brink et al., 2003). Transgenic overexpression of the human $\mathrm{BLT}_{1} \mathrm{R}$ in mice increased the inflammatory response to $\mathrm{LTB}_{4}$ (Chiang et al., 1999), whereas genetic $\mathrm{BLT}_{1} \mathrm{R}$ disruption decreased leukocyte chemotaxis and protected against disease development in response to several different proinflammatory stimuli (Haribabu et al., 2000; Tager et al., 2000; Brink et al., 2003) (see section II.E).

The classification of the CysLT receptors was initially based on the differential profiles of guinea pig trachea contractions induced by either $\mathrm{LTC}_{4}$ or $\mathrm{LTD}_{4}$ (Brink et al., 2003). Because a classification based on agonist selectivity is not generally applicable, the names $\mathrm{CysLT}_{1}$ and $\mathrm{CysLT}_{2}$ were introduced when referring to responses being sensitive and resistant (Labat et al., 1992) to the class of CysLT receptor antagonists, which had been developed for the treatment of asthma (Brink et al., 2003). The subsequent cloning of these receptors in 1999 and 2000 (Lynch et al., 1999; Sarau et al., 1999; Heise et al., 2000; Nothacker et al., 2000; Takasaki et al., 2000) largely confirmed these pharmacological characteristics of the $\mathrm{CysLT}_{1}$ and $\mathrm{CysLT}_{2}$ receptors.

\section{B. Chemical Structure of Endogenous Leukotriene Receptor Agonist}

Most endogenous ligands for the leukotriene receptors are derived from lipoxygenase metabolism of arachidonic acid and are shown in Fig. 1. The formation of leukotrienes by 5 -lipoxygenase (5-LO) involves the oxygenation at carbon number 5 of arachidonic acid to form 5-hydroperoxyeicosatetraenoic acid (HpETE). Subsequently, removal of hydrogen at carbon 10 from 5-HpETE leads to formation of the epoxide intermediate $\mathrm{LTA}_{4}$, which serves as precursor for LT synthesis (Shimizu et al., 1984; Rådmark and Samuelsson, 2009) (Fig. 1). Although detected in the cytosolic or nucleosolic fraction of resting cells, cellular activation leads to 5-LO translocation to the nuclear envelope in a calcium-dependent manner, where colocalization with the 5-LO-activating protein (FLAP) is a prerequisite for LT synthesis (Evans et al., 2008).

The enzyme LTA 4 hydrolase is located in the cytosol and stereospecifically adds water to carbon 12 of $\mathrm{LTA}_{4}$, leading to formation of $\mathrm{LTB}_{4}$ (Haeggström, 2004). Several inactivating pathways for $\mathrm{LTB}_{4}$ are known, of which $\omega$-oxidation has been most extensively studied in human leukocytes (for review, see Yokomizo et al., 2001b). In this pathway, $\mathrm{LTB}_{4}$ is converted to 20 -hydroxy-LTB 4 by $\mathrm{LTB}_{4} \omega$-hydroxylase and then to 20-carboxy- $\mathrm{LTB}_{4}$ as indicated in Fig. 1 (Yokomizo et al., 2001b). The latter metabolites may also act as ligands at the BLT receptors, which will be discussed below.

The other metabolic pathway of $\mathrm{LTA}_{4}$ involves the enzyme $\mathrm{LTC}_{4}$ synthase $\left(\mathrm{LTC}_{4} \mathrm{~S}\right)$, a microsomal glutathione transferase, which conjugates $\mathrm{LTA}_{4}$ with glutathione to form $\mathrm{LTC}_{4}$ (Fig. 1) (Austen, 2007). After transportation to the extracellular space by an ATP-dependent transporter (multidrug resistance-associated protein) that recognizes its glutathione moiety, $\mathrm{LTC}_{4}$ is metabolized by $\gamma$-glutamyl transpeptidase into $\mathrm{LTD}_{4}$. Subsequently, a serum dipeptidase, which cleaves the peptide bond between the cysteinyl and glycyl residues in the $\mathrm{LTD}_{4}$ side chain, leads to the formation of $\mathrm{LTE}_{4}$ (Fig. 1). Because $\mathrm{LTC}_{4}, \mathrm{D}_{4}$, and $\mathrm{E}_{4}$ all contain a cysteinyl group at carbon number 6 (Fig. 1), these LTs are referred to as the cysteinyl leukotrienes. Thus, the receptors for these LTs are termed CysLT receptors (Brink et al., 2003).

In addition to 5 - $\mathrm{LO}$ metabolites, products of the 12- and 15-lipoxygenase metabolism of arachidonic acid metabo- 

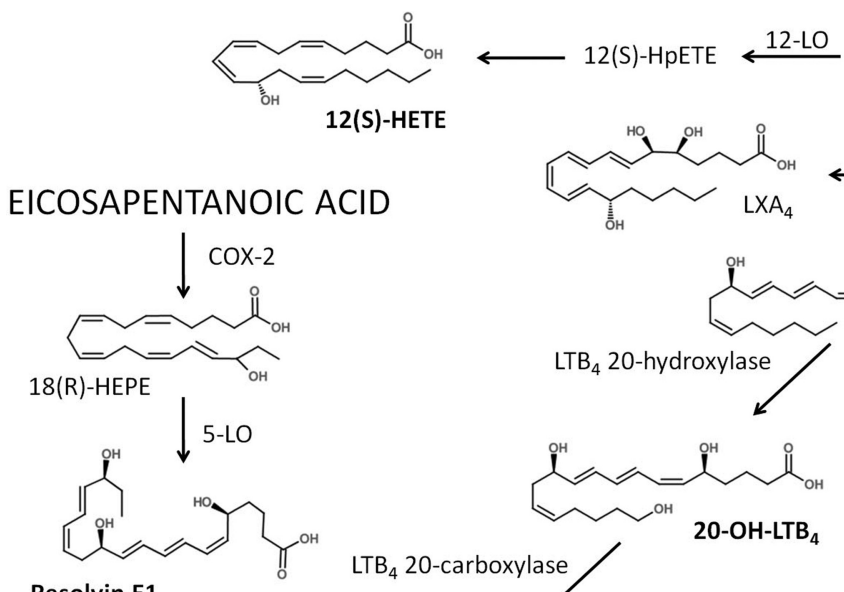

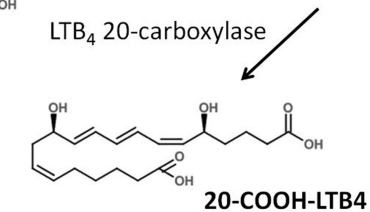
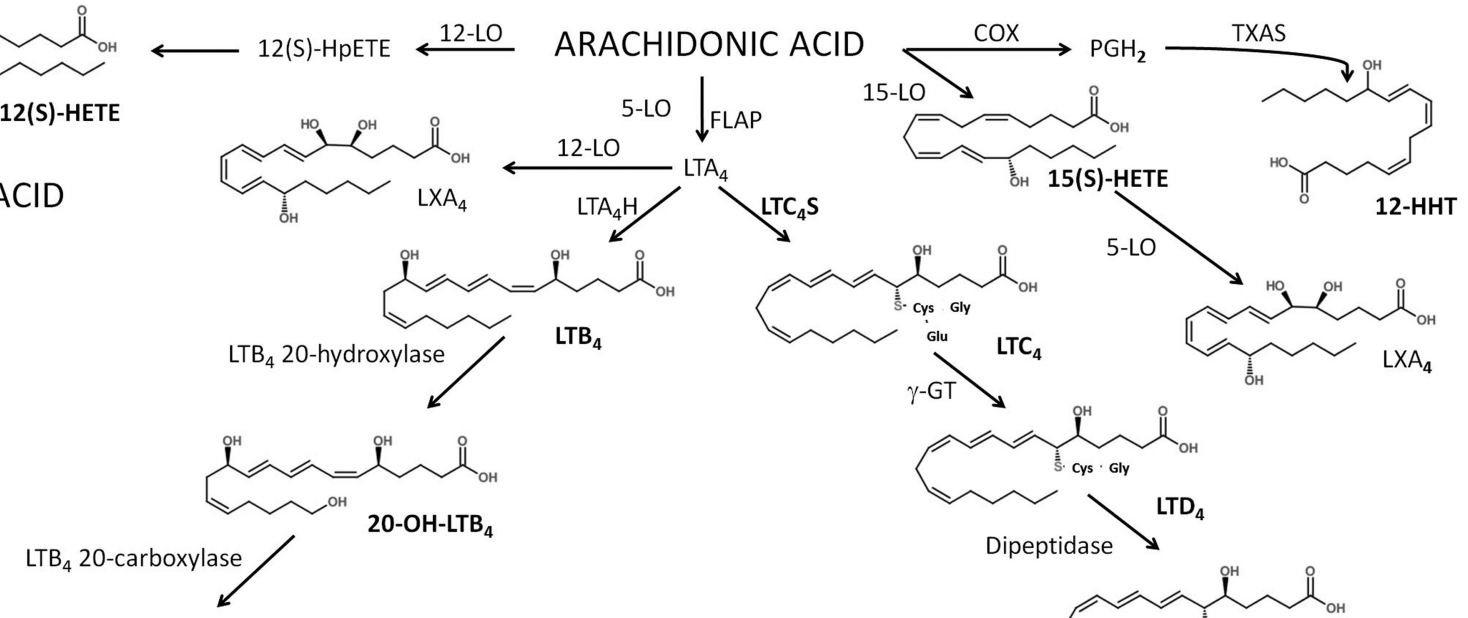

Resolvin E1

FIG. 1. Biosynthesis and chemical structures of endogenous leukotriene receptor agonists, derived from arachidonic acid. Lipoxin (LX) biosynthesis pathways in humans via 15-LO and 5-LO interaction or 12-LO via 5-LO derived $\mathrm{LTA}_{4}$ are also depicted. In addition, resolvin E1, derived from eicosapentaenoic acid (EPA), may act as a partial $\mathrm{BLT}_{1} \mathrm{R}$ agonist. GT, glutamyl transferase; HEPE, hydroxyeicosapentaenoic acid; $\mathrm{LTA}_{4} \mathrm{H}$, $\mathrm{LTA}_{4}$ hydrolase; TXAS, thromboxane synthase.

lism can act as ligands for the LT receptors (Fig. 1). For example, 12(R)-hydroxyeicosatetraenoic acid (HETE) competes with $\mathrm{LTB}_{4}$ binding at $\mathrm{BLT}_{1} \mathrm{R}$ (Yokomizo et al., 1997, 2001c), whereas 12(S)-HETE, $12(S)$-HpETE, and 15(S)HETE activate $B_{2} \mathrm{R}$ (Yokomizo et al., 2001c). The orphan receptor GPR31 has been suggested as a high-affinity receptor for 12(S)-HETE (Guo et al., 2011). Finally, 12-HHT (Fig. 1), which is formed through the cyclooxygenase pathway of arachidonic acid metabolism is a highaffinity $\mathrm{BLT}_{2} \mathrm{R}$ agonist (Okuno et al., 2008).

\section{BLT Receptors}

\section{A. BLT Receptor Subtypes}

1. $B L T_{1}$ Receptor. The open reading frame of the human $L T B 4 R$ gene encodes the $B_{1} T_{1}$. The $B T_{1} R$ protein consists of 352 aa (NCBI Reference Sequence: NP_858043) (Fig. 2). The BLT ${ }_{1} \mathrm{R}$ has also been isolated in other species, with a homology similar to that of the human protein in mice (78\%; Huang et al., 1998), in guinea pigs (78\%; Masuda et al., 1999), and in rats (80\%; Toda et al., 1999).

Although $\mathrm{LTB}_{4}$ seems to be the sole full agonist at $\mathrm{BLT}_{1} \mathrm{R}$, binding studies in membrane fractions of $\mathrm{BLT}_{1} \mathrm{R}$-transfected cells have shown significant competitions for 20-OH-LTB 4 , 12-oxo-LTB 4 and 12-epi-LTB 4 , $20-\mathrm{COOH}-\mathrm{LTB}_{4}$, and $12(R)$-HETE (Yokomizo et al., 1997, 2001c). However, not all of these ligands seem to induce similar intracellular signaling. For example, studies of the recombinant guinea pig $\mathrm{BLT}_{1} \mathrm{R}$ have shown that although $20-\mathrm{OH}-\mathrm{LTB}_{4}$ was less potent than $\mathrm{LTB}_{4}$ in the mobilization of intracellular $\mathrm{Ca}^{2+}\left(\left[\mathrm{Ca}^{2+}\right]_{\mathrm{i}}\right)$,

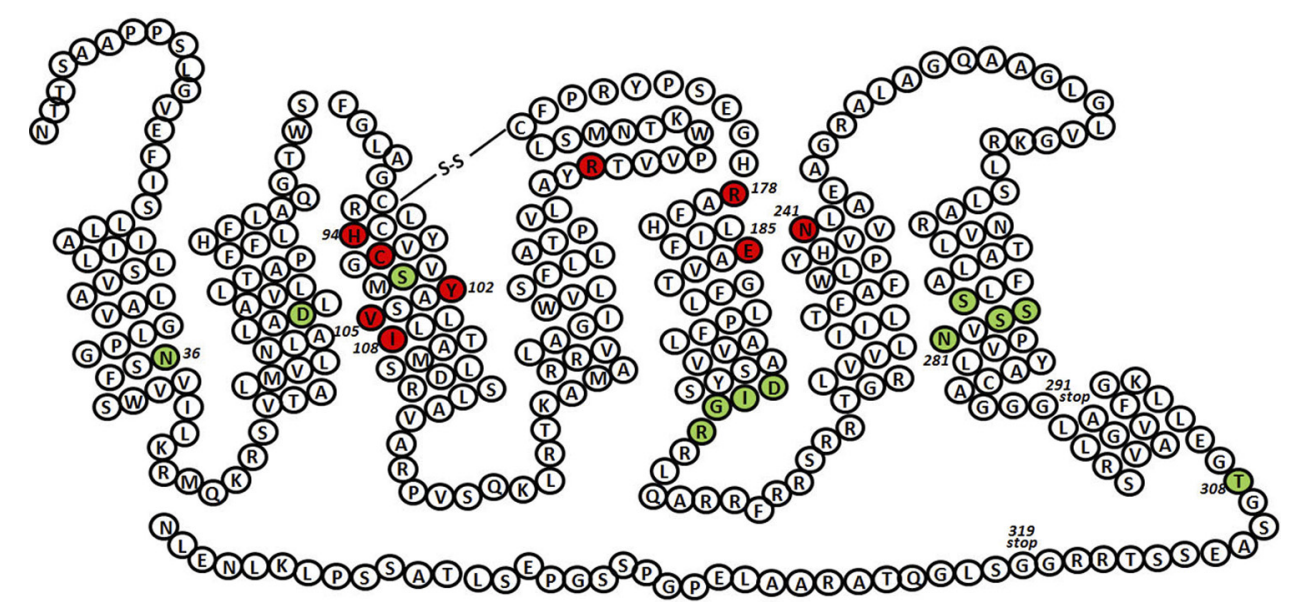

FIG. 2. Schematic representation of the human BLT receptor with the boundaries of each transmembrane, extracellular, and intracellular domain derived from the molecular modeled structure described previously (Basu et al., 2007; Kuniyeda et al., 2007). The amino acid residues involved in the ligand binding are shown in red, whereas residues implicated in receptor signaling are shown in green. The C-tail truncations in the described mutants (see text) are indicated by "stop". 
these agonists were equally potent in inhibiting cAMP formation (Masuda et al., 1999). In addition, the eicosapentaenoic acid metabolite resolvin (Rv) E1 was identified as a partial $\mathrm{BLT}_{1} \mathrm{R}$ agonist. In particular, RvE1 selectively inhibited adenylate cyclase and attenuated $\mathrm{LTB}_{4}$-induced nuclear factor $\kappa \mathrm{B}(\mathrm{NF}-\kappa \mathrm{B})$ activation in $\mathrm{BLT}_{1}$ - but not in $\mathrm{BLT}_{2}$-transfected cells, whereas in human peripheral blood mononuclear cells RvE1 partially induced calcium mobilization and blocked subsequent stimulation by $\mathrm{LTB}_{4}$ (Arita et al., 2007). These data thus suggest that this mediator, besides activating the receptor ChemR23, could act through inhibition of $\mathrm{LTB}_{4}$-induced signaling. More information on this new genus of specialized proresolving mediators has been published recently (Bannenberg and Serhan, 2010).

Initially, $\mathrm{BLT}_{1} \mathrm{R}$ expression was reported to be restricted to phagocytic leukocytes (Yokomizo et al., 1997), and subsequent studies identified $\mathrm{BLT}_{1} \mathrm{R}$ expression in granulocytes, monocytes, and dendritic cells. In addition, lymphocytes also express $\mathrm{BLT}_{1} \mathrm{R}$ (see section II.D), and functional $\mathrm{BLT}_{1} \mathrm{R}$ are now known to be expressed in nonmyeloid cells, such as vascular smooth muscle cells (SMCs) (Bäck et al., 2005), as well as endothelial cells (ECs) (Qiu et al., 2006), skeletal muscle satellite cells (Sun et al., 2009), and neural stem cells (Wada et al., 2006).

2. $B L T_{2}$ Receptor. In the analysis of human and mouse $L T B 4 R$ genes, an open reading frame encoding a putative seven-transmembrane-type receptor with sequence similarities to $\mathrm{BLT}_{1} \mathrm{R}$ was identified (Yokomizo et al., 2000). This gene was shown to encode a receptor protein that exhibited $\mathrm{LTB}_{4}$ binding with a 20 -fold higher $K_{\mathrm{d}}$, and calcium signaling with a 30 -fold higher $\mathrm{EC}_{50}$ value compared with $\mathrm{BLT}_{1} \mathrm{R}$ (Yokomizo et al., 2000). The gene has been designated LTB4R2, and the receptor has been classified as the $\mathrm{BLT}_{2}$ receptor $\left(\mathrm{BLT}_{2} \mathrm{R}\right)$ (Brink et al., 2003). The $\mathrm{BLT}_{2} \mathrm{R}$ protein consists of 358 aa (NCBI Reference Sequence: NP_062813) and exhibits a 36 to $45 \%$ aa identity with the human BLT $_{1} \mathrm{R}$ (Kamohara et al., 2000; Tryselius et al., 2000; Yokomizo et al., 2000). The murine $\mathrm{BLT}_{2} \mathrm{R}$ has a $92 \%$ aa homology with the human receptor protein, which is a higher homology compared with that of $\mathrm{BLT}_{1} \mathrm{R}$ ( $78 \%$ aa identity between murine and human proteins; Iizuka et al., 2005). These data suggest that the $B_{2 T} R$ has been conserved during evolution.

In contrast to the relatively specific binding of $\mathrm{LTB}_{4}$ at $\mathrm{BLT}_{1} \mathrm{R}$, several lipoxygenase products in addition to $\mathrm{LTB}_{4}$ have been identified as ligands for $\mathrm{BLT}_{2} \mathrm{R}$. These include 12(S)-HETE, $12(S)$-HpETE, and 15(S)-HETE (Fig. 1) (Yokomizo et al., 2001c). Furthermore, the thromboxane synthase product 12 -HHT formed in activated blood platelets and macrophages from prostaglan$\operatorname{din} \mathrm{H}_{2}$ (Fig. 1) is also a natural ligand for $\mathrm{BLT}_{2} \mathrm{R}$ (Okuno et al., 2008). In the latter study, the $\mathrm{EC}_{50}$ values of 12-HHT and $\mathrm{LTB}_{4}$ for $\left[\mathrm{Ca}^{2+}\right]_{\mathrm{i}}$ mobilization in CHO$\mathrm{BLT}_{2} \mathrm{R}$ cells were 19 and $142 \mathrm{nM}$, respectively, whereas
12-HHT failed to induce calcium mobilization in CHO$\mathrm{BLT}_{1} \mathrm{R}$ cells (Okuno et al., 2008). Several binding studies further demonstrated that $\mathrm{BLT}_{2} \mathrm{R}$ is a high-affinity receptor for $12-\mathrm{HHT}$ and that $12-\mathrm{HHT}$ and $\mathrm{LTB}_{4}$ occupy the same binding site on $\mathrm{BLT}_{2} \mathrm{R}$ (Okuno et al., 2008). A synthetic selective $\mathrm{BLT}_{2} \mathrm{R}$ agonist has also been reported and termed compound A (Iizuka et al., 2005).

In $\mathrm{CHO}$ cells expressing the human $\mathrm{BLT}_{2} \mathrm{R}$, maximal chemotaxis was observed at $\mathrm{LTB}_{4}$ concentrations greater than $1 \mu \mathrm{M}$, compared with $10 \mathrm{nM}$ for $\mathrm{CHO}$ cells expressing human BLT $_{1} \mathrm{R}$ (Yokomizo et al., 2001a; Okuno et al., 2008). Data suggest that coexpression of both human $\mathrm{BLT}_{1}$ and $\mathrm{BLT}_{2}$ receptors makes CHO cells migrate toward both very low and high $\mathrm{LTB}_{4}$ concentrations (Yokomizo et al., 2001a). Other hydroxyeicosanoids acting as $\mathrm{BLT}_{2} \mathrm{R}$ agonists, such as $12(S)$-HETE, 12(R)HETE, and 12-epi-LTB ${ }_{4}$, require even higher concentrations (approximately $10 \mu \mathrm{M}$ ) to induce a maximal chemotactic response (Yokomizo et al., 2001c). In contrast, maximal chemotaxis for $12-\mathrm{HHT}$ in $\mathrm{CHO}$ cells expressing the human $\mathrm{BLT}_{2} \mathrm{R}$ is observed at $30 \mathrm{nM}$, suggesting that this high-affinity $\mathrm{BLT}_{2} \mathrm{R}$ ligand is coupled to chemotaxis (Okuno et al., 2008). In line with findings in $\mathrm{CHO}$ cells, murine $\mathrm{BLT}_{2} \mathrm{R}$-expressing 300.19 cells exhibit higher affinity for 12 -HHT than for $\mathrm{LTB}_{4}$. 12-HHTinduced intracellular calcium flux with the same efficacy as $\mathrm{LTB}_{4}$ (Mathis et al., 2010). However, in the latter cells, 12-HHT was only a weak agonist for chemotaxis with 3\% activity relative to $\mathrm{LTB}_{4}$ (Mathis et al., 2010), suggesting a ligand-specific coupling to chemotaxis. Studies of primary cells have generated variable results as to the role of $\mathrm{BLT}_{2} \mathrm{R}$ in $\mathrm{LTB}_{4}$-induced chemotaxis (see section II.D).

The most abundant $\mathrm{BLT}_{2} \mathrm{R}$ expression is found in the spleen, followed by liver, ovary, and leukocytes, and $\mathrm{BLT}_{2} \mathrm{R}$ expression is also detected in several other organs, suggesting a ubiquitous expression pattern, at least in human tissues (Yokomizo et al., 2000). The tissue distribution of $\mathrm{BLT}_{2} \mathrm{R}$ is different in mouse tissues, with the highest expression in small intestine followed by colon and skin (Iizuka et al., 2005). Physiological and pathophysiological responses associated with $\mathrm{BLT}_{2} \mathrm{R}$ signaling include macrophage and mast cell (MC) chemotaxis (Subbarao et al., 2004; Lundeen et al., 2006; Okuno et al., 2008), guinea pig lung parenchyma contraction (Sakata et al., 2004), and mouse models of angiogenesis (Kim et al., 2009), itch-associated scratching (Kim et al., 2008), colitis (Iizuka et al., 2010), and arthritis (Mathis et al., 2010).

3. Genomic Organization and Transcriptional Regulation. The genes encoding $\mathrm{BLT}_{1} \mathrm{R}$ and $\mathrm{BLT}_{2} \mathrm{R}$ are designated $L T B 4 R$ and $L T B 4 R 2$, respectively (Table 1), and are located within 10 kilobase pairs of each other in both the human and murine genome (Yokomizo et al., 2000). The genes encoding the BLT receptors form a gene cluster on human chromosome 14q11.2-q12 with the open reading frame of the $L T B 4 R 2$ gene overlapping one of 
the promoter regions of the human LTB4R gene (Kato et al., 2000; Yokomizo et al., 2000; Brink et al., 2003). The $L T B 4 R$ gene consists of three exons, and, in line with other receptors for chemoattractants such as fMet-LeuPhe and interleukin (IL)-8, the open reading frame is intronless (Kato et al., 2000). One of the LTB4R promoters has been shown to contain consensus sequence for $\mathrm{NF}-\kappa \mathrm{B}$ and Sp1 binding (Kato et al., 2000). Spontaneous mutations around the LTB4R and LTB4R2 genes have been reported to be associated with cerebrovascular disease (Bevan et al., 2008), but the close proximity of the genes encoding those receptors suggests that those polymorphisms cannot be ascribed to either of the genes alone.

In THP-1 cells, the deletion of a Sp1 binding site in the $L T B 4 R$ promoter decreases transcriptional activity (Kato et al., 2000). In human monocytes, $B_{1} T_{1} R$ mRNA expression is down-regulated by proinflammatory stimuli, such as lipopolysaccharide (LPS), interferon (IFN)- $\gamma$, and tumor necrosis factor (TNF)- $\alpha$, whereas the antiinflammatory cytokine IL-10 and dexamethasone upregulate $B_{1} T_{1} R$ mRNA in these cells (Pettersson et al., 2005). Likewise, in human neutrophil granulocytes, $\mathrm{BLT}_{1} \mathrm{R}$ mRNA is up-regulated by dexamethasone (Stankova et al., 2002), and $\mathrm{BLT}_{1} \mathrm{R}$ protein and $\mathrm{LTB}_{4}$ binding are decreased by TNF- $\alpha$ and LPS (O'Flaherty et al., 1991; Pettersson et al., 2003). The latter findings support earlier studies demonstrating decreased $\mathrm{LTB}_{4}$ binding and $\mathrm{LTB}_{4}$-induced chemotaxis in neutrophils derived from endotoxemic rabbits (Hartiala et al., 1985; Goldman et al., 1986). Of interest, the transcriptional regulation of the $L T B 4 R$ gene may vary between cells of hematopoietic and nonhematopoietic origin (Kato et al., $2000)$. In contrast to findings in leukocytes, $B_{L} T_{1} R$ expression is up-regulated by LPS, IL- $1 \beta$, and IFN- $\gamma$ in vascular SMCs, as demonstrated by studies of cells derived from human, rat, or mouse vessels (Bäck et al., 2005; Heller et al., 2005). Furthermore, vascular injury in vivo significantly up-regulates $B T_{1} R$ mRNA through $\mathrm{NF}-\kappa \mathrm{B}$ signaling (Bäck et al., 2005). Likewise, whereas $\mathrm{BLT}_{1} \mathrm{R}$ is expressed at low levels in human umbilical cord endothelial cells (HUVECs) under resting conditions, either LPS or IL- $1 \beta$ induces BLT $_{1} \mathrm{R}$ mRNA and protein levels and renders HUVECs responsive to $\mathrm{LTB}_{4}$ (Qiu et al., 2006). This cell-specific expression of $\mathrm{BLT}_{1} \mathrm{R}$ may be dependent on epigenetic regulation, for example, through methylation of the promoter region, which has been shown to regulate $B T_{1} R$ transcription in vitro (Kato et al., 2000). In a recent study, the enhancer in the human $L T B 4 R$, termed $A E$-BLex, was discovered at the intron I-exon-II boundary (Hashidate et al., 2010). $A E$ $B L e x$ possesses two acute myeloid leukemia 1 (AML1, also known as Runx1) recognition sites. The AML1/AE$B$ Lex complex was confirmed in several $\mathrm{BLT}_{1} \mathrm{R}$-expressing leukemia cell lines and human peripheral leukocytes. Thus, AML1 enhances $B_{1} T_{1} R$ expression by binding to AE-BLex, which is accessible in leukocytes.
The enhancement of the $\mathrm{BLT}_{1} \mathrm{R}$ expression in leukocytes is due to a loosening of the chromatin structure around AE-BLex, leading to the incremental binding of AML1 (Hashidate et al., 2010).

\section{B. Structure-Function Relationships for BLT Receptors}

$\mathrm{LTB}_{4}$ binds near the extracellular surface of $\mathrm{BLT}_{1} \mathrm{R}$, and although polar residues within transmembrane domains (TMs) III, V, and VI and extracellular loop 2 are critical for ligand binding, polar residues in TMs II, III, and VII play a central role in transducing the ligandinduced conformational change to activation (Sabirsh et al., 2006; Basu et al., 2007) (Fig. 2). Furthermore, a conformational change of TM IV takes place during ligand binding (Baneres et al., 2003).

Site-directed mutagenesis has revealed several of the residues involved in $\mathrm{LTB}_{4}$ binding to $\mathrm{BLT}_{1} \mathrm{R}$ (Fig. 2). Although initial studies had suggested that mutating all receptor cysteines (except $\mathrm{Cys}^{90}$ and $\mathrm{Cys}^{168}$ ) to serine did not alter ligand binding, selective replacement of $\mathrm{Cys}^{97}$ in the TM III domain to serine leads to a 100 -fold decrease in the affinity for the agonist (Mesnier and Banères, 2004). Furthermore, residues $\mathrm{His}^{94}$, $\mathrm{Tyr}^{102}$, $\mathrm{Val}^{105}$, and Ile ${ }^{108}$ in TM III, Asn ${ }^{241}$ in TM IV, and $\mathrm{Arg}^{178}$ and Glu ${ }^{185}$ in TM IV have been shown to be necessary for ligand binding (Sabirsh et al., 2006; Basu et al., 2007) (Fig. 2). In addition to the TM domains, the second extracellular loop of $\mathrm{BLT}_{1} \mathrm{R}$ may play an even more critical role for $\mathrm{LTB}_{4}$ binding as suggested by the complete loss of ligand binding after mutation of $\mathrm{Arg}^{156}$ to alanine (Basu et al., 2007) (Fig. 2). In line with the homology between the $\mathrm{BLT}_{1}$ and $\mathrm{BLT}_{2}$ receptors (see section II.A), most of the binding site residues described above suggest a common binding mode for $\mathrm{LTB}_{4}$ at the two BLT receptor subtypes (Basu et al., 2007). However, the presence of $\mathrm{Tyr}^{94}$ in $\mathrm{BLT}_{2} \mathrm{R}$ at the place of $\mathrm{His}^{94}$ within $\mathrm{BLT}_{1} \mathrm{R}$ has been suggested to account for some of the differences in the binding affinities and agonist selectivity between these receptors (Basu et al., 2007).

Structural models for the ligand-free and ligandbound states of $B_{1} T_{1} R$ have revealed an activation core formed around $\mathrm{Asp}^{64}$, and mutagenesis toward $\mathrm{Asn}^{36}$, $\mathrm{Ser}^{100}$, and $\mathrm{Asn}^{281}$ and a triad of serines, $\operatorname{Ser}^{277}, \mathrm{Ser}^{278}$ and $\operatorname{Ser}^{279}$ (Fig. 2), resulted in loss of signaling capacity, whereas mutant receptors retained normal $\mathrm{LTB}_{4}$ binding (Basu et al., 2007). Furthermore, mutating the third intracellular loop, which consists of the cytoplasmic end of TM V (Fig. 2), reduced $\mathrm{G}_{\mathrm{i}}$-dependent signaling associated with a loss of the high-affinity $\mathrm{LTB}_{4}$ binding state (Kuniyeda et al., 2007).

The C-tail containing a putative cytoplasmic helical domain, helix 8, of the BLT receptors has recently received attention. For example, $\mathrm{BLT}_{1} \mathrm{R}$ with a mutated helix 8 exhibit increased $\mathrm{LTB}_{4}$ binding as well as prolonged $\left[\mathrm{Ca}^{2+}\right]_{\mathrm{i}}$ mobilization and cellular metabolic activation compared with the WT receptor, suggesting that helix 8 might work as a scaffold for $G$ proteins, changing 
$\mathrm{BLT}_{1} \mathrm{R}$ to a low-affinity state leading to release of the ligand and signal shutdown (Okuno et al., 2003). In contrast, helix 8 may not be critical for either ligand binding or activation of $\mathrm{BLT}_{2} \mathrm{R}$ (Yasuda et al., 2009). However, human $\mathrm{BLT}_{2} \mathrm{Rs}_{\mathrm{s}}$ lacking helix 8 accumulate in the endoplasmic reticulum, suggesting that helix 8 may be required for $\mathrm{BLT}_{2} \mathrm{R}$ protein folding and passage through the endoplasmic reticulum to the cell membrane (Yasuda et al., 2009).

Whereas cells expressing a complete C-tail-truncated $\mathrm{BLT}_{1} \mathrm{R}$ (G291stop, Fig. 2) exhibit increased numbers of binding sites and increased signal transduction compared with wild-type $\mathrm{BLT}_{1} \mathrm{R}$ (Gaudreau et al., 2004), a partially C-tail-truncated $\mathrm{BLT}_{1} \mathrm{R}$ mutant (G319stop, Fig. 2) has shown binding characteristics similar to WT $\mathrm{BLT}_{1} \mathrm{R}$ (Gaudreau et al., 2002). Furthermore, mutating a dileucine motif (Leu ${ }^{304}-\mathrm{Leu}^{305}$ ), suggested to be involved in a hydrophobic core (Fig. 2), mimicked the increased binding and signaling associated with the complete C-tail truncated receptor (Gaudreau et al., 2004). In addition, this latter mutation prevented $\mathrm{LTB}_{4}$-induced $\mathrm{BLT}_{1} \mathrm{R}$ internalization (Gaudreau et al., 2004), which corroborated the findings that the complete $\mathrm{C}$ tail-truncated $\mathrm{BLT}_{1} \mathrm{R}$ lost the capacity to internalize through the involvement of GPCR kinases (GRK) 2 and 6 (Gaudreau et al., 2002; Chen et al., 2004). In addition, substitution of $\mathrm{Thr}^{308}$ within a putative casein kinase 2 site in the full-length $\mathrm{BLT}_{1} \mathrm{R}$ (Fig. 2) prevented most of GRK6-mediated inhibition of $\mathrm{LTB}_{4}$-induced inositol phosphate production while only partially affecting the $\mathrm{LTB}_{4}$-induced $\mathrm{BLT}_{1} \mathrm{R}$ phosphorylation (Gaudreau et al., 2002). Through the substitution of all Ser and Thr residues in the C-terminal tail with Ala (to generate a phosphorylation-defective mutant) or with Asp/Glu (to mimic constitutive phosphorylation), it has been shown that $\mathrm{BLT}_{1} \mathrm{R}$ phosphorylation may be an important mediator of $\mathrm{G}$ protein activation, whereas $\beta$-arrestin-associated $\mathrm{BLT}_{1} \mathrm{R}$ internalization seems to be independent of phosphorylation (Jala et al., 2005). For the murine $\mathrm{BLT}_{1} \mathrm{R}$, a PKC consensus phosphorylation site has been reported to be located on the second intracellular loop, and a $\operatorname{Ser}^{127}$ substitution within this site prevented $\mathrm{mBLT}_{1} \mathrm{R}$ desensitization (Mollerup et al., 2007).

\section{Intracellular Signaling Pathways and Second-Messenger Systems}

The downstream intracellular signaling pathways after $\mathrm{BLT}_{1}$ and $\mathrm{BLT}_{2}$ receptor activation involve increased $\left[\mathrm{Ca}^{2+}\right]_{\mathrm{i}}$ and inhibition of adenylyl cyclase (Brink et al., 2003). The G protein coupling for the BLT receptors may depend on the type of $\mathrm{G}$ proteins expressed in the different cells studied. In several cell types expressing endogenous BLT receptors, pertussis toxin (PTX) inhibits the increased $\left[\mathrm{Ca}^{2+}\right]_{\mathrm{i}}$ in response to $\mathrm{LTB}_{4}$, suggesting coupling to $\mathrm{G}_{\mathrm{i}}$ proteins (Brink et al., 2003). However, in $\mathrm{BLT}_{1} \mathrm{R}$-transfected cells, a significant portion of the $\mathrm{LTB}_{4}$-induced $\left[\mathrm{Ca}^{2+}\right]_{\mathrm{i}}$ response is resistant to PTX, sug- gesting also the involvement of $\mathrm{G}_{\mathrm{q}}$-protein coupling (Yokomizo et al., 1997; Haribabu et al., 1999; Brink et al., 2003; Sabirsh et al., 2004). These different profiles of PTX sensitivity suggest that $B L T_{1} R$ and $B L T_{2} R$ signal through different subtypes of $G_{i}$ proteins (Yokomizo et al., 2000).

BLT receptor transduction also activates a number of kinases that phosphorylate downstream signal transduction proteins (Brink et al., 2003). For example, phosphorylation of mitogen-activated protein kinases (MAPKs) is involved in the $\mathrm{LTB}_{4}$-induced proliferation of RAW 264.7 macrophages and bronchial SMCs (Nieves and Moreno, 2006; Watanabe et al., 2009). In addition, extracellular signal-regulated kinases (ERKs) may also be involved in $\mathrm{LTB}_{4}$-induced proliferation, as demonstrated in vascular SMCs (Heller et al., 2005) and in signaling associated with delayed neutrophil apoptosis (Pétrin et al., 2006). The reported effects of different kinase inhibitors may also depend on which $\mathrm{LTB}_{4}$-induced response is studied. For example, wortmannin, a phosphatidylinositol 3-kinase (PI3K) inhibitor has been reported to block $\mathrm{LTB}_{4}$-induced chemotaxis, whereas calcium mobilization remains intact (Haribabu et al., 1999; Sabirsh et al., 2004). Tyrosine kinases have been implicated in $\mathrm{LTB}_{4}$-signaling in neutrophil granulocytes (Dryden et al., 1992) and BLT $_{1}$-transfected HeLa cells (Sabirsh et al., 2004).

Effects on transcription have been implicated in BLT receptor intracellular signaling. For example, treatment of monocytes with $\mathrm{LTB}_{4}$ increased the transcriptional activation of the IL- 6 gene (Brach et al., 1992). In this study, a reporter gene assay identified two restricted regions within the IL-6 promoter. These binding sites for NF-IL-6 and NF- $\kappa$ B conferred inducibility by $\mathrm{LTB}_{4}$. Exogenous addition of $\mathrm{LTB}_{4}$ increased the DNA binding of these transcription factors in monocytes (Brach et al., 1992; Huang et al., 2004) and induced nuclear translocation of NF- $\kappa \mathrm{B}$ p65 in murine bone marrow-derived dendritic cells (Toda et al., 2010). The $\mathrm{LTB}_{4}$-induced NF $-\kappa \mathrm{B}$ DNA binding activity was abolished by the $\mathrm{BLT}_{1} \mathrm{R}$ antagonist 1-[3-(4-phenyl-benzyl)-4-hydroxy-chroman-7-yl] cyclopentane carboxylic acid (CP105,696) (Huang et al., 2004), suggesting that transcriptional alterations by $\mathrm{LTB}_{4}$ may be through $\mathrm{BLT}_{1} \mathrm{R}$. One possible mechanism for this transcriptional activation by $\mathrm{LTB}_{4}$ could be through $\mathrm{LTB}_{4}$-induced intracellular $\mathrm{H}_{2} \mathrm{O}_{2}$ formation (Gagnon et al., 1989), because the oxidant scavenger $N$-acetyl-L-cysteine completely blocks $\mathrm{LTB}_{4}$-mediated transcription factor binding in monocytes (Brach et al., 1992). However, another study failed to observe any effects of other antioxidants on IL-6 protein secretion (Rola-Pleszczynski and Stanková, 1992). In the latter study, the partial inhibition of $\mathrm{LTB}_{4}$-induced IL- 6 secretion by genistein suggested the involvement of tyrosine phosphorylation events during this process. 


\section{Receptor Distribution and Cellular Targets}

1. Neutrophil Granulocytes. The expression of both $\mathrm{BLT}_{1}$ and $\mathrm{BLT}_{2}$ receptor proteins in neutrophil granulocytes has, for example, been demonstrated by immunohistochemical analysis of surgical specimens derived from human abdominal aortic aneurysms (Houard et al., 2009). The potent neutrophil chemotactic activity of $\mathrm{LTB}_{4}$ through $\mathrm{BLT}_{1} \mathrm{R}$ signaling has been well established (Ford-Hutchinson et al., 1980; Malmsten et al., 1980; Palmblad et al., 1981). In neutrophils derived from the synovial fluid of patients with rheumatoid arthritis, $\mathrm{BLT}_{1}$ receptor antagonism inhibits $\mathrm{LTB}_{4}$-induced calcium mobilization and produces a rightward shift of the peak chemotactic response. In contrast, the $\mathrm{BLT}_{2} \mathrm{R}$ antagonist 1-[5-ethyl-2-hydroxy-4-[[6-methyl-6-(1H-tetrazol-5-yl) heptyl]oxy]phenyl]ethanone (LY255283) only inhibits $\left[\mathrm{Ca}^{2+}\right]_{\mathrm{i}}$ and the chemotactic response to $\mathrm{LTB}_{4}$ at high concentrations, at which this compound may also act as an antagonist at $\mathrm{BLT}_{1} \mathrm{R}$ (Mathis et al., 2010). Studies in knockout mouse models have also shown that the $\mathrm{LTB}_{4}$ induced chemotaxis and calcium mobilization in zymosanelicited murine peritoneal neutrophils derived from either WT or $\mathrm{BLT}_{2}$ knockout mice display similar $\mathrm{LTB}_{4}$ doseresponse relations, whereas neutrophils from $B_{1} T_{1} R$ knockout and $\mathrm{BLT}_{1} \mathrm{R}-\mathrm{BLT}_{2} \mathrm{R}$ knockout mice lack both chemotaxis and alterations of $\left[\mathrm{Ca}^{2+}\right]_{i}$ in response to $\mathrm{LTB}_{4}$ (Haribabu et al., 2000; Tager et al., 2000; Terawaki et al., 2005; Mathis et al., 2010).

In addition to chemotaxis, $\mathrm{LTB}_{4}$ stimulates neutrophil release of lysozyme (Hafstrom et al., 1981), matrix metalloproteinases (MMP) (Kjeldsen et al., 1992), myeloperoxidase (Terawaki et al., 2005), elastase, $\alpha$-defensins (Flamand et al., 2007), and azurocidin (Di Gennaro et al., 2009). Initial characterization of the binding of $\left[{ }^{3} \mathrm{H}\right] \mathrm{LTB}_{4}$ in relation to the functional effects of $\mathrm{LTB}_{4}$ on human neutrophils suggested that whereas high-affinity binding sites mediated chemotaxis, this lysosomal degranulation occurred by $\mathrm{LTB}_{4}$ binding to low-affinity sites (Goldman and Goetzl, 1984). Subsequent results demonstrated that both subsets of $\mathrm{LTB}_{4}$ binding sites contributed to increases in $\left[\mathrm{Ca}^{2+}\right]_{\mathrm{i}}$ induced by $\mathrm{LTB}_{4}$ (Goldman et al., 1985). In line with those findings, the neutrophil secretagog activity of $\mathrm{LTB}_{4}$ has consistently been observed at higher $\mathrm{LTB}_{4}$ concentrations compared with those needed to induce chemotaxis (Serhan et al., 1982). For example, maximal release of $\alpha$-defensins from neutrophils was observed at $100 \mathrm{nM} \mathrm{LTB}_{4}$ (Flamand et al., 2007), and the release of azurocidin was gradually increased at $\mathrm{LTB}_{4}$ concentrations ranging from $10 \mathrm{nM}$ to $1 \mu \mathrm{M}$ (Di Gennaro et al., 2009). The latter apparent low-affinity response, however, was inhibited by the selective $B T_{1} R$ antagonist CP-105,696, but not by the selective $\mathrm{BLT}_{2} \mathrm{R}$ antagonist LY255238. Despite the lowaffinity profile, further support for lysosomal enzyme release being mediated through $B_{L} T_{1} R$ rather than $\mathrm{BLT}_{2} \mathrm{R}$ signaling has emerged from studies of knockout mice. Although a low-affinity binding site for $\mathrm{LTB}_{4}$ has been characterized in neutrophils derived from $\mathrm{BLT}_{1} \mathrm{R}$ knockout mice, the myeloperoxidase release in response to $\mathrm{LTB}_{4}$ is also abolished in those cells (Terawaki et al., 2005), hence challenging the concept of lysosomal enzyme release through $\mathrm{BLT}_{2} \mathrm{R}$ signaling.

In addition to being a major neutrophil chemoattractant and promoting the release of lysosomal enzymes (Hafstrom et al., 1981; Serhan et al., 1982), prolonged exposure of neutrophils to $\mathrm{LTB}_{4}$ delays their constitutive apoptosis in a time- and concentration-dependent manner (Hébert et al., 1996). Furthermore, threshold levels of $\mathrm{LTB}_{4}(0.01 \mathrm{nM})$ induce surface expression of adhesion molecules, such as the $\beta 2$-integrin CD11b and Mac-1, which have been implicated in $\mathrm{LTB}_{4}$-induced neutrophil adhesion to endothelial cells (Gimbrone et al., 1984; Showell et al., 1998). The antimicrobial neutrophil response may also be facilitated by $\mathrm{LTB}_{4}$ through increased surface expression of Toll-like receptors 7, 8, and 9 (Gaudreault and Gosselin, 2009) and stimulated secretion of the cathelicidin LL-37 (Flamand et al., 2007; Wan et al., 2007).

2. Eosinophil Granulocytes. Western blot analysis has demonstrated $\mathrm{BLT}_{1} \mathrm{R}$ expression in eosinophils of both murine and human origin, with up-regulated expression levels in IL-5 transgenic mice and after inhalation of mold allergen (Huang et al., 1998). Consistent with findings in neutrophils, eosinophils derived from mice (Huang et al., 1998) and guinea pigs (Ng et al., 1991; Sun et al., 1991) also display a dose-dependent bell-shaped chemotactic response to $\mathrm{LTB}_{4}$ with an optimum concentration of 1 to $10 \mathrm{nM}$. However, species differences may exist in terms of $\mathrm{LTB}_{4}$-induced responses in eosinophils, because human cells may be less responsive to $\mathrm{LTB}_{4}$ when chemotaxis is monitored (Sun et al., 1991). Nevertheless, human blood-derived eosinophils and eosinophil-differentiated HL-60 cells exhibit an increase in intracellular calcium concentration upon stimulation with $\mathrm{LTB}_{4}$ (Patry et al., 1996; Murray et al., 2003). Furthermore, in line with findings in IL-5 transgenic mice (Huang et al., 1998), human eosinophils primed with IL-5 exhibit enhanced $\mathrm{LTB}_{4}$-induced chemotaxis (Sehmi et al., 1992). Priming with cytokines, such as IL-5, may therefore be a prerequisite for regulating the expression and responsiveness of BLT receptors on human eosinophils. In guinea pigs, $\mathrm{LTB}_{4}$ induced eosinophil superoxide generation in vitro $(\mathrm{Ng}$ et al., 1991). However, in contrast to findings in neutrophils, human eosinophil apoptosis was not altered by $\mathrm{LTB}_{4}$ (Murray et al., 2003).

There are also animal studies that support a role of $\mathrm{LTB}_{4}$ in regulating the recruitment of eosinophils into tissues in vivo. In guinea pigs, cutaneous $\mathrm{LTB}_{4}$ injection induces an accumulation of ${ }^{111}$ In-marked eosinophils (Faccioli et al., 1991) and oral treatment of sensitized guinea pigs with the selective $B^{-} \mathrm{T}_{1} \mathrm{R}$ antagonist -[6-(3hydroxy-1E,5Z-undecadienyl)-2-pyridinyl]-1,5-hexane- 
diol (U75302) decreased eosinophil influx to the lung and BAL after antigen challenge (Richards et al., 1989). However, the studies of OVA sensitization and challenge in $\mathrm{BLT}_{1} \mathrm{R}$-deficient mice have generated contradictory results, with both decreased eosinophil accumulation (Terawaki et al., 2005) and no alterations compared with results in WT mice (Miyahara et al., 2005a). In support of a pathophysiological role of $\mathrm{LTB}_{4}$-induced eosinophil activation, $\mathrm{BLT}_{1} \mathrm{R}$-deficient mice exhibit decreased leukocyte content in peritoneal exudates compared with those in WT mice in response to thioglycollate-induced acute peritonitis (Tager et al., 2000). Furthermore, oral treatment with the $\mathrm{BLT}_{1} \mathrm{R}$ antagonist CP-105,696 blocked the recruitment of eosinophils into the spinal cord and completely inhibited the development of paralysis in experimental allergic encephalomyelitis (EAE), a murine model of multiple sclerosis (MS) (Gladue et al., 1996). The potential in vivo effects of either pharmacological or genetic $\mathrm{BLT}_{1} \mathrm{R}$ targeting also reflect indirect eosinophil activation, for example, through $\mathrm{T}$ lymphocyte-dependent responses and Th2 cytokines, such as IL-5 and IL-13 (Miyahara et al., 2005a,b; Terawaki et al., 2005). Finally, findings that blood eosinophil counts are unaltered in $\mathrm{BLT}_{1} \mathrm{R}$ knockout mice compared with those in WT mice have suggested that $B T_{1} R$ is not necessary for granulocytopoiesis (Terawaki et al., 2005).

3. Basophil Granulocytes. There is presently a paucity of information regarding the expression of BLT receptors on basophil granulocytes. In human blood-derived basophils, $\mathrm{LTB}_{4}$ induces a slight chemotactic response, which is more pronounced than that observed for PAF but less than that observed with C5a (Tanimoto et al., 1992). The receptor involved in the latter response, however, is presently unknown.

4. Monocytes / Macrophages. In human blood-derived monocytes, $\mathrm{LTB}_{4}$ induces a dose-dependent increase in $\left[\mathrm{Ca}^{2+}\right]_{\mathrm{i}}$ with an $\mathrm{ED}_{50}$ of approximately $1.0 \mathrm{nM}$, which is inhibited by PTX (Rediske et al., 1993). Furthermore, $\mathrm{BLT}_{1} \mathrm{R}$ protein has been demonstrated on human bloodderived monocytes by fluorescence-activated cell sorting (Friedrich et al., 2003; Pettersson et al., 2003). An analysis of monocyte subpopulations suggested that $B_{1} T_{1} R$ protein is more abundantly expressed in monocytes with high CD14 expression compared with $\mathrm{CD} 16^{+}$monocytes (Pettersson et al., 2005).

In addition to chemotaxis, $\mathrm{BLT}_{1} \mathrm{R}$ signaling in human monocytes also induced an up-regulation of integrin expression, involved in monocyte adhesion to endothelial cells in vitro and in vivo (Vaddi and Newton, 1994; Friedrich et al., 2003). Furthermore, $\mathrm{LTB}_{4}$-induced activation of human monocytes has been associated with the release of IL-1 $\beta$ (Rola-Pleszczynski and Lemaire, 1985), IL-6 (Brach et al., 1992; Rola-Pleszczynski and Stanková, 1992), and monocyte chemoattractant protein 1 (MCP-1) (Huang et al., 2004).

$\mathrm{BLT}_{1} \mathrm{R}$ expression has also been demonstrated on peritoneal and alveolar macrophages and in RAW 264.7 cells (Huang et al., 1998; Toda et al., 1999). In the latter macrophage cell line, $\mathrm{LTB}_{4}$ induces increased proliferation and $\left[{ }^{3} \mathrm{H}\right]$ thymidine uptake, suggesting that $\mathrm{LTB}_{4}$ may also have mitogenic effects on macrophages (Nieves and Moreno, 2006).

Early studies had shown that $\mathrm{LTB}_{4}$ enhanced macrophage bacterial phagocytosis in vitro (Demitsu et al., 1989). Subsequent results have demonstrated that this effect required the cells to be opsonized with IgG and that $\mathrm{LTB}_{4}$ enhanced only $\mathrm{Fc}$ receptor $(\mathrm{FcR})$-mediated phagocytosis (Mancuso and Peters-Golden, 2000), whereas the phagocytosis of apoptotic cells (efferocytosis) was not affected by $\mathrm{LTB}_{4}$ (Canetti et al., 2003). The signaling pathways involved in $\mathrm{LTB}_{4}$-enhanced $\mathrm{FcR}$-dependent phagocytosis include $\mathrm{G} \alpha_{\mathrm{i} 3}$ protein (Peres et al., 2007), PKC activation (Mancuso and Peters-Golden, 2000), and the tyrosine kinase Syk (Canetti et al., 2003). It is noteworthy that a recent report demonstrated that FcR engagement results in tyrosine phosphorylation of $\mathrm{BLT}_{1} \mathrm{R}$ by the Src family of kinases, which leads to a molecular complex within plasma membrane lipid rafts comprising the $\mathrm{FcR}, \mathrm{BLT}_{1} \mathrm{R}, \mathrm{G} \alpha_{\mathrm{i} 3}$ protein, and Src (Serezani et al., 2009). Because disruption of lipid rafts abolished the $\mathrm{LTB}_{4}$-enhanced phagocytosis in the latter study, the formation of a complex between $B^{2} T_{1} R$ and immunoreceptors may represent a major mechanism in macrophage phagocytosis.

Several of the $\mathrm{LTB}_{4}$-induced responses in monocytes/ macrophages, such as MCP-1 secretion, intregrin-dependent monocyte arrest, and macrophage phagocytosis enhancement, are blocked by selective $\mathrm{BLT}_{1} \mathrm{R}$ antagonists (Friedrich et al., 2003; Huang et al., 2004). However, $\mathrm{BLT}_{2}$ receptor mRNA has also been detected in human monocytes (Yokomizo et al., 2001a). Furthermore, $\mathrm{BLT}_{1}$ and $\mathrm{BLT}_{2}$ receptor expression has been demonstrated immunohistochemically in macrophagerich areas within human atherosclerotic lesions (Bäck et al., 2005) and abdominal aortic aneurysms (Houard et al., 2009), as well as by in situ hybridization of macrophages in synovial tissues from patients with rheumatoid arthritis (Hashimoto et al., 2003). Although an initial study demonstrated that macrophages derived from $\mathrm{BLT}_{1} \mathrm{R}$ knockout mice did not alter $[\mathrm{Ca}]_{\mathrm{i}}$ after $\mathrm{LTB}_{4}$ stimulation (Tager et al., 2003), a subsequent study reported that these cells display chemotaxis toward $\mathrm{LTB}_{4}$ albeit at 100 -fold higher concentrations than that in WT macrophages (Subbarao et al., 2004). The latter observation suggests that the low-affinity $\mathrm{BLT}_{2}$ receptor may be involved in $\mathrm{LTB}_{4}$-induced monocyte/macrophage chemotaxis. Based on results obtained in transfected CHO cells, in which expression of one BLT receptor leads to chemotaxis toward a narrow range of $\mathrm{LTB}_{4}$ concentrations, coexpression of human $\mathrm{BLT}_{1}$ and $\mathrm{BLT}_{2}$ receptors makes cells migrate toward a wider range of $\mathrm{LTB}_{4}$ concentrations (Yokomizo et al., 2001a).

5. Dendritic Cells. Dendritic cells (DCs) are antigenpresenting cells that control adaptive immunity through 
direct interaction with $\mathrm{T}$ lymphocytes. Initially, human monocyte-derived DCs were reported to express mRNA for $\mathrm{BLT}_{2} \mathrm{R}$ but not $\mathrm{BLT}_{1} \mathrm{R}$ and the $\mathrm{LTB}_{4}$-induced $\mathrm{DC}$ chemotaxis was reported to be inhibited by the $\mathrm{BLT}_{2} \mathrm{R}$ antagonist LY255283 (Shin et al., 2006). However, a subsequent study revealed $\mathrm{BLT}_{1} \mathrm{R}$ protein expression by flow cytometric analysis of human DCs (Toda et al., 2010). The latter finding was in line with the demonstration of $\mathrm{BLT}_{1} \mathrm{R}$ but not $\mathrm{BLT}_{2} \mathrm{R}$ mRNA in murine bone marrow-derived DCs (BMDCs) (Toda et al., 2010). The latter cells exhibit functional responses to $\mathrm{LTB}_{4}$, such as increased $\left[\mathrm{Ca}^{2+}\right]_{\mathrm{i}}$ and chemotaxis (Del Prete et al., 2007; Toda et al., 2010), not observed in BMDCs derived from either $B^{-} T_{1} R$ or $B_{1} T_{1} R$ and $B_{2} T_{2} R$ knockout mice (Del Prete et al., 2007; Toda et al., 2010). Other responses associated with $\mathrm{LTB}_{4}$ stimulation of murine DCs include induction of the chemokine receptor CCR7 (Del Prete et al., 2007), IL-12 secretion, and NF- $\kappa \mathrm{B}$ translocation (Toda et al., 2010). Furthermore, murine DCs lacking both $\mathrm{BLT}_{1} \mathrm{R}$ and $\mathrm{BLT}_{2} \mathrm{R}$ exhibited decreased in vivo migration to draining lymph nodes after either paw injection (Del Prete et al., 2007) or intratracheal instillation (Miyahara et al., 2008). In vivo models have also implicated a pathophysiological role for DC BLT receptors by demonstrating decreased airway hyperreactivity after transfer of OVA-pulsed $\mathrm{BLT}_{1}$ R-deficient BMDCs compared with wild-type BMDCs (Miyahara et al., 2008) and decreased skin contact hypersensitivity in $\mathrm{BLT}_{1} \mathrm{R}$ and $\mathrm{BLT}_{2} \mathrm{R}$ knockout mice (Del Prete et al., 2007). Finally, the OVA-dependent cytokine production in mouse splenocytes after adoptive transfer of $\mathrm{BLT}_{1} \mathrm{R}$-deficient BMDCs exhibited decreased production of the Th1 cytokine IFN- $\gamma$ and elevated levels of the Th2 cytokines IL-4 and IL-5 (Toda et al., 2010).

6. T Lymphocytes. The chemotactic response of human blood-derived human $\mathrm{T}$ lymphocytes was initially reported to exhibit a peak concentration of $\mathrm{LTB}_{4}$ at $10^{-8}$ $\mathrm{M}$ (i.e., a lower potency compared with granulocytes) (Leppert et al., 1995). The latter observation was supported by the binding of radioactive $\mathrm{LTB}_{4}$ to the $\mathrm{CD} 4^{+} 8^{+} 3^{\text {low }}$ Tsup- 1 cell line, which was characterized by a $K_{\mathrm{d}}$ of approximately $200 \mathrm{nM}$ and a rank order of competitive potencies of $20-\mathrm{OH}-\mathrm{LTB}_{4}, 6$-trans-LTB ${ }_{4}$, which were both similar to that of the low-affinity subset of human neutrophil $\mathrm{LTB}_{4}$-binding sites (Leppert et al., 1995). Subsequent studies of peripheral blood cells have supported those initial observations by the identification of dominant levels of $\mathrm{BLT}_{2} \mathrm{R}$ mRNA compared with $\mathrm{BLT}_{1} \mathrm{R}$ mRNA (Yokomizo et al., 2001a). Moreover, in peripheral blood, $\mathrm{LTB}_{4}$ bound only to $11 \%$ of T lymphocytes, representing mainly cells of the $\mathrm{CD}^{+}$subset (Payan et al., 1984). The latter observation was subsequently supported by a flow cytometric demonstration of $\mathrm{BLT}_{1}$ and CD8 coexpression in a small fraction of peripheral blood cells, whereas $\mathrm{CD}^{+}$lymphocytes were $\mathrm{BLT}_{1} \mathrm{R}$-negative (Pettersson et al., 2003). However, decreased $\mathrm{BLT}_{2} \mathrm{R}$ expression has been observed after $\mathrm{T}$ - lymphocyte stimulation (Yokomizo et al., 2001a), and animal studies indicate that $\mathrm{BLT}_{1} \mathrm{R}$ activation may be the major signaling pathway mediating $\mathrm{LTB}_{4}$-induced $\mathrm{T}$ lymphocyte recruitment in vivo (Goodarzi et al., 2003; Tager et al., 2003; Miyahara et al., 2005a). Furthermore, morphological analyses have associated adventitial vascular inflammation with an accumulation of $\mathrm{BLT}_{1} \mathrm{R}$ expressing T lymphocytes (Houard et al., 2009).

$\mathrm{BLT}_{1} \mathrm{R}$ mRNA expression is induced in antigen-exposed murine $\mathrm{CD} 8^{+}$T-effector cells, associated with an increased chemotactic response; effects inhibited either by the $\mathrm{BLT}_{1} \mathrm{R}$ antagonist CP-105,696 or in cells derived from $\mathrm{BLT}_{1} \mathrm{R}$ knockout mice (Goodarzi et al., 2003; Ott et al., 2003). These findings suggest that the ability to use $\mathrm{BLT}_{1} \mathrm{R}$ for migration into inflamed tissues is a feature specific to fully differentiated $\mathrm{CD}^{+}$effector cells (Goodarzi et al., 2003). Likewise, $\mathrm{BLT}_{1} \mathrm{R}$ mRNA is induced in $\mathrm{CD}^{+}$murine lymphocytes during either Th1 or Th2 polarization (Tager et al., 2003). A subsequent study of human blood-derived $\mathrm{T}$ lymphocytes demonstrated that the small fraction of $\mathrm{BLT}_{1} \mathrm{R}$-positive cells also were enriched for the activation markers CD38 and HLA-DR and expressed the effector cytokines IFN- $\gamma$ and IL-4 (Islam et al., 2006). Furthermore, in vitro activation of human T lymphocytes by alloactivated DCs increased $\mathrm{BLT}_{1} \mathrm{R}$ surface expression and enhanced $\mathrm{LTB}_{4}$-induced chemotaxis (Islam et al., 2006). In further support of an up-regulation of $\mathrm{BLT}_{1} \mathrm{R}$ in activated $\mathrm{T}$ lymphocytes, the number of circulating $\mathrm{CD}^{+}$cells positive for $\mathrm{BLT}_{1} \mathrm{R}$ was increased during acute Epstein-Barr virus infection (Islam et al., 2006). Finally, bronchoalveolar lavage derived from patients with lung transplant graft dysfunction (Medoff et al., 2005) and asthma (Islam et al., 2006) contained an increased proportion of $\mathrm{BLT}_{1} \mathrm{R}$-positive $\mathrm{T}$ lymphocytes.

$\mathrm{LTB}_{4}$ stimulation also enhanced the production of IL-1, IL-2, IL-5, and IFN- $\gamma$ in human blood-derived T lymphocytes in vitro (Rola-Pleszczynski et al., 1986; Yamaoka and Kolb, 1993) and the production of MMP-2, -3 , and -9 in $\mathrm{CD}^{+} 8^{+} 3^{\text {low }}$ Tsup- 1 cells (Leppert et al., 1995). Furthermore, $\mathrm{LTB}_{4}$ inhibited the differentiation of murine naive $\mathrm{CD} 4^{+} \mathrm{T}$ lymphocytes into $\mathrm{CD} 25^{+}$ Foxp $3^{+} \mathrm{T}$ regulatory cells and increased IL-17 expression (Chen et al., 2009a), providing the first suggestion that $\mathrm{LTB}_{4}$ may counteract the generation of immunosuppressive $\mathrm{T}$ regulatory cells and promote the differentiation into Th17 cells. In support of a role for $\mathrm{LTB}_{4}$ in the development of Th17 immune responses, lymph node cells derived from $\mathrm{BLT}_{1} \mathrm{R}$ knockout mice after EAE induction exhibit decreased IL-17 production (Kihara et al., 2010).

The role of BLTR signaling in T lymphocyte recruitment has also received support from in vivo studies. The pulmonary inflammation and airway hyperresponsiveness after OVA sensitization and challenge are reduced in $\mathrm{BLT}_{1} \mathrm{R}$ knockout mice and restored after reconstitution with $\mathrm{BLT}_{1} \mathrm{R}$-expressing $\mathrm{T}$ lymphocytes (Tager et al., 
2003; Miyahara et al., 2005a). In addition, mice lacking $\mathrm{CD}^{+} \mathrm{T}$ lymphocytes are protected from airway hyperresponsiveness induced by allergen sensitization and challenge (Miyahara et al., 2005b). Whereas reinstallation of $\mathrm{CD}^{+}$cells restored airway hyperresponsiveness in the latter mice, adoptive transfer of $\mathrm{BLT}_{1} \mathrm{R}$-deficient $\mathrm{CD}^{+}$cells maintained the protective effect (Miyahara et al., 2005b). In other models, such as complete Freund's adjuvant-induced peritonitis, adoptive transfer of fluorescence-marked T lymphocytes derived from both $\mathrm{BLT}_{1} \mathrm{R}$-deficient and WT mice resulted in an accumulation of 1.6- to 1.9-fold higher numbers of WT cells in peritoneal exudates (Goodarzi et al., 2003).

7. B Lymphocytes. $\mathrm{BLT}_{1} \mathrm{R}$ protein and $\mathrm{BLT}_{1} \mathrm{R}$ and $\mathrm{BLT}_{2} \mathrm{R}$ mRNA have been detected in $\mathrm{CD}_{1}{ }^{+}$human $\mathrm{B}$ lymphocytes (Yokomizo et al., 2001a; Pettersson et al., 2003; Runarsson et al., 2005). In cells derived from either blood or tonsillectomy tissues, $\mathrm{LTB}_{4}$ enhances the effects of other stimulating factors on CD23 expression, proliferation, and differentiation of resting, but not activated, B lymphocytes (Yamaoka et al., 1989, 1994; Dugas et al., 1990). The latter findings were subsequently supported by the demonstration that EpsteinBarr virus infection of $B$ lymphocytes is associated with a loss of $\mathrm{BLT}_{1} \mathrm{R}$ expression and a lack of $\mathrm{LTB}_{4}$-induced effects on the proliferation of infected cells (Liu et al., 2008). $\mathrm{BLT}_{1} \mathrm{R}$ protein expression has also been demonstrated in B lymphocytes derived from patients with B-cell chronic lymphocytic leukemia (Runarsson et al., 2005). In this study, LT synthesis inhibitors decreased DNA synthesis and antigen expression in B-cell chronic lymphocytic leukemia lymphocytes, an effect that was reversed by exogenously added $\mathrm{LTB}_{4}$ (Runarsson et al., 2005).

8. Mast Cells. Experiments in human umbilical cord blood-derived mast cells have demonstrated that $\mathrm{LTB}_{4}$ is a chemoattractant for the immature c-kit ${ }^{+} \mathrm{MC}$ populations, whereas the mature c-kit ${ }^{\text {high }} \mathrm{MCs}$ are unresponsive (Weller et al., 2005). The chemotactic effects induced by $\mathrm{LTB}_{4}$ on murine bone marrow-derived mast cells (BMMCs) cultured in the presence of IL-3 may also depend on the maturity of the MC, because cells cultured for 2 to 6 weeks migrate in response to $\mathrm{LTB}_{4}$ (Kitaura et al., 2005; Weller et al., 2005; Lundeen et al., 2006), whereas after 10 weeks of culture, the cells are unresponsive to this agonist (Weller et al., 2005). The latter finding correlated with a decrease in $\mathrm{BLT}_{1} \mathrm{R}$ mRNA. For example, 2-week-old BMMCs expressed 4-fold higher levels than 6-week-old cells and 10-fold more than 10-week-old cells (Weller et al., 2005). A subsequent study also described $\mathrm{BLT}_{2} \mathrm{R}$ mRNA in BMMC after 4 weeks of culture and indicated that either the $\mathrm{BLT}_{1} \mathrm{R}$ antagonist $\mathrm{U} 75302$ or the $\mathrm{BLT}_{2}$ receptor antagonist LY255283 inhibited $\mathrm{LTB}_{4}$-induced BMMC chemotaxis, although no additive effect was observed after combination of the two receptor antagonists (Lundeen et al., 2006). In addition, antigen stimulation in- creased $\mathrm{BLT}_{2} \mathrm{R}$ expression in murine BMMCs (Cho et al., 2010a). In support of a functional murine $\mathrm{MC} \mathrm{BLT}_{2}$ receptor, the $\mathrm{BLT}_{2}$ receptor agonists $12-\mathrm{HHT}$ and $12(\mathrm{~S})$ HETE have also been reported to induce BMMC migration (Lundeen et al., 2006; Okuno et al., 2008). The $\left[\mathrm{Ca}^{2+}\right]_{\mathrm{i}}$ induced by 12 -HHT was abolished in BMMCs derived from $\mathrm{BLT}_{2} \mathrm{R}$ knockout mice, whereas BMMCs from both WT and $\mathrm{BLT}_{1} \mathrm{R}$ knockout mice responded to $\mathrm{LTB}_{4}$ (Mathis et al., 2010). However, $\mathrm{LTB}_{4}$-induced migration was abolished in BMMCs derived from $\mathrm{BLT}_{1} \mathrm{R}$ deficient mice, whereas 12-HHT-induced migration was abolished in BMMCs derived from $\mathrm{BLT}_{2} \mathrm{R}$-deficient mice (Okuno et al., 2008). Finally, a human MC line, HMC-1, has been reported to express both $\mathrm{BLT}_{1}$ and $\mathrm{BLT}_{2}$ receptor mRNA and migrate in response to $\mathrm{LTB}_{4}$ (Lundeen et al., 2006). An unexpected finding was that flow cytometry and immunostaining suggested an intracellular localization of $\mathrm{BLT}_{1} \mathrm{R}$ protein in these cells (Lundeen et al., 2006), but the functional significance of this receptor localization remains to be established.

9. Vascular Smooth Muscle Cells. The $\mathrm{LTB}_{4}$-induced vasoconstriction, demonstrated in human pulmonary artery (Sakata et al., 2004), in guinea pig pulmonary artery and aorta (Bäck et al., 2004; Sakata et al., 2004), and in rat basilar artery (Trandafir et al., 2005), has led to the hypothesis that nonmyeloid cells, such as SMCs, also express BLT receptors. Immunohistochemical analysis of human arteries has revealed $\mathrm{BLT}_{1} \mathrm{R}$ expression in the muscular layers of carotid atherosclerotic endarterectomies, as well as in human nonatheroclerotic mammary arteries (Bäck et al., 2005). Moreover, in human coronary artery SMCs, 8-fold higher BLT $_{1} \mathrm{R}$ mRNA levels compared with $\mathrm{BLT}_{2} \mathrm{R}$ mRNA has been demonstrated by RT-PCR (Heller et al., 2005), supporting the observation that the $\mathrm{BLT}_{1} \mathrm{R}$ subtype may be the dominant BLT receptor subtype in vascular SMCs. The expression of $\mathrm{BLT}_{1} \mathrm{R}$ proteins on human coronary artery SMCs has also been demonstrated by Western blot (Bäck et al., 2005) and flow cytometry (Heller et al., 2005). In contrast to this apparent constitutive $B T_{1} R$ expression in human coronary artery SMCs, murine aortic SMCs express BLT $_{1} \mathrm{R}$ mRNA only after $24 \mathrm{~h}$ of treatment with TNF- $\alpha$ and IFN- $\gamma$ (Heller et al., 2005).

In human coronary artery $\mathrm{SMCs}, \mathrm{LTB}_{4}$ induced an approximately 4-fold increase in whole-cell currents measured by patch clamp, and the activation of SMC by $\mathrm{LTB}_{4}$ induces migration and proliferation (Bäck et al., 2005). Furthermore, $\mathrm{LTB}_{4}$ stimulated the release of MMP-2 (Hlawaty et al., 2009; Seo et al., 2010) and activation of integrin signaling pathways in vascular SMCs (Moraes et al., 2010). The pathophysiological importance of $\mathrm{LTB}_{4}$-induced activation of SMCs in the context of atherosclerosis has been reinforced by the inhibitory effects of BLT receptor antagonists on the development of intimal hyperplasia after vascular injury (Kondo et al., 1998; Bäck et al., 2005; Hlawaty et al., 2009). Furthermore, atherosclerotic lesions of apoE $(-/-)$ mice 
display reduced SMC content after $\mathrm{BLT}_{1} \mathrm{R}$ gene disruption (Heller et al., 2005).

10. Bronchial Smooth Muscle Cells. In line with findings in human vessels, immunohistochemical staining of human bronchi revealed colocalization of both $\mathrm{BLT}_{1} \mathrm{R}$ and $\mathrm{BLT}_{2} \mathrm{R}$ protein with smooth muscle $\alpha$-actin-positive airway SMCs (Watanabe et al., 2009). Furthermore, both receptors were also identified on human airway SMCs in culture by RT-PCR, Western blot analysis, and flow cytometry. In the latter cells, $\mathrm{LTB}_{4}$ induced proliferation and migration, which was inhibited by the selective $\mathrm{BLT}_{1} \mathrm{R}$ antagonist U75302 (Watanabe et al., 2009).

11. Endothelial Cells. In human vessels, endothelial expression of $\mathrm{BLT}_{1} \mathrm{R}$ has been demonstrated by immunohistochemical analysis and was only observed in atherosclerotic and not in healthy arteries, suggesting an induction during vascular inflammation (Bäck et al., 2005). The latter notion of $\mathrm{BLT}_{1} \mathrm{R}$ immunoregulation has received support from studies in HUVECs demonstrating $\mathrm{BLT}_{1} \mathrm{R}$ up-regulation by LPS and IL- $1 \beta$ (Table 2 ), whereas $\mathrm{BLT}_{2} \mathrm{R}$ was induced by TNF- $\alpha$ (Qiu et al., 2006).

$\mathrm{LTB}_{4}$-induced endothelial activation has also been implicated in increased leukocyte adherence (Hoover et al., 1984). However, the in vitro neutrophil adherence observed after $\mathrm{LTB}_{4}$ stimulation of HUVECs is weak (Lindström et al., 1990; Palmblad et al., 1994), and the role of endothelial BLT receptors in leukocyte adhesion is today not fully characterized. Papayianni et al. (1996) demonstrated that $\mathrm{LTB}_{4}$ had no effect on P-selectin expression in HUVECs. Another study reported no effect on monocyte arrest under physiological flow conditions after EC stimulation with $\mathrm{LTB}_{4}$ (Friedrich et al., 2003). These observations provided evidence for a direct effect of $\mathrm{LTB}_{4}$ on leukocytes rather than on ECs. However, other investigators demonstrated that the endothelium released vasoactive factors via BLT receptor activation (Bäck et al., 2004; Qiu et al., 2006). These reports provide evidence for a role of $\mathrm{LTB}_{4}$ in regulating endothelial function. In addition, vascular endothelial growth factor (VEGF) up-regulated the expression of $\mathrm{BLT}_{1} \mathrm{R}$ and $\mathrm{BLT}_{2} \mathrm{R}$ mRNA and protein in HUVECs (Kim et al., 2009). It is noteworthy that VEGF also increased the release of $12(S)$-HETE, and the VEGF-induced blood vessel formation in vivo and in vitro was inhibited by either the $\mathrm{BLT}_{2} \mathrm{R}$ antagonist LY255283 or $\mathrm{BLT}_{2} \mathrm{R}$ knock down by small interfering RNA (Kim et al., 2009). These findings suggest a role for 12(S)-HETE signaling through endothelial $\mathrm{BLT}_{2} \mathrm{R}$ in angiogenesis. Taken together, the data indicate that the ligands for the BLT receptors affect ECs but that the interaction between the ligands and the endothelium in adhesion experiments is presently difficult to observe, possibly being masked by the $\mathrm{LTB}_{4}$-induced effects on leukocytes.

\section{E. BLT Receptor Functional Analysis through Altered Gene Expression}

1. BLT Receptor Transgenic Models. Transgenic mice expressing the human $\mathrm{BLT}_{1} \mathrm{R}$ under the $\mathrm{CD} 11 \mathrm{~b}$ promoter to obtain leukocyte specific expression exhibit an increased granulocyte infiltration in response to topical $\mathrm{LTB}_{4}$ application and ischemia reperfusion (Chiang et al., 1999; Brink et al., 2003). In addition, transgenic $\mathrm{BLT}_{2} \mathrm{R}$ overexpressed in mice have been studied in a model of angiogenesis and shown to exhibit increased blood vessel formation in response to $\mathrm{LTB}_{4}$ and $12(S)$-HETE (Kim et al., 2009).

2. BLT $T_{1}$ Receptor Knockout. Mice lacking $\mathrm{BLT}_{1} \mathrm{R}$ were generated in 2000 (Haribabu et al., 2000; Tager et al., 2000; Brink et al., 2003). Although these mice developed normally and had no apparent hematopoietic abnormalities, decreased leukocyte chemotaxis (Haribabu et al., 2000; Tager et al., 2003) and integrin-mediated leukocyte arrest in postcapillary venules (Tager et al., 2003) in response to $\mathrm{LTB}_{4}$ compared with WT mice were reported. In addition, $\mathrm{BLT}_{1} \mathrm{R}$ deficiency conferred protection against inflammatory responses in different disease models.

After induction of acute peritonitis, $\mathrm{BLT}_{1} \mathrm{R}$-deficient mice exhibit decreased leukocyte content in peritoneal exudates compared with that in WT mice (Haribabu et al., 2000; Tager et al., 2000; Goodarzi et al., 2003) Other in vivo studies of endotoxin provocation have demonstrated decreased portal venule leukocyte adherence in response to systemic LPS in $B_{1} T_{1} R$ knockout mice compared with that in WT mice (Ito et al., 2008), whereas the leukocyte infiltration after local intraocular LPS injection was not altered by $\mathrm{BLT}_{1} \mathrm{R}$ disruption (Smith et

TABLE 2

Immunoregulation of human $B L T_{1} R$ expression

\begin{tabular}{|c|c|c|c|c|}
\hline Receptor & Cytokine & Cell & Effect $^{a}$ & Reference \\
\hline \multirow{11}{*}{$\mathrm{BLT}_{1}$} & IFN- $\gamma$ & Monocytes & $\downarrow$ mRNA; $\downarrow$ protein; $\downarrow$ chemotaxis & Pettersson et al., 2005 \\
\hline & $\mathrm{TNF}-\alpha$ & Monocytes & $\downarrow$ mRNA; $\downarrow$ protein & Pettersson et al., 2005 \\
\hline & & Granulocytes & $\downarrow$ protein & Pettersson et al., 2003 \\
\hline & IL-1 $\beta$ & Vascular SMCs & $\uparrow$ mRNA & Bäck et al., 2005 \\
\hline & & HUVECs & $\uparrow$ mRNA; $\uparrow$ protein & Qiu et al., 2006 \\
\hline & IL-10 & Monocytes & $\uparrow$ mRNA; $\uparrow$ protein & Pettersson et al., 2005 \\
\hline & LPS & Monocytes & $\downarrow$ mRNA & Pettersson et al., 2005 \\
\hline & & Granulocytes & $\downarrow$ protein & Pettersson et al., 2003 \\
\hline & & Vascular SMCs & $\uparrow \mathrm{mRNA}$ & Bäck et al., 2005 \\
\hline & & HUVECs & $\uparrow \operatorname{mRNA} ; \uparrow$ protein; $\uparrow\left[\mathrm{Ca}^{2+}\right]_{\mathrm{i}}$ & Qiu et al., 2006 \\
\hline & IL-5 & Eosinophils & $\uparrow$ chemotaxis & Sehmi et al., 1992; Thivierge et al., 2000 \\
\hline
\end{tabular}

${ }^{a} \uparrow$, up-regulation; $\downarrow$, down-regulation; specified are the effects (at mRNA, protein, and functional level). 
al., 2004). In addition to models of acute inflammation, $\mathrm{BLT}_{1} \mathrm{R}$ knockout mice have also revealed decreased inflammation in more chronic inflammatory responses. For example, in contrast to the lack of effects on endotoxin-induced uveitis, $\mathrm{BLT}_{1} \mathrm{R}$-deficient mice were protected from experimental autoimmune uveitis induced by immunization of interphotoreceptor retinoid-binding protein (Liao et al., 2006).

Mice lacking apolipoprotein E exhibit hyperlipidemia and develop spontaneous atherosclerosis. Apolipoprotein $\mathrm{E}$ and $\mathrm{BLT}_{1} \mathrm{R}$ double knockout mice display smaller lesions compared with their apoE $(-/-)$ littermates (Subbarao et al., 2004; Heller et al., 2005; Bäck, 2008b). The latter double knockout mice are also protected from abdominal aortic aneurysm development induced by angiotensin II infusion (Ahluwalia et al., 2007).

After OVA sensitization, $\mathrm{BLT}_{1} \mathrm{R}$ knockout mice exhibit decreased airway responsiveness associated with a decreased leukocyte accumulation (Tager et al., 2003; Miyahara et al., 2005a; Terawaki et al., 2005), which is restored after adoptive transfer of $\mathrm{BLT}_{1} \mathrm{R}$-expressing $\mathrm{T}$ lymphocytes (Miyahara et al., 2005a; Tager et al., 2003) (see section II.D). In the EAE model, $\mathrm{BLT}_{1}$ R-deficient mice develop less severe clinical signs of disease and a significantly delayed onset of disease than WT mice, associated with decreased inflammatory infiltration in the spinal cord (Kihara et al., 2010). Finally, BLT ${ }_{1} R$ knockout mice are protected in models of both collagenand $\mathrm{K} / \mathrm{BxN}$ serum-induced arthritis (Kim et al., 2006a; Shao et al., 2006; Mathis et al., 2010), discussed in detail below. In this context, $\mathrm{BLT}_{1} \mathrm{R}$ knockout mice displayed a decrease in bone resorption induced by either LPS or ovariectomy (Hikiji et al., 2009).

3. BLT $T_{2}$ Receptor Knockout. In recent studies, $\mathrm{BLT}_{2} \mathrm{R}$ knockout mice were generated (Iizuka et al., 2010; Mathis et al., 2010). Because of to the close proximity of the $B_{1} T_{1} R$ gene promoter, the $B_{2} R$ gene disruption was generated through insertion into the open reading frame of the $\mathrm{BLT}_{2}$ gene to avoid alterations of $\mathrm{BLT}_{1} \mathrm{R}$ expression (Iizuka et al., 2010; Mathis et al., 2010). The $\mathrm{BLT}_{2} \mathrm{R}$-deficient mice were viable, developed normally, and displayed no overt behavioral or morphological defects (Mathis et al., 2010). An unexpected finding was that opposing phenotypic changes were observed in two different mouse models of disease. $\mathrm{BLT}_{2} \mathrm{R}$ knockout mice displayed attenuated inflammatory arthritis in response to $\mathrm{K} / \mathrm{BxN}$ serum transfer (Mathis et al., 2010). The latter effect was associated with decreased inflammatory cell influx and could be reproduced by bone marrow transplantation, suggesting that leukocyte $\mathrm{BLT}_{2} \mathrm{R}$ is necessary for full arthritis development (Mathis et al., 2010). However, the protection against $\mathrm{K} / \mathrm{BxN}$ serum-induced arthritis conferred by the lack of $\mathrm{BLT}_{1} \mathrm{R}$ is more pronounced compared with that observed in $\mathrm{BLT}_{2} \mathrm{R}$-deficient mice (Kim et al., 2006a; Mathis et al., 2010). Nevertheless, the transfer of WT neutrophils into $\mathrm{BLT}_{1} \mathrm{R}$ knockout mice promoted the entry of endogenous $\mathrm{BLT}_{1} \mathrm{R}$-deficient neutrophils into the joints of these mice (Kim et al., 2006a) through the production of IL-1 (Chou et al., 2010). Taken together, these data suggested that although the $B_{1} T_{1} R$ may be necessary for the initiation of autoantibody-induced arthritis, $\mathrm{BLT}_{2} \mathrm{R}$ may play a possible role at later stages of disease, when local $\mathrm{LTB}_{4}$ concentrations are higher in the joint (Mathis et al., 2010). In contrast to the findings of decreased arthritis in $\mathrm{BLT}_{2} \mathrm{R}$ knockout mice, another study demonstrated that this gene disruption induced more severe colitis in response to dextran sodium sulfate (DSS) compared with that in either WT or BLT $\mathrm{R}_{1}$ knockout mice, which was accompanied by increased expression of inflammatory cytokines, chemokines, and MMPs (Iizuka et al., 2010). However, in the latter study, $\mathrm{BLT}_{2} \mathrm{R}$ deficiency was associated with a dysfunctional barrier function in colonic epithelial cells rather than direct effects on leukocytes (Iizuka et al., 2010). Although the effects of DSS-induced colitis have not been fully explored in $\mathrm{BLT}_{1} \mathrm{R}$ knockout mice, those mice exhibit weight loss similar to that in WT mice, suggesting no protective effects. The notion that $\mathrm{BLT}_{2} \mathrm{R}$ agonists other than $\mathrm{LTB}_{4}$ may be involved in the exacerbated colitis observed after $\mathrm{BLT}_{2} \mathrm{R}$ knockout (Iizuka et al., 2010) is supported by the fact that HHT is produced at 5to 6-fold higher levels compared with $\mathrm{LTB}_{4}$ in inflamed colonic biopsy specimens (Zijlstra et al., 1992, 1993) (see section II.E).

4. $B L T_{1}$ and $B L T_{2}$ Receptor Double Knockout. Double knockout of $\mathrm{BLT}_{1} \mathrm{R}$ and $\mathrm{BLT}_{2} \mathrm{R}$ has been generated through direct targeting of the genes encoding both receptors (Shao et al., 2006). In line with findings after disruption of any of the BLT receptor subtypes alone (see above), these mice were also viable, developed normally, and displayed no overt behavioral or morphological defects and no alterations of leukocyte subpopulation counts (Shao et al., 2006). After collagen-induced arthritis, both $\mathrm{BLT}_{1} \mathrm{R}$ knockout and $\mathrm{BLT}_{1} \mathrm{R}_{-\mathrm{BLT}_{2} \mathrm{R}}$ knockout mice respond in a similar fashion with less clinical and histological signs of arthritis, as well as smaller synovial inflammatory cell infiltration compared with WT mice, without altering the antibody response (Shao et al., 2006).

\section{F. Potential Therapeutic Applications}

The cell-type specific BLTR signaling discussed above (see section II.D) may imply differential effects of $\mathrm{LTB}_{4}$ in the development of different diseases. For example, $\mathrm{LTB}_{4}$ may mainly exert its effects on neutrophil granulocytes in models of arthritis (Kim et al., 2006a) abdominal aortic aneurysms (Houard et al., 2009), and cerebral ischemia/reperfusion (Barone et al., 1992), whereas models of MS have implicated a major role of $\mathrm{LTB}_{4}{ }^{-}$ induced effects on eosinophil granulocytes (Gladue et al., 1996). In contrast, T lymphocytes may be the main effector cells in $\mathrm{LTB}_{4}$-induced airway hyperresponsiveness (Miyahara et al., 2005a). Furthermore, in other 
disease models, $\mathrm{LTB}_{4}$ signaling may affect several cell types, such as in atherosclerosis, in which macrophages, vascular SMCs, and ECs express $\mathrm{BLT}_{1}$ receptors (Bäck et al., 2005). The roles of LTs and related lipid mediators in various diseases have been extensively reviewed (Shimizu, 2009).

1. Atherosclerosis. The uptake and modification of lipids in the vascular wall induce a local inflammatory reaction, eventually developing into an atherosclerotic lesion. Generation of $\mathrm{LTB}_{4}$ has been described in ex vivo stimulated atherosclerotic vessels, and intraluminal LT formation has been demonstrated during coronary balloon angioplasty (Brezinski et al., 1992; Bäck, 2008b). In addition, several of the BLT receptor-expressing immune cells discussed above are present in the atherosclerotic lesion (Bäck, 2008b). Although studies of targeted 5 -LO have generated contradictory results, $\mathrm{BLT}_{1} \mathrm{R}$ knock out (Subbarao et al., 2004; Heller et al., 2005) and the $\mathrm{BLT}_{1} \mathrm{R}$ antagonist CP-105,696 (Aiello et al., 2002) reduces atherosclerosis in hyperlipidemic mice (Bäck, $2008 \mathrm{~b})$. In addition to effects on the inflammatory response, $\mathrm{LTB}_{4}$ signaling through $\mathrm{BLT}_{1} \mathrm{R}$ expressed in vascular SMCs may also be involved in the development of atherosclerosis and intimal hyperplasia after vascular interventions (Bäck et al., 2005; Heller et al., 2005; Hlawaty et al., 2009). Clinical trials have been initiated to evaluate the effects of anti-LTs on biomarkers and atherosclerosis morphology in patients with coronary heart disease (Bäck, 2009a).

2. Aortic Abdominal Aneurysms. Circulating neutrophils derived from patients undergoing surgery for abdominal aortic aneurysms produce increased levels of $\mathrm{LTB}_{4}$ (Gadaleta et al., 1994), and the expression levels of $\mathrm{LTB}_{4}$ synthesizing enzymes has in addition been demonstrated in neutrophils infiltrating the intraluminal thrombus covering the aneurysm (Houard et al., 2009). This local $\mathrm{LTB}_{4}$ production has also been demonstrated to transduce the major part of the chemotactic activity derived from the intraluminal thrombus (Houard et al., 2009). In addition to neutrophils, BLTR-positive macrophages and $\mathrm{T}$ lymphocytes may be involved in the adventitial inflammation, which is part of the pathogenesis of abdominal aortic aneurysms (Houard et al., 2009). The importance of $\mathrm{BLT}_{1} \mathrm{R}$ signaling has also received support from animal studies. Either genetic or pharmacological targeting of $\mathrm{BLT}_{1} \mathrm{R}$ signaling reduces the incidence of experimental abdominal aortic aneurysms induced by angiotensin infusion in apoE knockout mice (Ahluwalia et al., 2007; Kristo et al., 2010).

3. Cerebrovascular Disease. Leukotrienes are produced during cerebral ischemia, and LT synthesis inhibitors limit the damage after experimental cerebral ischemia and reperfusion (Bäck, 2009b). Although most studies on the receptors involved in this protective effect have focused on CysLTRs and more recently GPR17 (see section III.H), there are also studies supporting a role for BLTR signaling in cerebrovascular disease. In a study in rats, temporary middle cerebral artery occlusion followed by reperfusion time dependently increased LTB4 binding in the cerebral cortex, which was paralleled by increases in myeloperoxidase activity (Barone et al., 1992). The results of the latter study suggested increased $\mathrm{LTB}_{4}$ binding to receptors located on accumulating neutrophils in ischemic brain tissue (Barone et al., 1992).

In human cerebrovascular disease, macrophage 5-LO expression and $\mathrm{LTB}_{4}$ production have been reported to correlate with either clinical or radiological signs of cerebral ischemia in patients undergoing vascular surgery for carotid artery stenosis (Cipollone et al., 2005). Furthermore, $B T_{1} R$ and $B_{2 T} R$ are expressed within carotid artery atherosclerotic lesions (Bäck et al., 2005). Finally, and as stated above (see section II.A), haplotypes within the LTB4R and LTB4R2 gene complex conferred a 2.3 -fold increased risk for ischemic stroke in two case-control studies (Bevan et al., 2008).

4. Multiple Sclerosis. MS is an inflammatory disorder of the central nervous system associated with bloodbrain barrier breakdown, inflammatory cell accumulation, and myelin degradation (Mirshafiey and Jadidi-Niaragh, 2010). As discussed above (see section II.D), the beneficial effects on EAE after either genetic or pharmacological $\mathrm{BLT}_{1} \mathrm{R}$ targeting (Fretland et al., 1991; Gladue et al., 1996; Kihara et al., 2010) suggest that that $B_{1} T_{1} R$ signaling may potentially affect both the onset and severity of MS. In support of this, increased levels of $\mathrm{LTB}_{4}$ have been detected in cerebrospinal fluid from patients with MS (Neu et al., 1992, 2002). Of interest, 5-LO was identified as one of the most up-regulated genes by microarray analysis in both human MS lesions and in brains from mice after EAE induction (Whitney et al., 2001). In the brain parenchyma around MS lesions, 5-LO expression colocalized with macrophages (Whitney et al., 2001). However, in contrast to findings in $B_{1} T_{1} R$ knockout mice (Kihara et al., 2010), 5-LO-deficient mice exhibit an exacerbated EAE compared with WT mice (Emerson and LeVine, 2004). These opposing effects of targeting either LT synthesis or LT receptors are in line with findings in atherosclerosis (see above) and point to the fact that targeting 5-LO will inhibit both proinflammatory mediators (i.e., LTs) and anti-inflammatory mediators (i.e., lipoxins), as has been demonstrated in 5-LO-deficient mice (Bafica et al., 2005). This, in turn, suggests that endogenous anti-inflammatory mediators derived from 5-LO metabolism may be involved in regulating these disease models. In addition, studies in mice lacking 12/15-LO have also shown exacerbated EAE (Emerson and LeVine, 2004) or a defect in both corneal reepithelization and neutrophil recruitment that correlates with a reduction in endogenous lipoxin formation (Gronert et al., 2005), raising the notion that lipoxins, for which the biosynthesis involves both 5-LO and 12/15-LO (Serhan et al., 2008), may be beneficial in MS. These findings suggest that BLT receptor antago- 
nism may represent an advantage compared with 5-LO inhibition in MS.

5. Arthritis. BLTR expression has been demonstrated in synovial tissues derived from patients with rheumatoid arthritis (Hashimoto et al., 2003). The beneficial effects in models of rheumatoid arthritis after genetic disruption of $\mathrm{BLT}_{1} \mathrm{R}$ and/or $\mathrm{BLT}_{2} \mathrm{R}$ in C57BL6 mice (Kim et al., 2006a; Shao et al., 2006; Mathis et al., 2010) were discussed above (see section II.E. Pharmacological treatment with the $\mathrm{BLT}_{1} \mathrm{R}$ antagonist CP105,696 reduced the histological signs of collagen-induced arthritis in the more arthritis-prone DBA/1J mice (Griffiths et al., 1995). In humans, oral administration of the BLT receptor antagonist amebulant (Birke et al., 2001) inhibits Mac-1 expression on neutrophils (Alten et al., 2004), but clinical trials in patients with arthritis have not shown any statistically significant beneficial effects (Díaz-González et al., 2007).

6. Pulmonary Inflammation. Although most studies of LTs in asthma have focused on CysLT receptor signaling (see section III.A), several studies point to a participation of $\mathrm{LTB}_{4}$ in chronic airway inflammation. For example, whereas CysLT $_{1} R$ antagonists generally have small effects on the increased bronchial responsiveness in asthma, clinical trials of either 5-LO inhibitors or FLAP antagonists have been more effective, supporting an involvement of $\mathrm{LTB}_{4}$ (Dahlén, 2006). In addition, neutrophil recruitment to the airway is thought to be a major component of continuing inflammation and progression of chronic obstructive pulmonary disease (COPD), and the $46 \%$ of the neutrophil chemotactic activity in sputum derived from patients with COPD is inhibited by a BLT receptor antagonist (Woolhouse et al., 2002). Increased concentrations of $\mathrm{LTB}_{4}$ have also been reported in BAL (Wenzel et al., 1995) and exhaled breath condensates from patients with asthma (Csoma et al., 2002). In further support of a role of $B^{2} T_{1} R$ signaling in asthma, the $\mathrm{BLT}_{1} \mathrm{R}$ antagonist CP-105,696 decreased airway hyperresponsiveness in a primate model of asthma (Turner et al., 1996). As discussed above (section II.E), BLT $_{1} \mathrm{R}$ knockout mice exhibited decreased airway responsiveness after OVA sensitization and challenge, associated with decreased pulmonary inflammation and mucus secretion compared with that in WT mice (Miyahara et al., 2005a; Terawaki et al., 2005). In addition, $\mathrm{BLT}_{2} \mathrm{R}$ signaling may be involved in the response to OVA sensitization and challenge, as suggested by the reduced airway inflammation and reduced airway hyperresponsiveness after $\mathrm{BLT}_{2} \mathrm{R}$ targeting through in vivo administration of either the $\mathrm{BLT}_{2} \mathrm{R}$ antagonist LY255283 or an antisense $\mathrm{BLT}_{2} \mathrm{R}$ (Cho et al., 2010b).

In a crossover study of 12 atopic asthmatic individuals, the BLT receptor antagonist LY293111, 2-[2-propyl3-[3-[2-ethyl-4-(4-fluorophenyl)-5-hydroxyphenoxy]propoxy]phenoxyl]benzoic acid (LY293111) did not alter either lung function or airway reactivity after allergen challenge but significantly reduced the number of neutrophils in BAL derived from treated patients compared with placebo (Evans et al., 1996).

7. Inflammatory Bowel Disease. Colonic mucosa derived from patients with inflammatory bowel disease (IBD) exhibit increased mRNA levels of LT-forming enzymes and enhanced $\mathrm{LTB}_{4}$ production (Sharon and Stenson, 1984; Jupp et al., 2007). However, studies performed with ex vivo stimulated human colonic mucosa have revealed that lower amounts of $\mathrm{LTB}_{4}$ are released compared with other arachidonic acid metabolites (Zijlstra et al., 1992, 1993). For example, 15-HETE is the dominant product formed after addition of exogenous arachidonic acid to human biopsy specimens obtained at coloscopy (Zijlstra et al., 1992, 1993). Furthermore, $\mathrm{HHT}$, which may act as a high-affinity $\mathrm{BLT}_{2} \mathrm{R}$ ligand (see section II.A and Fig. 1), is produced at 5- to 6-fold higher concentration compared with $\mathrm{LTB}_{4}$ (Zijlstra et al., 1992, 1993). Nevertheless, the exact role of these ligands and the two BLTR subtypes in IBD remains to be established. Whereas different BLTR antagonists inhibit colonic inflammation and neutrophil infiltration in animal models of IBD (Fretland et al., 1990, 1991), $\mathrm{BLT}_{2} \mathrm{R}$-deficient mice exhibit exacerbated colonic inflammation (Iizuka et al., 2010).

8. Cancer. $\mathrm{LTB}_{4}$ levels are increased in different human cancer tissues, such as prostate (Larré et al., 2008) and oral (el-Hakim et al., 1990) cancers. Furthermore, immunohistochemical analysis has revealed expression of BLT receptors in human pancreatic and colon cancers (Hennig et al., 2002; Ihara et al., 2007). As outlined above, $\mathrm{LTB}_{4}$ signals through pathways associated with cell proliferation, such as MAPK, ERK, and PI3K, which has also been implicated in tumor growth (Tong et al., 2005; Ihara et al., 2007). In support of the latter suggestion, in vitro studies have shown that $\mathrm{LTB}_{4}$ promotes and that $\mathrm{BLT}_{1} \mathrm{R}$ antagonists inhibit the proliferation of cultured human cancer cell lines (Earashi et al., 1995; Bortuzzo et al., 1996; Ihara et al., 2007). In addition to direct effects on tumor cells, the $\mathrm{BLT}_{2}$ receptor-associated angiogenesis discussed above (see section II.A) (Kim et al., 2009) may potentially also be involved in $\mathrm{LTB}_{4}$-induced cancer growth (Wang and Dubois, 2010a).

Administration of the $\mathrm{BLT}_{1} \mathrm{R}$ antagonist LY293111 after transplantation of human cancer cells to athymic mice resulted in reduced tumor growth and reduced incidence of metastases (Hennig et al., 2004, 2005). However, results from clinical trials using the latter antagonist in combination with gemcitabine did not improve the prognosis for patients with either pancreatic or lung cancer (Wang and Dubois, 2010a).

\section{G. Other Receptors Involved in BLT Receptor Signaling}

1. Peroxisome Proliferator-Activated Receptors. Peroxisome proliferator-activated receptors (PPARs) are li- 
gand-activated transcription factors that belong to the nuclear hormone receptor superfamily (Michalik et al., 2006). PPARs activate gene transcription associated with metabolism and inflammation, in response to synthetic PPAR ligands developed for the treatment of diabetes and dyslipidemia (Michalik et al., 2006). In addition, $\mathrm{LTB}_{4}$ has been identified among the endogenous ligands for PPAR $\alpha$. In transfected HeLa cells, $\mathrm{LTB}_{4}$ activated $\mathrm{PPAR} \alpha$ reporter gene transcription in the micromolar range and bound a fusion protein containing the ligand-binding domain of PPAR $\alpha$, with a $K_{\mathrm{d}}$ of $90 \mathrm{nM}$ (Devchand et al., 1996). Subsequent studies using different methods have confirmed $\mathrm{LTB}_{4}$ binding to and activation of PPAR $\alpha$ (Krey et al., 1997; Lin et al., 1999; Narala et al., 2010), although negative results have also been reported (Forman et al., 1997). In addition to results obtained in vitro, a recent study has also demonstrated that mice lacking $\mathrm{LTB}_{4}$ biosynthesis through 5-LO knockout exhibited reduced PPAR $\alpha$ activation in response to LPS administration in vivo (Narala et al., 2010). The latter results therefore support the observation that endogenous intracellular $\mathrm{LTB}_{4}$ formation can reach sufficient concentrations for PPAR $\alpha$ activation under inflammatory conditions (Narala et al., 2010).

2. Vanilloid Transient Receptor Potential V1 Receptor. The TRPV1 receptor is a ligand-gated, nonselective cation channel, which belongs to a family of TRP channels present exclusively in small sensory neurons and associated with pain. Although capsaicin and other vanilloids are the classic agonists, endogenous ligands derived from lipoxygenase metabolism of arachidonic acid and anandamide have also been reported to activate TRPV1 receptors (Hwang et al., 2000; McHugh et al., 2006). In inside-out patches of cultured dorsal root ganglion, TRPV1 receptors are activated by lipoxygenase products such as $12(S)$-HpETE, $15(S)$-HpETE, 5(S)HETE, and $\mathrm{LTB}_{4}$, whereas $\mathrm{LTC}_{4}$ is without effect (Hwang et al., 2000). The $\mathrm{EC}_{50}$ for $\mathrm{LTB}_{4}$-induced TRPV1 activation was $11 \mu \mathrm{M}$, which was 10 -fold higher than that obtained for capsaicin in the latter study (Hwang et al., 2000) and substantially lower compared with $\mathrm{BLT}_{1} \mathrm{R}$-mediated $\mathrm{LTB}_{4}$-induced signaling. In line with these findings, $\mathrm{LTB}_{4}$ also increased $\left[\mathrm{Ca}^{2+}\right]_{\mathrm{i}}$ in TRPV1transfected $\mathrm{CHO}$ cells, albeit with significantly lower $E_{\text {max }}$ and a longer exposure time required to achieve maximum effects compared with capsaicin (McHugh et al., 2006). Indeed, very recently it has also been demonstrated in vivo that both peripheral and spinal administration of RvE1 or RvD1 in mice potently reduces inflammatory pain behaviors, without affecting basal pain perception. These actions are transduced through RvE1mediated inhibition of TRPV- and TNF- $\alpha$-induced excitatory postsynaptic current increases in spinal dorsal horn neurons (Xu et al., 2010).

\section{CysLT Receptors}

\section{A. CysLT Receptor Subtypes}

\section{From Cloning CysLT Receptors to Recent Molecular} Advances. The CysLTRs eluded gene cloning with conventional approaches for many years. Eventually, in 1999 two separate groups cloned the first CysLTR (Lynch et al., 1999; Sarau et al., 1999), and in the following year the second receptor subtype was cloned by three different groups (Heise et al., 2000; Nothacker et al., 2000; Takasaki et al., 2000). Hydrophobicity analysis of the deduced primary amino acid sequences demonstrated that both receptors possess seven TM helices organized in a serpentine topology, confirming early studies that provided evidence that cysteinyl LTs were acting through a GPCRs and confirmed pharmacological evidence for CysLTR heterogeneity based on the functional as well as on ligand-binding data (for review of early reports, see Capra, 2004; Capra et al., 2007; Evans, 2003). To date well documented results have been obtained, establishing the existence of two receptor subtypes referred to as $\mathrm{CysLT}_{1}$ and $\mathrm{CysLT}_{2}$ (see section III.G). This nomenclature was based on the observations that the CysLT $_{1}$ receptor was sensitive to inhibition by classic antagonists, whereas the effects mediated by the $\mathrm{CysLT}_{2}$ receptor were not inhibited by these antagonists (Labat et al., 1992; Brink et al., 2003). These receptors belong to the rhodopsin family of the GPCR gene superfamily and, in particular, to the purine receptor cluster (within the $\delta$ group) of phylogenetically related receptors. In addition to a number of orphan receptors, this group includes receptors that respond to purinergic or pyrimidinergic nucleotides, proteases, and PAF (Fredriksson et al., 2003; Kroeze et al., 2003). Unlike the monoamine or neuropeptide receptors, the receptors belonging to the purine cluster have no clear homologs in invertebrates, suggesting a relatively recent evolutionary origin (Adams et al., 2000). Human CysLT $\mathrm{T}_{1}$ and $\mathrm{CysLT}_{2}$ receptors share only $38 \%$ aa identity, with very low homology in the extreme carboxyl termini. Of interest, human CysLTRs have higher homology with the purinoceptor P2Y1/2/6 (32-30\% aa identity) or GPR17 (34-32\% aa identity) than with the other known LT receptors, the $\mathrm{BLT}_{1 / 2}$ receptors ( $\leq 27 \%$ aa identity).

The human $\mathrm{CysLT}_{1}$ gene/chromosome location has been mapped on the long arms of chromosome X (Xq13$\mathrm{Xq} 21$ ), a region lacking asthmatic disease markers (Lynch et al., 1999), whereas CysLT $_{2}$ on human chromosome 13 (13q14) (Heise et al., 2000; Takasaki et al., 2000) is near a marker that has been associated with atopic asthma (Cookson, 1999; Kimura et al., 1999).

a. CysLT $T_{1}$ Receptor. The open reading frame of the human CysLT 1 encodes a protein of 337 aa (NCBI Reference Sequence: NP_006630) with a calculated molecular mass of $38 \mathrm{kDa}$, observed to migrate at a molecular mass of approximately $42 \mathrm{kDa}$ as a monomeric form (Bautz et al., 2001; Figueroa et al., 2001; Ohd et al., 
2003; Hasegawa et al., 2010), although oligomers were often observed (see section III.G). However, other authors observed monomeric migration at different molecular masses, possibly reflecting differences in the cell type, protein/cell maturation, or experimental conditions: 30 to $36 \mathrm{kDa}$ (Sjöström et al., 2001, 2002) and 38 to $40 \mathrm{kDa}$ (Capra et al., 2004). Human $\mathrm{CysLT}_{1} \mathrm{R}$ possesses four potential $\mathrm{N}$-glycosylation sites, one in the extracellular N-tail, two in the second extracellular loop, and one in the third extracellular loop, besides many potential protein kinase $\mathrm{A}$ and $\mathrm{C}$ phosphorylation sites (see section III.D), mostly located in the third intracellular loop and carboxyl terminal (Lynch et al., 1999). The rank order of potency for the cysteinyl LTs is $\mathrm{LTD}_{4}>\mathrm{LTC}_{4}$ when tested in different functional assays, such as stimulation of calcium-activated chloride conductance in Xenopus laevis oocytes or fluorescence calcium imaging in COS-7, HEK-293, or CHO human $\mathrm{CysLT}_{1}$-transfected cells. $\mathrm{LTE}_{4}$ is the less potent agonist acting as a partial agonist. As expected, the $\mathrm{LTD}_{4}$ functional response is potently inhibited by the selective CysLT $_{1} \mathrm{R}$ antagonists, 3-[[3-[2-(7-chloroquinolin-2yl)vinyl]phenyl]-(2-dimethylcarbamoylethylsulfanyl) methylsulfanyl] propionic acid (MK-571), zafirlukast, pranlukast, montelukast, and pobilukast (Brink et al., 2003; Evans, 2003; Capra, 2004). Saturation radioligand binding experiments on membranes from COS-7 cells expressing the human $\mathrm{CysLT}_{1} \mathrm{R}$ and using $\left[{ }^{3} \mathrm{H}\right] \mathrm{LTD}_{4}$ as a labeled ligand demonstrated a single $K_{\mathrm{d}}$ of $0.3 \mathrm{nM}$ (Lynch et al., 1999) or high- and low-affinity binding sites with $K_{\mathrm{d}}$ values of 0.06 and $6.2 \mathrm{nM}$ (Capra et al., 2005). $B_{\max }$ has been reported to be approximately 50 $\mathrm{fmol} / \mathrm{mg}$ membrane protein in both reports.

The genomic organization of the human CYSLTR1 gene has been obtained by 5 ' - and 3 '-rapid amplification of cDNA ends method, modified to ensure the amplification of only full-length transcripts (Woszczek et al., 2005). The gene consists of five exons that are variably spliced and a single promoter region TATA-less with multiple transcription start sites. Four different CysLT $_{1} R$ transcripts were identified with a dominant and wide expression of the transcript I, containing exons 1,4 , and 5 , with the strongest presence in blood leukocytes, spleen, thymus, lung, and heart (Woszczek et al., 2005). Successive articles reported that CYSLTR1 contains three exons with the entire open reading frame located in exon 3 and that five splice variants were detected in human leukocytes (Zhang et al., 2006), whereas in human airway SMCs and peripheral blood mononuclear cells (MNCs) three transcripts and, possibly, two putative promoters were found (Duroudier et al., 2007). In summary, multiple splice variants of $\mathrm{CysLT}_{1} \mathrm{R}$ exist, and the transcript expression patterns seem to differ from tissues and cell types. At present, the role of alternative splice variants of CysLT $_{1} R$ is not known. Woszczek et al. (2005) also reported in their study that the promoter region contains several binding sites for transcription factors, such as activator protein-1
(AP-1) and GATA and that CysLT $_{1} \mathrm{R}$ expression is functionally regulated at the transcriptional level by IL-4 through a signal transducer and activator of transcription (STAT)-6 response element localized to the proximal promoter region, confirming former studies by Thivierge et al. (2001) (see section III.B).

b. CysLT $T_{2}$ Receptor. The open reading frame of human CysLT $_{2} \mathrm{R}$ encodes a protein of 346 aa (NCBI Reference Sequence: NP_065110), which seems to migrate at a molecular mass of $58 \mathrm{kDa}$ in basophil lysates (Gauvreau et al., 2005) and approximately $50 \mathrm{kDa}$ in platelet (Hasegawa et al., 2010) or at approximately 40 to $42 \mathrm{kDa}$ in HUVECs and COS-7 cells transfected with human CysLT $_{2}$ cDNA (Carnini et al., 2011). Human CysLT ${ }_{2} \mathrm{R}$ possesses four potential N-glycosylation sites, three of which in the extracellular N-tail, as well as many potential protein kinase $\mathrm{A}$ and $\mathrm{C}$ phosphorylation sites mostly located in the third intracellular loop and carboxyl terminal (Heise et al., 2000). $\mathrm{Ca}^{2+}$ mobilization assay in HUVECs confirmed early observation in recombinant systems that $\mathrm{LTD}_{4}$ and $\mathrm{LTC}_{4}$ are equipotent agonists with an identical $\mathrm{EC}_{50}$ of $\sim 35 \mathrm{nM}$, and lack of sensitivity of the response to the classic $\mathrm{CysLT}_{1} \mathrm{R}$ antagonists, montelukast, zafirlukast, and pranlukast, whereas $\mathrm{LTE}_{4}$ and 6(R)-(4-carboxyphenylthio)-5(S)-hydroxy-7(E),9(E), $11(Z), 14(Z)$-eicosatetraenoic acid (BAY U9773 behave as partial agonists (Lötzer et al., 2003). Equilibrium binding studies in intact HUVECs using $\left[{ }^{3} \mathrm{H}\right] \mathrm{LTC}_{4}$ as labeled ligand revealed the presence of a high-affinity binding site with $K_{\mathrm{d}}$ of $29 \mathrm{pM}$ and $B_{\max }$ of $32 \mathrm{fmol} / 10^{8}$ cells (Carnini et al., 2011). In $\mathrm{CysLT}_{2}$-transfected COS-7 cell membranes high- and low-affinity binding sites with $K_{\mathrm{d}}$ values of 0.4 and $50 \mathrm{nM}$ or a single site of $4.8 \mathrm{nM}$ were obtained in two independent saturation experiments using $\left[{ }^{3} \mathrm{H}\right] \mathrm{LTD}_{4}$ as labeled ligand (Heise et al., 2000).

The genomic organization of the human CYSLTR2 has also been published recently. Similarly to CYSLTR1, the gene has a TATA-less promoter with multiple transcription start sites. Six variably spliced exons have been identified and eight different $\mathrm{CysLT}_{2} \mathrm{R}$ transcripts were also identified in endothelial and monocytic cells (Woszczek et al., 2007). It is noteworthy that IFN- $\gamma$ increased CysLT $_{2} \mathrm{R}$ mRNA expression and calcium signaling in ECs. However, there were no significant changes in gene reporter and mRNA $t_{1 / 2}$ assays in response to this cytokine, suggesting transcriptional control of CysLT $_{2} \mathrm{R}$ mRNA up-regulation by IFN- $\gamma$ response motifs localized outside of the cloned $\mathrm{CysLT}_{2} \mathrm{R}$ promoter region. Stimulation of ECs by cysteinyl LTs induced mRNA and protein expression of early growth response genes 1,2 , and 3 and cycloxygenase-2 (COX-2) (Woszczek et al., 2007).

2. Receptor Expression Patterns with Functional Significance. The pathophysiological role of cysteinyl LTs in asthma is well documented (Drazen, 2003; Holgate et al., 2003; Sampson et al., 2003; Arm, 2004; Capra et al., 2007; Hallstrand and Henderson, 2010), and results ob- 
tained from localization studies are consistent with the antibronchoconstrictive and anti-inflammatory activities of CysLT ${ }_{1}$ R antagonists (Kemp, 2003; Riccioni et al., 2004; Currie et al., 2005; Capra et al., 2006; Dahlén, 2006; Ducharme et al., 2006; Montuschi et al., 2007; del Giudice et al., 2009). However, the finding of CysLTR expression in other tissues will certainly encourage the discovery of new functions for cysteinyl LTs in other physiological and pathological conditions (Capra et al., 2007).

a. CysLT $T_{1}$ Receptor. Initial immunohistochemical analysis confirmed the presence of $\mathrm{CysLT}_{1} \mathrm{R}$ protein in a series of cells of particular relevance to asthma and atopy such as monocytes and eosinophils but also in pregranulocytic CD34 ${ }^{+}$cells and in subsets of B lymphocytes (Figueroa et al., 2001). Monocytes/macrophages isolated from peripheral blood MNCs express CysLT $_{1} R$ mRNA either in large excess compared with CysLT $_{2} R$ transcript levels (Lötzer et al., 2003) or, exclusively, such as in U937 (Capra et al., 2005), a human leukemic monocyte/macrophage cell line. This correlates with the finding that cysteinyl LTs contribute to inflammatory reactions by induction of $\mathrm{MCP}-1$ via the CCR2B receptor in THP-1 cells (Ichiyama et al., 2005; Woszczek et al., 2005; Hashimoto et al., 2009). This reported induction by MCP-1 was also observed in another monocytic leukemia cell line and was further supported by the enhanced activation of chemotactic activity observed in human monocytes (Woszczek et al., 2008a). Likewise, in THP-1 cells, montelukast and zafirlukast significantly down-regulated the chemotaxis induced by MCP-1 and p38 MAPK expression (Hung et al., 2006). These data suggest a dominant functional pathway for the interaction between cysteinyl LTs and MCP-1 in a variety of MNCs, including human monocytes. In animal models, inhibition of MCP-1 produces antiatherogenic effects in vivo in a rabbit carotid balloon injury model (Ge et al., 2009).

The expression of CysLTRs in eosinophils was not unexpected (Bandeira-Melo and Weller, 2003), because the contribution of cysteinyl LTs to their accumulation within asthmatic airways has been well documented (Gauvreau et al., 2001; Ohshima et al., 2002; Nagata and Saito, 2003; Saito et al., 2004). In a recent study, the CysLT $_{1} \mathrm{R}$ has been associated with cytokine transduction signals for the up-regulation of eosinophilopoiesis by IL-13 and eotaxin in murine bone marrow (Queto et al., 2010). These data support early reports demonstrating the inhibitory action of $\mathrm{CysLT}_{1} \mathrm{R}$ antagonists on eosinophil activation and migration (Virchow et al., 2001; Fregonese et al., 2002; Suzuki et al., 2003; Ueda et al., 2003; Saito et al., 2004; Nagata et al., 2005), as well as on adhesion (Fregonese et al., 2002; Nagata et al., 2002; Kushiya et al., 2006; Meliton et al., 2007; Profita et al., 2008). There is considerable information available to demonstrate the ability of CysLTR antagonists to reduce airway eosinophilia and eosinophil cationic pro- tein (ECP) in animals (Underwood et al., 1996; Ihaku et al., 1999) and in humans (Pizzichini et al., 1999; Obase et al., 2002; Steinke et al., 2003; Strauch et al., 2003; Laitinen et al., 2005; Kopriva et al., 2006).

CysLT $_{1} R_{s}$ are also found to be expressed in B lymphocytes and CD34 ${ }^{+}$hematopoietic progenitor cells (Figueroa et al., 2001). Indeed, early observations demonstrated that $\mathrm{LTD}_{4}$-stimulate chemotaxis and transendothelial migration of CD $34^{+}$hematopoietic progenitor cells (Bautz et al., 2001; Mohle et al., 2003) as well as their proliferation (Braccioni et al., 2002; Parameswaran et al., 2004; Boehmler et al., 2009) and that these activities were suppressed by different CysLT $T_{1}$ receptor antagonists. Altogether these data indicate a physiological role for cysteinyl LTs as autocrine regulators of hematopoiesis corroborated by the expression of $\mathrm{LTC}_{4} \mathrm{~S}$ in immature myeloid cells (Tornhamre et al., 2003).

Basophils have also been shown to accumulate in the airways of subjects with atopic asthma (Gauvreau et al., 2001), and these authors documented that human basophils express variable levels of functional $\mathrm{CysLT}_{1} \mathrm{R}$. Of interest, they found that prolonged stimulation of cell cultures with $\mathrm{LTD}_{4}$ reduced the frequency of CD95 Fas receptor expression, an effect that, albeit modest, was reversed by the $\mathrm{CysLT}_{1} \mathrm{R}$ antagonist zafirlukast (Gauvreau et al., 2005). These results seem to suggest that the cysteinyl LT/CysLT ${ }_{1} \mathrm{R}$ system might have a direct effect on basophil accumulation in allergic tissues.

MCs are a primary source of cysteinyl LTs (Austen, 2005) after allergic or nonallergic stimulation and, accordingly to their classification as autacoids, CysLTRs are widely expressed in these cells (Mellor et al., 2001; Sjöström et al., 2002) and are responsible for their proliferation and activation (Jiang et al., 2006, 2009; Kaneko et al., 2009). In agreement with this finding, montelukast has been found to significantly reduce the number of MCs in the inflamed paws of mice with collagen-induced arthritis (Shiota et al., 2006).

The expression of both CysLT $_{1} R$ and CysLT $_{2} R$ has also been reported in human platelets by RT-PCR, Western blotting, and flow cytometry (Hasegawa et al., 2010). In this report, the authors demonstrate that cysteinyl LTs induced a release of RANTES and that this effect was inhibited by pranlukast, suggesting a novel role for platelets in allergic inflammation (Hasegawa et al., 2010).

In agreement with a role of cysteinyl LTs in asthma, CysLT $_{1} R_{s}$ are expressed by a variety of airway mucosal inflammatory cells and the numbers of inflammatory $\mathrm{CysLT}_{1} \mathrm{R}$-expressing cells (eosinophils, neutrophils, MCs, macrophages, and B lymphocytes but not T lymphocytes) significantly increase in subjects with stable asthma and patients hospitalized for asthma exacerbation compared with control subjects (Zhu et al., 2005). Of interest, a strong positive correlation exists between this observation and the augmented numbers of CD $45^{+}$progenitors (cells expressing the pan-leukocyte marker 
CD45 antigen) (Zhu et al., 2005). In addition, human fibroblasts have been shown to express both CysLT $_{1}$ (James et al., 2006; Vannella et al., 2007) and CysLT 2 receptors (Vannella et al., 2007). These cells are also known to produce cysteinyl LTs, suggesting a regulation via an autocrine/paracrine secretion of these lipid mediators. Indeed, exogenous administration of $\mathrm{LTD}_{4}$ but not of $\mathrm{LTC}_{4}$ was also able to induce proliferation of both murine and human fibrocytes, and $\mathrm{CysLT}_{1} \mathrm{R}$ antagonists blocked this mitogenic effect (Vannella et al., 2007). In contrast, there are two reports that evaluated the contribution of cysteinyl LTs to fibroblast-mediated fibrosis and remodeling in chronic hyperplastic eosinophilic sinusitis (Steinke et al., 2004) or in healthy subjects (Yoshisue et al., 2007) and found that fibroblasts did not substantially express CysLT $_{1} R$ or CysLT $_{2} R$, even after priming with IL-4 or IL-13 (Steinke et al., 2004). However, cysteinyl LTs have a broader capability to synergize with epidermal growth factor receptor (EGFR) to increased thymidine incorporation and cell proliferation, effects that, in agreement with the absence of CysLTRs, are not inhibited by specific CysLT $_{1} R$ antagonists (MK571 and montelukast) or by the dual antagonist BAY U9773, pointing to a different mechanism/receptor (Yoshisue et al., 2007).

In human nasal mucosa, increased expression of $\mathrm{CysLT}_{1} \mathrm{R}$ has been found both at gene and protein levels in blood vessels and in the interstitial vascular ECs, as well as in eosinophils, MCs, macrophages, and neutrophils (Shirasaki et al., 2002, 2006) and in nasal polyps (Chao et al., 2006; Pérez-Novo et al., 2006). Because subjects with aspirin-induced asthma (AIA) have greater airway hyperresponsiveness to the effects of inhaled cysteinyl LTs than those with aspirin-tolerant asthma (ATA) (Arm et al., 1989), Sousa and colleagues (Sousa et al., 2002; Corrigan et al., 2005) hypothesize that this could be due to the elevated expression of $\mathrm{CysLT}_{1} \mathrm{R}$ on inflammatory cells. Their study on nasal biopsy specimens from patients with chronic rhinosinusitis and nasal polyps revealed that aspirin-sensitive rhinosinusitis is indeed characterized by increased numbers of nasal inflammatory leukocytes expressing the $\mathrm{CysLT}_{1} \mathrm{R}$. These results have been confirmed (Ozen et al., 2007) and are consistent with two other reports in which the augmented receptor expression was observed for the majority of eosinophils and subsets of MNCs obtained by nasal lavage (Figueroa et al., 2003) or in sputum after allergen challenges in patients with seasonal allergic rhinitis (AR) (Boulay et al., 2010). Finally, CysLT $_{1}$ but not CysLT $_{2}$ mRNA has been demonstrated in nasal C-fiber neurons, where $\mathrm{LTD}_{4}$ directly increased the excitability of capsaicin-sensitive guinea pig nasal trigeminal neurons. These observations suggest a novel mechanism for the actions of cysteinyl LTs and offer an explanation for the observed beneficial use of $\mathrm{CysLT}_{1} \mathrm{R}$ antagonists in treating nasal allergen-induced neuronal symptoms (Taylor-Clark et al., 2008).
RT-PCR has allowed the demonstration that human saphenous veins express both a functional CysLT $_{1} R$, which mediates contractile effects of cysteinyl LTs, but also a CysLT $\mathrm{T}_{2} \mathrm{R}$, which, on the contrary, does not seem to be implicated in contraction (Mechiche et al., 2004) and whose functional role remains to be determined (Allen et al., 1992). Human pulmonary veins also express both receptors and although activation of the former induced the release of a contractile factor only partially blocked by CysLT $_{1} R$ antagonists, the activation of the CysLT $_{2} R$ released nitric oxide (NO) (Ortiz et al., 1995). In addition, human pulmonary artery vascular SMCs seem to express at least two different receptors, a CysLT ${ }_{1} R$ and a novel CysLTR subtype, both responsible for vasoconstriction (Walch et al., 2002). Thus, the mechanical effects of $\mathrm{LTD}_{4}$ on human pulmonary vasculature are complex and involve both direct and indirect mechanisms mediated via at least two, and possibly more, types of cysteinyl LT receptors (Walch et al., 2000).

In the gastrointestinal system, CysLT $_{1} \mathrm{R}$ expression has been documented in the small intestine and colon (Lynch et al., 1999; Sarau et al., 1999). Subsequently, the presence of the CysLT $_{1} R$ subtype was reported in a nontransformed epithelial intestinal cell line (Ohd et al., 2003), corroborating previous findings for $\mathrm{LTD}_{4}$-induced signaling in these cells (Grönroos et al., 1995; Thodeti et al., 2000, 2001). In addition, $\mathrm{CysLT}_{1} \mathrm{R}$ functions were characterized in colorectal carcinoma cells (Ohd et al., 2003), and low expression of CysLT $_{1} R$ and high expression of $\mathrm{CysLT}_{2} \mathrm{R}$ correlated with high differentiation and good prognosis (Magnusson et al., 2007, 2010). The same results have been also extended to breast cancer cells and patient survival (Magnusson et al., 2011).

b. CysLT $T_{2}$ Receptor. Localization studies have identified a distinctive expression pattern for human $\operatorname{CysLT}_{2} \mathrm{R}$, despite some overlapping with $\mathrm{CysLT}_{1} \mathrm{R}$. Indeed, expression in heart, particularly in Purkinje fiber cells, myocytes, and fibroblasts derived from atrium and ventricle, brain, and adrenal glands is specific to $\mathrm{CysLT}_{2} \mathrm{R}$ (Heise et al., 2000; Nothacker et al., 2000; Takasaki et al., 2000).

Several authors indicated that HUVECs almost exclusively express CysLT $_{2} \mathrm{R}$ (Mita et al., 2001b; Lötzer et al., 2003), which is responsible for the calcium mobilization and contraction evoked in these cells by cysteinyl LTs, as well as by the selective CysLT $_{2} \mathrm{R}$ agonist BAY U9773 (Sjöström et al., 2003; Carnini et al., 2011), for which activation results in a proinflammatory EC phenotype (Uzonyi et al., 2006). ECs are strategically located at the interface with blood circulation where they become exposed to neutrophil- and platelet-derived LTs, a setting that has potential implications for cardiovascular diseases (CVDs). Moreover, previous studies demonstrated that cysteinyl LTs can trigger several functional responses in ECs, such as PAF accumulation and neutrophil adhesion (McIntyre et al., 1986) and secretion of von Willebrand factor and of P-selectin surface expression (Datta et al., 1995). In light of these expression data, the 
responses data can be attributed to the $\mathrm{CysLT}_{2} \mathrm{R}$ and, accordingly, are not inhibited by selective CysLT $_{1} R$ antagonists (Pedersen et al., 1997).

Kamohara et al. (2001) were the first to report the presence of a functional $\mathrm{CysLT}_{2} \mathrm{R}$ on human coronary artery SMCs. These authors demonstrated that $\mathrm{LTC}_{4}$ enhanced $\left[\mathrm{Ca}^{2+}\right]_{\mathrm{i}}$, an effect that was not blocked by CysLT $_{1} R$ antagonists but blocked by the calcium channel blocker nicardipine. Further significance of cysteinyl LTs involvement in atherosclerosis arises from the observation that cysteinyl LTs induce contractions of human atherosclerotic coronary arteries, whereas nonatherosclerotic arteries are unresponsive (Allen et al., 1998). Taken together, these results suggest that the activation of $\mathrm{CysLT}_{2} \mathrm{R}$ can induce profound effects in cardiac as well as in hemodynamic and microcirculatory pathophysiology and that this receptor subtype represents an interesting pharmacological target in the future for CVDs.

Although little expression for $\mathrm{CysLT}_{1} \mathrm{R}$ has been reported in brain (Lynch et al., 1999; Sarau et al., 1999), apparent localization has been documented by immunohistochemical analysis in human brains with traumatic injury or tumors (Zhang et al., 2004). In contrast, $\mathrm{CysLT}_{2} \mathrm{R}$ mRNA is highly expressed in the central nervous system, with particular concentration in hypothalamus, thalamus, putamen, pituitary, and medulla (Heise et al., 2000) and in small, but not large, vessels in mouse brain (Moos et al., 2008). A number of articles have reported the involvement of either $\mathrm{CysLT}_{1}$ (Fang et al., 2006) or $\mathrm{CysLT}_{2}$ (Fang et al., 2007) or both receptors (Sheng et al., 2006; Wang et al., 2006; Huang et al., 2008) in the inflammatory process subsequent to brain vascular insults (vascular ischemia or oxygen deprivation). Spatiotemporal expression of $\mathrm{CysLT}_{2} \mathrm{R}$ mRNA in rat brain was observed after focal cerebral ischemia induced by middle cerebral artery occlusion, suggesting that $\operatorname{CysLT}_{2} \mathrm{R}$ may be related to the acute neuronal injury and late astrocyte proliferation in the ischemic brain (Fang et al., 2007).

In the immune system, moderate expression of $\mathrm{CysLT}_{2}$ mRNA was seen in spleen, lymph nodes, and peripheral blood leukocytes (Heise et al., 2000). A comparative study on the levels of CysLTR subtype expression in human peripheral blood leukocytes (eosinophils, neutrophils, monocytes, and T lymphocytes) indicated a significantly high expression for CysLT $_{2}$ mRNA in eosinophils (Mita et al., 2001b), suggesting unidentified roles for this receptor in these cells. Mellor et al. (2003) reported that human MCs constitutively express the $\mathrm{CysLT}_{2} \mathrm{R}$, whose proposed function seems to be the production of IL-8. CysLT 2 R expression was also reported in basophils (Gauvreau et al., 2005), but a functional role for this protein was not identified, despite flow cytometric analysis, which revealed an expression level equivalent to that of the $\operatorname{CysLT}_{1} \mathrm{R}$. No CysLT $_{2} \mathrm{R}$ expression was found in either undifferentiated or differentiated promyelocytic HL-60 and U937 cells (Nothacker et al., 2000; Capra et al., 2005).

CysLT $_{2} \mathrm{R}$ was expressed in the majority of eosinophils and in subsets of MCs and MNCs, but not in neutrophils, obtained by nasal lavage of patients with seasonal AR (Figueroa et al., 2003). Sousa et al. (2002) and Corrigan et al. (2005) extended a previous study on $\mathrm{CysLT}_{1} \mathrm{R}$ expression in the nasal mucosa of subjects with chronic rhinosinusitis, both AIA and ATA, and found that the distribution of $\mathrm{CysLT}_{2} \mathrm{R}$ differs from that of $\mathrm{CysLT}_{1}$, with a predominance of $\mathrm{CysLT}_{2} \mathrm{R}$ on glands and epithelium. They also showed that nasal mucosal inflammatory leukocytes showed no evidence of up-regulation of CysLT $_{2} \mathrm{R}$ in subjects with rhinosinusitis, whether AIA or ATA, compared with control subjects (Corrigan et al., 2005). However, $\mathrm{CysLT}_{2} \mathrm{R}$ up-regulation has been demonstrated in nasal polyp tissue and expression was correlated with eosinophilic inflammation (Pérez-Novo et al., 2006). In human lung, the CysLT ${ }_{2}$ mRNA signal was high in interstitial macrophages and weak in SMCs (Heise et al., 2000), in which, on the contrary, CysLT mRNA expression was elevated. Of interest, RT-PCR revealed that A549 cells, a human lung adenocarcinomaderived line with alveolar epithelial cell properties, express mRNA for $\mathrm{CysLT}_{2} \mathrm{R}$ but not $\mathrm{CysLT}_{1} \mathrm{R}$ (Sloniewsky et al., 2004).

The adrenal gland may represent a novel tissue for future studies on cysteinyl LT functions and $\mathrm{CysLT}_{2} \mathrm{R}$ role in modulating the endocrine system because strong expression was detected in medullary pheochromocytes (Lynch et al., 1999; Nothacker et al., 2000).

3. Localization of CysLT Receptors. The mechanism of action of cysteinyl LTs is thought to be primarily dependent on their specific plasma membrane receptors that, as discussed above, belong to the superfamily of GPCRs. However, a few reports indicate that CysLTR could also be localized to a nuclear compartment, suggesting major unanticipated roles for these receptors in cell signaling and function. Bandeira-Melo et al. (2002b) provided the first evidence for an intracrine CysLTR-induced signaling of eosinophil vesicular transport-mediated IL-4 secretion, which is PTX-sensitive. Furthermore, CysLT $_{1} R$ was found to be located in the outer nuclear membrane in colon cancer cells and may apparently be translocated into the nucleus after prolonged exposure to the agonist in nontransformed intestinal epithelial cells (Nielsen et al., 2005a). These authors also demonstrated that a nuclear localization sequence (localized in the $\mathrm{C}$ tail) is essential for the receptor translocation to the nucleus, as already demonstrated for other GPCRs (Boivin et al., 2008) as well as for other proteins that normally target the nucleus, such as 5-LO (Hanaka et al., 2002). Finally, $\mathrm{CysLT}_{1} \mathrm{R}$ expression in myofibroblasts derived from human heart valves exhibits a perinuclear pattern, and $\mathrm{LTC}_{4}$ increased nuclear $\left[\mathrm{Ca}^{2+}\right]$ in those cells (Nagy et al., 2011).

Because other lipid mediators have been demonstrated to exert biological functions including transcrip- 
tional regulation of COX-2 and inducible NO synthase through their nuclear GPCRs (Marrache et al., 2005), one can speculate that the roles for nuclear receptors may be different from those on plasma membrane and that intracellular GPCRs may constitute a distinctive mode of action for gene regulation (Boivin et al., 2008). A recent report has shown that $\mathrm{CysLT}_{1} \mathrm{R}$ and $\mathrm{CysLT}_{2} \mathrm{R}$, but also the purinergic $\mathrm{P}_{2} \mathrm{Y}_{12}$ receptor, are expressed on eosinophil granule membranes and that cysteinyl LTs stimulated isolated eosinophil granules to secrete ECP. Of interest, although montelukast inhibited ECP release, the $\mathrm{P}_{2} \mathrm{Y}_{12}$ receptor antagonist 2,2-dimethyl-propionic acid 3-(2-chloro-6-methylaminopurin-9-yl)-2-(2,2dimethyl-propionyloxymethyl)-propyl ester (MRS 2395) also inhibited cysteinyl LT-induced ECP release, adding another piece of evidence to the CysLT-P2Y receptor interaction puzzle (Neves et al., 2010) (see sections III.D and III.H).

\section{B. CysLT Receptor Expression and Immunoregulation}

Human CysLT $_{1} \mathrm{R}$ expression was up-regulated through augmented transcriptional activity by priming cells with Th2-like cytokines (i.e., IL-5 in eosinophil-differentiated HL-60 cells) (Thivierge et al., 2000) and IL-4 and IL-13 in human monocytes and monocyte-derived macrophages (Thivierge et al., 2001; Shirasaki et al., 2007). The regulation of human CysLT $_{1} \mathrm{R}$ expression by IL-13 has also been reported in the literature for lung fibroblasts (Chibana et al., 2003) and airway SMCs together with TGF- $\beta$ and IFN- $\gamma$, whereas, surprisingly, IL-4 was found to be ineffective (Espinosa et al., 2003). In the latter cells, up-regulation of $\mathrm{CysLT}_{1} \mathrm{R}$ by IFN- $\gamma$ has been demonstrated, and this correlates with increases in $\mathrm{LTD}_{4}$-induced responses (Amrani et al., 2001). HUVECs express $\operatorname{CysLT}_{1} R$ at a very low level (Sjöström et al., 2001), unless they are subjected to prolonged treatment with IL-1 $\beta$ (Gronert et al., 2001). The up-regulation by IL- $1 \beta$ and IFN- $\gamma$ seems intriguing because these cytokines are generally considered to be counterregulatory, inhibiting both the production and activities of the proallergic Th2-like cytokines. Use of IFN- $\gamma$ is indeed one of the theoretical approaches to obtain IgE immunomodulation in asthma, albeit adverse effects suggest limited use (Stokes and Casale, 2004). However, there are also investigators who suggest that IFN- $\gamma$ is likely to be proinflammatory in many aspects of chronic allergic inflammation, evolving our understanding of classic Th2 diseases (Liu, 2000).

Priming human MCs with IL-4 did not result in the up-regulation of $\mathrm{CysLT}_{1} \mathrm{R}$, either at the transcript or the protein level, but rather resulted in the appearance of an apparently new CysLTR also activated by the pyrimidinergic ligand UDP (Mellor et al., 2001). In a subsequent report, the same authors also demonstrated that human MCs primed with IL-4 responded to UDP, $\mathrm{LTC}_{4}$, and $\mathrm{LTD}_{4}$ by producing IL-5, TNF- $\alpha$, and especially large quantities of macrophage inflammatory protein (MIP)- $1 \beta$ that were inhibited by the $\mathrm{CysLT}_{1} \mathrm{R}$ antagonist
MK-571. Nevertheless, human MCs constitutively also express the $\mathrm{CysLT}_{2} \mathrm{R}$, and IL-4 consistently up-regulated surface expression on a fraction of cells (Mellor et al., 2003). Whether IL-4 priming up-regulates a known CysLTR or induces the expression of a new receptor subtype (see section III.G) remains to be established.

A 2- to 3-fold enhancement of $\mathrm{CysLT}_{1} \mathrm{R}$ expression was observed in B lymphocytes after exposure to a combination of activating anti-CD40 antibody and IL-4. This enhancement, in turn, induced an increase in responsiveness to $\mathrm{LTD}_{4}$ in terms of $\mathrm{Ca}^{2+}$ flux and up-regulation of IgE and IgG production, which was totally prevented by the selective $\mathrm{CysLT}_{1} \mathrm{R}$ antagonist montelukast (Lamoureux et al., 2006). Therefore, Early et al. (2007) showed that IL-4, but not IL-13, was able to significantly induce mRNA and protein concentrations for $\mathrm{CysLT}_{1} \mathrm{R}$ from $\mathrm{T}$ and B lymphocytes.

CysLTRs can also be immunoregulated by cytokines that play an essential role in down-modulating adaptive and innate immune responses. IL-10, for example, down-regulated mRNA of $\mathrm{CysLT}_{1}$ and $\mathrm{CysLT}_{2}$ receptors in a time- and concentration-dependent fashion. In addition, cysteinyl LT-induced activation and chemotaxis of human monocytes and monocyte-derived immature DCs, measured by cytosolic $\mathrm{Ca}^{2+}$ flux and immediateearly gene expression, was potently decreased by IL-10 and by the CysLT 1 antagonist MK-571 (Woszczek et al., 2008b). Of interest, maturation of DCs with LPS (Thivierge et al., 2006), a classic Toll-like receptor 4 agonist, or zymosan (Thivierge et al., 2009), a Toll-like receptor 2 agonist, down-regulated $\mathrm{CysLT}_{1} \mathrm{R}$ mRNA levels and protein expression and reduced functional responsiveness to $\mathrm{LTD}_{4}$. Indeed, the effect of zymosan was, at least partially, dependent on endogenous production of $\mathrm{PGE}_{2}$ and IL-10 (Thivierge et al., 2009). Therefore, montelukast prevents the decrease of IL-10 and inhibits NF- $\kappa$ B activation in inflammatory airway of "asthmatic" guinea pigs (Wu et al., 2006).

Transcript levels of $\mathrm{CysLT}_{2} \mathrm{R}$ seem to be up-regulated by the Th2-like cytokine IL-4 in HUVECs (Lötzer et al., 2003) but suppressed by Th1-like (proinflammatory) cytokines, such as TNF- $\alpha$ (Lötzer et al., 2003; Sjöström et al., 2003), LPS, or IL-1 $\beta$ (Sjöström et al., 2003) in a rapid and partially reversible manner. However, IFN- $\gamma$ has been found to induce CysLT $_{2} R$ expression and to enhance the responsiveness to cysteinyl LTs of human ECs (Woszczek et al., 2007), as well as of monocytes, T cells, and B lymphocytes (Early et al., 2007). These data confirm early observations by Fujii et al. (2005), who postulated that $\mathrm{CysLT}_{2} \mathrm{R}$ might modulate exacerbations of asthma, as they observed that $\mathrm{CysLT}_{2} \mathrm{R}$ expression on eosinophils was up-regulated by IFN- $\gamma$ and increased during asthma exacerbation, especially in nonatopic subjects. In addition, another proinflammatory cytokine, IL-18, has also been recently postulated to upregulate $\mathrm{Cys}_{2} \mathrm{R}$ expression in HUVECs at the early 
stage of administration, accelerating cell apoptosis (Zhou et al., 2009).

Besides in HUVECs, CysLT $_{2} \mathrm{R}$ expression was also significantly increased in monocytes and eosinophils after IL-4 priming (Early et al., 2007) or IL-13 treatment (Shirasaki et al., 2007). At variance with data reported previously for CysLT $_{1} R$, CysLT $_{2} R$ expression increased after DC maturation induced by LPS, suggesting that these cells may differentially respond to cysteinyl LTs, depending on their maturational stimuli (Thivierge et al., 2006).

These observations suggest that the expression of the CysLTRs may be functionally up-regulated, mainly by Th2-like cytokines (consistent with the classic view of the pathobiological mechanisms of asthma and other allergic diseases), but also by a classic Th1 cytokine (i.e., IFN- $\gamma$ ) at least in some cells (Table 3). This finding raises the possibility that constitutive and inducible receptors may or may not behave in a similar way, prompt- ing a detailed pharmacological examination of receptors expressed before and after priming.

\section{Intracellular Signaling Pathways and Second-Messenger Systems}

1. CysLT $T_{1}$ Receptor. In consideration of the crucial bronchoconstrictor activity of cysteinyl LTs (Hallstrand and Henderson, 2010) and their role in asthma (Capra et al., 2007), intracellular $\mathrm{Ca}^{2+}$ mobilization and phosphatidylinositol (PI) metabolism were the obvious signal transduction systems to investigate (for a review of early reports, see Brink et al., 2003; Rovati and Capra, 2007). Upon cloning, $\mathrm{LTD}_{4}$-induced functional responses in oocytes (Lynch et al., 1999) or HEK-293 cells (Sarau et al., 1999) indicated that, at least in these systems, CysLT $_{1} R$ is very weakly, if not at all, coupled to a PTXinsensitive $\mathrm{G}$ protein $\left(\mathrm{G}_{\mathrm{q} / 11}\right)$.

However, an initial study demonstrated in circulating MNCs that cysteinyl LTs modulate $\mathrm{Ca}^{2+}$ responses

TABLE 3

Immunoregulation of human CysLTR expression

\begin{tabular}{|c|c|c|c|c|}
\hline Receptor & Cytokine & Cell & Effect $^{a}$ & Reference \\
\hline \multirow[t]{17}{*}{ CysLT $_{1}$} & IL-4 & $\begin{array}{l}\text { Monocytes and monocytes-derived } \\
\text { macrophages }\end{array}$ & $\uparrow$ mRNA; $\uparrow ;$ protein; $\uparrow\left[\mathrm{Ca}^{2+}\right]_{\mathrm{i}}$ & Thivierge et al., 2001 \\
\hline & & & $\uparrow \mathrm{mRNA}$ & $\begin{array}{l}\text { Woszczek et al., 2005; } \\
\quad \text { Shirasaki et al., } 2007\end{array}$ \\
\hline & + Anti-CD40 antibody & B lymphocytes & $\uparrow$ mRNA; $\uparrow$ protein & Early et al., 2007 \\
\hline & & & $\uparrow\left[\mathrm{Ca}^{2+}\right]_{\mathrm{i}} ; \uparrow \mathrm{IgE} / \mathrm{IgG}$ production & Lamoureux et al., 2006 \\
\hline & & T lymphocytes & $\uparrow$ mRNA; $\uparrow$ protein & Early et al., 2007 \\
\hline & IL-5 & Eosinophil-differentiated HL60 & $\uparrow$ mRNA; $\uparrow$ protein; $\uparrow\left[\mathrm{Ca}^{2+}\right]_{\mathrm{i}}$ & Thivierge et al., 2000 \\
\hline & IL-13 & $\begin{array}{l}\text { Monocytes and monocyte-derived } \\
\text { macrophages }\end{array}$ & $\uparrow$ mRNA; $\uparrow$ protein; $\uparrow\left[\mathrm{Ca}^{2+}\right]_{\mathrm{i}}$ & Thivierge et al., 2001 \\
\hline & & & $\uparrow \mathrm{mRNA}$ & $\begin{array}{l}\text { Thivierge et al., 2001; } \\
\quad \text { Shirasaki et al., } 2007\end{array}$ \\
\hline & & Lung fibroblasts & $\begin{array}{l}\uparrow \operatorname{mRNA} ; \uparrow, \text { protein; } \uparrow \text { eotaxin } \\
\text { production }\end{array}$ & Chibana et al., 2003 \\
\hline & & Airway SMCs & $\begin{array}{l}\text { No effect on mRNA; } \uparrow \text { protein; } \uparrow \text { cell } \\
\text { proliferation }\end{array}$ & Espinosa et al., 2003 \\
\hline & IFN- $\gamma$ & Airway SMCs & $\begin{array}{l}\uparrow \text { mRNA; } \uparrow \text { protein; } \uparrow \text { cell } \\
\text { stiffness }\end{array}$ & Amrani et al., 2001 \\
\hline & & Airway SMCs & $\begin{array}{l}\uparrow \text { mRNA; } \uparrow \text { protein; no effect on } \\
\text { cell proliferation }\end{array}$ & Espinosa et al., 2003 \\
\hline & IL-1 $\beta$ & HUVECs & $\uparrow \mathrm{mRNA}$ & Gronert et al., 2001 \\
\hline & TGF- $\beta$ & Airway SMCs & $\begin{array}{l}\text { No effect on mRNA; } \downarrow \text { protein; } \\
\downarrow \text { cell proliferation }\end{array}$ & Espinosa et al., 2003 \\
\hline & IL-10 (zymosan-induced) & $\begin{array}{l}\text { Monocytes and monocyte-derived } \\
\text { dendritic cells }\end{array}$ & $\downarrow$ mRNA; $\downarrow\left[\mathrm{Ca}^{2+}\right]_{\mathrm{I}}$ and chemotaxis & Woszczek et al., 2008b \\
\hline & & & $\begin{array}{l}\downarrow \underset{\text { mRNA; }}{\downarrow}\left[\mathrm{Ca}^{2+}\right]_{\mathrm{i}} \text { and } \\
\text { chemotaxis }\end{array}$ & Thivierge et al., 2009 \\
\hline & LPS & Monocyte-derived dendritic cell & $\underset{\text { chemotaxis }}{\downarrow \text { mRNA; }} \downarrow$ & Thivierge et al., 2006 \\
\hline \multirow[t]{13}{*}{ CysLT $_{2}$} & IL-4 & HUVECs & $\uparrow \mathrm{mRNA}$ & Lötzer et al., 2003 \\
\hline & & Mast cells & $\uparrow$ protein & Mellor et al., 2003 \\
\hline & & $\begin{array}{l}\text { B and T lymphocytes, monocytes, } \\
\text { eosinophils }\end{array}$ & $\uparrow$ mRNA; $\uparrow$ protein & Early et al., 2007 \\
\hline & IL-13 & Monocytes & $\uparrow \mathrm{mRNA}$ & Shirasaki et al., 2007 \\
\hline & IFN- $\gamma$ & Eosinophils (asthmatics) & $\uparrow$ mRNA; $\uparrow$ protein & Fujii et al., 2005 \\
\hline & & ECs & $\uparrow \operatorname{mRNA} ; \uparrow\left[\mathrm{Ca}^{2+}\right]_{\mathrm{i}}$ & Woszczek et al., 2007 \\
\hline & & Monocytes, T and B lymphocytes & $\uparrow$ mRNA; $\uparrow$ protein & Early et al., 2007 \\
\hline & IL-18 & HUVECs & $\uparrow$ mRNA; $\uparrow$ protein (first $2 \mathrm{~h}$ ) & Zhou et al., 2009 \\
\hline & TNF- $\alpha$ & HUVECs & $\downarrow$ mRNA & $\begin{array}{l}\text { Lötzer et al., 2003; Sjöström } \\
\text { et al., } 2003\end{array}$ \\
\hline & LPS & HUVECs & $\downarrow$ mRNA & Sjöström et al., 2003 \\
\hline & & Monocyte-derived dendritic cell & $\uparrow$ mRNA; $\uparrow$ protein & Thivierge et al., 2006 \\
\hline & IL-1 $\beta$ & HUVECs & $\downarrow$ mRNA & Sjöström et al., 2003 \\
\hline & IL-10 & $\begin{array}{l}\text { Monocytes and monocyte-derived } \\
\text { dendritic cells }\end{array}$ & $\downarrow$ mRNA & Woszczek et al., 2008b \\
\hline
\end{tabular}

${ }^{a} \uparrow$, up-regulation; $\downarrow$, down-regulation; specified are the effects (at mRNA, protein, and functional level). 
through a PTX-sensitive $\mathrm{G}$ protein $\left(\mathrm{G}_{\mathrm{i} / \mathrm{o}}\right)$ (Baud et al., 1987). In further reports, investigators have shown that the $\mathrm{Ca}^{2+}$ modulation may be through two distinct $\mathrm{G}$ proteins, one PTX-sensitive and one insensitive, in monocyte/macrophage U937 cells (Pollock and Creba, 1990; Capra et al., 2003) or in a human epithelial cell line (Sjölander et al., 1990; Adolfsson et al., 1996). Subsequently, $\mathrm{CysLT}_{1} \mathrm{R}$-dependent actin reorganization was shown to be coupled with a PTX-sensitive G protein and to Rho in an intestinal epithelial cell line (Grönroos et al., 1996; Massoumi and Sjölander, 1998; Massoumi et al., 2002) or in bronchial SMCs (Saegusa et al., 2001). Thus, these data confirm $\operatorname{CysLT}_{1} \mathrm{R}$ promiscuity in $\mathrm{G}$ protein coupling in constitutive systems, in good agreement with the finding that $\mathrm{LTD}_{4}$ activates distinct signaling pathways differently coupled to $G$ proteins in MNCs (Saussy et al., 1989; Skoglund and Claesson, 1991; Hoshino et al., 1998; Capra et al., 2003) or intestinal epithelial cells (Grönroos et al., 1998; Paruchuri and Sjölander, 2003; Nielsen et al., 2005b). Among these pathways several groups have demonstrated the activation of MAPK by CysLT ${ }_{1} \mathrm{R}$ in THP- 1 cells (Hoshino et al., 1998), renal mesangial cells (McMahon et al., 2000), intestinal epithelial cells (Paruchuri et al., 2002), monocyte/macrophage U937 cells (Capra et al., 2004), astrocytes (Ciccarelli et al., 2004), colon cancer cells (Parhamifar et al., 2005), MCs (Jiang et al., 2006), and airway SMCs (Ravasi et al., 2006). Cysteinyl LTs have been shown to induce proliferation in a variety of cells, such as human hematopoietic cell lines (Snyder et al., 1989; Braccioni et al., 2002; Parameswaran et al., 2004), airway epithelial cells (Leikauf et al., 1990), vascular (Porreca et al., 1995, 1996; Kaetsu et al., 2007) and airway SMCs (Cohen et al., 1995; Panettieri et al., 1998; Espinosa et al., 2003; Bossé et al., 2008), glomerular mesangial cells (Kelefiotis et al., 1995), intestinal epithelial cells (Paruchuri and Sjölander, 2003), astrocytes (Fang et al., 2006; Huang et al., 2008) and fibrocytes (Vannella et al., 2007).

There are many possible pathways through which GPCRs may induce ERK1/2 activation, one of which requires the transactivation of a growth factor receptor. Indeed, a first report suggested that $\mathrm{LTD}_{4}$ synergizes with the insulin growth factor axis to induce airway SMC proliferation (Cohen et al., 1995), involving proteolysis of airway SMC-produced inhibitory insulin growth factor-binding proteins by $\mathrm{LTD}_{4}$-induced MMP-1 (Rajah et al., 1996). In the same cellular system, $\mathrm{LTD}_{4}$ has been demonstrated to induce phosphorylation of apoptosis signal-regulating kinase 1 (Kumasawa et al., 2005), a kinase upstream of c-Jun $\mathrm{NH}_{2}$-terminal kinase (JNK) and p38 MAPK, which in turn regulates transcription factor AP-1, an essential step for regulation of cell proliferation and differentiation. In renal mesangial cells, $\mathrm{LTD}_{4}$-induced proliferation requires ERK1/2 and p38 activation and is dependent on PI3K and PKC (McMahon et al., 2000). LTD $_{4}$ also transactivates the plate- let-derived growth factor receptor $\beta$, which is a process associated with c-Src recruitment and Ras activation, an effect insensitive to PTX and apparently related to CysLT $_{1} R$ activation (McMahon et al., 2002). LTD $_{4}$-induced airway SMC proliferation was also demonstrated to require transactivation of the EGFR through generation of reactive oxygen species (Ravasi et al., 2006). It is noteworthy that very recently $\mathrm{LTD}_{4}$ has been demonstrated to transcriptionally activate VEGF production via CysLT $_{1}$ R, with the involvement of JNK, ERK, the AP-1 complex, and Sp1 (Poulin et al., 2011). Taken together, these findings suggest that cysteinyl LTs may be important in the process of airway remodeling and potentially provide a previously unknown benefit of using LTRAs in the prevention or treatment of chronic asthma, albeit long-term studies aimed to determine its effects on airway remodeling are still lacking. Finally, in human MCs, $\mathrm{LTD}_{4}$ enhanced proliferation in a CysLT ${ }_{1}^{-}$ and ERK-dependent manner, which in turn required transactivation of c-kit (Jiang et al., 2006).

However, in THP-1 cells LTD $_{4}$ has been postulated to activate MAPK through a PKC-Raf-1-dependent pathway (Hoshino et al., 1998), whereas in differentiated U937 cells ERK1/2 activation involves a Ras-GTP-dependent pathway, phospholipase $\mathrm{C}$, and $\mathrm{Ca}^{2+}$-dependent tyrosine kinase(s) (Capra et al., 2004). In addition, $\mathrm{LTD}_{4}$ has been shown to induce proliferation and migration of mouse embryonic stem cells via a mechanism involving STAT-3, PI3K, glycogen synthase kinase-3 $\beta /$ $\beta$-catenin phosphorylation and calcineurin expression (Kim et al., 2010).

In intestinal epithelial cells, $\mathrm{LTD}_{4}$ has been shown to increase cell survival and proliferation (Ohd et al., 2000; Paruchuri and Sjölander, 2003), activating MAPK through a Ras-independent but PKC $\varepsilon$-dependent pathway (Paruchuri et al., 2002), and to increase cell mobility via a PI3K/Rac signaling pathway (Paruchuri et al., 2005). Furthermore, the antiapoptotic effect involves the prevention of caspase 8 activation and Bid cleavage (Wikström et al., 2003a), as well as COX-2 transcription and Bcl-2 up-regulation mediated through a PTX-sensitive $\mathrm{G}$ protein and the ERK-1/2 pathway (Wikström et al., 2003b). Indeed, $\mathrm{LTD}_{4}$ exposure, through PI3K-dependent phosphorylation of glycogen synthase kinase- $3 \beta$ induced a $\beta$-catenin translocation to the nucleus, where there is an elevation of the promoter activity of the TCF/LEF family of transcription factors, and to the mitochondria, where the action is associated with the cell survival protein Bcl-2 (Mezhybovska et al., 2006). CysLT $_{1} R$ involvement in colon cancer cell proliferation was also shown via endogenous production of cysteinyl LTs that, through cytosolic phospholipase $\mathrm{A}_{2}$ activation (Parhamifar et al., 2005), mediates an autocrine survival and proliferation signal in nontumor- and tumorderived epithelial cells (Paruchuri et al., 2006). To the best of our knowledge, there is only one report suggesting that cysteinyl LTs may, on the contrary, inhibit the 
growth of a human cell line (i.e., the mammary cancer MCF-7 cells) (Przylipiak et al., 1998).

Proliferation is not the only effect induced by MAPK activation. For example, in monocytes, $\mathrm{LTD}_{4}$ induced p38 phosphorylation (Woszczek et al., 2008a), a pathway involved in the regulation of immediate-early gene expression. In a human epithelial cell line, CysLT $_{1} R$ activation has been shown to lead to either STAT-1 phosphorylation through PKC and ERK1/2 activation, causing enhanced intercellular cell adhesion molecule-1 expression and eosinophil adhesion (Profita et al., 2008), or to up-regulate mucin gene MUC2 transcription via a signaling pathway involving PKC and NF- $\kappa \mathrm{B}$ (Suzuki et al., 2008). In this respect, $\mathrm{NF}-\kappa \mathrm{B}$, a transcription factor largely involved in inflammation and in regulating the immune response, has been demonstrated to be involved in the $\operatorname{CysLT}_{1} \mathrm{R}$ transduction pathway by different groups. In isolated lung MNCs, for example, $\mathrm{LTD}_{4}$ activated NF- $\kappa \mathrm{B}$ and induced production of RANTES (Kawano et al., 2003), whereas in THP-1 and in human $\mathrm{DCs}_{\mathrm{CysLT}} \mathrm{R}$ engagement induced AP-1- and NF- $\kappa$ Bdependent IL-8 expression (Thompson et al., 2006), as well as increased MCP-1 (Hashimoto et al., 2009). In partial agreement with these data $\mathrm{LTD}_{4}$ has been demonstrated to induce AP-1, but not NF- $\kappa$ B signaling in intestinal epithelial cells (Bengtsson et al., 2008). Therefore, montelukast inhibited NF- $\kappa$ B activation in THP-1 cells in a dose-related manner (Maeba et al., 2005), and TNF- $\alpha$-stimulated IL-8 expression through changes in NF- $\kappa$ B p65-associated histone acetyltransferase activity in differentiated U937 cells (Tahan et al., 2008).

However, three distinct studies have reported that $\mathrm{LTD}_{4}$ did not induce NF- $\mathrm{B}$ nuclear translocation. In contrast, pranlukast inhibited NF- $\kappa \mathrm{B}$ activation (Ichiyama et al., 2003), TNF- $\alpha$ production (Tomari et al., 2003), or MUC2 gene transcription (Ishinaga et al., 2005), effects that were suggested to be independent from $\mathrm{CysLT}_{1} \mathrm{R}$ antagonism. A different study also reported that pranlukast inhibited IL-5 production in various cells irrespective of their CysLT $_{1} \mathrm{R}$ mRNA expression (Fukushima et al., 2005), again suggesting that the compound may have other activities beyond CysLT $_{1} R$ antagonism. Furthermore, montelukast and zafirlukast have been demonstrated to inhibit the effects of nucleotides acting at different $\mathrm{P} 2 \mathrm{Y}$ receptors $\left(\mathrm{P}_{2} \mathrm{Y}_{1,2,4,6}\right)$ (Mamedova et al., 2005), although in a noncompetitive manner. These observations have recently been confirmed by another report demonstrating that montelukast and zafirlukast, acting in a concentrationdependent manner, can inhibit non-CysLT ${ }_{1} R$-mediated proinflammatory reactions in human monocytes while inhibiting UDP-induced $\mathrm{Ca}^{2+}$ mobilization (Woszczek et al., 2010). Taken together, these data demonstrate nonCysLTR-related activities for these classic LTRAs. Thus, the therapeutic effects of these compounds must be considered with some reserve.
Another interesting cellular function that emerged to be modulated by CysLT $_{1} R$ is the up-regulation of $\beta$ integrins. Massoumi and Sjölander (2001) first demonstrated that in intestinal epithelial cells $\mathrm{LTD}_{4}$ induced a Src-dependent rapid tyrosine phosphorylation of vinculin, as well as PKC-dependent up-regulation of active $\beta 1$ integrins on the cell surface and a consequent enhanced adhesion of cells to collagen IV. Furthermore, in Caco-2 cells, $\mathrm{LTD}_{4}$ controlled adhesive properties and migration by up-regulating COX-2 and stimulating $\mathrm{PGE}_{2}$-induced expression of $\alpha 2 \beta 1$ integrins (Massoumi et al., 2003). In another study, $\mathrm{LTD}_{4}$ was demonstrated to rapidly induce focal adhesion kinase-related tyrosine kinase phosphorylation and significantly up-regulated $\alpha 4 \beta 1$ and $\alpha 5 \beta 1$ integrin-dependent adhesion of both primitive and committed hematopoietic stem and progenitor cell (Boehmler et al., 2009).

In some systems, CysLT $_{1} \mathrm{R}$ activation can contribute to the propagation of the inflammatory reaction by the release of various inflammatory mediators and cytokines. Thus, although cytokines may regulate CysLTR expression, cysteinyl LTs may, in turn, induce their release in an amplifying circuit of inflammation. For example, in U937 cells, $\mathrm{LTD}_{4}$ triggered a rapid release of arachidonic acid metabolites into the culture medium, an effect that was suppressed by the CysLTR antagonist 2(S)-hydroxyl-3( $R$ )-carboxyethylthio)-3-[2-(8-phenyloctyl) phenyl] propanoic acid (SKF 104353), by the topoisomerase I inhibitor camptothecin, and by staurosporine (Mattern et al., 1990). Several different groups have reported $\mathrm{CysLT}_{1} \mathrm{R}$-induced release of Th2 cytokines, such as IL-4 from cord blood-derived eosinophils (Bandeira-Melo et al., 2002a,b), IL-5 from MNCs (Nabe et al., 2002; Frieri et al., 2003; Faith et al., 2008), chemokines, such as RANTES, from MNCs (Kawano et al., 2003) or platelets (Hasegawa et al., 2010), IL-8 from THP-1 cells (Thompson et al., 2006), and MIP- $1 \alpha$ and MIP-1 $\beta$ (Ichiyama et al., 2009) or IL-11 (Lee et al., 2007a) from epithelial cells. Of interest, $\mathrm{LTC}_{4}$ and $\mathrm{LTD}_{4}$, but not $\mathrm{LTE}_{4}$, enhanced TNF- $\alpha$-induced MMP-9 production in human MNCs (Ichiyama et al., 2007), an effect that, although inhibited by montelukast, does not seem to be CysLT $_{1}$ R-dependent in eosinophils (Langlois et al., 2006). Finally, a combination of $\mathrm{LTD}_{4}$ and IL-5 or granulocyte macrophage-colony-stimulating factor synergistically induced TGF- $\beta 1$ expression in eosinophils (Kato et al., 2005), whereas $\mathrm{LTC}_{4}$ induced TGF- $\beta 1$ production in airway epithelial cells (Perng et al., 2006) or in a timeand concentration-dependent manner in $\mathrm{CysLT}_{1} \mathrm{R}$ transfected HEK-293 cells (Bossé et al., 2008).

Thus, montelukast and/or pranlukast significantly reduced in OVA-treated mice the increased IL-4 and IL-13 (Henderson et al., 2002; Lee et al., 2007a) in BAL fluid, IL-11 and TGF- $\beta 1$ in BAL and lung tissues (Lee et al., 2007a), IL-4, IL-5, IL-13, and eotaxin in BAL, and TGF- $\beta$ gene expression in GATA-3-overexpressing mice (Kiwamoto et al., 2011), thereby preventing airway in- 
flammation, bronchial hyperresponsiveness, and airway fibrosis. Of interest, LTD $_{4}$ in HEK-293 cells stably transfected with the $\mathrm{CysLT}_{1} \mathrm{R}$ can transcriptionally activate furin production with consequent maturation of furin, a proprotein convertase involved in the maturation/activation of several substrates implicated in the remodeling processes (Thompson et al., 2008b).

Treatment with montelukast decreased the amount of IL-4 but increased the amount of INF- $\gamma$ mRNA expression in the lungs of IL-2-treated OVA-sensitized rats (Nag et al., 2002), decreased tissue inflammation, decreased total cell count and number of eosinophils and lymphocytes in BAL and inhibited the increase in endothelin-1 and INF- $\gamma$ after intratracheal Sephadex provocation in rats (Finsnes et al., 2002). Furthermore, montelukast significantly inhibited LPS-induced IL-6, TNF- $\alpha$, and MCP-1 production in the peripheral blood MNCs of control subjects and patients with asthma (Maeba et al., 2005), whereas pranlukast decreased the levels of many cytokines and chemokines (IL-4, IL-5, IL-1 $\beta$, TNF- $\alpha$, RANTES, and IL-8) in nasal mucosa, leading to improvement in patients with nasal symptoms (Ueda et al., 2003).

2. CysLT $T_{2}$ Receptor. Obtaining detailed information about signal transduction systems involved in CysLT $_{2} R$ activation was hampered by the absence of selective antagonists, at least until a short time ago (Huang et al., 2008; Wunder et al., 2010). Thus, most of the data still rely on either recombinant systems or have been obtained in ECs that predominantly express $\mathrm{CysLT}_{2} \mathrm{R}$. As in the case of the CysLT $_{1} R$, the CysLT $_{2} R$ also seems to be mainly coupled to PI hydrolysis and intracellular $\mathrm{Ca}^{2+}$ mobilization through a PTX-insensitive $\mathrm{G}$ protein $\left(\mathrm{G}_{\mathrm{q} / 11}\right)$ in recombinant systems (Heise et al., 2000). CysLT $_{2} R$ activation in HUVECs causes an increase in $\left[\mathrm{Ca}^{2+}\right]_{\mathrm{i}}$ (Lötzer et al., 2003), confirming early reports in which $\mathrm{LTC}_{4}$ and $\mathrm{LTD}_{4}$ were demonstrated to induce a rapid $\left[\mathrm{Ca}^{2+}\right]_{\mathrm{i}}$ transient that was inhibited by the receptor antagonist pobilukast (Heimbürger and Palmblad, 1996), which, despite being considered a $\mathrm{CysLT}_{1} \mathrm{R}$ antagonist, is apparently a dual $\mathrm{CysLT}_{1} / \mathrm{CysLT}_{2} \mathrm{R}$ antagonist (G. E. Rovati, unpublished observations). Furthermore, in the same cells cysteinyl LTs induce myosin light-chain kinase activation, stress fiber formation, and EC contraction that are totally PTX-insensitive (Carnini et al., 2011). In contrast, in human MCs under conditions in which $\mathrm{CysLT}_{1} \mathrm{R}$ is blocked by MK-571 pretreatment, $\operatorname{CysLT}_{2} \mathrm{R}$ stimulation elicited secretion of IL-8 through p38 activation, an effect completely inhibited by PTX (Mellor et al., 2003).

As mentioned previously, in ECs of human vasculature $\mathrm{CysLT}_{2} \mathrm{R}$ induced relaxation (Allen et al., 1992), an effect that was successively linked to the formation of $\mathrm{NO}$, at least in human pulmonary arteries and veins (Ortiz et al., 1995). In addition to direct action on the vascular tone, cysteinyl LT-induced activation of ECs may also lead to changes in the transcriptional activity.
In HUVECs, $\mathrm{LTD}_{4}$ induces endothelial P-selectin expression (Pedersen et al., 1997), strongly stimulates expression of MIP-2 (Zhao et al., 2004), and together with thrombin up-regulates 37 early inducible genes, among which the most strongly induced are early growth response 1 (EGR1), nuclear receptor subfamily 4 group A transcription factors, E-selectin, CXC ligand 2, IL-8, a disintegrin-like and metalloprotease (reprolysin type) with thrombospondin type 1 motif 1 (ADAMTS1), Down syndrome critical region gene 1 (DSCR1), tissue factor, and COX-2 (Uzonyi et al., 2006). IL-8 induction has been studied in more detail, demonstrating that in HEK-293 cells stably transfected with $\mathrm{CysLT}_{2} \mathrm{R} \mathrm{LTC}_{4}$ transcriptionally activates its production through induction of NF- $\kappa \mathrm{B}$ and AP-1 transcription factors (Thompson et al., 2008a). However, at variance with the results in HUVECs, a recent article suggested that in EA.hy926 cells, a human EC line, $\mathrm{LTD}_{4}$ induced the phosphorylation of ERK1/2, but not that of p38 or JNK and that these effects were blocked by the CysLT $_{1} R$ antagonist montelukast or by the dual antagonist BAY U9773, but not by a novel $\mathrm{CysLT}_{2} \mathrm{R}$ antagonist referred to as Bay cysLT $_{2}$ (Yuan et al., 2009).

\section{Desensitization and Cross-Talk of CysLT Receptors}

Early reports showed that in rat basophilic leukemia cells (Vegesna et al., 1988) and in differentiated U937 cells (Winkler et al., 1988; Pollock and Creba, 1990) treatment with phorbol 12-myristate 13 -acetate reduced $\mathrm{LTD}_{4}$-induced PI metabolism and $\left[\mathrm{Ca}^{2+}\right]_{\mathrm{i}}$ mobilization, whereas inhibitors of PKC selectively enhanced $\mathrm{LTD}_{4}{ }^{-}$ induced $\left[\mathrm{Ca}^{2+}\right]_{\mathrm{i}}$ transients (Winkler et al., 1990). These observations suggested for the first time that PKC might play a role in determining the responsiveness of $\mathrm{CysLT}_{1} \mathrm{R}$. Indeed, native human $\mathrm{CysLT}_{1} \mathrm{R}$ has been demonstrated to be the target for unidirectional extracellular nucleotide-mediated heterologous desensitization (Capra et al., 2005). In fact, ATP/UDP-induced $\mathrm{CysLT}_{1} \mathrm{R}$ desensitization, which was sensitive to PKC inhibition, did not cause receptor internalization and induced a faster recovery of $\mathrm{CysLT}_{1} \mathrm{R}$ functionality with respect to $\mathrm{LTD}_{4}$-induced homologous desensitization. The latter, which was not sensitive to PKC activation by phorbol 12-myristate 13-acetate, induced a fast receptor trafficking and down-regulation, and has been shown to most likely depend on GRK2 activation. Of interest, activation of the CysLT $\mathrm{R}_{1}$ by $\mathrm{LTD}_{4}$ had no effect on $\mathrm{P} 2 \mathrm{Y}$ receptor responses (Capra et al., 2005).

In addition, heterologous desensitization of $\mathrm{CysLT}_{1} \mathrm{R}$ is mediated not only by $\mathrm{G}_{\mathrm{q} / \mathrm{i}}$-coupled receptors but also by $\mathrm{G}_{\mathrm{s}}$-coupled receptors [i.e., $\beta_{2}$-adrenoreceptors $\left(\beta_{2} \mathrm{AR}\right.$ ), histamine $\mathrm{H}_{1 / 2}$ and prostaglandin $\mathrm{EP}_{2 / 4}$ receptors] in differentiated U937 cells and human monocytes, this time through activation of PKA (Capra et al., 2010). Of interest, heterologous desensitization seems to affect mostly the $\mathrm{G}_{\mathrm{i}}$-mediated signaling of the $\mathrm{CysLT}_{1} \mathrm{R}$ (Capra et al., 2005, 2010). 
However, Naik et al. (2005) reported that agonistinduced internalization of recombinant human CysLT $_{1} R$ expressed in HEK-293 cells is GRK/arrestin-independent and significantly PKC-dependent. They also demonstrated that mutation of putative PKC phosphorylation sites in the $\mathrm{C}$-tail of the receptor reduced internalization and increased the signaling, significantly attenuating the effects of PKC inhibition. These findings may reflect the use of recombinant systems that produce varying results, depending on cell type and $G$ protein availability, especially when dealing with GPCRs (Kenakin, 1997). In addition, both PKC $\alpha$ and $\mathrm{PKC} \varepsilon$ have been shown to be activated and involved in the regulation of the $\mathrm{LTD}_{4}$-induced $\mathrm{Ca}^{2+}$ signal in human SMCs (Accomazzo et al., 2001) and intestinal epithelial cells (Thodeti et al., 2001). Therefore, in human airway SMCs, PKC inhibition augmented $\mathrm{LTD}_{4}$-stimulated contraction and increased PI hydrolysis and $\mathrm{Ca}^{2+}$ flux, confirming that PKC can regulate $\mathrm{CysLT}_{1} \mathrm{R}$ responses (Deshpande et al., 2007). Such an integrated network presumably tunes the strength and duration of the inflammatory signals, thereby leading to fine regulation of the physiological responses.

Although a phylogenetic relationship exists between P2YR and CysLTR on the basis of sequence alignment followed by evolutionary analyses (Fredriksson et al., 2003; Kroeze et al., 2003), functional relationships between cysteinyl LT/CysLTR and purine/P2YR systems are still a matter of debate. Mellor et al. (2001, 2002) first suggested that UDP is also able to activate CysLTRs and that UDP and $\mathrm{LTD}_{4}$ mediated ERK activation and cytoprotection, implying a cysteinyl LT-mediated autocrine loop (Jiang et al., 2009). However, UDP was not able to compete for binding sites labeled by $\left[{ }^{3} \mathrm{H}\right] \mathrm{LTD}_{4}$ in COS-7 cells transiently expressing $\mathrm{hCysLT}_{1} \mathrm{R}$. These data, together with the lack of cross-desensitization between CysLT $_{1} \mathrm{R}$ and $\mathrm{P} 2 \mathrm{Y}$ receptors, seem to exclude the involvement of a CysLTR/P2YR heterodimer (Capra et al., 2005). These observations have been recently confirmed by another group using receptor desensitization and small interfering RNA experiments in monocytes and CysLT $_{1} R-$ transfected HEK-293 cells. These investigators reported that $\mathrm{LTD}_{4}$ and UDP were acting on separate receptors (Woszczek et al., 2010). On the other hand, Nonaka et al. (2005) first reported that $\mathrm{LTE}_{4}$ acts as an agonist at the $\mathrm{P}_{2} \mathrm{Y}_{12}$ receptor, data recently confirmed by a different laboratory (Fredman et al., 2010), which seemed to corroborate the demonstration of $\mathrm{LTE}_{4}$-induced pulmonary inflammation mediated through the $\mathrm{P} 2 \mathrm{Y}_{12}$ receptor (Paruchuri et al., 2009). In conclusion, a number of investigations demonstrated a close interplay between these two receptor systems, a topic that may have an impact on the development of therapeutic drugs (see section III.H).

The purinergic receptor, however, is not the only receptor system shown to interact with the CysLTRs. A number of studies have reported that LTRAs used as add-on therapy decrease the need for $\beta_{2}$-agonists (Na- than et al., 1998; Noonan et al., 1998; Jarvis and Markham, 2000; Virchow et al., 2000; Keam et al., 2003; Vaquerizo et al., 2003; Deykin et al., 2007; Borderias et al., 2007) and that a positive association exists between a CysLTR antagonist and a $\beta_{2}$-agonist in controlling asthma symptoms, particularly in patients homozygous for the glycine-16 $\beta_{2} \mathrm{AR}$ polymorphism, which predisposes them to agonist-induced down-regulation and desensitization of the $\beta_{2} \mathrm{AR}$ (Lipworth et al., 2000). The response to $\beta_{2}$-agonist agonists is reduced in asthmatic airways, and this desensitization has been postulated to be due in part to inflammatory mediators such as cysteinyl LTs (Song et al., 1998; Milanese et al., 2005). Indeed, $\mathrm{LTD}_{4}$, through $\mathrm{CysLT}_{1} \mathrm{R}$ activation, induced $\beta_{2} \mathrm{AR}$ desensitization in human airway SMCs in vitro, an effect again mediated by the PKC pathway (Rovati et al., 2006). Another interesting aspect of CysLTR crosstalk is the growth factor receptor transactivation, an aspect already discussed in section III.C.

\section{E. CysLT Receptor Functional Analysis through Altered Gene Expression}

1. Gene Disruption. In 2001, a short isoform and a long isoform of $\mathrm{mCysLT}_{1} \mathrm{R}$ were identified in the mouse (Maekawa et al., 2001; Martin et al., 2001). Comparison of the human and the mouse $\mathrm{CysLT}_{1} \mathrm{R}$ cDNAs demonstrated that human transcript II contains sequences equivalent to the mouse short isoform (Woszczek et al., 2005). Both cDNAs, the human transcript II and the mouse short isoform, are less preferentially expressed (Woszczek et al., 2005). At the same time, the $\mathrm{mCysLT}_{2} \mathrm{R}$ was cloned and two $5^{\prime}$-untranslated region splice variants were identified, with the short form lacking exon 3 as the predominant transcript (Hui et al., 2001). A pharmacological difference was also noted between mouse and human $\mathrm{CysLT}_{2} \mathrm{R}$. Pranlukast, a specific inhibitor for $\mathrm{hCysLT}_{1} \mathrm{R}$, antagonized $\mathrm{mCysLT}_{2} \mathrm{R}$ responses at concentrations of 10 $\mu \mathrm{M}$, as determined by $\left[\mathrm{Ca}^{2+}\right]_{i}$ elevation and receptor-induced promoter activation (Ogasawara et al., 2002).

Soon thereafter, Maekawa et al. (2002) generated $\mathrm{CysLT}_{1} \mathrm{R}$-deficient mice by targeted gene disruption; this resulted in loss of both isoforms of the receptor. Peritoneal macrophages from wild-type mice, which expressed both CysLT $_{1} R$ and CysLT $_{2} R$, responded strongly to $\mathrm{LTD}_{4}$ and slightly to $\mathrm{LTC}_{4}$ in a $\mathrm{Ca}^{2+}$ mobilization assay, whereas those from mutant mice responded to neither ligand. Furthermore, the same authors observed that plasma protein extravasation, but not neutrophil infiltration, was significantly suppressed in $\mathrm{CysLT}_{1} \mathrm{R}$-null mice after zymosan-induced peritonitis or IgE-mediated passive cutaneous anaphylaxis, demonstrating that in acute inflammation the microvasculature responds through the $\mathrm{CysLT}_{1} \mathrm{R}$ to cysteinyl LTs generated in vivo by either monocytes/macrophages or MCs. The finding that $\mathrm{CysLT}_{1} \mathrm{R}$-null mice were not protected against chronic injury induced by bleomycin suggested a proinflammatory role for the $\mathrm{CysLT}_{2} \mathrm{R}$ in the 
chronic response. This finding was corroborated by the observation that bleomycin-induced pulmonary inflammation with fibrosis is significantly attenuated in $\mathrm{LTC}_{4} \mathrm{~S}$-null mice but markedly aggravated in $\mathrm{CysLT}_{1} \mathrm{R}$ null mice (Beller et al., 2004a). The same group of researchers examined this issue by generating CysLT $_{2} R$ deficient mice (Beller et al., 2004b) and found that CysLT $_{2} \mathrm{R}$ is responsible for mediating the contribution of the cysteinyl LTs to bleomycin-induced chronic pulmonary inflammation and fibrosis. In conclusion, these authors unexpectedly found that $\mathrm{CysLT}_{1} \mathrm{R}$ does not mediate the signal for chronic inflammation and fibrosis. They propose that $\mathrm{CysLT}_{1} \mathrm{R}$ is responsible for bronchoconstriction, for microvasculature leakage in acute inflammation, and for a counteracting role in chronic inflammation, whereas the $\operatorname{CysLT}_{2} \mathrm{R}$ is the receptor providing the signal for chronic inflammation. Furthermore, $\mathrm{CysLT}_{2} \mathrm{R}$-targeted gene disruption revealed a significant reduction in vascular permeability (Beller et al., 2004b). These data have been further confirmed in transgenic mouse models (see below).

GPR17-deficient mice sensitized and challenged with dust mite exhibited markedly increased pulmonary inflammatory and serum IgE responses compared with those of wild-type mice. In contrast, a reduced response to this intervention was observed in mice lacking $\mathrm{CysLT}_{1} \mathrm{R}$. These findings reveal a constitutive negative regulation of $\mathrm{CysLT}_{1} \mathrm{R}$ functions by GPR17 in both the antigen presentation and downstream phases of allergic pulmonary inflammation (Maekawa et al., 2010), confirming the in vitro observation of an interplay between GPR17 and CysLT $_{1}$ R (Maekawa et al., 2009) (see section III.H).

2. CysLT Receptor Transgenic Models. A transgenic mouse specifically overexpressing the human CysLT $_{1} R$ in SMCs via the $\alpha$-actin promoter was generated to explore the importance of increased cysteinyl LT signaling in airway function. This transgenic model overcame the problem of mice and isolated murine tracheal rings having a minimal bronchoconstrictor response to direct LT challenge compared with that of guinea pigs or humans (Dahlén et al., 1980; Ashida et al., 1987; Martin et al., 1988). Mice were sensitized by intraperitoneal injections with Aspergillus fumigatus to obtain a model of allergic airway inflammation. The authors observed that in transgenic mice allergen-induced airway hyperresponsiveness was significantly enhanced after $\mathrm{LTD}_{4}$ challenge, thus indicating that overexpression of human $\mathrm{CysLT}_{1} \mathrm{R}$ on SMCs leads to smooth muscle hyperresponsiveness (Yang et al., 2004).

Hui et al. (2004) have generated mice in which the human $\mathrm{CysLT}_{2} \mathrm{R}$ is overexpressed in ECs and reported the effects on endothelial integrity and blood pressure regulation mediated by this receptor subtype in vivo. These researchers observed that, in $\mathrm{CysLT}_{2} \mathrm{R}$ transgenic mice, the permeability response to exogenous $\mathrm{LTC}_{4}$ and to endogenous cysteinyl LTs evoked by passive cutane- ous anaphylaxis was augmented and that this enhanced vascular permeability is controlled via $\mathrm{Ca}^{2+}$ signaling and takes place via a transendothelial vesicle transport mechanism as opposed to a paracellular route (Moos et al., 2008). Furthermore, the rapid, systemic pressor response to intravenous $\mathrm{LTC}_{4}$ was diminished coincidentally with augmented production of NO (Hui et al., 2004). The same group also observed that overexpression of the $\mathrm{hCysLT}_{2} \mathrm{R}$ in mice aggravates myocardial ischemia-reperfusion injury by increasing endothelial permeability and exacerbating inflammatory gene expression, leading to accelerated left ventricular remodeling, induction of peri-infarct zone cellular apoptosis, and impaired cardiac performance (Jiang et al., 2008).

\section{F. Pharmacogenetics of CysLT System}

The characterization of the genes encoding the cysteinyl LT system, including the CysLTR1 and CysLTR2 genes, has advanced the study of CysLTR pharmacology and the GPCR pharmacogenomics of inflammatory disease (Thompson et al., 2005). Furthermore, many of the genes encoding proteins involved in the synthesis and signaling pathways of the cysteinyl LTs are polymorphic and are of interest in genetic studies of asthma (Silverman et al., 2001; Palmer et al., 2002; Israel, 2005).

1. CysLT $T_{1}$ Receptor Pharmacogenetics. The CysLT $_{1} \mathrm{R}$ is a candidate gene in atopic asthma because drugs that act as high-affinity antagonist ligands of the $\mathrm{CysLT}_{1} \mathrm{R}$, such as montelukast, zafirlukast, and pranlukast, have been shown to have a beneficial therapeutic effect in some cases of atopy that result in asthma (for reviews on LTRAs in therapy, see Bousquet et al., 2009; Diamant et al., 2009; Dunn and Goa, 2001; Keam et al., 2003; Wahn and Dass, 2008), allergic rhinitis (AR), or rhinosinusitis (Grayson and Korenblat, 2007) (see section III.I.1). Moreover, approximately 20\% of patients report no change in symptoms during treatment with these agents (Drazen et al., 1999, 2000; Israel, 2005). This finding suggests that in some patients with asthma, the pathway regulating airway cysteinyl LT activity may not be activaed with the clinical symptoms of asthma resulting from the actions of other mediators, which would lead to the clinical phenotype of resistance to LTs modifiers (Asano et al., 2002; Lima et al., 2006; Telleria et al., 2008; Tantisira et al., 2009; Langmack and Martin, 2010).

Although the $\mathrm{CysLT}_{1} \mathrm{R}$ gene may harbor inactivating mutations in some populations, there are many more variants of the CysLT $_{2} \mathrm{R}$ (Thompson et al., 2003). For example, in studies of the people living on the isolated south Atlantic island of Tristan da Cunha, two variants of the CysTL $_{1} R$ have been identified, an I206S variant and an activating G300S variant (Thompson et al., 2007). Although the G300S variant was found to be a mildly activating in response to $\mathrm{LTD}_{4}$ in vitro, it was nonetheless associated with atopy in the Tristan da Cunha population. 
Furthermore, a number of SNPs in the promoter region of the CYSLTR1 gene have been found by different groups. Some SNPs were not associated with either the development of asthma/rhinitis (Choi et al., 2004; Zhang et al., 2006) or the response to treatment (Lee et al., 2007b), whereas others, in particular the $-927 \mathrm{~T}>\mathrm{C}$ polymorphism (Arriba-Mendez et al., 2006; Hao et al., 2006; Sanz et al., 2006; Al-Shemari et al., 2008; Bizzintino et al., 2009), have been postulated to cause differences in transcriptional activity that may be predictors of disease risk (Duroudier et al., 2007; Hong et al., 2009), or treatment response (Kim et al., 2007b) or be associated with AIA (Kim et al., 2006b, 2007a).

As mentioned before, Lynch et al. (1999) mapped the $\mathrm{CysLT}_{1}$ gene to the long arm of the human X chromosome. Therefore, Ohd et al. (2003) found a significantly higher frequency of $\mathrm{CysLT}_{1} \mathrm{R}$ staining in tissues from male patients affected by colorectal adenocarcinoma, suggesting the presence of allele-dependent dysregulation or sex-specific transcriptional subordination. Although there are four SNP variants in the promoter region of the CYSLTR1 gene, two of the haplotypes, termed CAAC and TGGC, constitute more than 95\% of the alleles noted in small population samples. There was no predisposition in patients with asthma or rhinitis to have one haplotype or the other. However, among women with asthma who were homozygous for the CAAC haplotype, there was a significantly higher expression of the CysLT $\mathrm{T}_{1} \mathrm{R}$ transcript I than in women who had the CAAC/TCGC complex genotype; transcript II levels were also significantly lower than those in healthy women homozygous for the CAAC haplotype. These data are concordant with previous findings that TCG and CAA haplotypes in the promoter region are associated with different transcriptional activity (Zhang et al., 2006). Of interest, in a recent report of the British 1958 birth cohort, data suggested that the CYSLTR1 gene promoter polymorphisms might influence the risk of atopy in the female white population with suggestive evidence of heterozygote vigor (Duroudier et al., 2009).

2. CysLT $T_{2}$ Receptor Pharmacogenetics. The CysLT $_{2} \mathrm{R}$ is also a candidate gene for asthma and atopy because it maps to a region of chromosome 13q14 that has been linked to asthma (Kimura et al., 1999) and atopy (Daniels et al., 1996). Specific $\mathrm{CysLT}_{2} \mathrm{R}$ antagonists have only recently been reported (Huang et al., 2008; Wunder et al., 2010), and thus the pharmacological importance of CysLT $_{2} \mathrm{R}$ antagonism in asthma or atopy is yet to be established. However, the development of drugs that target the $\mathrm{CysLT}_{2} \mathrm{R}$ may be useful not only for asthma, given that approximately $20 \%$ of patients fail to respond to $\operatorname{CysLT}_{1} \mathrm{R}$ agents, particularly when the $\mathrm{CysLT}_{2} \mathrm{R}$ is also polymorphic, but also for cardiovascular diseases (Funk, 2005; Moos and Funk, 2008; Riccioni et al., 2008).

The Tristan da Cunha study has identified an atopy associated with the M201V variant, which, unlike the G300S CysLT $\mathrm{T}_{1}$ variant, is partially inactivating in terms of $\left[\mathrm{Ca}^{2+}\right]_{\mathrm{i}}$ signaling (Thompson et al., 2003) and has been demonstrated to have a reduced affinity for $\mathrm{LTC}_{4}$ and particularly for $\mathrm{LTD}_{4}$, an impairment of IL-8 transactivation as well as NF- $\kappa \mathrm{B}$ and JNK phosphorylation (Brochu-Bourque et al., 2011). This association of the CysLT $_{2} \mathrm{R} \mathrm{M201V}$ variant with airway disease has been independently replicated in a large outbred population (Pillai et al., 2004). Transmission disequilibrium testing in 137 Japanese asthmatic families revealed that the $-1220 \mathrm{~A}>\mathrm{C}$ polymorphism of the CYSLTR2 gene is associated with the development of asthma (Fukai et al., 2004), whereas four other sequence variants (either in the $5^{\prime}$ - or $3^{\prime}$-flanking region or downstream of the gene) are associated with AIA (Park et al., 2005) and have been linked to altered $\mathrm{CysLT}_{2} \mathrm{R}$ expression (Shin et al., 2009). It is noteworthy that two other CYSLTR2 gene variants, in association with an $A L O X 5$ polymorphism, have been shown to increase the response to montelukast (Klotsman et al., 2007).

The fact that CysLT $_{1} R$ and CysLT $_{2} R$ are both polymorphic in some individuals suggests that if they are coexpressed on inflammatory cells, they may functionally alter cysteinyl LT signaling. If, like many GPCRs, the CysLT $_{1} R$ and CysLT $_{2} R$ form functional dimers with unique pharmacological properties (Jiang et al., 2007), the $\operatorname{CysLT}_{2} \mathrm{R}$ may also play a role in the $\mathrm{CysLT}_{1} \mathrm{R}$ pharmacology (see section III.G). Paradoxically, in the inbred Tristan da Cunha population the activating CysLT $_{1} R$ G300S variant and the inactivating $\mathrm{CysLT}_{2} \mathrm{R}$ M201V variant seem to interact to confer risk for atopy. In this sample, all double heterozygotes were atopic. General linear modeling indicated that the $\operatorname{CysLT}_{1} \mathrm{R}$ and $\mathrm{CysLT}_{2} \mathrm{R}$ genotypes were independently associated with atopy and asthma phenotypes. The gene-gene interaction, therefore, presents as being consistent with an additive effect that is highly associated with atopy (Thompson et al., 2007).

\section{G. Homodimers or Heterodimers?}

Some experimental evidence indicates the possibility that CysLTR might exist as homodimers and/or heterodimers, on the basis of the observation of dimeric and oligomeric forms of $\mathrm{CysLT}_{1} \mathrm{R}$ in Western blots and the punctate appearance of the immunohistochemical signal in peripheral blood leukocytes (Figueroa et al., 2001) or U937 cells (Capra et al., 2005). In human MCs, which can express both receptor subtypes, Mellor et al. (2003) observed that under conditions in which CysLT $_{1} R$ is blocked, IL-5 generation results only from stimulation with the selective CysLT $_{2} \mathrm{R}$ agonist BAY U9773 and not with cysteinyl LTs, leading them to speculate that this could arise from stimulation of a $\mathrm{CysLT}_{1} / \mathrm{CysLT}_{2}$ heterodimer at a site inaccessible to interference from MK571. Finally, Beller et al., (2004b) postulated, on the basis of the magnitude of the attenuation in IgE-dependent, MC-mediated passive cutaneous anaphylaxis in $\mathrm{CysLT}_{1} \mathrm{R} / \mathrm{CysLT}_{2} \mathrm{R}$-null mice, that the effect observed in 
wild-type littermates was mediated through CysLT $_{1} /$ CysLT $_{2}$ heterodimers. These speculations have been now confirmed by assessment of the formation of a $\mathrm{CysLT}_{1} / \mathrm{CysLT}_{2}$ heterodimer at the nuclear envelope of human MCs. Negative regulation of $\mathrm{CysLT}_{1} \mathrm{R}$-induced mitogenic signaling responses of MCs by $\mathrm{CysLT}_{2} \mathrm{R}$ demonstrates physiologically relevant functions for GPCR heterodimers on primary cells central to inflammation (Jiang et al., 2007). It is noteworthy that recently it has been demonstrated that $\mathrm{CysLT}_{1} \mathrm{R}$ formed heterodimers with its counter-receptor $\mathrm{CysLT}_{2} \mathrm{R}$ under basal conditions and that $\mathrm{LTD}_{4}$ triggers reduced dimerization of CysLTRs in intestinal epithelial cells (Parhamifar et al., 2010).

These observations are of potential interest, considering that GPCR oligomerization influences CysLTR pharmacology and function and offers new horizons for the study of important aspects of cysteinyl LT biology and possibly for the development of new drugs (Dalrymple et al., 2008; Kenakin, 2002; Prinster et al., 2005) (see section III.H).

\section{H. Other Receptors Related to CysLT Receptors}

Over the years, data were reported in the literature to suggest the existence of additional CysLTR subtypes in human tissues (Brink et al., 2003). This proposal was based on classic pharmacodynamic observations on agonist and antagonist potencies and as well as on receptor pharmacological profiles (Rovati et al., 1997; Bäck, 2002; Norel and Brink, 2004; Rovati and Capra, 2007; Austen et al., 2009).

1. GPR17. In an intriguing report Mellor et al. (2001) suggested that in MCs both the $\operatorname{CysLT}_{1} R$ and a yet-unidentified elusive receptor up-regulated by treatment with the proinflammatory cytokine IL-4 were responsive to both cysteinyl LTs and UDP. Pharmacological studies performed with classic $\mathrm{CysLT}_{1} \mathrm{R}$ or the dual $\mathrm{CysLT}_{1} / \mathrm{CysLT}_{2} \mathrm{R}$ antagonists excluded the possibility that this additional receptor was the $\mathrm{CysLT}_{2} \mathrm{R}$ subtype. Subsequently, the hypothesis that the $\mathrm{hCysLT}_{1} \mathrm{R}$ was responding to UDP was also excluded (Capra et al., 2005; Woszczek et al., 2010), whereas different CysLT ${ }_{1} R$ antagonists have been demonstrated to inhibit P2Y receptor signaling (Mamedova et al., 2005; Woszczek et al., 2010).

Screening for orphan GPCRs at an intermediate phylogenetic position between $\mathrm{P} 2 \mathrm{Y}$ and CysLT receptor families revealed that the heterologous expression of GPR17 in $1321 \mathrm{~N} 1$ cells resulted in the generation of an apparently specific and concentration-dependent response to both cysteinyl LTs and extracellular nucleotides (Ciana et al., 2006). In line with previous expression data (Bläsius et al., 1998), both human and rat GPR17 was highly present in brain and in other organs typically undergoing ischemic damage, such as kidney and heart. These data are consistent with the demonstration that both the CysLT $_{1}$ and $\mathrm{P}_{2} \mathrm{Y}_{12 / 13}$ receptor antagonists (montelukast and cangrelor, respectively) or in vivo GPR17 receptor knockdown protected against brain damage in the permanent middle cerebral artery occlusion model of ischemic damage in the rat (Ciana et al., 2006). However, GPR17 has been demonstrated to colocalize and dimerize with CysLT $_{1} R$ on the cell surface of human peripheral blood monocytes and was a negative regulator of the CysLT $_{1} R$ both in vitro (Maekawa et al., 2009) and in vivo (Maekawa et al., 2010), without inducing a specific response, at least when expressed in a recombinant system. A recent article confirmed that GPR17 is indeed activated by uracil nucleotides (Benned-Jensen and Rosenkilde, 2010), although, at least in isolation, it may not respond to cysteinyl LTs (G. E. Rovati, unpublished observations). In addition, in a different native system (differentiated PC12 cells) knock down of GPR17 by small interfering RNA abolished $\mathrm{LTD}_{4}$-induced effect on cell viability (Daniele et al., 2010). In this respect, it might be important to note that $\mathrm{P}_{2} \mathrm{Y}_{6}$ receptors also require an intact cysteinyl LT synthesis and signaling system to induce survival and activation of MCs (Jiang et al., 2009). Thus, CysLT ${ }_{1} \mathrm{R}$ and $\mathrm{P}_{2} \mathrm{Y}_{6} \mathrm{R}$, which are coexpressed on many cell types of innate immunity, seem to reciprocally amplify one another's function through endogenous ligands. In conclusion, whether GPR17 is really a dualistic receptor (a single protomer responding to two different classes of ligands) or may form heterodimers with a P2Y receptor is a matter certainly deserving further investigation.

2. Leukotriene $E_{4}$ Receptor(s). Although $\mathrm{LTE}_{4}$ is only a weak agonist for the defined CysLT $_{1} R$ and CysLT $_{2} R$, this ligand is the most stable and abundant cysteinyl LT in vivo. However, the literature on $\mathrm{LTE}_{4}$-induced effects is conflicting, and there seems to be tissue- and modelrelated differences that remain to be defined. In addition, data from studies in the presence and absence of conversion inhibitors also produced different results (Brink et al., 2003). Early reports indicated greater potency for $\mathrm{LTE}_{4}$ compared with that for the other cysteinyl LTs in constricting guinea pig trachea in vitro (Lewis et al., 1980; Lee et al., 1984) and comparable activity in eliciting a cutaneous wheal and flare response in humans (Soter et al., 1983). Furthermore, patients with AIA responded with enhanced bronchoconstriction to inhalation of $\mathrm{LTE}_{4}$ relative to their responses to $\mathrm{LTC}_{4}$ or histamine (Christie et al., 1993), and inhaled $\mathrm{LTE}_{4}$ caused more tissue and airway eosinophilia than $\mathrm{LTD}_{4}$ (Gauvreau et al., 2001). In contrast, $\mathrm{LTE}_{4}$ failed to activate a CysLTR in human pulmonary vessels (Walch et al., 2002). In 2008, the first report that $\mathrm{LTE}_{4}$ mediated prostaglandin $\mathrm{D}_{2}$ production, which was sensitive to the CysLT $_{1} \mathrm{R}$ antagonist MK-571 but resistant to knock down of this receptor, was published (Paruchuri et al., 2008). Furthermore, this effect, induced through COX-2 induction and ERK, p90RSK, and cAMP-regulated-binding protein phosphorylation, was not mediated by $\mathrm{LTD}_{4}$ but was sensitive to PPAR- $\gamma$ knockdown, suggesting the 
presence of a $\mathrm{LTE}_{4}$-selective receptor in human MCs (Paruchuri et al., 2008). Almost simultaneously it was demonstrated that intradermal injection of $\mathrm{LTE}_{4}$ into the ear of mice deficient in both $\mathrm{CysLT}_{1} \mathrm{R} / \mathrm{CysLT}_{2} \mathrm{R}$ elicited a vascular leak that exceeded the response to intradermal injection of $\mathrm{LTC}_{4}$ or $\mathrm{LTD}_{4}$ and that this response was inhibited by pretreatment of the mice with PTX or a Rho kinase inhibitor (Maekawa et al., 2008). This putative mouse $\mathrm{LTE}_{4}$-specific receptor, however, was not MK-571-sensitive, adding complexity to $\mathrm{LTE}_{4}$ puzzle (Austen et al., 2009). One last piece of the puzzle has been revealed by the same group on the basis of a study in mice lacking both $\mathrm{CysLT}_{1} \mathrm{R} / \mathrm{CysLT}_{2} \mathrm{R}$ : the ADP-reactive $\mathrm{P}_{2} \mathrm{Y}_{12}$ receptor was required for $\mathrm{LTE}_{4}$-mediated pulmonary inflammation. Platelet $\mathrm{P}_{2} \mathrm{Y}_{12}$ receptor expression permitted $\mathrm{LTE}_{4}$-induced activation of ERK in CHO cells and allowed chemokine and prostaglandin $\mathrm{D}_{2}$ production by LAD2 cells, a human MC line. Of interest, competition experiments between radiolabeled ADP and unlabeled $\mathrm{LTE}_{4}$ in this last system seemed to suggest that $\mathrm{P}_{2} \mathrm{Y}_{12}$ must complex with another receptor to recognize $\mathrm{LTE}_{4}$ (Paruchuri et al., 2009). Thus, in the light of the progress on heterodimer pharmacology of GPCRs and their possible physiological significance (Prinster et al., 2005; Gurevich and Gurevich, 2008; Milligan, 2009), the possibility that some of these additional CysLTR subtypes might be the result of the formation of heterodimers with a different pharmacological profile, rather than representing new distinct proteins, should always considered (see section III.H). In all previous cases, these data add complexity to the already established "cross-talk" between the purinergic and the LT receptor systems (Capra et al., 2005; Jiang et al., 2009), suggesting these as possible additional means by which these two receptor systems interact with each other.

\section{New Potential Therapeutic Applications of Leukotriene Receptor Antagonists}

1. Allergic Rhinitis. Many of the cells involved in the pathophysiology of AR produce and release cysteinyl LTs, and a connection between the upper airway disease, rhinitis, and lower airway disease, asthma, has been delineated with the aid of important studies indicating that they essentially represent components of a single inflammatory airway disease (Blaiss, 2005; Thomas, 2006). Molecular and clinical studies also substantiated a role for cysteinyl LTs and their receptors in AR (Peters-Golden and Henderson, 2005; Peters-Golden et al., 2006). Furthermore, beyond the existing connection between $\mathrm{AR}$ and asthma, $\mathrm{AR}$ and chronic rhinosinusitis seem to be related both epidemiologically and symptomatically. In fact, some studies have demonstrated possible involvement of the cysteinyl LT system in chronic rhinosinusitis also (Arango et al., 2002; Higashi et al., 2004), and both $\mathrm{CysLT}_{1}$ and $\mathrm{CysLT}_{2}$ receptors were expressed in various inflammatory cells of patients with active seasonal AR (Figueroa et al., 2003) or rhinosinusitis (Sousa et al., 2002) (see section III.A).

Given the shared pathophysiologies and the frequent coexistence, a common therapeutic approach would seem logical (Greenberger, 2003; Stelmach and Cukier, 2006). Indeed, evidence has begun to accumulate in support of the utility of LTRAs in AR (Wilson et al., 2004; Rodrigo and Yañez, 2006) or rhinosinusitis (Grayson and Korenblat, 2007; Scadding et al., 2008).

Montelukast, which significantly reduced daytime nasal, eye and throat symptoms, nighttime symptoms, and symptoms affecting quality of life (QL) in adult patients with seasonal AR (Grainger and Drake-Lee, 2006; Nayak and Langdon, 2007; Bousquet et al., 2009), has also been demonstrated to be safe in children (Bisgaard et al., 2009). Similar results have also been obtained with zafirlukast (Piatti et al., 2003) and pranlukast (Ueda et al., 2003; Okubo and Baba, 2008).

Other studies indicate that LTRAs can alleviate the signs and symptoms of AR when used either as monotherapy (van Adelsberg et al., 2003; Lee et al., 2004b; Ho and Tan, 2007; Wahn and Dass, 2008; Diamant et al., 2009; Cingi and Ozlugedik, 2010) or in combination with an antihistamine in adults (Meltzer et al., 2000; Nayak et al., 2002; Ciprandi et al., 2004; Kurowski et al., 2004; Ciebiada et al., 2006; Lagos and Marshall, 2007; Li et al., 2009), as well as in children (Phan et al., 2009). Results from meta-analyses indicate that antihistamines and LTRAs are equally effective in improving symptoms of $\mathrm{AR}$ and $\mathrm{QL}$, but that both drugs are less effective than intranasal corticosteroids (Pullerits et al., 2002; Saengpanich et al., 2003; Di Lorenzo et al., 2004b; Wilson et al., 2004; Rodrigo and Yañez, 2006; Van Hoecke et al., 2007). However, in a retrospective observational study, addition of montelukast to current corticosteroid therapy improved long-term asthma control and resulted in substantial reductions in asthma-related resource use by patients with mild or moderate persistent asthma and concomitant seasonal AR (Borderias et al., 2007), confirming previous results on the efficacy of montelukast in patients with AR and asthma (Nayak, 2004; Philip et al., 2004; Price et al., 2006; Virchow and Bachert, 2006; Storms, 2007). Of interest, systemic medication such as montelukast, as expected, provided better relief for symptoms distant from the nasal cavity, whereas the antihistamine reduced rhinorrhea more than either montelukast or budesonide (Sardana et al., 2010). Both second-generation antihistamines and montelukast have been found to be relatively safe and effective in children also (Phan et al., 2009). Montelukast received approval from the U.S. Food and Drug Administration in January 2003 for the treatment of seasonal AR and in 2005 for the treatment of perennial AR.

2. Aspirin/Nonsteroidal Anti-Inflammatory Drug Intolerance. A variable percentage (2-20\%) of adults with asthma exhibit an AIA phenotype (Jawien, 2002), which is the second most frequent untoward allergic reaction to 
drugs (de Weck et al., 2006). AIA consists of the precipitation of asthma and rhinitis attacks in the airways or urticaria and angioedema in the skin in response to aspirin and other nonsteroidal anti-inflammatory drugs (NSAIDs) that inhibit COX-1 (Szczeklik and Stevenson, 2003); selective inhibition of COX-2 does not provoke such responses (Stevenson and Simon, 2001; Gyllfors et al., 2003; Stevenson and Szczeklik, 2006). Within $3 \mathrm{~h}$ of ingestion of aspirin or NSAIDs, individuals with AIA develop bronchoconstriction, often accompanied by rhinorrhea, conjunctival irritation, and scarlet flush (Obase et al., 2005). Compared with normal individuals or subjects with aspirin-tolerant asthma, patients with AIA have increased baseline levels of cysteinyl LTs in urine (Christie et al., 1991; Higashi et al., 2004; Micheletto et al., 2006) exhaled air (Antczak et al., 2002), and in saliva and induced sputum (Gaber et al., 2008), levels that are further enhanced by aspirin challenge (Ortolani et al., 1987; Sladek and Szczeklik, 1993; Szczeklik et al., 1996) and that might be derived, at least in part, from MCs (Sladek and Szczeklik, 1993; O'Sullivan et al., 1996; Mita et al., 2001a). Although a definite explanation for this syndrome is not yet available, a large body of evidence supports the possibility that patients with AIA are particularly dependent on the bronchoprotective and anti-inflammatory properties of prostaglandin $\mathrm{E}_{2}$ (Stevenson and Szczeklik, 2006) and that inhibition of COX-1 in these patients triggers MC activation and release of LTs as well as histamine and tryptase (Picado et al., 1992). In addition to the already discussed high levels of cysteinyl LTs and the localization of CysLT $\mathrm{T}_{1} \mathrm{R}$ in nasal mucosa and leukocytes of patients with AIA (Sousa et al., 2002), the clinical relevance of the cysteinyl LTs in AIA has been supported by studies demonstrating that patients with AIA respond favorably to clinical treatment with LT modifiers (Dahlén et al., 1998, 2002; Israel, 2000; Mastalerz et al., 2002a,b Obase et al., 2002; Lee et al., 2004a; Micheletto et al., 2004; Park et al., 2010).

The possible association between CysLTR1 and CysLTR2 gene polymorphisms and AIA has already been discussed (see section III.F). CysLTR1 gene polymorphism, which is often associated with increased CysLT $_{1} R$ expression, has been found to be associated with AIA in males (Kim et al., 2006b, 2007a). Considering that CysLTR2 gene polymorphisms, which, on the contrary, seem to affect CysLT $_{2} \mathrm{R}$ mRNA transcription and stability (Shin et al., 2009), have also been found to be associated with AIA, particularly when combined with the $\mathrm{LTC}_{4} \mathrm{~S}$ polymorphisms $-444 \mathrm{~A}>\mathrm{C}$ (Park et al., 2005), one might envisage a protective role for CysLT $_{2} \mathrm{R}$ in AIA. These data seem to support the finding that $\mathrm{CysLT}_{2} \mathrm{R}$, interacting with CysLT $_{1} R$, down-modulates cysteinyl LT-dependent responses in MCs (Jiang et al., 2007).

3. Other Atopic Diseases. Beyond asthma and rhinitis, there are other atopic diseases in which cysteinyl LTs are considered to play a role and that normally affect adults and children with relevant human and economic burden, such as atopic dermatitis $(\mathrm{AD})$ and urticaria. Although the role of cysteinyl LTs in the inflammation of $\mathrm{AD}$ is unclear, several reports in the literature, but not all (Veien et al., 2005), detail improvements in patients (adult and children) with moderate to severe AD with the use of LTRAs at the doses generally recommended for asthma treatment (Capra et al., 2006; Leonardi et al., 2007; Broshtilova and Gantcheva, 2010). In support, there is also the evidence that elevated levels of $\mathrm{LTE}_{4}$ have been found in the urine of patients with AD (Adamek-Guzik et al., 2002; Øymar and Aksnes, 2005). Chronic urticaria (CU) may manifest as an idiopathic reaction or as a reaction to a known cause, such as cold, pressure, food additives, or NSAIDs; however, this does not occur with selective COX-2 inhibitors (Zembowicz et al., 2003). Of interest, the prevalence of aspirin sensitivity among patients with $\mathrm{CU}$ is estimated to be between 20 and 30\% (Grattan, 2003), and recently the $\mathrm{LTC}_{4} \mathrm{~S}-444 \mathrm{~A}>\mathrm{C}$ polymorphism has been suggested to be a risk factor for aspirin-induced urticaria (SánchezBorges et al., 2009). Thus, these patients may be more likely to respond to LTRA therapy (Asero, 2000; Pacor et al., 2001). Indeed, although some authors found LTRAs to be ineffective in CU (Reimers et al., 2002; Di Lorenzo et al., 2004a) or even to induce a paradoxical exacerbation (Minciullo et al., 2004; Tedeschi, 2009), montelukast has been demonstrated to be effective in patients for whom treatment with histamine H1-receptor antagonists had proved ineffective (Erbagci, 2002; Bagenstose et al., 2004). These data, therefore, suggest that LTRAs may have benefit for patients with conditions not sufficiently controlled with histamine H1-receptor antagonists (Sanada et al., 2005; Wan, 2009).

4. Cardiovascular Diseases. Biomedical research in the LT field has been markedly polarized to asthma and allergic disorders and has largely overlooked other diseases that are also based on the existence of an inflammatory process with increased vascular permeability and edema, such as CVD (Capra et al., 2007). Although blocking the inflammatory component of any human disease is a long-standing and established concept, the use of LT modifiers (LTRAs and LT synthesis inhibitors) in treating the inflammatory component of CVDs has, surprisingly, been seriously contemplated only in the past few years (Funk, 2005; Peters-Golden and Henderson, 2007; Bäck, 2008b). However, a growing body of evidence suggests a major role for the LTs generated by the 5-LO pathway in the pathogenesis and progression of CVDs (Lötzer et al., 2005; Bäck, 2009a), particularly atherosclerosis, myocardial infarct, stroke, aortic aneurysms, and intimal hyperplasia, as LT signaling is emerging as a crucial component in vascular inflammation (Bäck et al., 2007). Cysteinyl LTs have a unique pharmacological profile, characterized by potent constrictor actions on the microvasculature and thus regulation of blood pressure, enhanced permeability of the 
postcapillary venules, reduced coronary blood flow, reduced myocardial contractility, and cardiac output without affecting the heart rate (for review, see Brink et al., 2003 and Capra et al., 2007).

Both CysLTR subtypes are expressed in diseased human arteries (Allen et al., 1998; Spanbroek et al., 2003). However, the role of $\mathrm{CysLT}_{1} \mathrm{R}$ in CVDs is rather controversial. Findings suggesting a role for cysteinyl LTs in the extension of ischemic damage and in cardiac dysfunction during reperfusion (Toki et al., 1988; Hock et al., 1992) are evenly balanced by results suggesting that these autacoids have no (Hahn et al., 1992) or little (Ito et al., 1989) contribution to the progression of myocardial ischemia-reperfusion injury. On the other hand, the LTRAs investigated in these studies belong to the first generation of molecules and may have not displayed enough potency to compete with the endogenous ligands, which are likely to be present with very high local bioavailability at sites where inflammatory cell accumulation and myocardial injury take place. In fact, the newer potent and commercially available $\mathrm{CysLT}_{1} \mathrm{R}$ antagonist, montelukast, has been found to inhibit atherosclerotic lesion size and intimal hyperplasia (Kaetsu et al., 2007; Jawien et al., 2008) and to reduce vascular reactive oxygen species production, to significantly improve EC function, and to ameliorate atherosclerotic plaque generation in a mouse model in vivo (Mueller et al., 2008). Furthermore, in a rabbit carotid balloon injury model, it significantly reduced the neointima, decreased macrophage content, increased SMC content, and inhibited expression of MCP-1 without having influence on plasma lipids, indicating antiatherogenic effects unrelated to plasma lipid modulation (Ge et al., 2009). A randomized controlled trial of placebo versus either montelukast or theophylline in asthmatic individuals reported significantly lower C-reactive protein in montelukast-treated patients (Allayee et al., 2007).

As regards a role for the $\mathrm{CysLT}_{2} \mathrm{R}$ in CVD, the early, endothelium-targeted overexpression of the human $\mathrm{CysLT}_{2} \mathrm{R}$ in mice aggravates myocardial ischemia-reperfusion injury (Hui et al., 2004), leading to accelerated left ventricular remodeling and impaired cardiac performance (Jiang et al., 2008). Despite expression data suggesting that $\mathrm{CysLT}_{2} \mathrm{R}$ is the main isoform of the receptor for cysteinyl LTs in the normal brain (see section III.A), selective $\mathrm{CysLT}_{1} \mathrm{R}$ antagonists might have a protective effect in focal cerebral ischemia (Yu et al., 2005a,b; Ciana et al., 2006; Qian et al., 2006), possibly decreasing blood-brain barrier permeability (Biber et al., 2009). However, the dual $\mathrm{CysLT}_{1} / \mathrm{CysLT}_{2}$ receptor antagonist BAY U9773 has been proved to be more potent and effective than a selective CysLT $_{1} \mathrm{R}$ antagonist in preventing the brain permeability alteration induced by neutrophil activation (Di Gennaro et al., 2004).

The first report indicated that in vivo inhibition of GPR17 by either montelukast or antisense technology dramatically reduced ischemic damage in a rat model of focal cerebral ischemia (Ciana et al., 2006). Subsequently, it was suggested that GPR17 might orchestrate oligodendrocyte maturation after brain or spinal cord injury at a later stage, indicating a role in fostering brain repair (Lecca et al., 2008; Ceruti et al., 2009, 2011). The latter findings were supported by in vitro experiments showing that GPR17 activation stimulated the maturation of preoligodendrocytes (Lecca et al., 2008; Fumagalli et al., 2011). In contrast, GPR17 transgenic mice exhibited blocked differentiation of neural progenitor cells into oligodendrocytes and also inhibited terminal differentiation of primary oligodendrocyte precursor cells, supporting GPR17 as a negative regulator of oligodendrocyte differentiation and myelination (Chen et al., 2009b). Fumagalli et al. (2011) recently suggested that one possible explanation for the apparent differences between those studies may be that the overexpression of GPR17 occurred with the CNPase promoter in the latter study (Chen et al., 2009b), inducing GPR17 expression at a rather advanced maturation stage. Nevertheless, GPR17-deficient mice exhibit early onset of central nervous system myelination (Chen et al., $2009 \mathrm{~b}$ ), and the exact role of GPR17 signaling in oligodendrocyte maturation and function remains to be determined. Thus, whether GPR17 or indeed cysteinyl LTs are friends or foes in ischemic brain injury still needs to be established (Bäck, 2008a).

5. CysLT in Other Diseases. A number of observations document that 5-LO and its products may be relevant in different diseases either because of an increase in cysteinyl LT production/CysLTR expression or because the use of LTRAs has proved to be efficacious. For example, cysteinyl LTs have been found in the sputum and urine of patients with cystic fibrosis (CF) (Sampson et al., 1990; Spencer et al., 1992), a disease in which the inflammatory process contributes to progressive lung tissue damage, and their concentrations increase during disease exacerbations together with oxidative stress (Reid et al., 2007). Few studies have been conducted to test the hypothesis that LTRAs have the potential to ameliorate $\mathrm{CF}$ lung disease. A pilot study indicated that zafirlukast may benefit adult patients with CF (Conway et al., 2003), whereas montelukast treatment significantly decreased serum (Schmitt-Grohé et al., 2002) and sputum levels of eosinophil cationic protein, IL-8, myeloperoxidase, and increased serum and sputum levels of IL-10 in children (Stelmach et al., 2005). These observations suggest that LTRAs may have measurable antiinflammatory properties and potential to ameliorate $\mathrm{CF}$ lung disease, particularly with long-term use (SchmittGrohé et al., 2007). Chronic inflammation also plays a crucial role also in COPD. However, pulmonary inflammation in COPD is, to some extent, different from that in asthma, because other inflammatory cells (i.e., neutrophils, macrophages, and $\mathrm{CD}^{+} \mathrm{T}$ lymphocytes) are implicated (Gan et al., 2004). In addition, the profile of exhaled eicosanoids may be different from that previ- 
ously reported in asthma: exhaled $\mathrm{PGE}_{2}$ is selectively increased in COPD, whereas $\mathrm{LTE}_{4}$ is increased in asthma (Montuschi et al., 2003), but not in COPD (Gaki et al., 2007). There are only a few studies of the clinical effects of LTRAs in COPD. For example, lung function improvement has been observed with zafirlukast in smokers with COPD (Cazzola et al., 2000; Nannini and Flores, 2003) Furthermore, a retrospective study of patients with moderate to severe COPD, reported that montelukast use improved patient's functional scores (shortness of breath, sputum production, wheezing, and nocturnal symptoms), use of drugs (oral and inhaled corticosteroids, inhaled bronchodilators, and supplemental oxygen), number of visits to the emergency department as well as the number and duration of hospitalizations for acute exacerbations (Rubinstein et al., 2004), and QL (Celik et al., 2005). However, further clinical studies are required to judge the real impact of LTRAs in COPD therapy or at least in a subset of patients with COPD (Usery et al., 2008). Given the significant neutrophilic inflammation in COPD, it is likely that $\mathrm{LTB}_{4}$ has a role in the disease (see also section II.F), and thus BLT antagonists and/or 5-LO/FLAP inhibitors may have greater potential as additional therapy in COPD.

Cysteinyl LTs have been implicated in different liver diseases, such as cholestasis, hepatic inflammation, portal hypertension, and hepatorenal syndrome (Keppler et al., 1988; Farzaneh-Far and Moore, 2003), and their excretion is enhanced in patients with liver cirrhosis and hepatorenal syndrome (Huber et al., 1989). Furthermore, cysteinyl LTs are generated in the liver during the reperfusion period and may contribute to the development of hepatic edema and exert cytotoxicity (Takamatsu et al., 2004). Although inhibition of FLAP abrogated experimental liver injury (Titos et al., 2005) and decreased hepatic inflammation and fibrosis in mice (Horrillo et al., 2007), montelukast was also found to be effective in prevention of liver and intestine injury by reducing apoptosis and oxidative stress in a hepatic ischemia-reperfusion injury model (Daglar et al., 2009) and to improve hepatic fibrosis in cholestatic rats (ElSwefy and Hassanen, 2009).

Finally, the presence of receptors that respond to $\mathrm{LTD}_{4}$ in human detrusor myocytes (Bouchelouche et al., 2001a) and evidence for increased urinary $\mathrm{LTE}_{4}$ in patients with interstitial cystitis and detrusor mastocytosis (Bouchelouche et al., 2001b) may suggest a role for cysteinyl LTs as proinflammatory mediators in this disease. Montelukast treatment resulted in significant improvement in urinary frequency and pain (Bouchelouche et al., 2001c), as well in remission of an eosinophilic cystitis (Sterrett et al., 2006), suggesting a role for LTRAs in managing interstitial cystitis.

LTs are elevated in a number of cancers and some preclinical cancer models have shown efficacy of early LT synthesis inhibitors (Rioux and Castonguay, 1998; Steele et al., 1999). In addition, some reports have advanced the concept that GPCRs are mediators of cell growth by demonstrating their potential to activate MAPKs, particularly ERK1/2 (Marinissen and Gutkind, 2001). Several mitogenic pathways might link GPCRs to the nucleus, some of them requiring the capacity of GPCRs to transactivate a growth factor receptor, such as the EGFR (Daub et al., 1996). Indeed, as already discussed in section III.C.1, CysLT $_{1} R$ activation is able to induce MAPK phosphorylation as well as growth factor receptor transactivation, and, thus, to stimulate proliferation (Wang and Dubois, 2010b). Furthermore, consistent data linking CysLT $_{1} R_{s}$ directly to cancer and block of apoptosis are available for epithelial colon cancer cells (Ohd et al., 2003; Nielsen et al., 2005a; Parhamifar et al., 2005; Magnusson et al., 2007, 2010), whereas little information is available on $\mathrm{CysLT}_{1} \mathrm{R}$ expression in brain tumors, such as astrocytoma, ganglioglioma, and metastatic adenocarcinoma (Zhang et al., 2004), in prostate cancer (Matsuyama et al., 2007) or in breast cancer (Magnusson et al., 2011). Taken together, these findings encourage the study of pharmacological treatment of selected forms of cancer with the LTRAs already marketed for asthma.

\section{Nomenclature for Leukotriene Receptors}

Based on the above-cited studies, the IUPHAR Nomenclature Committee for Leukotriene Receptors concludes that the previously established nomenclature for leukotriene receptors (Brink et al., 2003) remains pertinent. Nevertheless, the present nomenclature may present some limitations, which need to be taken into consideration.

Although 12-HHT is an endogenous ligand for $\mathrm{BLT}_{2} \mathrm{R}$ with higher affinity than $\mathrm{LTB}_{4}$, the classification of this receptor as the low-affinity receptor for $\mathrm{LTB}_{4}$ can be motivated by the important amino acid identity with the high-affinity $\mathrm{BLT}_{1} \mathrm{R}$ and the close genomic localization of the $L T B 4 R$ and LTB4R2 genes. However, considering that $12-\mathrm{HHT}$ is produced in platelets more abundantly than $\mathrm{LTB}_{4}$, the nomenclature of $\mathrm{BLT}_{2} \mathrm{R}$ may be reconsidered in the future.

Second, the interaction between purinergic and leukotriene signaling represents an interesting cross-talk in which leukotrienes may activate purinergic receptors and nucleotides may signal through CysLT receptors. Although there is a phylogenetic relationship between purinergic receptors and leukotriene receptors, the exact interactions between these receptor classes remain to be established. Likewise, classifying the recently deorphanized GPR17 or the $\mathrm{P}_{2} \mathrm{Y}_{12}$ receptor into the leukotriene receptor class seems to be premature at this time. Finally, although functional studies and in vivo experiments indicate that not all leukotriene-induced responses fit the current receptor classification, the molecular structure of the putative leukotriene receptor(s) is presently unknown. 
In conclusion, there is no doubt that leukotriene signaling is complex and may involve several receptor classes. The majority of the known leukotriene-induced effects can be assigned to the currently identified receptor subtypes (Table 1). It is hoped that future studies will extend our current understanding of putative additional leukotriene receptors.

\section{Acknowledgments}

We acknowledge Dr. Charles Brink for his critical reading and helpful advice on the content of this review.

\section{Authorship Contributions}

Wrote or contributed to the writing of the manuscript: Bäck, Dahlén, Drazen, Evans, Serhan, Shimizu, Yokomizo, and Rovati.

\section{References}

Accomazzo MR, Rovati GE, Viganò T, Hernandez A, Bonazzi A, Bolla M, Fumagalli F, Viappiani S, Galbiati E, Ravasi S, et al. (2001) Leukotriene D4-induced activation of smooth-muscle cells from human bronchi is partly $\mathrm{Ca}^{2+}$-independent. Am J Respir Crit Care Med 163:266-272.

Adamek-Guzik T, Guzik TJ, Czerniawska-Mysik G, Korpanty G, Mastalerz L, Radwan J, and Szczeklik A (2002) Urinary leukotriene levels are increased during exacerbation of atopic eczema/dermatitis syndrome. Relation to clinical status. Allergy 57:732-736.

Adams MD, Celniker SE, Holt RA, Evans CA, Gocayne JD, Amanatides PG, Scherer SE, Li PW, Hoskins RA, Galle RF, et al. (2000) The genome sequence of Drosophila melanogaster. Science 287:2185-2195.

Adolfsson JL, Ohd JF, and Sjölander A (1996) Leukotriene D4-induced activation and translocation of the G-protein $\alpha_{\mathrm{i} 3}$-subunit in human epithelial cells. Biochem Biophys Res Commun 226:413-419.

Ahluwalia N, Lin AY, Tager AM, Pruitt IE, Anderson TJ, Kristo F, Shen D, Cruz AR, Aikawa M, Luster AD, et al. (2007) Inhibited aortic aneurysm formation in BLT1deficient mice. J Immunol 179:691-697.

Aiello RJ, Bourassa PA, Lindsey S, Weng W, Freeman A, and Showell HJ (2002) Leukotriene B4 receptor antagonism reduces monocytic foam cells in mice. Arterioscler Thromb Vasc Biol 22:443-449.

Al-Shemari H, Bossé Y, Hudson TJ, Cabaluna M, Duval M, Lemire M, Vallee-Smedja S, Frenkiel S, and Desrosiers M (2008) Influence of leukotriene gene polymorphisms on chronic rhinosinusitis. BMC Med Genet 9:21.

Allayee H, Hartiala J, Lee W, Mehrabian M, Irvin CG, Conti DV, and Lima JJ (2007) The effect of montelukast and low-dose theophylline on cardiovascular disease risk factors in asthmatics. Chest 132:868-874.

Allen S, Dashwood M, Morrison K, and Yacoub M (1998) Differential leukotriene constrictor responses in human atherosclerotic coronary arteries. Circulation 97: 2406-2413.

Allen SP, Chester AH, Piper PJ, Sampson AP, Akl ES, and Yacoub MH (1992) Effects of leukotrienes $\mathrm{C} 4$ and D4 on human isolated saphenous veins. Br J Clin Pharmacol 34:409-414

Alten R, Gromnica-Ihle E, Pohl C, Emmerich J, Steffgen J, Roscher R, Sigmund R Schmolke B, and Steinmann G (2004) Inhibition of leukotriene B4-induced CD11B/CD18 (Mac-1) expression by BIIL 284, a new long acting LTB4 receptor antagonist, in patients with rheumatoid arthritis. Ann Rheum Dis 63:170-176.

Amrani Y, Moore PE, Hoffman R, Shore SA, and Panettieri RA Jr (2001) Interferon- $\gamma$ modulates cysteinyl leukotriene receptor-1 expression and function in human airway myocytes. Am J Respir Crit Care Med 164:2098-2101.

Antczak A, Montuschi P, Kharitonov S, Gorski P, and Barnes PJ (2002) Increased exhaled cysteinyl-leukotrienes and 8-isoprostane in aspirin-induced asthma. Am J Respir Crit Care Med 166:301-306.

Arango P, Borish L, Frierson HF Jr, and Kountakis SE (2002) Cysteinyl leukotrienes in chronic hyperplastic rhinosinusitis. Otolaryngol Head Neck Surg 127:512-515.

Arita M, Ohira T, Sun YP, Elangovan S, Chiang N, and Serhan CN (2007) Resolvin E1 selectively interacts with leukotriene B4 receptor BLT1 and ChemR23 to regulate inflammation. J Immunol 178:3912-3917.

Arm JP (2004) Leukotriene generation and clinical implications. Allergy Asthma Proc 25:37-42.

Arm JP, O'Hickey SP, Spur BW, and Lee TH (1989) Airway responsiveness to histamine and leukotriene E4 in subjects with aspirin-induced asthma. Am Rev Respir Dis 140:148-153.

Arriba-Mendez S, Sanz C, Isidoro-Garcia M, Davild I, Laffond E, Horeno E, Avila C, and Lorente F (2006) 927T $>$ C polymorphism of the cysteinyl-leukotriene type-1 receptor (CYSLTR1) gene in children with asthma and atopic dermatitis. Pediatr Allergy Immunol 17:323-328.

Asano K, Shiomi T, Hasegawa N, Nakamura H, Kudo H, Matsuzaki T, Hakuno H, Fukunaga K, Suzuki Y, Kanazawa M, et al. (2002) Leukotriene C4 synthase gene $\mathrm{A}(-444) \mathrm{C}$ polymorphism and clinical response to a CYS-LT(1) antagonist, pranlukast, in Japanese patients with moderate asthma. Pharmacogenetics 12:565570 .

Asero R (2000) Leukotriene receptor antagonists may prevent NSAID-induced exacerbations in patients with chronic urticaria. Ann Allergy Asthma Immunol 85: $156-157$.

Ashida Y, Nomura M, Kuriki H, and Maki Y (1987) The effect of inhaled leukotriene D4, histamine, or antigen on central and peripheral airways of guinea pigs: analysis of bronchograms with an interactive image analysis system. Eur J Phar macol 141:299-304.

Austen KF (2005) The mast cell and the cysteinyl leukotrienes. Novartis Found Symp 271:166-175.

Austen KF (2007) Additional functions for the cysteinyl leukotrienes recognized through studies of inflammatory processes in null strains. Prostaglandins Other Lipid Mediat 83:182-187.

Austen KF, Maekawa A, Kanaoka Y, and Boyce JA (2009) The leukotriene $\mathrm{E}_{4}$ puzzle: finding the missing pieces and revealing the pathobiologic implications. J Allergy Clin Immunol 124:406-414.

Bäck M (2002) Functional characteristics of cysteinyl-leukotriene receptor subtypes. Life Sci 71:611-622.

Bäck M (2008a) Cysteinyl-leukotrienes in cerebrovascular disease: angels and demons? Arterioscler Thromb Vasc Biol 28:805-806.

Bäck M (2008b) Inflammatory signaling through leukotriene receptors in atherosclerosis. Curr Atheroscler Rep 10:244-251.

Bäck M (2009a) Inhibitors of the 5-lipoxygenase pathway in atherosclerosis. Curr Pharm Des 15:3116-3132.

Bäck M (2009b) Leukotriene signaling in atherosclerosis and ischemia. Cardiovasc Drugs Ther 23:41-48.

Bäck M, Bu DX, Bränström R, Sheikine Y, Yan ZQ, and Hansson GK (2005 Leukotriene B4 signaling through NF- $\kappa$ B-dependent BLT1 receptors on vascula smooth muscle cells in atherosclerosis and intimal hyperplasia. Proc Natl Acad Sci USA 102:17501-17506.

Bäck M, Qiu H, Haeggström JZ, and Sakata K (2004) Leukotriene B4 is an indirectly acting vasoconstrictor in guinea pig aorta via an inducible type of BLT receptor. Am J Physiol Heart Circ Physiol 287:H419-H424.

Bäck M, Sultan A, Ovchinnikova O, and Hansson GK (2007) 5-Lipoxygenaseactivating protein: a potential link between innate and adaptive immunity in atherosclerosis and adipose tissue inflammation. Circ Res 100:946-949.

Bafica A, Scanga CA, Serhan C, Machado F, White S, Sher A, and Aliberti J (2005 Host control of Mycobacterium tuberculosis is regulated by 5-lipoxygenasedependent lipoxin production. J Clin Invest 115:1601-1606.

Bagenstose SE, Levin L, and Bernstein JA (2004) The addition of zafirlukast to cetirizine improves the treatment of chronic urticaria in patients with positive autologous serum skin test results. J Allergy Clin Immunol 113:134-140.

Bandeira-Melo C, Hall JC, Penrose JF, and Weller PF (2002a) Cysteinyl leukotrienes induce IL-4 release from cord blood-derived human eosinophils. J Allergy Clin Immunol 109:975-979.

Bandeira-Melo C and Weller PF (2003) Eosinophils and cysteinyl leukotrienes Prostaglandins Leukot Essent Fatty Acids 69:135-143.

Bandeira-Melo C, Woods LJ, Phoofolo M, and Weller PF (2002b) Intracrine cysteiny leukotriene receptor-mediated signaling of eosinophil vesicular transportmediated interleukin-4 secretion. J Exp Med 196:841-850.

Baneres JL, Martin A, Hullot P, Girard JP, Rossi JC, and Parello J (2003) Structurebased analysis of GPCR function: conformational adaptation of both agonist and receptor upon leukotriene B4 binding to recombinant BLT1. J Mol Biol 329:801814

Bannenberg G and Serhan CN (2010) Specialized pro-resolving lipid mediators in the inflammatory response: an update. Biochim Biophys Acta 1801:1260-1273.

Barone FC, Schmidt DB, Hillegass LM, Price WJ, White RF, Feuerstein GZ, Clark RK, Lee EV, Griswold DE, and Sarau HM (1992) Reperfusion increases neutrophils and leukotriene B4 receptor binding in rat focal ischemia. Stroke 23:13371347; discussion 1347-1348.

Basu S, Jala VR, Mathis S, Rajagopal ST, Del Prete A, Maturu P, Trent JO, and Haribabu B (2007) Critical role for polar residues in coupling leukotriene B4 binding to signal transduction in BLT1. J Biol Chem 282:10005-10017.

Baud L, Goetzl EJ, and Koo CH (1987) Stimulation by leukotriene $\mathrm{D}_{4}$ of increases in the cytosolic concentration of calcium in dimethylsulfoxide-differentiated HL-60 cells. J Clin Invest 80:983-991.

Bautz F, Denzlinger C, Kanz L, and Möhle R (2001) Chemotaxis and transendothelial migration of $\mathrm{CD} 34^{+}$hematopoietic progenitor cells induced by the inflammatory mediator leukotriene D4 are mediated by the 7-transmembrane receptor CysLT1. Blood 97:3433-3440.

Beller TC, Friend DS, Maekawa A, Lam BK, Austen KF, and Kanaoka Y (2004a) Cysteinyl leukotriene 1 receptor controls the severity of chronic pulmonary inflammation and fibrosis. Proc Natl Acad Sci USA 101:3047-3052.

Beller TC, Maekawa A, Friend DS, Austen KF, and Kanaoka Y (2004b) Targeted gene disruption reveals the role of the cysteinyl leukotriene 2 receptor in increased vascular permeability and in bleomycin-induced pulmonary fibrosis in mice. J Biol Chem 279:46129-46134.

Benned-Jensen T and Rosenkilde MM (2010) Distinct expression and ligand-binding profiles of two constitutively active GPR17 splice variants. $\mathrm{Br} J$ Pharmacol 159: 1092-1105.

Bengtsson AM, Massoumi R, and Sjölander A (2008) Leukotriene $\mathrm{D}_{4}$ induces AP-1 but not $\mathrm{NF}_{\kappa} \mathrm{B}$ signaling in intestinal epithelial cells. Prostaglandins Other Lipid Mediat 85:100-106.

Bevan S, Dichgans M, Wiechmann HE, Gschwendtner A, Meitinger T, and Markus HS (2008) Genetic variation in members of the leukotriene biosynthesis pathway confer an increased risk of ischemic stroke: a replication study in two independent populations. Stroke 39:1109-1114.

Biber N, Toklu HZ, Solakoglu S, Gultomruk M, Hakan T, Berkman Z, and Dulger FG (2009) Cysteinyl-leukotriene receptor antagonist montelukast decreases bloodbrain barrier permeability but does not prevent oedema formation in traumatic brain injury. Brain Inj 23:577-584

Birke FW, Meade C.J, Anderskewitz R, Speck GA, and Jennewein HM (2001) In vitro and in vivo pharmacological characterization of BIIL 284, a novel and potent leukotriene $\mathrm{B}_{4}$ receptor antagonist. J Pharmacol Exp Ther 297:458-466.

Bisgaard H, Skoner D, Boza ML, Tozzi CA, Newcomb K, Reiss TF, Knorr B, and Noonan G (2009) Safety and tolerability of montelukast in placebo-controlled pediatric studies and their open-label extensions. Pediatr Pulmonol 44:568-579. 
Bizzintino JA, Khoo SK, Zhang G, Martin AC, Rueter K, Geelhoed GC, Goldblatt J, Laing IA, Le Souëf PN, and Hayden CM (2009) Leukotriene pathway polymorphisms are associated with altered cysteinyl leukotriene production in children with acute asthma. Prostaglandins Leukot Essent Fatty Acids 81:9-15.

Blaiss MS (2005) Rhinitis-asthma connection: epidemiologic and pathophysiologic basis. Allergy Asthma Proc 26:35-40.

Bläsius R, Weber RG, Lichter P, and Ogilvie A (1998) A novel orphan G proteincoupled receptor primarily expressed in the brain is localized on human chromosomal band 2q21. J Neurochem 70:1357-1365.

Boehmler AM, Drost A, Jaggy L, Seitz G, Wiesner T, Denzlinger C, Kanz L, and Möhle R (2009) The CysLT1 ligand leukotriene D4 supports $\alpha 4 \beta 1$ - and $\alpha 5 \beta 1$ mediated adhesion and proliferation of $\mathrm{CD} 34^{+}$hematopoietic progenitor cells. J Immunol 182:6789-6798.

Boivin B, Vaniotis G, Allen BG, and Hébert TE (2008) G protein-coupled receptors in and on the cell nucleus: a new signaling paradigm? J Recept Signal Transduct Res 28:15-28.

Borderias L, Mincewicz G, Paggiaro PL, Guilera M, Sazonov Kocevar V, Taylor SD, and Badia X (2007) Asthma control in patients with asthma and allergic rhinitis receiving add-on montelukast therapy for 12 months: a retrospective observational study. Curr Med Res Opin 23:721-730.

Bortuzzo C, Hanif R, Kashfi K, Staiano-Coico L, Shiff SJ, and Rigas B (1996) The effect of leukotrienes B and selected HETEs on the proliferation of colon cancer cells. Biochim Biophys Acta 1300:240-246.

Bossé Y, Thompson C, McMahon S, Dubois CM, Stankova J, and Rola-Pleszczynski M (2008) Leukotriene D4-induced, epithelial cell-derived transforming growth factor beta1 in human bronchial smooth muscle cell proliferation. Clin Exp Allergy 38:113-121.

Bouchelouche K, Horn T, Nordling J, Larsen S, and Hald T (2001a) The action of cysteinyl-leukotrienes on intracellular calcium mobilization in human detrusor myocytes. BJU Int 87:690-696.

Bouchelouche K, Kristensen B, Nordling J, Horn T, and Bouchelouche P (2001b) Increased urinary leukotriene E4 and eosinophil protein X excretion in patients with interstitial cystitis. $J$ Urol 166:2121-2125.

Bouchelouche K, Nordling J, Hald T, and Bouchelouche P (2001c) The cysteinyl leukotriene D4 receptor antagonist montelukast for the treatment of interstitial cystitis. J Urol 166:1734-1737.

Boulay ME, Duchesneau E, Jacques E, Chakir J, and Boulet LP (2010) CysLT 1 -R expression following allergen provocation in asthma and allergic rhinitis. Prostaglandins Leukot Essent Fatty Acids 83:15-22.

Bousquet J, Demoly P, and Humbert M (2009) Montelukast in guidelines and beyond. Adv Ther 26:575-587.

Braccioni F, Dorman SC, O'byrne PM, Inman MD, Denburg JA, Parameswaran K, Baatjes AJ, Foley R, and Gauvreau GM (2002) The effect of cysteinyl leukotrienes on growth of eosinophil progenitors from peripheral blood and bone marrow of atopic subjects. J Allergy Clin Immunol 110:96-101.

Brach MA, de Vos S, Arnold C, Gruss HJ, Mertelsmann R, and Herrmann F (1992) Leukotriene B4 transcriptionally activates interleukin-6 expression involving NK- $\chi$ B and NF-IL6. Eur J Immunol 22:2705-2711.

Brezinski DA, Nesto RW, and Serhan CN (1992) Angioplasty triggers intracoronary leukotrienes and lipoxin A4. Impact of aspirin therapy. Circulation 86:56-63.

Brink C, Dahlén SE, Drazen J, Evans JF, Hay DW, Nicosia S, Serhan CN, Shimizu $\mathrm{T}$, and Yokomizo T (2003) International Union of Pharmacology. XXXVII: Nomenclature for leukotriene and lipoxin receptors. Pharmacol Rev 55:195-227.

Brochu-Bourque A, Véronneau S, Rola-Pleszczynski M, and Stankova J (2011) Differential signalling defects associated with the M201V polymorphism in the cysteinyl leukotriene type 2 receptor. J Pharmacol Exp Ther 336:431-439.

Broshtilova V and Gantcheva M (2010) Therapeutic Hotline: cysteinyl leukotriene receptor antagonist montelukast in the treatment of atopic dermatitis. Dermatol Ther 23:90-93.

Canetti C, Hu B, Curtis JL, and Peters-Golden M (2003) Syk activation is a leukotriene B4-regulated event involved in macrophage phagocytosis of IgG-coated targets but not apoptotic cells. Blood 102:1877-1883.

Capra V (2004) Molecular and functional aspects of human cysteinyl leukotriene receptors. Pharmacol Res 50:1-11.

Capra V, Accomazzo MR, Gardoni F, Barbieri S, and Rovati GE (2010) A role for inflammatory mediators in heterologous desensitization of CysLT1 receptor in human monocytes. J Lipid Res 51:1075-1084.

Capra V, Accomazzo MR, Ravasi S, Parenti M, Macchia M, Nicosia S, and Rovati GE (2003) Involvement of prenylated proteins in calcium signaling induced by LTD4 in differentiated U937 cells. Prostaglandins Other Lipid Mediat 71:235-251.

Capra V, Ambrosio M, Riccioni G, and Rovati GE (2006) Cysteinyl-leukotriene receptor antagonists: present situation and future opportunities. Curr Med Chem 13:3213-3226.

Capra V, Ravasi S, Accomazzo MR, Citro S, Grimoldi M, Abbracchio MP, and Rovati GE (2005) CysLT1 receptor is a target for extracellular nucleotide-induced heterologous desensitization: a possible feedback mechanism in inflammation. $J$ Cell Sci 118:5625-5636

Capra V, Ravasi S, Accomazzo MR, Parenti M, and Rovati GE (2004) CysLT1 signal transduction in differentiated U937 cells involves the activation of the small GTP-binding protein Ras. Biochem Pharmacol 67:1569-1577.

Capra V, Thompson MD, Sala A, Cole DE, Folco G, and Rovati GE (2007) Cysteinylleukotrienes and their receptors in asthma and other inflammatory diseases: critical update and emerging trends. Med Res Rev 27:469-527.

Carnini C, Accomazzo M, Borroni E, Vitellaro-Zuccarello L, Durand T, Folco G, Rovati G, Capra V, and Sala A (2011) Synthesis of cysteinyl leukotrienes in human endothelial cells: subcellular localization and autocrine signaling through the CysLT2 receptor. FASEB J, in press.

Cazzola M, Boveri B, Carlucci P, Santus P, DiMarco F, Centanni S, and Allegra L (2000) Lung function improvement in smokers suffering from COPD with zafirlu-

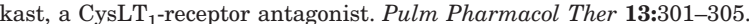

Celik P, Sakar A, Havlucu Y, Yuksel H, Turkdogan P, and Yorgancioglu A (2005)
Short-term effects of montelukast in stable patients with moderate to severe COPD. Respir Med 99:444-450.

Ceruti S, Viganò F, Boda E, Ferrario S, Magni G, Boccazzi M, Rosa P, Buffo A, and Abbracchio MP (2011) Expression of the new P2Y-like receptor GPR17 during oligodendrocyte precursor cell maturation regulates sensitivity to ATP-induced death. Glia 59:363-378.

Ceruti S, Villa G, Genovese T, Mazzon E, Longhi R, Rosa P, Bramanti P, Cuzzocrea S, and Abbracchio MP (2009) The P2Y-like receptor GPR17 as a sensor of damage and a new potential target in spinal cord injury. Brain 132:2206-2218.

Chao SS, Graham SM, Brown CL, Kline JN, and Hussain I (2006) Cysteinyl leukotriene 1 receptor expression in nasal polyps. Ann Otol Rhinol Laryngol 115:394397.

Chen H, Qin J, Wei P, Zhang J, Li Q, Fu L, Li S, Ma C, and Cong B (2009a) Effects of leukotriene $\mathrm{B} 4$ and prostaglandin $\mathrm{E}_{2}$ on the differentiation of murine Foxp3+ T regulatory cells and Th17 cells. Prostaglandins Leukot Essent Fatty Acids 80:195200.

Chen Y, Wu H, Wang S, Koito H, Li J, Ye F, Hoang J, Escobar SS, Gow A, Arnett HA et al. (2009b) The oligodendrocyte-specific G protein-coupled receptor GPR17 is a cell-intrinsic timer of myelination. Nat Neurosci 12:1398-1406.

Chen Z, Gaudreau R, Le Gouill C, Rola-Pleszczynski M, and Stanková J (2004) Agonist-induced internalization of leukotriene $\mathrm{B}_{4}$ receptor 1 requires G-proteincoupled receptor kinase 2 but not arrestins. Mol Pharmacol 66:377-386.

Chiang N, Gronert K, Clish CB, O’Brien JA, Freeman MW, and Serhan CN (1999) Leukotriene B4 receptor transgenic mice reveal novel protective roles for lipoxins and aspirin-triggered lipoxins in reperfusion. J Clin Invest 104:309-316.

Chibana K, Ishii Y, Asakura T, and Fukuda T (2003) Up-regulation of cysteinyl leukotriene 1 receptor by IL-13 enables human lung fibroblasts to respond to leukotriene C4 and produce eotaxin. J Immunol 170:4290-4295.

Cho KJ, Seo JM, Lee MG, and Kim JH (2010a) BLT2 is upregulated in allergenstimulated mast cells and mediates the synthesis of Th2 cytokines. J Immunol 185:6329-6337.

Cho KJ, Seo JM, Shin Y, Yoo MH, Park CS, Lee SH, Chang YS, Cho SH, and Kim JH (2010b) Blockade of airway inflammation and hyperresponsiveness by inhibition of BLT2, a low-affinity leukotriene B4 receptor. Am J Respir Cell Mol Biol 42:294303.

Choi JH, Park HS, Oh HB, Lee JH, Suh YJ, Park CS, and Shin HD (2004) Leukotriene-related gene polymorphisms in ASA-intolerant asthma: an association with a haplotype of 5-lipoxygenase. Hum Genet 114:337-344.

Chou RC, Kim ND, Sadik CD, Seung E, Lan Y, Byrne MH, Haribabu B, Iwakura Y, and Luster $\mathrm{AD}$ (2010) Lipid-cytokine-chemokine cascade drives neutrophil recruitment in a murine model of inflammatory arthritis. Immunity 33:266-278.

Christie PE, Schmitz-Schumann M, Spur BW, and Lee TH (1993) Airway responsiveness to leukotriene C4 (LTC4), leukotriene E4 (LTE4) and histamine in aspirin-sensitive asthmatic subjects. Eur Respir J 6:1468-1473.

Christie PE, Tagari P, Ford-Hutchinson AW, Charlesson S, Chee P, Arm JP, and Lee TH (1991) Urinary leukotriene E4 concentrations increase after aspirin challenge in aspirin-sensitive asthmatic subjects. Am Rev Respir Dis 143:1025-1029.

Ciana P, Fumagalli M, Trincavelli ML, Verderio C, Rosa P, Lecca D, Ferrario S, Parravicini C, Capra V, Gelosa P, et al. (2006) The orphan receptor GPR17 identified as a new dual uracil nucleotides/cysteinyl-leukotrienes receptor. EMBO J 25:4615-4627.

Ciccarelli R, D’Alimonte I, Santavenere C, D'Auro M, Ballerini P, Nargi E, Buccella S, Nicosia S, Folco G, Caciagli F, et al. (2004) Cysteinyl-leukotrienes are released from astrocytes and increase astrocyte proliferation and glial fibrillary acidic protein via cys-LT1 receptors and mitogen-activated protein kinase pathway. Eur $J$ Neurosci 20:1514-1524.

Ciebiada M, Górska-Ciebiada M, DuBuske LM, and Górski P (2006) Montelukast with desloratadine or levocetirizine for the treatment of persistent allergic rhinitis. Ann Allergy Asthma Immunol 97:664-671.

Cingi C and Ozlugedik S (2010) Effects of montelukast on quality of life in patients with persistent allergic rhinitis. Otolaryngol Head Neck Surg 142:654-658.

Cipollone F, Mezzetti A, Fazia ML, Cuccurullo C, Iezzi A, Ucchino S, Spigonardo F Bucci M, Cuccurullo F, Prescott SM, et al. (2005) Association between 5-lipoxygenase expression and plaque instability in humans. Arterioscler Thromb Vasc Biol 25:1665-1670.

Ciprandi G, Tosca MA, Milanese M, Schenone G, and Ricca V (2004) Antihistamines added to an antileukotriene in treating seasonal allergic rhinitis: histamine and leukotriene antagonism. Eur Ann Allergy Clin Immunol 36:67-70, 72.

Cohen P, Noveral JP, Bhala A, Nunn SE, Herrick DJ, and Grunstein MM (1995) Leukotriene D4 facilitates airway smooth muscle cell proliferation via modulation of the IGF axis. Am J Physiol 269:L151-L157.

Conway SP, Etherington C, Peckham DG, and Whitehead A (2003) A pilot study of zafirlukast as an anti-inflammatory agent in the treatment of adults with cystic fibrosis. J Cyst Fibros 2:25-28.

Cookson W (1999) The alliance of genes and environment in asthma and allergy. Nature 402:B5-B11.

Corrigan C, Mallett K, Ying S, Roberts D, Parikh A, Scadding G, and Lee T (2005) Expression of the cysteinyl leukotriene receptors cysLT ${ }_{1}$ and cysLT ${ }_{2}$ in aspirinsensitive and aspirin-tolerant chronic rhinosinusitis. J Allergy Clin Immunol 115:316-322.

Csoma Z, Kharitonov SA, Balint B, Bush A, Wilson NM, and Barnes PJ (2002) Increased leukotrienes in exhaled breath condensate in childhood asthma. Am J Respir Crit Care Med 166:1345-1349.

Currie GP, Srivastava P, Dempsey OJ, and Lee DK (2005) Therapeutic modulation of allergic airways disease with leukotriene receptor antagonists. QJM 98:171182

Daglar G, Karaca T, Yuksek YN, Gozalan U, Akbiyik F, Sokmensuer C, Gurel B, and Kama NA (2009) Effect of montelukast and MK-886 on hepatic ischemiareperfusion injury in rats. J Surg Res 153:31-38.

Dahlén B, Nizankowska E, Szczeklik A, Zetterström O, Bochenek G, Kumlin M, Mastalerz L, Pinis G, Swanson LJ, Boodhoo TI, et al. (1998) Benefits from adding 
the 5-lipoxygenase inhibitor zileuton to conventional therapy in aspirin-intolerant asthmatics. Am J Respir Crit Care Med 157:1187-1194.

Dahlén SE (2006) Treatment of asthma with antileukotrienes: first line or last resort therapy? Eur J Pharmacol 533:40-56.

Dahlén SE, Hedqvist P, Hammarström S, and Samuelsson B (1980) Leukotrienes are potent constrictors of human bronchi. Nature 288:484-486.

Dahlén SE, Malmström K, Nizankowska E, Dahlén B, Kuna P, Kowalski M, Lumry WR, Picado C, Stevenson DD, Bousquet J, et al. (2002) Improvement of aspirinintolerant asthma by montelukast, a leukotriene antagonist: a randomized, double-blind, placebo-controlled trial. Am J Respir Crit Care Med 165:9-14.

Dalrymple MB, Pfleger KD, and Eidne KA (2008) G protein-coupled receptor dimers: functional consequences, disease states and drug targets. Pharmacol Ther 118: 359-371

Daniele S, Lecca D, Trincavelli ML, Ciampi O, Abbracchio MP, and Martini C (2010) Regulation of PC12 cell survival and differentiation by the new P2Y-like receptor GPR17. Cell Signal 22:697-706.

Daniels SE, Bhattacharrya S, James A, Leaves NI, Young A, Hill MR, Faux JA, Ryan GF, le Söuef PN, Lathrop GM, et al. (1996) A genome-wide search for quantitative trait loci underlying asthma. Nature 383:247-250.

Datta YH, Romano M, Jacobson BC, Golan DE, Serhan CN, and Ewenstein BM (1995) Peptido-leukotrienes are potent agonists of von Willebrand factor secretion and P-selectin surface expression in human umbilical vein endothelial cells. Circulation 92:3304-3311.

Daub H, Weiss FU, Wallasch C, and Ullrich A (1996) Role of transactivation of the EGF receptor in signalling by G-protein-coupled receptors. Nature 379:557-560.

de Weck AL, Gamboa PM, Esparza R, and Sanz ML (2006) Hypersensitivity to aspirin and other nonsteroidal anti-inflammatory drugs (NSAIDs). Curr Pharm Des 12:3347-3358.

del Giudice MM, Pezzulo A, Capristo C, Alterio E, Caggiano S, de Benedictis D, and Capristo AF (2009) Leukotriene modifiers in the treatment of asthma in children. Ther Adv Respir Dis 3:245-251.

Del Prete A, Shao WH, Mitola S, Santoro G, Sozzani S, and Haribabu B (2007) Regulation of dendritic cell migration and adaptive immune response by leukotriene B4 receptors: a role for LTB4 in up-regulation of CCR7 expression and function. Blood 109:626-631.

Demitsu T, Katayama H, Saito-Taki T, Yaoita H, and Nakano M (1989) Phagocytosis and bactericidal action of mouse peritoneal macrophages treated with leukotriene B4. Int J Immunopharmacol 11:801-808.

Deshpande DA, Pascual RM, Wang SW, Eckman DM, Riemer EC, Funk CD, and Penn RB (2007) PKC-dependent regulation of the receptor locus dominates functional consequences of cysteinyl leukotriene type 1 receptor activation. FASEB $J$ 21:2335-2342.

Devchand PR, Keller H, Peters JM, Vazquez M, Gonzalez FJ, and Wahli W (1996) The PPAR $\alpha$-leukotriene B4 pathway to inflammation control. Nature 384:39-43.

Deykin A, Wechsler ME, Boushey HA, Chinchilli VM, Kunselman SJ, Craig TJ, DiMango E, Fahy JV, Kraft M, Leone F, et al. (2007) Combination therapy with a long-acting $\beta$-agonist and a leukotriene antagonist in moderate asthma. Am J Respir Crit Care Med 175:228-234.

Di Gennaro A, Carnini C, Buccellati C, Ballerio R, Zarini S, Fumagalli F, Viappiani S, Librizzi L, Hernandez A, Murphy RC, et al. (2004) Cysteinyl-leukotrienes receptor activation in brain inflammatory reactions and cerebral edema formation: a role for transcellular biosynthesis of cysteinyl-leukotrienes. FASEB $J$ 18:842844.

Di Gennaro A, Kenne E, Wan M, Soehnlein O, Lindbom L, and Haeggström JZ (2009) Leukotriene B4-induced changes in vascular permeability are mediated by neutrophil release of heparin-binding protein (HBP/CAP37/azurocidin). FASEB $J$ 23:1750-1757.

Di Lorenzo G, Pacor ML, Mansueto P, Esposito Pellitteri M, Lo Bianco C, Ditta V, Martinelli N, and Rini GB (2004a) Randomized placebo-controlled trial comparing desloratadine and montelukast in monotherapy and desloratadine plus montelukast in combined therapy for chronic idiopathic urticaria. J Allergy Clin Immunol 114:619-625.

Di Lorenzo G, Pacor ML, Pellitteri ME, Morici G, Di Gregoli A, Lo Bianco C, Ditta V, Martinelli N, Candore G, Mansueto P, et al. (2004b) Randomized placebocontrolled trial comparing fluticasone aqueous nasal spray in mono-therapy, fluticasone plus cetirizine, fluticasone plus montelukast and cetirizine plus montelukast for seasonal allergic rhinitis. Clin Exp Allergy 34:259-267.

Diamant Z, Mantzouranis E, and Bjermer L (2009) Montelukast in the treatment of asthma and beyond. Expert Rev Clin Immunol 5:639-658.

Díaz-González F, Alten RH, Bensen WG, Brown JP, Sibley JT, Dougados M, Bombardieri S, Durez P, Ortiz P, de-Miquel G, et al. (2007) Clinical trial of a leucotriene B4 receptor antagonist, BIIL 284, in patients with rheumatoid arthritis. Ann Rheum Dis 66:628-632.

Drazen JM (2003) Leukotrienes in asthma. Adv Exp Med Biol 525:1-5.

Drazen JM, Silverman EK, and Lee TH (2000) Heterogeneity of therapeutic responses in asthma. Br Med Bull 56:1054-1070.

Drazen JM, Yandava CN, Dubé L, Szczerback N, Hippensteel R, Pillari A, Israel E, Schork N, Silverman ES, Katz DA, et al. (1999) Pharmacogenetic association between ALOX5 promoter genotype and the response to anti-asthma treatment. Nat Genet 22:168-170.

Dryden P, Duronio V, Martin L, Hudson AT, and Salari H (1992) Inhibition of human neutrophil responses by $\alpha$-cyano-3,4-dihydroxythiocinnamamide; a proteintyrosine kinase inhibitor. Br J Pharmacol 106:656-664.

Ducharme FM, Lasserson TJ, and Cates CJ (2006) Long-acting beta2-agonists versus anti-leukotrienes as add-on therapy to inhaled corticosteroids for chronic asthma. Cochrane Database Syst Rev 4:CD003137.

Dugas B, Paul-Eugene N, Cairns J, Gordon J, Calenda A, Mencia-Huerta JM, and Braquet P (1990) Leukotriene B4 potentiates the expression and release of $\mathrm{Fc}$ epsilon RII/CD23, and proliferation and differentiation of human B lymphocytes induced by IL-4. J Immunol 145:3406-3411.
Dunn CJ and Goa KL (2001) Zafirlukast: an update of its pharmacology and therapeutic efficacy in asthma. Drugs 61:285-315.

Duroudier NP, Sayers I, Castagna CC, Fenech AG, Halapi E, Swan C, and Hall IP (2007) Functional polymorphism and differential regulation of CYSLTR1 transcription in human airway smooth muscle and monocytes. Cell Biochem Biophys 47:119-130

Duroudier NP, Strachan DP, Blakey JD, and Hall IP (2009) Association of the cysteinyl leukotriene receptor 1 gene with atopy in the British 1958 birth cohort. $J$ Allergy Clin Immunol 124:566-572, 572.e1-572.e3.

Earashi M, Noguchi M, Kinoshita K, and Tanaka M (1995) Effects of eicosanoid synthesis inhibitors on the in vitro growth and prostaglandin $\mathrm{E}$ and leukotriene $\mathrm{B}$ secretion of a human breast cancer cell line. Oncology 52:150-155.

Early SB, Barekzi E, Negri J, Hise K, Borish L, and Steinke JW (2007) Concordant modulation of cysteinyl leukotriene receptor expression by IL- 4 and IFN- $\gamma$ on peripheral immune cells. Am J Respir Cell Mol Biol 36:715-720.

el-Hakim IE, Langdon JD, Zakrzewski JT, and Costello JF (1990) Leukotriene B4 and oral cancer. Br J Oral Maxillofac Surg 28:155-159.

El-Swefy S and Hassanen SI (2009) Improvement of hepatic fibrosis by leukotriene inhibition in cholestatic rats. Ann Hepatol 8:41-49.

Emerson MR and LeVine SM (2004) Experimental allergic encephalomyelitis is exacerbated in mice deficient for 12/15-lipoxygenase or 5-lipoxygenase. Brain Res 1021:140-145.

Erbagci Z (2002) The leukotriene receptor antagonist montelukast in the treatment of chronic idiopathic urticaria: a single-blind, placebo-controlled, crossover clinical study. J Allergy Clin Immunol 110:484-488.

Espinosa K, Bossé Y, Stankova J, and Rola-Pleszczynski M (2003) CysLT1 receptor upregulation by TGF- $\beta$ and IL-13 is associated with bronchial smooth muscle cell proliferation in response to LTD4. J Allergy Clin Immunol 111:1032-1040.

Evans DJ, Barnes PJ, Spaethe SM, van Alstyne EL, Mitchell MI, and O'Connor BJ (1996) Effect of a leukotriene B4 receptor antagonist, LY293111, on allergen induced responses in asthma. Thorax 51:1178-1184.

Evans JF (2003) The cysteinyl leukotriene receptors. Prostaglandins Leukot Essent Fatty Acids 69:117-122.

Evans JF, Ferguson AD, Mosley RT, and Hutchinson JH (2008) What's all the FLAP about?: 5-lipoxygenase-activating protein inhibitors for inflammatory diseases. Trends Pharmacol Sci 29:72-78.

Faccioli LH, Nourshargh S, Moqbel R, Williams FM, Sehmi R, Kay AB, and Williams TJ (1991) The accumulation of ${ }^{111}$ In-eosinophils induced by inflammatory mediators, in vivo. Immunology 73:222-227.

Faith A, Fernandez MH, Caulfield J, Loke TK, Corrigan C, O'Connor B, Lee TH, and Hawrylowicz CM (2008) Role of cysteinyl leukotrienes in human allergen-specific Th2 responses induced by granulocyte macrophage-colony stimulating factor. $\mathrm{Al}$ lergy 63:168-175.

Fang SH, Wei EQ, Zhou Y, Wang ML, Zhang WP, Yu GL, Chu LS, and Chen Z (2006) Increased expression of cysteinyl leukotriene receptor-1 in the brain mediates neuronal damage and astrogliosis after focal cerebral ischemia in rats. Neuroscience 140:969-979.

Fang SH, Zhou Y, Chu LS, Zhang WP, Wang ML, Yu GL, Peng F, and Wei EQ (2007) Spatio-temporal expression of cysteinyl leukotriene receptor-2 mRNA in rat brain after focal cerebral ischemia. Neurosci Lett 412:78-83.

Farzaneh-Far R and Moore K (2003) Cysteinyl-leukotrienes and the liver. Prostaglandins Other Lipid Mediat 72:35-50.

Figueroa DJ, Borish L, Baramki D, Philip G, Austin CP, and Evans JF (2003) Expression of cysteinyl leukotriene synthetic and signalling proteins in inflammatory cells in active seasonal allergic rhinitis. Clin Exp Allergy 33:1380-1388.

Figueroa DJ, Breyer RM, Defoe SK, Kargman S, Daugherty BL, Waldburger K, Liu Q, Clements M, Zeng Z, O'Neill GP, et al. (2001) Expression of the cysteinyl leukotriene 1 receptor in normal human lung and peripheral blood leukocytes. Am J Respir Crit Care Med 163:226-233.

Finsnes F, Lyberg T, Christensen G, and Skjønsberg OH (2002) Leukotriene antagonism reduces the generation of endothelin-1 and interferon-gamma and inhibits eosinophilic airway inflammation. Respir Med 96:901-906.

Flamand L, Tremblay MJ, and Borgeat P (2007) Leukotriene B4 triggers the in vitro and in vivo release of potent antimicrobial agents. J Immunol 178:8036-8045.

Ford-Hutchinson AW, Bray MA, Doig MV, Shipley ME, and Smith MJ (1980) Leukotriene B, a potent chemokinetic and aggregating substance released from polymorphonuclear leukocytes. Nature 286:264-265.

Forman BM, Chen J, and Evans RM (1997) Hypolipidemic drugs, polyunsaturated fatty acids, and eicosanoids are ligands for peroxisome proliferator-activated receptors $\alpha$ and $\delta$. Proc Natl Acad Sci USA 94:4312-4317.

Fredman G, Van Dyke TE, and Serhan CN (2010) Resolvin E1 regulates adenosine diphosphate activation of human platelets. Arterioscler Thromb Vasc Biol 30: 2005-2013.

Fredriksson R, Lagerström MC, Lundin LG, and Schiöth HB (2003) The G-proteincoupled receptors in the human genome form five main families. Phylogenetic analysis, paralogon groups, and fingerprints. Mol Pharmacol 63:1256-1272.

Fregonese L, Silvestri M, Sabatini F, and Rossi GA (2002) Cysteinyl leukotrienes induce human eosinophil locomotion and adhesion molecule expression via a CysLT1 receptor-mediated mechanism. Clin Exp Allergy 32:745-750.

Fretland DJ, Widomski D, Tsai BS, Zemaitis JM, Levin S, Djuric SW, Shone RL, and Gaginella TS (1990) Effect of the leukotriene B4 receptor antagonist SC-41930 on colonic inflammation in rat, guinea pig and rabbit. J Pharmacol Exp Ther 255: $572-576$.

Fretland DJ, Widomski DL, Shone RL, Levin S, and Gaginella TS (1991) Effect of the leukotriene B4 receptor antagonist, SC-41930, on experimental allergic encephalomyelitis (EAE) in the guinea pig. Agents Actions 34:172-174.

Friedrich EB, Tager AM, Liu E, Pettersson A, Owman C, Munn L, Luster AD, and Gerszten RE (2003) Mechanisms of leukotriene B4-triggered monocyte adhesion. Arterioscler Thromb Vasc Biol 23:1761-1767.

Frieri M, Therattil J, Wang SF, Huang CY, and Wang YC (2003) Montelukast inhibits interleukin-5 mRNA expression and cysteinyl leukotriene production in 
ragweed and mite-stimulated peripheral blood mononuclear cells from patients with asthma. Allergy Asthma Proc 24:359-366.

Fujii M, Tanaka H, and Abe S (2005) Interferon- $\gamma$ up-regulates expression of cysteinyl leukotriene type 2 receptors on eosinophils in asthmatic patients. Chest 128: 3148-3155

Fukai H, Ogasawara Y, Migita O, Koga M, Ichikawa K, Shibasaki M, Arinami T, and Noguchi E (2004) Association between a polymorphism in cysteinyl leukotriene receptor 2 on chromosome 13q14 and atopic asthma. Pharmacogenetics 14:683690.

Fukushima C, Matsuse H, Hishikawa Y, Kondo Y, Machida I, Saeki S, Kawano T, Tomari S, Obase Y, Shimoda T, et al. (2005) Pranlukast, a leukotriene receptor antagonist, inhibits interleukin-5 production via a mechanism distinct from leukotriene receptor antagonism. Int Arch Allergy Immunol 136:165-172.

Fumagalli M, Daniele S, Lecca D, Lee PR, Parravicini C, Fields RD, Rosa P Antonucci F, Verderio C, Trincavelli ML, et al. (2011) Phenotypic changes, signaling pathway, and functional correlates of GPR17-expressing neural precursor cells during oligodendrocyte differentiation. J Biol Chem 286:10593-10604.

Funk CD (2005) Leukotriene modifiers as potential therapeutics for cardiovascular disease. Nat Rev Drug Discov 4:664-672.

Gaber F, Daham K, Higashi A, Higashi N, Gülich A, Delin I, James A, Skedinger M Gyllfors P, Nord M, et al. (2008) Increased levels of cysteinyl-leukotrienes in saliva, induced sputum, urine and blood from patients with aspirin-intolerant asthma. Thorax 63:1076-1082.

Gadaleta D, Fantini GA, Silane MF, and Davis JM (1994) Neutrophil leukotriene generation and pulmonary dysfunction after abdominal aortic aneurysm repair. Surgery 116:847-852.

Gagnon L, Filion LG, Dubois C, and Rola-Pleszczynski M (1989) Leukotrienes and macrophage activation: augmented cytotoxic activity and enhanced interleukin 1 , tumor necrosis factor and hydrogen peroxide production. Agents Actions 26:141147.

Gaki E, Papatheodorou G, Ischaki E, Grammenou V, Papa I, and Loukides S (2007) Leukotriene $\mathrm{E}_{4}$ in urine in patients with asthma and COPD—-the effect of smoking habit. Respir Med 101:826-832.

Gan WQ, Man SF, Senthilselvan A, and Sin DD (2004) Association between chronic obstructive pulmonary disease and systemic inflammation: a systematic review and a meta-analysis. Thorax 59:574-580.

Gaudreau R, Beaulieu ME, Chen Z, Le Gouill C, Lavigne P, Stanková J, and Rola-Pleszczynski M (2004) Structural determinants regulating expression of the high affinity leukotriene B4 receptor: involvement of dileucine motifs and $\alpha$-helix VIII. J Biol Chem 279:10338-10345.

Gaudreau R, Le Gouill C, Venne MH, Stankova J, and Rola-Pleszczynski M (2002) Threonine 308 within a putative casein kinase 2 site of the cytoplasmic tail of leukotriene $\mathrm{B}_{4}$ receptor (BLT1) is crucial for ligand-induced, G-protein-coupled receptor-specific kinase 6-mediated desensitization. J Biol Chem 277:3156731576.

Gaudreault E and Gosselin J (2009) Leukotriene B4 potentiates CpG signaling for enhanced cytokine secretion by human leukocytes. J Immunol 183:2650-2658.

Gauvreau GM, Parameswaran KN, Watson RM, and O'Byrne PM (2001) Inhaled leukotriene $\mathrm{E}_{4}$, but not leukotriene $\mathrm{D}_{4}$, increased airway inflammatory cells in subjects with atopic asthma. Am J Respir Crit Care Med 164:1495-1500.

Gauvreau GM, Plitt JR, Baatjes A, and MacGlashan DW (2005) Expression of functional cysteinyl leukotriene receptors by human basophils. J Allergy Clin Immunol 116:80-87.

Ge S, Zhou G, Cheng S, Liu D, Xu J, Xu G, and Liu X (2009) Anti-atherogenic effects of montelukast associated with reduced MCP-1 expression in a rabbit carotid balloon injury model. Atherosclerosis 205:74-79.

Gimbrone MA Jr, Brock AF, and Schafer AI (1984) Leukotriene B4 stimulates polymorphonuclear leukocyte adhesion to cultured vascular endothelial cells. J Clin Invest 74:1552-1555.

Gladue RP, Carroll LA, Milici AJ, Scampoli DN, Stukenbrok HA, Pettipher ER, Salter ED, Contillo L, and Showell HJ (1996) Inhibition of leukotriene B4-receptor interaction suppresses eosinophil infiltration and disease pathology in a murine model of experimental allergic encephalomyelitis. J Exp Med 183:1893-1898.

Goldman DW, Enkel H, Gifford LA, Chenoweth DE, and Rosenbaum JT (1986) Lipopolysaccharide modulates receptors for leukotriene B4, C5a, and formylmethionyl-leucyl-phenylalanine on rabbit polymorphonuclear leukocytes. J Immunol 137:1971-1976.

Goldman DW, Gifford LA, Olson DM, and Goetzl EJ (1985) Transduction by leukotriene B4 receptors of increases in cytosolic calcium in human polymorphonuclear leukocytes. J Immunol 135:525-530.

Goldman DW and Goetzl EJ (1984) Heterogeneity of human polymorphonuclear leukocyte receptors for leukotriene B4. Identification of a subset of high affinity receptors that transduce the chemotactic response. J Exp Med 159:1027-1041.

Goodarzi K, Goodarzi M, Tager AM, Luster AD, and von Andrian UH (2003) Leukotriene B4 and BLT1 control cytotoxic effector T cell recruitment to inflamed tissues. Nat Immunol 4:965-973.

Grainger J and Drake-Lee A (2006) Montelukast in allergic rhinitis: a systematic review and meta-analysis. Clin Otolaryngol 31:360-367.

Grattan CE (2003) Aspirin sensitivity and urticaria. Clin Exp Dermatol 28:123-127. Grayson MH and Korenblat PE (2007) The role of antileukotriene drugs in management of rhinitis and rhinosinusitis. Curr Allergy Asthma Rep 7:209-215.

Greenberger PA (2003) Therapy in the management of the rhinitis/asthma complex. Allergy Asthma Proc 24:403-407.

Griffiths RJ, Pettipher ER, Koch K, Farrell CA, Breslow R, Conklyn MJ, Smith MA, Hackman BC, Wimberly DJ, and Milici AJ (1995) Leukotriene B4 plays a critical role in the progression of collagen-induced arthritis. Proc Natl Acad Sci USA 92:517-521.

Gronert K, Maheshwari N, Khan N, Hassan IR, Dunn M, and Laniado Schwartzman $\mathrm{M}$ (2005) A role for the mouse 12/15-lipoxygenase pathway in promoting epithelial wound healing and host defense. J Biol Chem 280:15267-15278.

Gronert K, Martinsson-Niskanen T, Ravasi S, Chiang N, and Serhan CN (2001)
Selectivity of recombinant human leukotriene $\mathrm{D}_{4}$, leukotriene $\mathrm{B}_{4}$, and lipoxin $\mathrm{A}_{4}$ receptors with aspirin-triggered 15 -epi-LXA $\mathrm{LA}_{4}$ and regulation of vascular and inflammatory responses. Am J Pathol 158:3-9.

Grönroos E, Andersson T, Schippert A, Zheng L, and Sjölander A (1996) Leukotriene $\mathrm{D}_{4}$-induced mobilization of intracellular $\mathrm{Ca}^{2+}$ in epithelial cells is critically dependent on activation of the small GTP-binding protein Rho. Biochem J 316:239-245

Grönroos E, Schippert A, Engström M, and Sjölander A (1995) The regulation of leukotriene D4-induced calcium influx in human epithelial cells involves protein tyrosine phosphorylation. Cell Calcium 17:177-186.

Grönroos E, Thodeti CK, and Sjölander A (1998) Leukotriene D4 induces a rapid increase in cAMP in the human epithelial cell line, Int 407: a potential role for this signal in the regulation of calcium influx through the plasma membrane. Cell Calcium 24:9-16.

Guo Y, Zhang W, Giroux C, Cai Y, Ekambaram P, Dilly AK, Hsu A, Zhou S, Maddipati KR, Liu J, et al. (2011) Identification of the orphan G protein coupled receptor GPR31 as a receptor for $12(S)$ hydroxyeicosatetraenoic acid. J Biol Chem doi:10.1074/jbc.M110.216564

Gurevich VV and Gurevich EV (2008) How and why do GPCRs dimerize? Trends Pharmacol Sci 29:234-240.

Gyllfors P, Bochenek G, Overholt J, Drupka D, Kumlin M, Sheller J, Nizankowska E, Isakson PC, Mejza F, Lefkowith JB, et al. (2003) Biochemical and clinical evidence that aspirin-intolerant asthmatic subjects tolerate the cyclooxygenase 2-selective analgetic drug celecoxib. J Allergy Clin Immunol 111:1116-1121.

Haeggström JZ (2004) Leukotriene A4 hydrolase/aminopeptidase, the gatekeeper of chemotactic leukotriene B4 biosynthesis. J Biol Chem 279:50639-50642.

Hafstrom I, Palmblad J, Malmsten CL, Rådmark O, and Samuelsson B (1981) Leukotriene B4-a stereospecific stimulator for release of lysosomal enzymes from neutrophils. FEBS Lett 130:146-148.

Hahn RA, MacDonald BR, Morgan E, Potts BD, Parli CJ, Rinkema LE, Whitesitt CA, and Marshall WS (1992) Evaluation of LY203647 on cardiovascular leukotriene D4 receptors and myocardial reperfusion injury. J Pharmacol Exp Ther 260:979-989.

Hallstrand TS and Henderson WR Jr (2010) An update on the role of leukotrienes in asthma. Curr Opin Allergy Clin Immunol 10:60-66.

Hanaka H, Shimizu T, and Izumi T (2002) Nuclear-localization-signal-dependent and nuclear-export-signal-dependent mechanisms determine the localization of 5-lipoxygenase. Biochem $J$ 361:505-514.

Hao L, Sayers I, Cakebread JA, Barton SJ, Beghé B, Holgate ST, Sampson AP, and Holloway JW (2006) The cysteinyl-leukotriene type 1 receptor polymorphism 927T/C is associated with atopy severity but not with asthma. Clin Exp Allergy 36:735-741.

Haribabu B, Verghese MW, Steeber DA, Sellars DD, Bock CB, and Snyderman R (2000) Targeted disruption of the leukotriene $B_{4}$ receptor in mice reveals its role in inflammation and platelet-activating factor-induced anaphylaxis. J Exp Med 192: $433-438$.

Haribabu B, Zhelev DV, Pridgen BC, Richardson RM, Ali H, and Snyderman R (1999) Chemoattractant receptors activate distinct pathways for chemotaxis and secretion. Role of G-protein usage. J Biol Chem 274:37087-37092.

Hartiala KT, Langlois L, Goldstein IM, and Rosenbaum JT (1985) Endotoxininduced selective dysfunction of rabbit polymorphonuclear leukocytes in response to endogenous chemotactic factors. Infect Immun 50:527-533.

Hasegawa S, Ichiyama T, Hashimoto K, Suzuki Y, Hirano R, Fukano R, and Furukawa $\mathrm{S}$ (2010) Functional expression of cysteinyl leukotriene receptors on human platelets. Platelets 21:253-259.

Hashidate T, Murakami N, Nakagawa M, Ichikawa M, Kurokawa M, Shimizu T, and Nakamura M (2010) AML1 enhances the expression of leukotriene B4 type-1 receptor in leukocytes. FASEB $J$ 24:3500-3510.

Hashimoto A, Endo H, Hayashi I, Murakami Y, Kitasato H, Kono S, Matsui T, Tanaka S, Nishimura A, Urabe K, et al. (2003) Differential expression of leukotriene B4 receptor subtypes (BLT1 and BLT2) in human synovial tissues and synovial fluid leukocytes of patients with rheumatoid arthritis. J Rheumatol 30:1712-1718.

Hashimoto K, Ichiyama T, Hasegawa M, Hasegawa S, Matsubara T, and Furukawa S (2009) Cysteinyl leukotrienes induce monocyte chemoattractant protein-1 in human monocyte/macrophages via mitogen-activated protein kinase and nuclear factor- $\kappa$ B pathways. Int Arch Allergy Immunol 149:275-282.

Hébert MJ, Takano T, Holthöfer H, and Brady HR (1996) Sequential morphologic events during apoptosis of human neutrophils. Modulation by lipoxygenasederived eicosanoids. J Immunol 157:3105-3115.

Heimbürger M and Palmblad JE (1996) Effects of leukotriene C4 and D4, histamine and bradykinin on cytosolic calcium concentrations and adhesiveness of endothelial cells and neutrophils. Clin Exp Immunol 103:454-460.

Heise CE, O'Dowd BF, Figueroa DJ, Sawyer N, Nguyen T, Im DS, Stocco R, Bellefeuille JN, Abramovitz M, Cheng R, et al. (2000) Characterization of the human cysteinyl leukotriene 2 receptor. J Biol Chem 275:30531-30536.

Heller EA, Liu E, Tager AM, Sinha S, Roberts JD, Koehn SL, Libby P, Aikawa ER, Chen JQ, Huang P, et al. (2005) Inhibition of atherogenesis in BLT1-deficient mice reveals a role for LTB4 and BLT1 in smooth muscle cell recruitment. Circulation 112:578-586.

Henderson WR Jr, Tang LO, Chu SJ, Tsao SM, Chiang GK, Jones F, Jonas M, Pae C, Wang H, and Chi EY (2002) A role for cysteinyl leukotrienes in airway remodeling in a mouse asthma model. Am J Respir Crit Care Med 165:108-116.

Hennig R, Ding XZ, Tong WG, Schneider MB, Standop J, Friess H, Büchler MW, Pour PM, and Adrian TE (2002) 5-Lipoxygenase and leukotriene $\mathrm{B}_{4}$ receptor are expressed in human pancreatic cancers but not in pancreatic ducts in normal tissue. Am J Pathol 161:421-428.

Hennig R, Ding XZ, Tong WG, Witt RC, Jovanovic BD, and Adrian TE (2004) Effect of LY293111 in combination with gemcitabine in colonic cancer. Cancer Lett 210:41-46.

Hennig R, Ventura J, Segersvard R, Ward E, Ding XZ, Rao SM, Jovanovic BD, Iwamura T, Talamonti MS, Bell RH Jr, et al. (2005) LY293111 improves efficacy 
of gemcitabine therapy on pancreatic cancer in a fluorescent orthotopic model in athymic mice. Neoplasia 7:417-425.

Higashi N, Taniguchi M, Mita H, Kawagishi Y, Ishii T, Higashi A, Osame M, and Akiyama K (2004) Clinical features of asthmatic patients with increased urinary leukotriene E4 excretion (hyperleukotrienuria): involvement of chronic hyperplastic rhinosinusitis with nasal polyposis. J Allergy Clin Immunol 113:277-283.

Hikiji H, Ishii S, Yokomizo T, Takato T, and Shimizu T (2009) A distinctive role of the leukotriene B4 receptor BLT1 in osteoclastic activity during bone loss. Proc Natl Acad Sci USA 106:21294-21299.

Hlawaty H, Jacob MP, Louedec L, Letourneur D, Brink C, Michel JB, Feldman L, and Bäck M (2009) Leukotriene receptor antagonism and the prevention of extracellular matrix degradation during atherosclerosis and in-stent stenosis. Arterioscler Thromb Vasc Biol 29:518-524.

Ho CY and Tan CT (2007) Comparison of antileukotrienes and antihistamines in the treatment of allergic rhinitis. Am J Rhinol 21:439-443.

Hock CE, Beck LD, and Papa LA (1992) Peptide leukotriene receptor antagonism in myocardial ischaemia and reperfusion. Cardiovasc Res 26:1206-1211.

Holgate ST, Peters-Golden M, Panettieri RA, and Henderson WR Jr (2003) Roles of cysteinyl leukotrienes in airway inflammation, smooth muscle function, and remodeling. J Allergy Clin Immunol 111:S18-S36.

Hong X, Zhou H, Tsai HJ, Wang X, Liu X, Wang B, Xu X, and Xu X (2009) Cysteinyl leukotriene receptor 1 gene variation and risk of asthma. Eur Respir J 33:42-48.

Hoover RL, Karnovsky MJ, Austen KF, Corey EJ, and Lewis RA (1984) Leukotriene B4 action on endothelium mediates augmented neutrophil/endothelial adhesion. Proc Natl Acad Sci USA 81:2191-2193.

Horrillo R, Planagumà A, González-Périz A, Ferré N, Titos E, Miquel R, López-Parra M, Masferrer JL, Arroyo V, and Clària J (2007) Comparative protection against liver inflammation and fibrosis by a selective cyclooxygenase-2 inhibitor and a nonredox-type 5-lipoxygenase inhibitor. J Pharmacol Exp Ther 323:778-786.

Hoshino M, Izumi T, and Shimizu T (1998) Leukotriene D4 activates mitogenactivated protein kinase through a protein kinase $\mathrm{C} \alpha$-Raf-1-dependent pathway in human monocytic leukemia THP-1 cells. J Biol Chem 273:4878-4882.

Houard X, Ollivier V, Louedec L, Michel JB, and Bäck M (2009) Differential inflammatory activity across human abdominal aortic aneurysms reveals neutrophilderived leukotriene B4 as a major chemotactic factor released from the intraluminal thrombus. FASEB J 23:1376-1383.

Huang L, Zhao A, Wong F, Ayala JM, Struthers M, Ujjainwalla F, Wright SD, Springer MS, Evans J, and Cui J (2004) Leukotriene B4 strongly increases monocyte chemoattractant protein-1 in human monocytes. Arterioscler Thromb Vasc Biol 24:1783-1788.

Huang WW, Garcia-Zepeda EA, Sauty A, Oettgen HC, Rothenberg ME, and Luster $\mathrm{AD}$ (1998) Molecular and biological characterization of the murine leukotriene B4 receptor expressed on eosinophils. J Exp Med 188:1063-1074.

Huang XJ, Zhang WP, Li CT, Shi WZ, Fang SH, Lu YB, Chen Z, and Wei EQ (2008) Activation of CysLT receptors induces astrocyte proliferation and death after oxygen-glucose deprivation. Glia 56:27-37.

Huber M, Kästner S, Schölmerich J, Gerok W, and Keppler D (1989) Analysis of cysteinyl leukotrienes in human urine: enhanced excretion in patients with liver cirrhosis and hepatorenal syndrome. Eur J Clin Invest 19:53-60.

Hui Y, Cheng Y, Smalera I, Jian W, Goldhahn L, Fitzgerald GA, and Funk CD (2004) Directed vascular expression of human cysteinyl leukotriene 2 receptor modulates endothelial permeability and systemic blood pressure. Circulation 110:3360-3366

Hui Y, Yang G, Galczenski H, Figueroa DJ, Austin CP, Copeland NG, Gilbert DJ, Jenkins NA, and Funk CD (2001) The murine cysteinyl leukotriene 2 (CysLT2) receptor. cDNA and genomic cloning, alternative splicing, and in vitro characterzation. J Biol Chem 276:47489-47495.

Hung CH, Li CY, Hua YM, Chen CJ, Yang KD, and Jong YJ (2006) Effects of leukotriene receptor antagonists on monocyte chemotaxis, p38 and cytoplasmic calcium. Pediatr Allergy Immunol 17:250-258.

Hwang SW, Cho H, Kwak J, Lee SY, Kang CJ, Jung J, Cho S, Min KH, Suh YG, Kim D, et al. (2000) Direct activation of capsaicin receptors by products of lipoxygenases: endogenous capsaicin-like substances. Proc Natl Acad Sci USA 97:61556160.

Ichiyama T, Hasegawa M, Hashimoto K, Matsushige T, Hirano R, and Furukawa S (2009) Cysteinyl leukotrienes induce macrophage inflammatory protein-1 in human monocytes/macrophages. Int Arch Allergy Immunol 148:147-153.

Ichiyama T, Hasegawa M, Ueno Y, Makata H, Matsubara T, and Furukawa S (2005) Cysteinyl leukotrienes induce monocyte chemoattractant protein 1 in human monocytes/macrophages. Clin Exp Allergy 35:1214-1219.

Ichiyama T, Hasegawa S, Umeda M, Terai K, Matsubara T, and Furukawa S (2003) Pranlukast inhibits NF- $\kappa \mathrm{B}$ activation in human monocytes/macrophages and T cells. Clin Exp Allergy 33:802-807.

Ichiyama T, Kajimoto M, Hasegawa M, Hashimoto K, Matsubara T, and Furukawa S (2007) Cysteinyl leukotrienes enhance tumour necrosis factor- $\alpha$-induced matrix metalloproteinase-9 in human monocytes/macrophages. Clin Exp Allergy 37:608614.

Ihaku D, Cameron L, Suzuki M, Molet S, Martin J, and Hamid Q (1999) Montelukast, a leukotriene receptor antagonist, inhibits the late airway response to antigen, airway eosinophilia, and IL-5-expressing cells in Brown Norway rats. $J$ Allergy Clin Immunol 104:1147-1154.

Ihara A, Wada K, Yoneda M, Fujisawa N, Takahashi H, and Nakajima A (2007) Blockade of leukotriene B4 signaling pathway induces apoptosis and suppresses cell proliferation in colon cancer. J Pharmacol Sci 103:24-32.

Iizuka Y, Okuno T, Saeki K, Uozaki H, Okada S, Misaka T, Sato T, Toh H, Fukayama M, Takeda N, et al. (2010) Protective role of the leukotriene B4 receptor BLT2 in murine inflammatory colitis. FASEB $J$ 24:4678-4690.

Iizuka Y, Yokomizo T, Terawaki K, Komine M, Tamaki K, and Shimizu T (2005) Characterization of a mouse second leukotriene B4 receptor, mBLT2: BLT2 dependent ERK activation and cell migration of primary mouse keratinocytes. $J$ Biol Chem 280:24816-24823.

Ishinaga H, Takeuchi K, Kishioka C, Suzuki S, Basbaum C, and Majima Y (2005)
Pranlukast inhibits NF- $\kappa$ B activation and MUC2 gene expression in cultured human epithelial cells. Pharmacology 73:89-96.

Islam SA, Thomas SY, Hess C, Medoff BD, Means TK, Brander C, Lilly CM, Tager $\mathrm{AM}$, and Luster $\mathrm{AD}$ (2006) The leukotriene B4 lipid chemoattractant receptor BLT1 defines antigen-primed T cells in humans. Blood 107:444-453.

Israel E (2000) The protective effects of leukotriene modifiers in aspirin-induced asthma. Postgrad Med 108:40-44.

Israel $\mathrm{E}(2005)$ Genetics and the variability of treatment response in asthma. $J$ Allergy Clin Immunol 115:S532-S538.

Ito S, Ito Y, Katagiri H, Suzuki T, Hoka S, Yokomizo T, Shimizu T, and Majima M (2008) Leukotriene B4/leukotriene B4 receptor pathway is involved in hepatic microcirculatory dysfunction elicited by endotoxin. Shock 30:87-91.

Ito T, Toki Y, Hieda N, Okumura K, Hashimoto H, Ogawa K, and Satake T (1989) Protective effects of a thromboxane synthetase inhibitor, a thromboxane antagonist, a lipoxygenase inhibitor and a leukotriene C4, D4 antagonist on myocardia injury caused by acute myocardial infarction in the canine heart. Jpn Circ $J$ 53:1115-1121.

Jala VR, Shao WH, and Haribabu B (2005) Phosphorylation-independent $\beta$-arrestin translocation and internalization of leukotriene B4 receptors. J Biol Chem 280: $4880-4887$.

James AJ, Penrose JF, Cazaly AM, Holgate ST, and Sampson AP (2006) Human bronchial fibroblasts express the 5-lipoxygenase pathway. Respir Res 7:102.

Jarvis B and Markham A (2000) Montelukast: a review of its therapeutic potential in persistent asthma. Drugs 59:891-928.

Jawien J (2002) A new insight into aspirin-induced asthma. Eur J Clin Invest 32:134-138.

Jawien J, Gajda M, Wołkow P, Zurańska J, Olszanecki R, and Korbut R (2008) The effect of montelukast on atherogenesis in apoE/LDLR-double knockout mice $J$ Physiol Pharmacol 59:633-639.

Jiang W, Hall SR, Moos MP, Cao RY, Ishii S, Ogunyankin KO, Melo LG, and Funk CD (2008) Endothelial cysteinyl leukotriene 2 receptor expression mediates myocardial ischemia-reperfusion injury. Am $J$ Pathol 172:592-602.

Jiang Y, Borrelli L, Bacskai BJ, Kanaoka Y, and Boyce JA (2009) P2Y6 receptor require an intact cysteinyl leukotriene synthetic and signaling system to induce survival and activation of mast cells. J Immunol 182:1129-1137.

Jiang Y, Borrelli LA, Kanaoka Y, Bacskai BJ, and Boyce JA (2007) CysLT2 receptor interact with CysLT1 receptors and down-modulate cysteinyl leukotriene dependent mitogenic responses of mast cells. Blood 110:3263-3270

Jiang Y, Kanaoka Y, Feng C, Nocka K, Rao S, and Boyce JA (2006) Cutting edge: Interleukin 4-dependent mast cell proliferation requires autocrine/intracrine cysteinyl leukotriene-induced signaling. J Immunol 177:2755-2759.

Joost P and Methner A (2002) Phylogenetic analysis of 277 human G-protein-coupled receptors as a tool for the prediction of orphan receptor ligands. Genome Biol 3:RESEARCH0063.

Jupp J, Hillier K, Elliott DH, Fine DR, Bateman AC, Johnson PA, Cazaly AM, Penrose JF, and Sampson AP (2007) Colonic expression of leukotriene-pathway enzymes in inflammatory bowel diseases. Inflamm Bowel Dis 13:537-546.

Kaetsu Y, Yamamoto Y, Sugihara S, Matsuura T, Igawa G, Matsubara K, Igawa O, Shigemasa C, and Hisatome I (2007) Role of cysteinyl leukotrienes in the proliferation and the migration of murine vascular smooth muscle cells in vivo and in vitro. Cardiovasc Res 76:160-166.

Kamohara M, Takasaki J, Matsumoto M, Matsumoto Si, Saito T, Soga T, Matsushime H, and Furuichi K (2001) Functional characterization of cysteinyl leukotriene $\mathrm{CysLT}_{2}$ receptor on human coronary artery smooth muscle cells. Biochem Biophys Res Commun 287:1088-1092.

Kamohara M, Takasaki J, Matsumoto M, Saito T, Ohishi T, Ishii H, and Furuichi K (2000) Molecular cloning and characterization of another leukotriene B4 receptor. J Biol Chem 275:27000-27004

Kaneko I, Suzuki K, Matsuo K, Kumagai H, Owada Y, Noguchi N, Hishinuma T, and Ono M (2009) Cysteinyl leukotrienes enhance the degranulation of bone marrowderived mast cells through the autocrine mechanism. Tohoku J Exp Med 217:185191.

Kato K, Yokomizo T, Izumi T, and Shimizu T (2000) Cell-specific transcriptional regulation of human leukotriene $\mathrm{B}_{4}$ receptor gene. J Exp Med 192:413-420.

Kato Y, Fujisawa T, Nishimori H, Katsumata H, Atsuta J, Iguchi K, and Kamiya H (2005) Leukotriene D4 induces production of transforming growth factor-beta1 by eosinophils. Int Arch Allergy Immunol 137 (Suppl 1):17-20.

Kawano T, Matsuse H, Kondo Y, Machida I, Saeki S, Tomari S, Mitsuta K, Obase Y, Fukushima C, Shimoda T, et al. (2003) Cysteinyl leukotrienes induce nuclear factor $\kappa \mathrm{B}$ activation and RANTES production in a murine model of asthma. $J$ Allergy Clin Immunol 112:369-374.

Keam SJ, Lyseng-Williamson KA, and Goa KL (2003) Pranlukast: a review of its use in the management of asthma. Drugs 63:991-1019.

Kelefiotis D, Bresnahan BA, Stratidakis I, and Lianos EA (1995) Eicosanoid-induced growth and signaling events in rat glomerular mesangial cells. Prostaglandins 49:269-283.

Kemp JP (2003) Recent advances in the management of asthma using leukotriene modifiers. Am J Respir Med 2:139-156.

Kenakin T (1997) Differences between natural and recombinant G protein-coupled receptor systems with varying receptor/G protein stoichiometry. Trends Pharma col Sci 18:456-464.

Kenakin T (2002) Drug efficacy at G protein-coupled receptors. Annu Rev Pharmacol Toxicol 42:349-379.

Keppler D, Huber M, and Baumert T (1988) Leukotrienes as mediators in diseases of the liver. Semin Liver Dis 8:357-366.

Kihara Y, Yokomizo T, Kunita A, Morishita Y, Fukayama M, Ishii S, and Shimizu T (2010) The leukotriene B4 receptor, BLT1, is required for the induction of experimental autoimmune encephalomyelitis. Biochem Biophys Res Commun 394:673678

Kim GY, Lee JW, Cho SH, Seo JM, and Kim JH (2009) Role of the low-affinity 
leukotriene B4 receptor BLT2 in VEGF-induced angiogenesis. Arterioscler Thromb Vasc Biol 29:915-920.

Kim HJ, Kim DK, Kim H, Koh JY, Kim KM, Noh MS, Lee S, Kim S, Park SH, Kim JJ, et al. (2008) Involvement of the BLT2 receptor in the itch-associated scratching induced by 12-(S)-lipoxygenase products in ICR mice. Br J Pharmacol 154:10731078.

Kim MH, Lee YJ, Kim MO, Kim JS, and Han HJ (2010) Effect of leukotriene D4 on mouse embryonic stem cell migration and proliferation: Involvement of PI3K/Akt as well as GSK-3 $\beta / \beta$-catenin signaling pathways. J Cell Biochem 111:686-698.

Kim N and Luster AD (2007) Regulation of immune cells by eicosanoid receptors. ScientificWorldJournal 7:1307-1328.

Kim ND, Chou RC, Seung E, Tager AM, and Luster AD (2006a) A unique requirement for the leukotriene B4 receptor BLT1 for neutrophil recruitment in inflammatory arthritis. J Exp Med 203:829-835.

Kim SH, Oh JM, Kim YS, Palmer LJ, Suh CH, Nahm DH, and Park HS (2006b) Cysteinyl leukotriene receptor 1 promoter polymorphism is associated with aspirin-intolerant asthma in males. Clin Exp Allergy 36:433-439.

Kim SH, Yang EM, Park HJ, Ye YM, Lee HY, and Park HS (2007a) Differential contribution of the CysLTR1 gene in patients with aspirin hypersensitivity. J Clin Immunol 27:613-619.

Kim SH, Ye YM, Hur GY, Lee SK, Sampson AP, Lee HY, and Park HS (2007b) CysLTR1 promoter polymorphism and requirement for leukotriene receptor antagonist in aspirin-intolerant asthma patients. Pharmacogenomics 8:1143-1150.

Kimura K, Noguchi E, Shibasaki M, Arinami T, Yokouchi Y, Takeda K, YamakawaKobayashi K, Matsui A, and Hamaguchi H (1999) Linkage and association of atopic asthma to markers on chromosome 13 in the Japanese population. Hum Mol Genet 8:1487-1490

Kitaura J, Kinoshita T, Matsumoto M, Chung S, Kawakami Y, Leitges M, Wu D, Lowell CA, and Kawakami T (2005) IgE - and IgE+Ag-mediated mast cell migration in an autocrine/paracrine fashion. Blood 105:3222-3229.

Kiwamoto T, Ishii Y, Morishima Y, Yoh K, Kikuchi N, Haraguchi N, Masuko H, Kawaguchi M, Nomura A, Sakamoto T, et al. (2011) Blockade of cysteinyl leukotriene-1 receptors suppresses airway remodelling in mice overexpressing GATA-3. Clin Exp Allergy 41:116-128.

Kjeldsen L, Bjerrum OW, Askaa J, and Borregaard N (1992) Subcellular localization and release of human neutrophil gelatinase, confirming the existence of separate gelatinase-containing granules. Biochem $J$ 287:603-610.

Klotsman M, York TP, Pillai SG, Vargas-Irwin C, Sharma SS, van den Oord EJ, and Anderson WH (2007) Pharmacogenetics of the 5-lipoxygenase biosynthetic pathway and variable clinical response to montelukast. Pharmacogenet Genomics 17:189-196.

Kondo K, Umemura K, Ohmura T, Hashimoto H, and Nakashima M (1998) Suppression of intimal hyperplasia by a 5-lipoxygenase inhibitor, MK-886: studies with a photochemical model of endothelial injury. Thromb Haemost 79:635-639.

Kopriva F, Janostáková A, Jarmila S, Zápalka M, and Hajduch M (2006) Montelukast decreases plasma endothelin-1 and serum eosinophil cationic protein levels in paediatric atopic asthma. Clin Drug Investig 26:351-356.

Krey G, Braissant O, L'Horset F, Kalkhoven E, Perroud M, Parker MG, and Wahli W (1997) Fatty acids, eicosanoids, and hypolipidemic agents identified as ligands of peroxisome proliferator-activated receptors by coactivator-dependent receptor ligand assay. Mol Endocrinol 11:779-791.

Kristo F, Hardy GJ, Anderson TJ, Sinha S, Ahluwalia N, Lin AY, Passeri J, ScherrerCrosbie M, and Gerszten RE (2010) Pharmacological inhibition of BLT1 diminishes early abdominal aneurysm formation. Atherosclerosis 210:107-113.

Kroeze WK, Sheffler DJ, and Roth BL (2003) G-protein-coupled receptors at a glance. J Cell Sci 116:4867-4869.

Kumasawa F, Hashimoto S, Onose A, Jibiki I, Mizumura K, Matsumoto K, Maruoka S, Gon Y, Kobayashi T, Takahashi N, et al. (2005) Apoptosis signal-regulating kinase 1 in leukotriene $\mathrm{D}_{4}$-induced activator protein-1 activation in airway smooth muscle cells. Eur J Pharmacol 517:11-16.

Kuniyeda K, Okuno T, Terawaki K, Miyano M, Yokomizo T, and Shimizu T (2007) Identification of the intracellular region of the leukotriene $\mathrm{B}_{4}$ receptor type 1 that is specifically involved in $\mathrm{G}_{i}$ activation. J Biol Chem 282:3998-4006.

Kurowski M, Kuna P, and Górski P (2004) Montelukast plus cetirizine in the prophylactic treatment of seasonal allergic rhinitis: influence on clinical symptoms and nasal allergic inflammation. Allergy 59:280-288

Kushiya M, Saito K, Kikuchi I, Kobayashi T, Hagiwara K, Kanazawa M, and Nagata M (2006) Differential effects of salbutamol and montelukast on eosinophil adhesion and superoxide anion generation. Int Arch Allergy Immunol 140 (Suppl 1):17-22.

Labat C, Ortiz JL, Norel X, Gorenne I, Verley J, Abram TS, Cuthbert NJ, Tudhope SR, Norman P, and Gardiner P (1992) A second cysteinyl leukotriene receptor in human lung. J Pharmacol Exp Ther 263:800-805.

Lagos JA and Marshall GD (2007) Montelukast in the management of allergic rhinitis. Ther Clin Risk Manag 3:327-332.

Laitinen A, Lindqvist A, Halme M, Altraja A, and Laitinen LA (2005) Leukotriene $\mathrm{E}_{4}$-induced persistent eosinophilia and airway obstruction are reversed by zafirlukast in patients with asthma. J Allergy Clin Immunol 115:259-265.

Lamoureux J, Stankova J, and Rola-Pleszczynski M (2006) Leukotriene $\mathrm{D}_{4}$ enhances immunoglobulin production in CD40-activated human B lymphocytes. J Allergy Clin Immunol 117:924-930.

Langlois A, Ferland C, Tremblay GM, and Laviolette M (2006) Montelukast regulates eosinophil protease activity through a leukotriene-independent mechanism. $J$ Allergy Clin Immunol 118:113-119.

Langmack EL and Martin RJ (2010) Heterogeneity of response to asthma controller therapy: clinical implications. Curr Opin Pulm Med 16:13-18.

Larré S, Tran N, Fan C, Hamadeh H, Champigneulles J, Azzouzi R, Cussenot O, Mangin P, and Olivier JL (2008) PGE2 and LTB4 tissue levels in benign and cancerous prostates. Prostaglandins Other Lipid Mediat 87:14-19.

Lecca D, Trincavelli ML, Gelosa P, Sironi L, Ciana P, Fumagalli M, Villa G, Verderio C, Grumelli C, Guerrini U, et al. (2008) The recently identified P2Y-like receptor
GPR17 is a sensor of brain damage and a new target for brain repair. PLoS One 3:e3579.

Lee DK, Haggart K, Robb FM, and Lipworth BJ (2004a) Montelukast protects against nasal lysine-aspirin challenge in patients with aspirin-induced asthma Eur Respir J 24:226-230.

Lee DK, Jackson CM, Soutar PC, Fardon TC, and Lipworth BJ (2004b) Effects of single or combined histamine H1-receptor and leukotriene CysLT1-receptor antagonism on nasal adenosine monophosphate challenge in persistent allergic rhinitis. Br J Clin Pharmacol 57:714-719.

Lee KS, Kim SR, Park HS, Park SJ, Min KH, Lee KY, Jin SM, and Lee YC (2007a) Cysteinyl leukotriene upregulates IL-11 expression in allergic airway disease of mice. J Allergy Clin Immunol 119:141-149.

Lee SY, Kim HB, Kim JH, Kim BS, Kang MJ, Jang SO, Seo HJ, and Hong SJ (2007b) Responsiveness to montelukast is associated with bronchial hyperresponsivenes and total immunoglobulin $\mathrm{E}$ but not polymorphisms in the leukotriene $\mathrm{C} 4$ synthase and cysteinyl leukotriene receptor 1 genes in Korean children with exerciseinduced asthma (EIA). Clin Exp Allergy 37:1487-1493.

Lee TH, Austen KF, Corey EJ, and Drazen JM (1984) Leukotriene $\mathrm{E}_{4}$-induced airway hyperresponsiveness of guinea pig tracheal smooth muscle to histamine and evidence for three separate sulfidopeptide leukotriene receptors. Proc Nat Acad Sci USA 81:4922-4925.

Leikauf GD, Claesson HE, Doupnik CA, Hybbinette S, and Grafström RC (1990) Cysteinyl leukotrienes enhance growth of human airway epithelial cells. Am J Physiol 259:L255-L261.

Leonardi S, Marchese G, Marseglia GL, and La Rosa M (2007) Montelukast in allergic diseases beyond asthma. Allergy Asthma Proc 28:287-291.

Leppert D, Hauser SL, Kishiyama JL, An S, Zeng L, and Goetzl EJ (1995) Stimulation of matrix metalloproteinase-dependent migration of $\mathrm{T}$ cells by eicosanoids. FASEB J 9:1473-1481.

Lewis RA, Drazen JM, Austen KF, Clark DA, and Corey EJ (1980) Identification of the $\mathrm{C}_{6}$-S-conjugate of leukotriene $\mathrm{A}$ with cysteine as a naturally occurring slow reacting substance of anaphylaxis (SRS-A). Importance of the 11-cis-geometry for biological activity. Biochem Biophys Res Commun 96:271-277.

Li AM, Abdullah VJ, Tsen CS, Au CT, Lam HS, So HK, Chan MH, Leung AW, Chan IH, Lam CW, et al. (2009) Leukotriene receptor antagonist in the treatment of childhood allergic rhinitis-a randomized placebo-controlled study. Pediatr Pulmonol 44:1085-1092.

Liao T, Ke Y, Shao WH, Haribabu B, Kaplan HJ, Sun D, and Shao H (2006) Blockade of the interaction of leukotriene b4 with its receptor prevents development of autoimmune uveitis. Invest Ophthalmol Vis Sci 47:1543-1549.

Lima JJ, Zhang S, Grant A, Shao L, Tantisira KG, Allayee H, Wang J, Sylvester J, Holbrook J, Wise R, et al. (2006) Influence of leukotriene pathway polymorphisms on response to montelukast in asthma. Am J Respir Crit Care Med 173:379-385.

Lin Q, Ruuska SE, Shaw NS, Dong D, and Noy N (1999) Ligand selectivity of the peroxisome proliferator-activated receptor $\alpha$. Biochemistry 38:185-190.

Lindström P, Lerner R, Palmblad J, and Patarroyo M (1990) Rapid adhesive responses of endothelial cells and of neutrophils induced by leukotriene B4 are mediated by leucocytic adhesion protein CD18. Scand J Immunol 31:737-744.

Lipworth BJ, Dempsey OJ, Aziz I, and Wilson AM (2000) Effects of adding a leukotriene antagonist or a long-acting $\beta_{2}$-agonist in asthmatic patients with the glycine-16 $\beta_{2}$-adrenoceptor genotype. Am J Med 109:114-121.

Liu A, Claesson HE, Mahshid Y, Klein G, and Klein E (2008) Leukotriene B $_{4}$ activates $\mathrm{T}$ cells that inhibit B-cell proliferation in EBV-infected cord bloodderived mononuclear cell cultures. Blood 111:2693-2703.

Liu AH (2000) Allergy and asthma: classic TH2 diseases (?). Allergy Asthma Proc 21:227-230.

Lötzer K, Funk CD, and Habenicht AJ (2005) The 5-lipoxygenase pathway in arterial wall biology and atherosclerosis. Biochim Biophys Acta 1736:30-37.

Lötzer K, Spanbroek R, Hildner M, Urbach A, Heller R, Bretschneider E, Galczenski H, Evans JF, and Habenicht AJ (2003) Differential leukotriene receptor expression and calcium responses in endothelial cells and macrophages indicate 5-lipoxygenase-dependent circuits of inflammation and atherogenesis. Arterioscler Thromb Vasc Biol 23:e32-e36.

Lundeen KA, Sun B, Karlsson L, and Fourie AM (2006) Leukotriene B $_{4}$ receptors BLT1 and BLT2: expression and function in human and murine mast cells. J Immunol 177:3439-3447.

Lynch KR, O’Neill GP, Liu Q, Im DS, Sawyer N, Metters KM, Coulombe N, Abramovitz M, Figueroa DJ, Zeng Z, et al. (1999) Characterization of the human cysteinyl leukotriene $\mathrm{CysLT}_{1}$ receptor. Nature 399:789-793.

Maeba S, Ichiyama T, Ueno Y, Makata H, Matsubara T, and Furukawa S (2005) Effect of montelukast on nuclear factor $\kappa \mathrm{B}$ activation and proinflammatory molecules. Ann Allergy Asthma Immunol 94:670-674.

Maekawa A, Austen KF, and Kanaoka Y (2002) Targeted gene disruption reveals the role of cysteinyl leukotriene 1 receptor in the enhanced vascular permeability of mice undergoing acute inflammatory responses. J Biol Chem 277:20820-20824.

Maekawa A, Balestrieri B, Austen KF, and Kanaoka Y (2009) GPR17 is a negative regulator of the cysteinyl leukotriene 1 receptor response to leukotriene D4. Proc Natl Acad Sci USA 106:11685-11690.

Maekawa A, Kanaoka Y, Lam BK, and Austen KF (2001) Identification in mice of two isoforms of the cysteinyl leukotriene 1 receptor that result from alternative splicing. Proc Natl Acad Sci USA 98:2256-2261.

Maekawa A, Kanaoka Y, Xing W, and Austen KF (2008) Functional recognition of a distinct receptor preferential for leukotriene $\mathrm{E}_{4}$ in mice lacking the cysteinyl leukotriene 1 and 2 receptors. Proc Natl Acad Sci USA 105:16695-16700.

Maekawa A, Xing W, Austen KF, and Kanaoka Y (2010) GPR17 regulates immune pulmonary inflammation induced by house dust mites. J Immunol 185:18461854.

Magnusson C, Ehrnström R, Olsen J, and Sjölander A (2007) An increased expression of cysteinyl leukotriene 2 receptor in colorectal adenocarcinomas correlate with high differentiation. Cancer Res 67:9190-9198.

Magnusson C, Liu J, Ehrnström R, Manjer J, Jirström K, Andersson T, and Sjö- 
lander A (2011) Cysteinyl leukotriene receptor expression pattern affects migration of breast cancer cells and survival of breast cancer patients. Int $J$ Cancer 129:9-22.

Magnusson C, Mezhybovska M, Lörinc E, Fernebro E, Nilbert M, and Sjölander A (2010) Low expression of CysLT1R and high expression of CysLT2R mediate good prognosis in colorectal cancer. Eur J Cancer 46:826-835.

Malmsten CL, Palmblad J, Udén AM, Rådmark O, Engstedt L, and Samuelsson B (1980) Leukotriene B4: a highly potent and stereospecific factor stimulating migration of polymorphonuclear leukocytes. Acta Physiol Scand 110:449-451.

Mamedova L, Capra V, Accomazzo MR, Gao ZG, Ferrario S, Fumagalli M, Abbracchio MP, Rovati GE, and Jacobson KA (2005) CysLT 1 leukotriene receptor antagonists inhibit the effects of nucleotides acting at P2Y receptors. Biochem Pharmacol 71:115-125.

Mancuso P and Peters-Golden M (2000) Modulation of alveolar macrophage phagocytosis by leukotrienes is Fc receptor-mediated and protein kinase C-dependent. Am J Respir Cell Mol Biol 23:727-733.

Marinissen MJ and Gutkind JS (2001) G-protein-coupled receptors and signaling networks: emerging paradigms. Trends Pharmacol Sci 22:368-376.

Marrache AM, Gobeil F, Zhu T, and Chemtob S (2005) Intracellular signaling of lipid mediators via cognate nuclear G protein-coupled receptors. Endothelium 12:63-72.

Martin TR, Gerard NP, Galli SJ, and Drazen JM (1988) Pulmonary responses to bronchoconstrictor agonists in the mouse. J Appl Physiol 64:2318-2323.

Martin V, Sawyer N, Stocco R, Unett D, Lerner MR, Abramovitz M, and Funk CD (2001) Molecular cloning and functional characterization of murine cysteinylleukotriene $1\left(\right.$ CysLT $\left._{1}\right)$ receptors. Biochem Pharmacol 62:1193-1200.

Massoumi R, Larsson C, and Sjölander A (2002) Leukotriene $\mathrm{D}_{4}$ induces stress-fibre formation in intestinal epithelial cells via activation of RhoA and PKCdelta. J Cell Sci 115:3509-3515.

Massoumi R, Nielsen CK, Azemovic D, and Sjölander A (2003) Leukotriene $\mathrm{D}_{4}$ induced adhesion of Caco-2 cells is mediated by prostaglandin $\mathrm{E}_{2}$ and upregulation of $\alpha 2 \beta 1$-integrin. Exp Cell Res 289:342-351.

Massoumi R and Sjölander A (1998) The inflammatory mediator leukotriene D4 triggers a rapid reorganisation of the actin cytoskeleton in human intestinal epithelial cells. Eur J Cell Biol 76:185-191.

Massoumi R and Sjölander A (2001) Leukotriene $\mathrm{D}_{4}$ affects localisation of vinculin in intestinal epithelial cells via distinct tyrosine kinase and protein kinase C controlled events. $J$ Cell Sci 114:1925-1934.

Mastalerz L, Gawlewicz-Mroczka A, Nizankowska E, Cmiel A, and Szczeklik A (2002a) Protection against exercise-induced bronchoconstriction by montelukast in aspirin-sensitive and aspirin-tolerant patients with asthma. Clin Exp Allergy 32:1360-1365.

Mastalerz L, Nizankowska E, Sanak M, Mejza F, Pierzchalska M, Bazan-Socha S, Bestynska-Krypel A, Cmiel A, and Szczeklik A (2002b) Clinical and genetic features underlying the response of patients with bronchial asthma to treatment with a leukotriene receptor antagonist. Eur J Clin Invest 32:949-955.

Masuda K, Yokomizo T, Izumi T, and Shimizu T (1999) cDNA cloning and characterization of guinea-pig leukotriene $\mathrm{B} 4$ receptor. Biochem $J$ 342:79-85.

Mathis SP, Jala VR, Lee DM, and Haribabu B (2010) Nonredundant roles for leukotriene $\mathrm{B}_{4}$ receptors BLT1 and BLT2 in inflammatory arthritis. J Immunol 185:3049-3056.

Matsuyama M, Hayama T, Funao K, Kawahito Y, Sano H, Takemoto Y, Nakatani T, and Yoshimura R (2007) Overexpression of cysteinyl LT1 receptor in prostate cancer and CysLT1R antagonist inhibits prostate cancer cell growth through apoptosis. Oncol Rep 18:99-104.

Mattern MR, Mong S, Mong SM, Bartus JO, Sarau HM, Clark MA, Foley JJ, and Crooke ST (1990) Transient activation of topoisomerase I in leukotriene D4 signal transduction in human cells. Biochem J 265:101-107.

McHugh D, McMaster RS, Pertwee RG, Roy S, Mahadevan A, Razdan RK, and Ross RA (2006) Novel compounds that interact with both leukotriene $\mathrm{B}_{4}$ receptors and vanilloid TRPV1 receptors. J Pharmacol Exp Ther 316:955-965.

McIntyre TM, Zimmerman GA, and Prescott SM (1986) Leukotrienes $\mathrm{C}_{4}$ and $\mathrm{D}_{4}$ stimulate human endothelial cells to synthesize platelet-activating factor and bind neutrophils. Proc Natl Acad Sci USA 83:2204-2208.

McMahon B, Mitchell D, Shattock R, Martin F, Brady HR, and Godson C (2002) Lipoxin, leukotriene, and PDGF receptors cross-talk to regulate mesangial cell proliferation. FASEB $J$ 16:1817-1819.

McMahon B, Stenson C, McPhillips F, Fanning A, Brady HR, and Godson C (2000) Lipoxin $\mathrm{A}_{4}$ antagonizes the mitogenic effects of leukotriene $\mathrm{D}_{4}$ in human renal mesangial cells. Differential activation of MAP kinases through distinct receptors. $J$ Biol Chem 275:27566-27575.

Mechiche H, Candenas L, Pinto FM, Nazeyrollas P, Clément C, and Devillier P 2004) Characterization of cysteinyl leukotriene receptors on human saphenous veins: antagonist activity of montelukast and its metabolites. J Cardiovasc Pharmacol 43:113-120.

Medoff BD, Seung E, Wain JC, Means TK, Campanella GS, Islam SA, Thomas SY, Ginns LC, Grabie N, Lichtman AH, et al. (2005) BLT1-mediated T cell trafficking is critical for rejection and obliterative bronchiolitis after lung transplantation. $J$ Exp Med 202:97-110.

Meliton AY, Munoz NM, and Leff AR (2007) Blockade of avidity and focal clustering of $\beta_{2}$-integrin by cysteinyl leukotriene antagonism attenuates eosinophil adhesion. $J$ Allergy Clin Immunol 120:1316-1323.

Mellor EA, Austen KF, and Boyce JA (2002) Cysteinyl leukotrienes and uridine diphosphate induce cytokine generation by human mast cells through an interleukin 4-regulated pathway that is inhibited by leukotriene receptor antagonists. $J$ Exp Med 195:583-592.

Mellor EA, Frank N, Soler D, Hodge MR, Lora JM, Austen KF, and Boyce JA (2003) Expression of the type 2 receptor for cysteinyl leukotrienes (CysLT2R) by human mast cells: functional distinction from CysLT1R. Proc Natl Acad Sci USA 100: 11589-11593.

Mellor EA, Maekawa A, Austen KF, and Boyce JA (2001) Cysteinyl leukotriene receptor 1 is also a pyrimidinergic receptor and is expressed by human mast cells Proc Natl Acad Sci USA 98:7964-7969.

Meltzer EO, Malmstrom K, Lu S, Prenner BM, Wei LX, Weinstein SF, Wolfe JD, an Reiss TF (2000) Concomitant montelukast and loratadine as treatment for seasonal allergic rhinitis: a randomized, placebo-controlled clinical trial. J Allergy Clin Immunol 105:917-922.

Mesnier D and Banères JL (2004) Cooperative conformational changes in a G-protein-coupled receptor dimer, the leukotriene $\mathrm{B}_{4}$ receptor BLT1. J Biol Chem 279:49664-49670

Mezhybovska M, Wikström K, Ohd JF, and Sjölander A (2006) The inflammatory mediator leukotriene $\mathrm{D}_{4}$ induces $\beta$-catenin signaling and its association with antiapoptotic Bcl-2 in intestinal epithelial cells. J Biol Chem 281:6776-6784.

Michalik L, Auwerx J, Berger JP, Chatterjee VK, Glass CK, Gonzalez FJ, Grimald PA, Kadowaki T, Lazar MA, O'Rahilly S, et al. (2006) International Union of Pharmacology. LXI. Peroxisome proliferator-activated receptors. Pharmacol Rev 58:726-741.

Micheletto C, Tognella S, Visconti M, Pomari C, Trevisan F, and Dal Negro RW (2004) Montelukast $10 \mathrm{mg}$ improves nasal function and nasal response to aspirin in ASA-sensitive asthmatics: a controlled study vs placebo. Allergy 59:289-294.

Micheletto C, Visconti M, Tognella S, Facchini FM, and Dal Negro RW (2006) Aspirin induced asthma (AIA) with nasal polyps has the highest basal LTE4 excretion: a study vs AIA without polyps, mild topic asthma, and normal controls. Eur Ann Allergy Clin Immunol 38:20-23.

Milanese M, Riccio AM, Gamalero C, De Giovanni B, Brichetto L, Baroffio M, Crimi $\mathrm{E}$, Brusasco V, and Canonica GW (2005) A model of allergen-driven human airway contraction: $\beta_{2}$ pathway dysfunction without cytokine involvement. Ann Allergy Asthma Immunol 94:273-278.

Milligan G (2009) G protein-coupled receptor hetero-dimerization: contribution to pharmacology and function. $\mathrm{Br} J$ Pharmacol 158:5-14.

Minciullo PL, Saija A, Bonanno D, Ferlazzo E, and Gangemi S (2004) Montelukastinduced generalized urticaria. Ann Pharmacother 38:999-1001.

Mirshafiey A and Jadidi-Niaragh F (2010) Immunopharmacological role of the leukotriene receptor antagonists and inhibitors of leukotrienes generating enzymes in multiple sclerosis. Immunopharmacol Immunotoxicol 32:219-227.

Mita H, Endoh S, Kudoh M, Kawagishi Y, Kobayashi M, Taniguchi M, and Akiyama $\mathrm{K}$ (2001a) Possible involvement of mast-cell activation in aspirin provocation of aspirin-induced asthma. Allergy 56:1061-1067.

Mita H, Hasegawa M, Saito H, and Akiyama K (2001b) Levels of cysteinyl leukotriene receptor mRNA in human peripheral leucocytes: significantly higher expression of cysteinyl leukotriene receptor 2 mRNA in eosinophils. Clin Exp Allergy 31:1714-1723.

Miyahara N, Ohnishi H, Matsuda H, Miyahara S, Takeda K, Koya T, Matsubara S, Okamoto M, Dakhama A, Haribabu B, et al. (2008) Leukotriene $\mathrm{B}_{4}$ receptor expression on dendritic cells is required for the development of Th2 responses and allergen-induced airway hyperresponsiveness. J Immunol 181:1170-1178.

Miyahara N, Takeda K, Miyahara S, Matsubara S, Koya T, Joetham A, Krishnan E, Dakhama A, Haribabu B, and Gelfand EW (2005a) Requirement for leukotriene B receptor 1 in allergen-induced airway hyperresponsiveness. Am J Respir Crit Care Med 172:161-167.

Miyahara N, Takeda K, Miyahara S, Taube C, Joetham A, Koya T, Matsubara S, Dakhama A, Tager AM, Luster AD, et al. (2005b) Leukotriene $\mathrm{B}_{4}$ receptor-1 is essential for allergen-mediated recruitment of $\mathrm{CD}^{+} \mathrm{T}$ cells and airway hyperresponsiveness. J Immunol 174:4979-4984.

Mohle R, Boehmler AM, Denzlinger C, and Kanz L (2003) Nonpeptide mediators in the hematopoietic microenvironment. Ann NY Acad Sci 996:61-66.

Mollerup J, Eriksen HN, Albertsen J, Wulff T, Lambert IH, and Hoffmann EK (2007) Homologous desensitisation of the mouse leukotriene $\mathrm{B}_{4}$ receptor involves protein kinase C-mediated phosphorylation of serine 127. Cell Physiol Biochem 20:143156.

Montuschi P, Kharitonov SA, Ciabattoni G, and Barnes PJ (2003) Exhaled leukotrienes and prostaglandins in COPD. Thorax 58:585-588

Montuschi P, Sala A, Dahlén SE, and Folco G (2007) Pharmacological modulation of the leukotriene pathway in allergic airway disease. Drug Discov Today 12:404412

Moos MP and Funk CD (2008) Endothelial cysteinyl leukotriene 2 receptor expression and myocardial ischemia/reperfusion injury. Trends Cardiovasc Med 18:268 273.

Moos MP, Mewburn JD, Kan FW, Ishii S, Abe M, Sakimura K, Noguchi K, Shimizu T, and Funk CD (2008) Cysteinyl leukotriene 2 receptor-mediated vascular permeability via transendothelial vesicle transport. FASEB J 22:4352-4362.

Moraes J, Assreuy J, Canetti C, and Barja-Fidalgo C (2010) Leukotriene $\mathrm{B}_{4}$ mediates vascular smooth muscle cell migration through $\alpha \mathrm{v} \beta 3$ integrin transactivation. Atherosclerosis 212:406-413.

Mueller CF, Wassmann K, Widder JD, Wassmann S, Chen CH, Keuler B, Kudin A Kunz WS, and Nickenig G (2008) Multidrug resistance protein-1 affects oxidative stress, endothelial dysfunction, and atherogenesis via leukotriene $\mathrm{C}_{4}$ export. $\mathrm{Cir}$ culation 117:2912-2918.

Murray J, Ward C, O'Flaherty JT, Dransfield I, Haslett C, Chilvers ER, and Ross AG (2003) Role of leukotrienes in the regulation of human granulocyte behaviour dissociation between agonist-induced activation and retardation of apoptosis. $\mathrm{Br}$ Pharmacol 139:388-398.

Nabe T, Yamashita K, Miura M, Kawai T, and Kohno S (2002) Cysteinyl leukotrienedependent interleukin-5 production leading to eosinophilia during late asthmatic response in guinea-pigs. Clin Exp Allergy 32:633-640.

Nag S, Lamkhioued B, and Renzi PM (2002) Interleukin-2-induced increased airway responsiveness and lung Th2 cytokine expression occur after antigen challenge through the leukotriene pathway. Am J Respir Crit Care Med 165:1540-1545.

Nagata M and Saito K (2003) The roles of cysteinyl leukotrienes in eosinophilic inflammation of asthmatic airways. Int Arch Allergy Immunol 131 (Suppl 1):7-10.

Nagata M, Saito K, Kikuchi I, Hagiwara K, and Kanazawa M (2005) Effect of the 
cysteinyl leukotriene antagonist pranlukast on transendothelial migration of eosinophils. Int Arch Allergy Immunol 137 (Suppl 1):2-6.

Nagata M, Saito K, Tsuchiya K, and Sakamoto Y (2002) Leukotriene $\mathrm{D}_{4}$ upregulates eosinophil adhesion via the cysteinyl leukotriene 1 receptor. J Allergy Clin Immunol 109:676-680.

Nagy E, Andersson DC, Caidahl K, Eriksson MJ, Eriksson P, Franco-Cereceda A, Hansson GK, and Bäck M (2011) Upregulation of the 5-lipoxygenase pathway in human aortic valves correlates with severity of stenosis and leads to leukotrieneinduced effects on valvular myofibroblasts. Circulation 123:1316-1325.

Naik S, Billington CK, Pascual RM, Deshpande DA, Stefano FP, Kohout TA, Eckman DM, Benovic JL, and Penn RB (2005) Regulation of cysteinyl leukotriene type 1 receptor internalization and signaling. J Biol Chem 280:8722-8732.

Nannini LJ Jr and Flores DM (2003) Bronchodilator effect of zafirlukast in subjects with chronic obstructive pulmonary disease. Pulm Pharmacol Ther 16:307-311.

Narala VR, Adapala RK, Suresh MV, Brock TG, Peters-Golden M, and Reddy RC (2010) Leukotriene $B_{4}$ is a physiologically relevant endogenous peroxisome proliferator-activated receptor- $\alpha$ agonist. J Biol Chem 285:22067-22074.

Nathan RA, Bernstein JA, Bielory L, Bonuccelli CM, Calhoun WJ, Galant SP, Hanby LA, Kemp JP, Kylstra JW, Nayak AS, et al. (1998) Zafirlukast improves asthma symptoms and quality of life in patients with moderate reversible airflow obstruction. J Allergy Clin Immunol 102:935-942.

Nayak A (2004) A review of montelukast in the treatment of asthma and allergic rhinitis. Expert Opin Pharmacother 5:679-686.

Nayak A and Langdon RB (2007) Montelukast in the treatment of allergic rhinitis: an evidence-based review. Drugs 67:887-901.

Nayak AS, Philip G, Lu S, Malice MP, Reiss TF, and Montelukast Fall Rhinitis Investigator Group (2002) Efficacy and tolerability of montelukast alone or in combination with loratadine in seasonal allergic rhinitis: a multicenter, randomized, double-blind, placebo-controlled trial performed in the fall. Ann Allergy Asthma Immunol 88:592-600.

Neu I, Mallinger J, Wildfeuer A, and Mehlber L (1992) Leukotrienes in the cerebrospinal fluid of multiple sclerosis patients. Acta Neurol Scand 86:586-587.

Neu IS, Metzger G, Zschocke J, Zelezny R, and Mayatepek E (2002) Leukotrienes in patients with clinically active multiple sclerosis. Acta Neurol Scand 105:63-66.

Neves JS, Radke AL, and Weller PF (2010) Cysteinyl leukotrienes acting via granule membrane-expressed receptors elicit secretion from within cell-free human eosinophil granules. J Allergy Clin Immunol 125:477-482.

Ng CF, Sun FF, Taylor BM, Wolin MS, and Wong PY (1991) Functional properties of guinea pig eosinophil leukotriene $\mathrm{B}_{4}$ receptor. J Immunol 147:3096-3103.

Nielsen CK, Campbell JI, Ohd JF, Mörgelin M, Riesbeck K, Landberg G, and Sjölander A (2005a) A novel localization of the G-protein-coupled CysLT1 receptor in the nucleus of colorectal adenocarcinoma cells. Cancer Res 65:732-742.

Nielsen CK, Massoumi R, Sonnerlind M, and Sjölander A (2005b) Leukotriene $\mathrm{D}_{4}$ activates distinct G-proteins in intestinal epithelial cells to regulate stress fibre formation and to generate intracellular $\mathrm{Ca}^{2+}$ mobilisation and ERK1/2 activation. Exp Cell Res 302:31-39.

Nieves D and Moreno JJ (2006) Role of 5-lipoxygenase pathway in the regulation of RAW 264.7 macrophage proliferation. Biochem Pharmacol 72:1022-1030.

Nonaka Y, Hiramoto T, and Fujita N (2005) Identification of endogenous surrogate ligands for human P2Y12 receptors by in silico and in vitro methods. Biochem Biophys Res Commun 337:281-288.

Noonan MJ, Chervinsky P, Brandon M, Zhang J, Kundu S, McBurney J, and Reiss TF (1998) Montelukast, a potent leukotriene receptor antagonist, causes doserelated improvements in chronic asthma. Montelukast Asthma Study Group. Eur Respir J 11:1232-1239.

Norel X and Brink C (2004) The quest for new cysteinyl-leukotriene and lipoxin receptors: recent clues. Pharmacol Ther 103:81-94

Nothacker HP, Wang Z, Zhu Y, Reinscheid RK, Lin SH, and Civelli O (2000) Molecular cloning and characterization of a second human cysteinyl leukotriene receptor: discovery of a subtype selective agonist. Mol Pharmacol 58:1601-1608.

O'Flaherty JT, Rossi AG, Redman JF, and Jacobson DP (1991) Tumor necrosis factor- $\alpha$ regulates expression of receptors for formyl-methionyl-leucyl-phenylalanine, leukotriene B4, and platelet-activating factor. Dissociation from priming in human polymorphonuclear neutrophils. J Immunol 147:3842-3847.

O'Sullivan S, Dahlén B, Dahlén SE, and Kumlin M (1996) Increased urinary excretion of the prostaglandin $\mathrm{D}_{2}$ metabolite $9 \alpha, 11 \beta$-prostaglandin $\mathrm{F}_{2}$ after aspirin challenge supports mast cell activation in aspirin-induced airway obstruction. $J$ Allergy Clin Immunol 98:421-432.

Obase Y, Matsuse H, Shimoda T, Haahtela T, and Kohno S (2005) Pathogenesis and management of aspirin-intolerant asthma. Treat Respir Med 4:325-336.

Obase Y, Shimoda T, Tomari SY, Mitsuta K, Kawano T, Matsuse H, and Kohno S (2002) Effects of pranlukast on chemical mediators in induced sputum on provocation tests in atopic and aspirin-intolerant asthmatic patients. Chest 121:143150 .

Ogasawara H, Ishii S, Yokomizo T, Kakinuma T, Komine M, Tamaki K, Shimizu T, and Izumi T (2002) Characterization of mouse cysteinyl leukotriene receptors mCysLT1 and mCysLT2: differential pharmacological properties and tissue distribution. J Biol Chem 277:18763-18768.

Ohd JF, Nielsen CK, Campbell J, Landberg G, Löfberg H, and Sjölander A (2003) Expression of the leukotriene $\mathrm{D}_{4}$ receptor CysLT1, COX-2, and other cell survival factors in colorectal adenocarcinomas. Gastroenterology 124:57-70.

Ohd JF, Wikström K, and Sjölander A (2000) Leukotrienes induce cell-survival signaling in intestinal epithelial cells. Gastroenterology 119:1007-1018.

Ohshima N, Nagase H, Koshino T, Miyamasu M, Yamaguchi M, Hirai K, Yamamoto K, Fujisawa T, Nakagawa N, Kishikawa K, et al. (2002) A functional study on CysLT $_{1}$ receptors in human eosinophils. Int Arch Allergy Immunol 129:67-75.

Okubo K and Baba K (2008) A double-blind non-inferiority clinical study of montelukast, a cysteinyl leukotriene receptor 1 antagonist, compared with pranlukast in patients with seasonal allergic rhinitis. Allergol Int 57:383-390.

Okuno T, Ago H, Terawaki K, Miyano M, Shimizu T, and Yokomizo T (2003) Helix
8 of the leukotriene $B_{4}$ receptor is required for the conformational change to the low affinity state after G-protein activation. J Biol Chem 278:41500-41509.

Okuno T, Iizuka Y, Okazaki H, Yokomizo T, Taguchi R, and Shimizu T (2008) $12(S)$-Hydroxyheptadeca- $5 Z, 8 E, 10 E$-trienoic acid is a natural ligand for leukotriene $\mathrm{B}_{4}$ receptor 2. J Exp Med 205:759-766.

Ortiz JL, Gorenne I, Cortijo J, Seller A, Labat C, Sarria B, Abram TS, Gardiner PJ, Morcillo E, and Brink C (1995) Leukotriene receptors on human pulmonary vascular endothelium. Br J Pharmacol 115:1382-1386.

Ortolani C, Mirone C, Fontana A, Folco GC, Miadonna A, Montalbetti N, Rinaldi M, Sala A, Tedeschi A, and Valente D (1987) Study of mediators of anaphylaxis in nasal wash fluids after aspirin and sodium metabisulfite nasal provocation in intolerant rhinitic patients. Ann Allergy 59:106-112.

Ott VL, Cambier JC, Kappler J, Marrack P, and Swanson BJ (2003) Mast celldependent migration of effector $\mathrm{CD}^{+} \mathrm{T}$ cells through production of leukotriene $\mathrm{B}_{4}$. Nat Immunol 4:974-981.

Øymar K and Aksnes L (2005) Increased levels of urinary leukotriene $\mathrm{E}_{4}$ in children with severe atopic eczema/dermatitis syndrome. Allergy 60:86-89.

Ozen Z, Mumbuc S, Sari I, Baglam T, Karatas E, and Kanlikama M (2007) Cysteinyl leukotriene receptor expression in aspirin-sensitive nasal polyposis patients. ORL $J$ Otorhinolaryngol Relat Spec 69:176-180.

Pacor ML, Di Lorenzo G, and Corrocher R (2001) Efficacy of leukotriene receptor antagonist in chronic urticaria. A double-blind, placebo-controlled comparison of treatment with montelukast and cetirizine in patients with chronic urticaria with intolerance to food additive and/or acetylsalicylic acid. Clin Exp Allergy 31:16071614

Palmblad J, Lerner R, and Larsson SH (1994) Signal transduction mechanisms for leukotriene $\mathrm{B}_{4}$ induced hyperadhesiveness of endothelial cells for neutrophils. I Immunol 152:262-269.

Palmblad J, Malmsten CL, Udén AM, Rådmark O, Engstedt L, and Samuelsson B (1981) Leukotriene $B_{4}$ is a potent and stereospecific stimulator of neutrophil chemotaxis and adherence. Blood 58:658-661.

Palmer LJ, Silverman ES, Weiss ST, and Drazen JM (2002) Pharmacogenetics of asthma. Am J Respir Crit Care Med 165:861-866.

Panettieri RA, Tan EM, Ciocca V, Luttmann MA, Leonard TB, and Hay DW (1998) Effects of $\mathrm{LTD}_{4}$ on human airway smooth muscle cell proliferation, matrix expression, and contraction in vitro: differential sensitivity to cysteinyl leukotriene receptor antagonists. Am J Respir Cell Mol Biol 19:453-461.

Papayianni A, Serhan CN, and Brady HR (1996) Lipoxin $\mathrm{A}_{4}$ and $\mathrm{B}_{4}$ inhibit leukotriene-stimulated interactions of human neutrophils and endothelial cells. J Immunol 156:2264-2272.

Parameswaran K, Watson R, Gauvreau GM, Sehmi R, and O'Byrne PM (2004) The effect of pranlukast on allergen-induced bone marrow eosinophilopoiesis in subjects with asthma. Am J Respir Crit Care Med 169:915-920.

Parhamifar L, Jeppsson B, and Sjölander A (2005) Activation of cPLA2 is required for leukotriene $\mathrm{D}_{4}$-induced proliferation in colon cancer cells. Carcinogenesis 26 1988-1998.

Parhamifar L, Sime W, Yudina Y, Vilhardt F, Mörgelin M, and Sjölander A (2010) A ligand-induced tyrosine phosphorylation of cysteinyl leukotriene receptor 1 triggers internalization and signaling in intestinal epithelial cells. PLoS One 5:e14439.

Park JS, Chang HS, Park CS, Lee JH, Lee YM, Choi JH, Park HS, Kim LH, Park BL, Choi YH, et al. (2005) Association analysis of cysteinyl-leukotriene receptor 2 (CYSLTR2) polymorphisms with aspirin intolerance in asthmatics. Pharmacogenet Genomics 15:483-492.

Park JS, Jang AS, Park SW, Lee YM, Uh ST, Kim YH, Cha JY, Park SM, and Park CS (2010) Protection of leukotriene receptor antagonist against aspirin-induced bronchospasm in asthmatics. Allergy Asthma Immunol Res 2:48-54.

Paruchuri S, Broom O, Dib K, and Sjölander A (2005) The pro-inflammatory mediator leukotriene $\mathrm{D}_{4}$ induces phosphatidylinositol 3-kinase and Rac-dependent migration of intestinal epithelial cells. $J$ Biol Chem 280:13538-13544.

Paruchuri S, Hallberg B, Juhas M, Larsson C, and Sjölander A (2002) Leukotriene $\mathrm{D}_{4}$ activates MAPK through a Ras-independent but PKC $\varepsilon$-dependent pathway in intestinal epithelial cells. J Cell Sci 115:1883-1893.

Paruchuri S, Jiang Y, Feng C, Francis SA, Plutzky J, and Boyce JA (2008) Leukotriene $\mathrm{E}_{4}$ activates peroxisome proliferator-activated receptor $\gamma$ and induces prostaglandin $\mathrm{D}_{2}$ generation by human mast cells. J Biol Chem 283:16477-16487.

Paruchuri S, Mezhybovska M, Juhas M, and Sjölander A (2006) Endogenous production of leukotriene $\mathrm{D}_{4}$ mediates autocrine survival and proliferation via CysLT receptor signalling in intestinal epithelial cells. Oncogene 25:6660-6665.

Paruchuri S and Sjölander A (2003) Leukotriene $\mathrm{D}_{4}$ mediates survival and proliferation via separate but parallel pathways in the human intestinal epithelial cell line Int 407. J Biol Chem 278:45577-45585.

Paruchuri S, Tashimo H, Feng C, Maekawa A, Xing W, Jiang Y, Kanaoka Y, Conley $\mathrm{P}$, and Boyce JA (2009) Leukotriene $\mathrm{E}_{4}$-induced pulmonary inflammation is mediated by the P2Y12 receptor. J Exp Med 206:2543-2555.

Patry C, Müller E, Laporte J, Rola-Pleszczynski M, Sirois P, and de Brum-Fernandes AJ (1996) Leukotriene receptors in HL-60 cells differentiated into eosinophils, monocytes and neutrophils. Prostaglandins Leukot Essent Fatty Acids 54:361-370.

Payan DG, Missirian-Bastian A, and Goetzl EJ (1984) Human T-lymphocyte subset specificity of the regulatory effects of leukotriene B4. Proc Natl Acad Sci USA 81:3501-3505.

Pedersen KE, Bochner BS, and Undem BJ (1997) Cysteinyl leukotrienes induce P-selectin expression in human endothelial cells via a non-CysLT1 receptormediated mechanism. J Pharmacol Exp Ther 281:655-662.

Peres CM, Aronoff DM, Serezani CH, Flamand N, Faccioli LH, and Peters-Golden M (2007) Specific leukotriene receptors couple to distinct G proteins to effect stimulation of alveolar macrophage host defense functions. J Immunol 179:5454-5461.

Pérez-Novo CA, Claeys C, Van Cauwenberge P, and Bachert C (2006) Expression of eicosanoid receptors subtypes and eosinophilic inflammation: implication on chronic rhinosinusitis. Respir Res 7:75.

Perng DW, Wu YC, Chang KT, Wu MT, Chiou YC, Su KC, Perng RP, and Lee YC 
(2006) Leukotriene C4 induces TGF- $\beta 1$ production in airway epithelium via p38 kinase pathway. Am J Respir Cell Mol Biol 34:101-107.

Peters-Golden M, Gleason MM, and Togias A (2006) Cysteinyl leukotrienes: multifunctional mediators in allergic rhinitis. Clin Exp Allergy 36:689-703.

Peters-Golden M and Henderson WR Jr (2005) The role of leukotrienes in allergic rhinitis. Ann Allergy Asthma Immunol 94:609-618; quiz 618-620, 669.

Peters-Golden M and Henderson WR Jr (2007) Leukotrienes. N Engl J Med 357: 1841-1854.

Pétrin D, Turcotte S, Gilbert AK, Rola-Pleszczynski M, and Stankova J (2006) The anti-apoptotic effect of leukotriene B4 in neutrophils: a role for phosphatidylinositol 3-kinase, extracellular signal-regulated kinase and Mcl-1. Cell Signal 18: $479-487$

Pettersson A, Richter J, and Owman C (2003) Flow cytometric mapping of the leukotriene B4 receptor, BLT1, in human bone marrow and peripheral blood using specific monoclonal antibodies. Int Immunopharmacol 3:1467-1475.

Pettersson A, Sabirsh A, Bristulf J, Kidd-Ljunggren K, Ljungberg B, Owman C, and Karlsson U (2005) Pro- and anti-inflammatory substances modulate expression of the leukotriene B4 receptor, BLT1, in human monocytes. J Leukoc Biol 77:10181025

Phan H, Moeller ML, and Nahata MC (2009) Treatment of allergic rhinitis in infants and children: efficacy and safety of second-generation antihistamines and the leukotriene receptor antagonist montelukast. Drugs 69:2541-2576.

Philip G, Nayak AS, Berger WE, Leynadier F, Vrijens F, Dass SB, and Reiss TF (2004) The effect of montelukast on rhinitis symptoms in patients with asthma and easonal allergic rhinitis. Curr Med Res Opin 20:1549-1558.

Piatti G, Ceriotti L, Cavallaro G, Ambrosetti U, Mantovani M, Pistone A, and Centanni S (2003) Effects of zafirlukast on bronchial asthma and allergic rhinitis. Pharmacol Res 47:541-547.

Picado C, Ramis I, Rosellò J, Prat J, Bulbena O, Plaza V, Montserrat JM, and Gelpí E (1992) Release of peptide leukotriene into nasal secretions after local instillation of aspirin in aspirin-sensitive asthmatic patients. Am Rev Respir Dis 145:65-69.

Pillai SG, Cousens DJ, Barnes AA, Buckley PT, Chiano MN, Hosking LK, Cameron LA, Fling ME, Foley JJ, Green A, et al. (2004) A coding polymorphism in the CYSLT2 receptor with reduced affinity to LTD4 is associated with asthma. Phar macogenetics 14:627-633.

Pizzichini E, Leff JA, Reiss TF, Hendeles L, Boulet LP, Wei LX, Efthimiadis AE, Zhang J, and Hargreave FE (1999) Montelukast reduces airway eosinophilic inflammation in asthma: a randomized, controlled trial. Eur Respir $J$ 14:12-18.

Pollock K and Creba J (1990) Leukotriene $\mathrm{D}_{4}$ induced calcium changes in U937 cells may utilize mechanisms additional to inositol phosphate production that are pertussis toxin insensitive but are blocked by phorbol myristate acetate. Cell Signal 2:563-568.

Porreca E, Di Febbo C, Di Sciullo A, Angelucci D, Nasuti M, Vitullo P, Reale M, Conti P, Cuccurullo F, and Poggi A (1996) Cysteinyl leukotriene D4 induced vascular smooth muscle cell proliferation: a possible role in myointimal hyperplasia. Thromb Haemost 76:99-104.

Porreca E, Di Febbo C, Reale M, Barbacane R, Mezzetti A, Cuccurullo F, and Conti $\mathrm{P}$ (1995) Modulation of rat vascular smooth muscle cell (VSMC) proliferation by cysteinyl leukotriene D4: a role for mediation of interleukin 1. Atherosclerosis 113:11-18.

Poulin S, Thompson C, Thivierge M, Véronneau S, McMahon S, Dubois CM, Stankova J, and Rola-Pleszczynski M (2011) Cysteinyl-leukotrienes induce vascular endothelial growth factor production in human monocytes and bronchial smooth muscle cells. Clin Exp Allergy 41:204-217.

Price DB, Swern A, Tozzi CA, Philip G, and Polos P (2006) Effect of montelukast on lung function in asthma patients with allergic rhinitis: analysis from the COMPACT trial. Allergy 61:737-742.

Prinster SC, Hague C, and Hall RA (2005) Heterodimerization of G protein-coupled receptors: specificity and functional significance. Pharmacol Rev 57:289-298.

Profita M, Sala A, Bonanno A, Siena L, Ferraro M, Di Giorgi R, Montalbano AM, Albano GD, Gagliardo R, and Gjomarkaj M (2008) Cysteinyl leukotriene-1 receptor activation in a human bronchial epithelial cell line leads to signal transducer and activator of transcription 1-mediated eosinophil adhesion. J Pharmacol Exp Ther 325:1024-1030.

Przylipiak A, Hafner J, Przylipiak J, Runnebaum B, Rabe T, and Köhn FM (1998) Influence of leukotrienes on in vitro growth of human mammary carcinoma cell line MCF-7. Eur J Obstet Gynecol Reprod Biol 77:61-65.

Pullerits T, Praks L, Ristioja V, and Lötvall J (2002) Comparison of a nasal glucocorticoid, antileukotriene, and a combination of antileukotriene and antihistamine in the treatment of seasonal allergic rhinitis. J Allergy Clin Immunol 109:949955.

Qian XD, Wei EQ, Zhang L, Sheng WW, Wang ML, Zhang WP, and Chen Z (2006) Pranlukast, a cysteinyl leukotriene receptor 1 antagonist, protects mice against brain cold injury. Eur J Pharmacol 549:35-40.

Qiu H, Johansson AS, Sjöström M, Wan M, Schröder O, Palmblad J, and Haeggström JZ (2006) Differential induction of BLT receptor expression on human endothelial cells by lipopolysaccharide, cytokines, and leukotriene B4. Proc Natl Acad Sci USA 103:6913-6918

Queto T, Gaspar-Elsas MI, Masid-de-Brito D, Vasconcelos ZF, Ferraris FK, Penido C, Cunha FQ, Kanaoka Y, Lam BK, and Xavier-Elsas P (2010) Cysteinylleukotriene type 1 receptors transduce a critical signal for the up-regulation of eosinophilopoiesis by interleukin-13 and eotaxin in murine bone marrow. J Leukoc Biol 87:885-893.

Rådmark O and Samuelsson B (2009) 5-Lipoxygenase: mechanisms of regulation. $J$ Lipid Res 50 (Suppl):S40-S45.

Rajah R, Nunn SE, Herrick DJ, Grunstein MM, and Cohen P (1996) Leukotriene D4 induces MMP-1, which functions as an IGFBP protease in human airway smooth muscle cells. Am J Physiol 271:L1014-L1022.

Ravasi S, Citro S, Viviani B, Capra V, and Rovati GE (2006) CysLT1 receptorinduced human airway smooth muscle cells proliferation requires ROS generation, EGF receptor transactivation and ERK1/2 phosphorylation. Respir Res 7:42.
Rediske J, Morrissey MM, and Jarvis M (1993) Human monocytes respond to leukotriene B4 with a transient increase in cytosolic calcium. Cell Immunol 147: $438-445$

Reid DW, Misso N, Aggarwal S, Thompson PJ, and Walters EH (2007) Oxidative stress and lipid-derived inflammatory mediators during acute exacerbations of cystic fibrosis. Respirology 12:63-69.

Reimers A, Pichler C, Helbling A, Pichler WJ, and Yawalkar N (2002) Zafirlukast has no beneficial effects in the treatment of chronic urticaria. Clin Exp Allergy 32:1763-1768.

Riccioni G, Capra V, D’Orazio N, Bucciarelli T, and Bazzano LA (2008) Leukotriene modifiers in the treatment of cardiovascular diseases. J Leukoc Biol 84:13741378.

Riccioni G, Di Ilio C, and D'Orazio N (2004) An update of the leukotriene modulators for the treatment of asthma. Expert Opin Investig Drugs 13:763-776.

Richards IM, Griffin RL, Oostveen JA, Morris J, Wishka DG, and Dunn CJ (1989) Effect of the selective leukotriene B4 antagonist U-75302 on antigen-induced bronchopulmonary eosinophilia in sensitized guinea pigs. Am Rev Respir Dis 140:1712-1716.

Rioux N and Castonguay A (1998) Inhibitors of lipoxygenase: a new class of cancer chemopreventive agents. Carcinogenesis 19:1393-1400.

Rodrigo GJ and Yañez A (2006) The role of antileukotriene therapy in seasonal allergic rhinitis: a systematic review of randomized trials. Ann Allergy Asthmo Immunol 96:779-786.

Rola-Pleszczynski M, Chavaillaz PA, and Lemaire I (1986) Stimulation of interleukin 2 and interferon $\gamma$ production by leukotriene B4 in human lymphocyte cultures. Prostaglandins Leukot Med 23:207-210.

Rola-Pleszczynski M and Lemaire I (1985) Leukotrienes augment interleukin production by human monocytes. J Immunol 135:3958-3961.

Rola-Pleszczynski M and Stanková J (1992) Leukotriene B4 enhances interleukin-6 (IL-6) production and IL-6 messenger RNA accumulation in human monocytes in vitro: transcriptional and posttranscriptional mechanisms. Blood 80:1004-1011.

Rovati GE, Baroffio M, Citro S, Brichetto L, Ravasi S, Milanese M, Crimi E, and Brusasco V (2006) Cysteinyl-leukotrienes in the regulation of $\beta_{2}$-adrenoceptor function: an in vitro model of asthma. Respir Res 7:103.

Rovati GE and Capra V (2007) Cysteinyl-leukotriene receptors and cellular signals ScientificWorldJournal 7:1375-1392.

Rovati GE, Capra V, and Nicosia S (1997) More on the classification of cysteinyl leukotriene receptors. Trends Pharmacol Sci 18:148-149.

Rubinstein I, Kumar B, and Schriever C (2004) Long-term montelukast therapy in moderate to severe COPD—a preliminary observation. Respir Med 98:134-138

Runarsson G, Liu A, Mahshid Y, Feltenmark S, Pettersson A, Klein E, Björkholm M and Claesson HE (2005) Leukotriene B4 plays a pivotal role in CD40-dependent activation of chronic B lymphocytic leukemia cells. Blood 105:1274-1279.

Sabirsh A, Bristulf J, and Owman C (2004) Exploring the pharmacology of the leukotriene B4 receptor BLT1, without the confounding effects of BLT2. Eur $J$ Pharmacol 499:53-65.

Sabirsh A, Bywater RP, Bristulf J, Owman C, and Haeggström JZ (2006) Residues from transmembrane helices 3 and 5 participate in leukotriene B4 binding to BLT1. Biochemistry 45:5733-5744

Saegusa S, Tsubone H, and Kuwahara M (2001) Leukotriene $\mathrm{D}_{4}$-induced Rhomediated actin reorganization in human bronchial smooth muscle cells. Eur $J$ Pharmacol 413:163-171.

Saengpanich S, deTineo M, Naclerio RM, and Baroody FM (2003) Fluticasone nasal spray and the combination of loratadine and montelukast in seasonal allergic rhinitis. Arch Otolaryngol Head Neck Surg 129:557-562.

Saito K, Nagata M, Kikuchi I, and Sakamoto Y (2004) Leukotriene $\mathrm{D}_{4}$ and eosinophil transendothelial migration, superoxide generation, and degranulation via $\beta_{2}$ in tegrin. Ann Allergy Asthma Immunol 93:594-600.

Sakata K, Dahlén SE, and Bäck M (2004) The contractile action of leukotriene $\mathrm{B}_{4}$ in the guinea-pig lung involves a vascular component. Br J Pharmacol 141:449-456.

Sampson AP, Pizzichini E, and Bisgaard H (2003) Effects of cysteinyl leukotrienes and leukotriene receptor antagonists on markers of inflammation. $J$ Allergy Clin Immunol 111:S49-S59; discussion S59-S61.

Sampson AP, Spencer DA, Green CP, Piper PJ, and Price JF (1990) Leukotrienes in the sputum and urine of cystic fibrosis children. Br J Clin Pharmacol 30:861-869.

Samuelsson B (1983) From studies of biochemical mechanism to novel biological mediators: prostaglandin endoperoxides, thromboxanes, and leukotrienes. Nobe Lecture, 8 December 1982. Biosci Rep 3:791-813.

Sanada S, Tanaka T, Kameyoshi Y, and Hide M (2005) The effectiveness of montelukast for the treatment of anti-histamine-resistant chronic urticaria. Arch Der matol Res 297:134-138.

Sánchez-Borges M, Acevedo N, Vergara C, Jiménez S, Zabner-Oziel P, Monzón A and Caraballo L (2009) The A-444C polymorphism in the leukotriene C4 synthase gene is associated with aspirin-induced urticaria. J Investig Allergol Clin Immuno 19:375-382.

Sanz C, Isidro-García M, Dávila I, Moreno E, Laffond E, and Lorente F (2006 Analysis of $927 \mathrm{~T}>\mathrm{C}$ CYSLTRI and $-444 \mathrm{~A}>\mathrm{C}$ LTC4S polymorphisms in patients with asthma. J Investig Allergol Clin Immunol 16:331-337.

Sarau HM, Ames RS, Chambers J, Ellis C, Elshourbagy N, Foley JJ, Schmidt DB Muccitelli RM, Jenkins O, Murdock PR, et al. (1999) Identification, molecula cloning, expression, and characterization of a cysteinyl leukotriene receptor. $\mathrm{Mol}$ Pharmacol 56:657-663.

Sardana N, Santos C, Lehman E, and Craig T (2010) A comparison of intranasal corticosteroid, leukotriene receptor antagonist, and topical antihistamine in reducing symptoms of perennial allergic rhinitis as assessed through the Rhinitis Severity Score. Allergy Asthma Proc 31:5-9.

Saussy DL Jr, Sarau HM, Foley JJ, Mong S, and Crooke ST (1989) Mechanisms of leukotriene $\mathrm{E}_{4}$ partial agonist activity at leukotriene $\mathrm{D}_{4}$ receptors in differentiated U-937 cells. J Biol Chem 264:19845-19855.

Scadding GK, Durham SR, Mirakian R, Jones NS, Drake-Lee AB, Ryan D, Dixon TA Huber PA, Nasser SM, and British Society for Allergy and Clinical Immunology 
(2008) BSACI guidelines for the management of rhinosinusitis and nasal polyposis. Clin Exp Allergy 38:260-275.

Schmitt-Grohé S, Eickmeier O, Naujoks C, Schubert R, Lentze MJ, Zielen S, and Rietschel E (2007) Effects of long-term treatment with montelukast in mild cystic fibrosis (long term treatment with montelukast in cystic fibrosis). Respir Med 101:684.

Schmitt-Grohé S, Eickmeier O, Schubert R, Bez C, and Zielen S (2002) Antiinflammatory effects of montelukast in mild cystic fibrosis. Ann Allergy Asthma Immunol 89:599-605.

Sehmi R, Wardlaw AJ, Cromwell O, Kurihara K, Waltmann P, and Kay AB (1992) Interleukin-5 selectively enhances the chemotactic response of eosinophils obtained from normal but not eosinophilic subjects. Blood 79:2952-2959.

Seo KW, Lee SJ, Kim CE, Yun MR, Park HM, Yun JW, Bae SS, and Kim CD (2010) Participation of 5-lipoxygenase-derived $\mathrm{LTB}_{4}$ in 4-hydroxynonenal-enhanced MMP-2 production in vascular smooth muscle cells. Atherosclerosis 208:56-61.

Serezani CH, Aronoff DM, Sitrin RG, and Peters-Golden M (2009) Fc $\gamma$ RI ligation leads to a complex with BLT1 in lipid rafts that enhances rat lung macrophage antimicrobial functions. Blood 114:3316-3324.

Serhan CN, Chiang N, and Van Dyke TE (2008) Resolving inflammation: dual anti-inflammatory and pro-resolution lipid mediators. Nat Rev Immunol 8:349361.

Serhan CN, Radin A, Smolen JE, Korchak H, Samuelsson B, and Weissmann G (1982) Leukotriene $B_{4}$ is a complete secretagogue in human neutrophils: a kinetic analysis. Biochem Biophys Res Commun 107:1006-1012.

Shao WH, Del Prete A, Bock CB, and Haribabu B (2006) Targeted disruption of leukotriene B4 receptors BLT1 and BLT2: a critical role for BLT1 in collageninduced arthritis in mice. J Immunol 176:6254-6261.

Sharon P and Stenson WF (1984) Enhanced synthesis of leukotriene B4 by colonic mucosa in inflammatory bowel disease. Gastroenterology 86:453-460.

Sheng WW, Li CT, Zhang WP, Yuan YM, Hu H, Fang SH, Zhang L, and Wei EQ (2006) Distinct roles of CysLT1 and CysLT2 receptors in oxygen glucose deprivation-induced PC12 cell death. Biochem Biophys Res Commun 346:19-25.

Shimizu T (2009) Lipid mediators in health and disease: enzymes and receptors as therapeutic targets for the regulation of immunity and inflammation. Annu Rev Pharmacol Toxicol 49:123-150.

Shimizu T, Rådmark O, and Samuelsson B (1984) Enzyme with dual lipoxygenase activities catalyzes leukotriene $\mathrm{A}_{4}$ synthesis from arachidonic acid. Proc Natl Acad Sci USA 81:689-693.

Shin EH, Lee HY, and Bae YS (2006) Leukotriene B4 stimulates human monocytederived dendritic cell chemotaxis. Biochem Biophys Res Commun 348:606-611.

Shin JA, Chang HS, Park SM, Jang AS, Park SW, Park JS, Uh ST, Il Lim G, Rhim T, Kim MK, et al. (2009) Genetic effect of CysLTR2 polymorphisms on its mRNA synthesis and stabilization. BMC Med Genet 10:106.

Shiota N, Shimoura K, and Okunishi H (2006) Pathophysiological role of mast cells in collagen-induced arthritis: study with a cysteinyl leukotriene receptor antagonist, montelukast. Eur J Pharmacol 548:158-166.

Shirasaki H, Kanaizumi E, Seki N, Kikuchi M, Watanabe K, Konno N, and Himi T (2006) Distribution of specific binding sites for cysteinyl leukotriene 1 receptor antagonist in human nasal mucosa. Acta Otolaryngol 126:948-951.

Shirasaki H, Kanaizumi E, Watanabe K, Matsui T, Sato J, Narita S, Rautiainen M, and Himi T (2002) Expression and localization of the cysteinyl leukotriene 1 receptor in human nasal mucosa. Clin Exp Allergy 32:1007-1012.

Shirasaki H, Seki N, Fujita M, Kikuchi M, Kanaizumi E, Watanabe K, and Himi T (2007) Agonist- and $\mathrm{T}_{\mathrm{H}} 2$ cytokine-induced up-regulation of cysteinyl leukotriene receptor messenger RNA in human monocytes. Ann Allergy Asthma Immunol 99:340-347.

Showell HJ, Conklyn MJ, Alpert R, Hingorani GP, Wright KF, Smith MA, Stam E, Salter ED, Scampoli DN, Meltzer S, et al. (1998) The preclinical pharmacological profile of the potent and selective leukotriene B4 antagonist CP-195543. J Pharmacol Exp Ther 285:946-954.

Silverman ES, Liggett SB, Gelfand EW, Rosenwasser LJ, Baron RM, Bolk S, Weiss ST, and Drazen JM (2001) The pharmacogenetics of asthma: a candidate gene approach. Pharmacogenomics $J$ 1:27-37.

Sjölander A, Grönroos E, Hammarström S, and Andersson T (1990) Leukotriene D and $\mathrm{E}_{4}$ induce transmembrane signaling in human epithelial cells. Single cell analysis reveals diverse pathways at the G-protein level for the influx and the intracellular mobilization of $\mathrm{Ca}^{2+}$. J Biol Chem 265:20976-20981.

Sjöström M, Jakobsson PJ, Heimburger M, Palmblad J, and Haeggström JZ (2001) Human umbilical vein endothelial cells generate leukotriene $\mathrm{C} 4$ via microsomal glutathione S-transferase type 2 and express the $\mathrm{CysLT}_{1}$ receptor. Eur J Biochem 268:2578-2586.

Sjöström M, Jakobsson PJ, Juremalm M, Ahmed A, Nilsson G, Macchia L, and Haeggström JZ (2002) Human mast cells express two leukotriene $\mathrm{C}_{4}$ synthase isoenzymes and the CysLT 1 receptor. Biochim Biophys Acta 1583:53-62.

Sjöström M, Johansson AS, Schröder O, Qiu H, Palmblad J, and Haeggström JZ (2003) Dominant expression of the CysLT2 receptor accounts for calcium signaling by cysteinyl leukotrienes in human umbilical vein endothelial cells. Arterioscler Thromb Vasc Biol 23:E37-E41.

Skoglund G and Claesson HE (1991) Intracellular mechanisms involved in leukotriene C4-stimulated adhesion of U-937 cells. Cell Signal 3:399-404.

Sladek K and Szczeklik A (1993) Cysteinyl leukotrienes overproduction and mast cell activation in aspirin-provoked bronchospasm in asthma. Eur Respir $J$ 6:391-399.

Sloniewsky DE, Ridge KM, Adir Y, Fries FP, Briva A, Sznajder JI, and Sporn PH (2004) Leukotriene D4 activates alveolar epithelial Na,K-ATPase and increases alveolar fluid clearance. Am J Respir Crit Care Med 169:407-412.

Smith JR, Subbarao K, Franc DT, Haribabu B, and Rosenbaum JT (2004) Susceptibility to endotoxin induced uveitis is not reduced in mice deficient in BLT1, the high affinity leukotriene $\mathrm{B}_{4}$ receptor. Br J Ophthalmol 88:273-275.

Snyder DS, Castro R, and Desforges JF (1989) Antiproliferative effects of lipoxygenase inhibitors on malignant human hematopoietic cell lines. Exp Hematol 17:6-9.

Song P, Crimi E, Milanese M, Duan J, Rehder K, and Brusasco V (1998) Anti- inflammatory agents and allergen-induced $\beta_{2}$-receptor dysfunction in isolated human bronchi. Am J Respir Crit Care Med 158:1809-1814.

Soter NA, Lewis RA, Corey EJ, and Austen KF (1983) Local effects of synthetic leukotrienes (LTC4, LTD4, LTE4, and LTB4) in human skin. J Invest Dermatol 80:115-119.

Sousa AR, Parikh A, Scadding G, Corrigan CJ, and Lee TH (2002) Leukotrienereceptor expression on nasal mucosal inflammatory cells in aspirin-sensitive rhinosinusitis. $N$ Engl J Med 347:1493-1499.

Spanbroek R, Grabner R, Lotzer K, Hildner M, Urbach A, Ruhling K, Moos MP, Kaiser B, Cohnert TU, Wahlers T, et al. (2003) Expanding expression of the 5-lipoxygenase pathway within the arterial wall during human atherogenesis. Proc Natl Acad Sci USA 100:1238-1243.

Spencer DA, Sampson AP, Green CP, Costello JF, Piper PJ, and Price JF (1992) Sputum cysteinyl-leukotriene levels correlate with the severity of pulmonary disease in children with cystic fibrosis. Pediatr Pulmonol 12:90-94.

Stankova J, Turcotte S, Harris J, and Rola-Pleszczynski M (2002) Modulation of leukotriene B4 receptor-1 expression by dexamethasone: potential mechanism for enhanced neutrophil survival. J Immunol 168:3570-3576.

Steele VE, Holmes CA, Hawk ET, Kopelovich L, Lubet RA, Crowell JA, Sigman CC and Kelloff GJ (1999) Lipoxygenase inhibitors as potential cancer chemopreventives. Cancer Epidemiol Biomarkers Prev 8:467-483.

Steinke JW, Bradley D, Arango P, Crouse CD, Frierson H, Kountakis SE, Kraft M and Borish L (2003) Cysteinyl leukotriene expression in chronic hyperplastic sinusitis-nasal polyposis: importance to eosinophilia and asthma. J Allergy Clin Immunol 111:342-349.

Steinke JW, Crouse CD, Bradley D, Hise K, Lynch K, Kountakis SE, and Borish L (2004) Characterization of interleukin-4-stimulated nasal polyp fibroblasts. Am $J$ Respir Cell Mol Biol 30:212-219.

Stelmach I, Korzeniewska A, Stelmach W, Majak P, Grzelewski T, and Jerzynska J (2005) Effects of montelukast treatment on clinical and inflammatory variables in patients with cystic fibrosis. Ann Allergy Asthma Immunol 95:372-380.

Stelmach R and Cukier A (2006) Treating allergic rhinitis and asthma: different sides of the same fence. Expert Opin Pharmacother 7:1245-1249.

Sterrett S, Morton J, Perry D, and Donovan J (2006) Eosinophilic cystitis: successfu long-term treatment with montelukast sodium. Urology 67:423.e19-423.e21.

Stevenson DD and Simon RA (2001) Lack of cross-reactivity between rofecoxib and aspirin in aspirin-sensitive patients with asthma. J Allergy Clin Immunol 108: $47-51$

Stevenson DD and Szczeklik A (2006) Clinical and pathologic perspectives on aspirin sensitivity and asthma. J Allergy Clin Immunol 118:773-786; quiz 787-788.

Stokes J and Casale TB (2004) Rationale for new treatments aimed at IgE immunomodulation. Ann Allergy Asthma Immunol 93:212-217; quiz 217-219, 271.

Storms W (2007) Update on montelukast and its role in the treatment of asthma, allergic rhinitis and exercise-induced bronchoconstriction. Expert Opin Pharma cother 8:2173-2187.

Strauch E, Moske O, Thoma S, Storm Van's Gravesande K, Thorst G, Brandis M, and Kuehr J (2003) A randomized controlled trial on the effect of montelukast on sputum eosinophil cationic protein in children with corticosteroid-dependent asthma. Pediatr Res 54:198-203.

Subbarao K, Jala VR, Mathis S, Suttles J, Zacharias W, Ahamed J, Ali H, Tseng MT, and Haribabu B (2004) Role of leukotriene B4 receptors in the development of atherosclerosis: potential mechanisms. Arterioscler Thromb Vasc Biol 24:369-375.

Sun FF, Crittenden NJ, Czuk CI, Taylor BM, Stout BK, and Johnson HG (1991) Biochemical and functional differences between eosinophils from animal species and man. J Leukoc Biol 50:140-150.

Sun R, Ba X, Cui L, Xue Y, and Zeng X (2009) Leukotriene B4 regulates proliferation and differentiation of cultured rat myoblasts via the BLT1 pathway. Mol Cells 27:403-408

Suzuki M, Kato M, Kimura H, Fujiu T, and Morikawa A (2003) Inhibition of human eosinophil activation by a cysteinyl leukotriene receptor antagonist (pranlukast; ONO-1078). J Asthma 40:395-404

Suzuki S, Takeuchi K, Ishinaga H, Basbaum C, and Majima Y (2008) Leukotriene D4 upregulates MUC2 gene transcription in human epithelial cells. Pharmacology 81:221-228.

Szczeklik A, Sladek K, Dworski R, Nizankowska E, Soja J, Sheller J, and Oates J (1996) Bronchial aspirin challenge causes specific eicosanoid response in aspirinsensitive asthmatics. Am J Respir Crit Care Med 154:1608-1614

Szczeklik A and Stevenson DD (2003) Aspirin-induced asthma: advances in pathogenesis, diagnosis, and management. J Allergy Clin Immunol 111:913-921; quiz 922.

Tager AM, Bromley SK, Medoff BD, Islam SA, Bercury SD, Friedrich EB, Carafone $\mathrm{AD}$, Gerszten RE, and Luster AD (2003) Leukotriene $\mathrm{B}_{4}$ receptor BLT1 mediates early effector T cell recruitment. Nat Immunol 4:982-990.

Tager AM, Dufour JH, Goodarzi K, Bercury SD, von Andrian UH, and Luster AD (2000) BLTR mediates leukotriene $\mathrm{B}_{4}$-induced chemotaxis and adhesion and plays a dominant role in eosinophil accumulation in a murine model of peritonitis. J Exp Med 192:439-446.

Tahan F, Jazrawi E, Moodley T, Rovati GE, and Adcock IM (2008) Montelukast inhibits tumour necrosis factor- $\alpha$-mediated interleukin- 8 expression through inhibition of nuclear factor- $\kappa$ B p65-associated histone acetyltransferase activity. Clin Exp Allergy 38:805-811.

Takamatsu Y, Shimada K, Chijiiwa K, Kuroki S, Yamaguchi K, and Tanaka M (2004) Role of leukotrienes on hepatic ischemia/reperfusion injury in rats. J Surg Res 119: $14-20$

Takasaki J, Kamohara M, Matsumoto M, Saito T, Sugimoto T, Ohishi T, Ishii H, Ota T, Nishikawa T, Kawai Y, et al. (2000) The molecular characterization and tissue distribution of the human cysteinyl leukotriene $\mathrm{CysLT}_{2}$ receptor. Biochem Biophys Res Commun 274:316-322.

Tanimoto Y, Takahashi K, and Kimura I (1992) Effects of cytokines on human basophil chemotaxis. Clin Exp Allergy 22:1020-1025.

Tantisira KG, Lima J, Sylvia J, Klanderman B, and Weiss ST (2009) 5-lipoxygenase 
pharmacogenetics in asthma: overlap with Cys-leukotriene receptor antagonist loci. Pharmacogenet Genomics 19:244-247.

Taylor-Clark TE, Nassenstein C, and Undem BJ (2008) Leukotriene $\mathrm{D}_{4}$ increases the excitability of capsaicin-sensitive nasal sensory nerves to electrical and chemical stimuli. Br J Pharmacol 154:1359-1368.

Tedeschi A (2009) Paradoxical exacerbation of chronic urticaria by H1-antihistamines and montelukast. Eur Ann Allergy Clin Immunol 41:187-189.

Telleria JJ, Blanco-Quiros A, Varillas D, Armentia A, Fernandez-Carvajal I, Jesus Alonso M, and Diez I (2008) ALOX5 promoter genotype and response to montelukast in moderate persistent asthma. Respir Med 102:857-861.

Terawaki K, Yokomizo T, Nagase T, Toda A, Taniguchi M, Hashizume K, Yagi T, and Shimizu T (2005) Absence of leukotriene $\mathrm{B}_{4}$ receptor 1 confers resistance to airway hyperresponsiveness and Th2-type immune responses. J Immunol 175:42174225.

Thivierge M, Doty M, Johnson J, Stanková J, and Rola-Pleszczynski M (2000) IL-5 up-regulates cysteinyl leukotriene 1 receptor expression in HL-60 cells differentiated into eosinophils. J Immunol 165:5221-5226.

Thivierge M, Stanková J, and Rola-Pleszczynski M (2001) IL-13 and IL-4 up-regulate cysteinyl leukotriene 1 receptor expression in human monocytes and macrophages. Immunol 167:2855-2860.

Thivierge M, Stankova J, and Rola-Pleszczynski M (2006) Toll-like receptor agonists differentially regulate cysteinyl-leukotriene receptor 1 expression and function in human dendritic cells. J Allergy Clin Immunol 117:1155-1162.

Thivierge M, Stankova J, and Rola-Pleszczynski M (2009) Cysteinyl-leukotriene receptor type 1 expression and function is down-regulated during monocytederived dendritic cell maturation with zymosan: involvement of IL-10 and prostaglandins. J Immunol 183:6778-6787.

Thodeti CK, Adolfsson J, Juhas M, and Sjölander A (2000) Leukotriene $\mathrm{D}_{4}$ triggers an association between $\mathrm{G} \beta \gamma$ subunits and phospholipase C- $\gamma 1$ in intestinal epithelial cells. J Biol Chem 275:9849-9853.

Thodeti CK, Nielsen CK, Paruchuri S, Larsson C, and Sjölander A (2001) The 8 isoform of protein kinase $\mathrm{C}$ is involved in regulation of the $\mathrm{LTD}_{4}$-induced calcium signal in human intestinal epithelial cells. Exp Cell Res 262:95-103.

Thomas M (2006) Allergic rhinitis: evidence for impact on asthma. BMC Pulm Med 6 (Suppl 1):S4.

Thompson C, Cloutier A, Bossé Y, Poisson C, Larivée P, McDonald PP, Stankova J, and Rola-Pleszczynski M (2008a) Signaling by the cysteinyl-leukotriene receptor 2 . Involvement in chemokine gene transcription. J Biol Chem 283:1974-1984.

Thompson C, Cloutier A, Bossé Y, Thivierge M, Gouill CL, Larivée P, McDonald PP, Stankova J, and Rola-Pleszczynski M (2006) CysLT1 receptor engagement induces activator protein-1- and NF- $\kappa \mathrm{B}-d e p e n d e n t$ IL-8 expression. Am J Respir Cell Mol Biol 35:697-704

Thompson C, McMahon S, Bossé Y, Dubois CM, Stankova J, and Rola-Pleszczynski M (2008b) Leukotriene $\mathrm{D}_{4}$ upregulates furin expression through CysLT1 receptor signaling. Am J Respir Cell Mol Biol 39:227-234

Thompson MD, Burnham WM, and Cole DE (2005) The G protein-coupled receptors: pharmacogenetics and disease. Crit Rev Clin Lab Sci 42:311-392.

Thompson MD, Capra V, Takasaki J, Maresca G, Rovati GE, Slutsky AS, Lilly C, Zamel N, McIntyre Burnham W, Cole DE, et al. (2007) A functional G300S variant of the cysteinyl leukotriene 1 receptor is associated with atopy in a Tristan da Cunha isolate. Pharmacogenet Genomics 17:539-549.

Thompson MD, Storm van's Gravesande K, Galczenski H, Burnham WM, Siminovitch KA, Zamel N, Slutsky A, Drazen JM, George SR, Evans JF, et al. (2003) A cysteinyl leukotriene 2 receptor variant is associated with atopy in the population of Tristan da Cunha. Pharmacogenetics 13:641-649.

Titos E, Clària J, Planagumà A, López-Parra M, González-Périz A, Gaya J, Miquel R, Arroyo V, and Rodés J (2005) Inhibition of 5-lipoxygenase-activating protein abrogates experimental liver injury: role of Kupffer cells. J Leukoc Biol 78:871878 .

Toda A, Terawaki K, Yamazaki S, Saeki K, Shimizu T, and Yokomizo T (2010) Attenuated Th1 induction by dendritic cells from mice deficient in the leukotriene B4 receptor 1. Biochimie 92:682-691.

Toda A, Yokomizo T, Masuda K, Nakao A, Izumi T, and Shimizu T (1999) Cloning and characterization of rat leukotriene $\mathrm{B}_{4}$ receptor. Biochem Biophys Res Commun 262:806-812.

Toki Y, Hieda N, Torii T, Hashimoto H, Ito T, Ogawa K, and Satake T (1988) The effects of lipoxygenase inhibitor and peptidoleukotriene antagonist on myocardial injury in a canine coronary occlusion-reperfusion model. Prostaglandins 35:555571.

Tomari S, Matsuse H, Machida I, Kondo Y, Kawano T, Obase Y, Fukushima C, Shimoda T, and Kohno S (2003) Pranlukast, a cysteinyl leukotriene receptor 1 antagonist, attenuates allergen-specific tumour necrosis factor $\alpha$ production and nuclear factor $\kappa \mathrm{B}$ nuclear translocation in peripheral blood monocytes from atopic asthmatics. Clin Exp Allergy 33:795-801.

Tong WG, Ding XZ, Talamonti MS, Bell RH, and Adrian TE (2005) LTB4 stimulates growth of human pancreatic cancer cells via MAPK and PI-3 kinase pathways. Biochem Biophys Res Commun 335:949-956.

Tornhamre S, Stenke L, Granzelius A, Sjölinder M, Näsman-Glaser B, Roos C, Widell S, and Lindgren JA (2003) Inverse relationship between myeloid maturation and leukotriene $\mathrm{C}_{4}$ synthase expression in normal and leukemic myelopoiesisconsistent overexpression of the enzyme in myeloid cells from patients with chronic myeloid leukemia. Exp Hematol 31:122-130.

Trandafir CC, Nishihashi T, Ji X, Wang A, and Kurahashi K (2005) Cysteinyl leukotrienes and leukotriene B mediate vasoconstriction to arginine vasopressin in rat basilar artery. Clin Exp Pharmacol Physiol 32:1027-1033.

Tryselius Y, Nilsson NE, Kotarsky K, Olde B, and Owman C (2000) Cloning and characterization of cDNA encoding a novel human leukotriene $\mathrm{B}_{4}$ receptor. Biochem Biophys Res Commun 274:377-382.

Turner CR, Breslow R, Conklyn MJ, Andresen CJ, Patterson DK, Lopez-Anaya A Owens B, Lee P, Watson JW, and Showell HJ (1996) In vitro and in vivo effects of leukotriene $\mathrm{B}_{4}$ antagonism in a primate model of asthma. J Clin Invest 97:381387.

Ueda T, Takeno S, Furukido K, Hirakawa K, and Yajin K (2003) Leukotriene receptor antagonist pranlukast suppresses eosinophil infiltration and cytokine production in human nasal mucosa of perennial allergic rhinitis. Ann Otol Rhino Laryngol 112:955-961.

Underwood DC, Osborn RR, Newsholme SJ, Torphy TJ, and Hay DW (1996) Persistent airway eosinophilia after leukotriene (LT) D4 administration in the guinea pig: modulation by the LTD4 receptor antagonist, pranlukast, or an interleukin-5 monoclonal antibody. Am J Respir Crit Care Med 154:850-857.

Usery JB, Self TH, Muthiah MP, and Finch CK (2008) Potential role of leukotriene modifiers in the treatment of chronic obstructive pulmonary disease. Pharmacotherapy 28:1183-1187.

Uzonyi B, Lötzer K, Jahn S, Kramer C, Hildner M, Bretschneider E, Radke D, Beer M, Vollandt R, Evans JF, et al. (2006) Cysteinyl leukotriene 2 receptor and protease-activated receptor 1 activate strongly correlated early genes in human endothelial cells. Proc Natl Acad Sci USA 103:6326-6331.

Vaddi K and Newton RC (1994) Regulation of monocyte integrin expression by $\beta$-family chemokines. J Immunol 153:4721-4732.

van Adelsberg J, Philip G, Pedinoff AJ, Meltzer EO, Ratner PH, Menten J, Reiss TF, and Montelukast Fall Rhinitis Study Group (2003) Montelukast improves symptoms of seasonal allergic rhinitis over a 4-week treatment period. Allergy 58:1268 1276

Van Hoecke H, Vandenbulcke L, and Van Cauwenberge P (2007) Histamine and leukotriene receptor antagonism in the treatment of allergic rhinitis: an update Drugs 67:2717-2726.

Vannella KM, McMillan TR, Charbeneau RP, Wilke CA, Thomas PE, Toews GB, Peters-Golden M, and Moore BB (2007) Cysteinyl leukotrienes are autocrine and paracrine regulators of fibrocyte function. J Immunol 179:7883-7890.

Vaquerizo MJ, Casan P, Castillo J, Perpiña M, Sanchis J, Sobradillo V, Valencia A Verea H, Viejo JL, Villasante C, et al. (2003) Effect of montelukast added to inhaled budesonide on control of mild to moderate asthma. Thorax 58:204-210.

Vegesna RV, Wu HL, Mong S, and Crooke ST (1988) Staurosporine inhibits protein kinase $\mathrm{C}$ and prevents phorbol ester-mediated leukotriene $\mathrm{D}_{4}$ receptor desensitization in RBL-1 cells. Mol Pharmacol 33:537-542.

Veien NK, Busch-Sørensen M, and Stausbøl-Grøn B (2005) Montelukast treatment of moderate to severe atopic dermatitis in adults: a randomized, double-blind placebo-controlled trial. J Am Acad Dermatol 53:147-149.

Virchow JC Jr, Prasse A, Naya I, Summerton L, and Harris A (2000) Zafirlukast improves asthma control in patients receiving high-dose inhaled corticosteroids. Am J Respir Crit Care Med 162:578-585.

Virchow JC and Bachert C (2006) Efficacy and safety of montelukast in adults with asthma and allergic rhinitis. Respir Med 100:1952-1959.

Virchow JC Jr, Faehndrich S, Nassenstein C, Bock S, Matthys H, and Luttmann W (2001) Effect of a specific cysteinyl leukotriene-receptor 1-antagonist (montelukast) on the transmigration of eosinophils across human umbilical vein endothelia cells. Clin Exp Allergy 31:836-844.

Wada K, Arita M, Nakajima A, Katayama K, Kudo C, Kamisaki Y, and Serhan CN (2006) Leukotriene $\mathrm{B}_{4}$ and lipoxin $\mathrm{A}_{4}$ are regulatory signals for neural stem cel proliferation and differentiation. FASEB J 20:1785-1792.

Wahn U and Dass SB (2008) Review of recent results of montelukast use as a monotherapy in children with mild asthma. Clin Ther 30 (Spec No):1026-1035.

Walch L, Norel X, Bäck M, Gascard JP, Dahlén SE, and Brink C (2002) Pharmacological evidence for a novel cysteinyl-leukotriene receptor subtype in human pulmonary artery smooth muscle. Br J Pharmacol 137:1339-1345.

Walch L, Norel X, Gascard JP, and Brink C (2000) Functional studies of leukotriene receptors in vascular tissues. Am J Respir Crit Care Med 161:S107-S111.

Wan KS (2009) Efficacy of leukotriene receptor antagonist with an anti-H1 recepto antagonist for treatment of chronic idiopathic urticaria. J Dermatolog Treat 20 $194-197$

Wan M, Sabirsh A, Wetterholm A, Agerberth B, and Haeggström JZ (2007) Leukotriene $B_{4}$ triggers release of the cathelicidin LL-37 from human neutrophils: nove lipid-peptide interactions in innate immune responses. FASEB J 21:2897-2905.

Wang D and Dubois RN (2010a) Eicosanoids and cancer. Nat Rev Cancer 10:181193.

Wang D and Dubois RN (2010b) Eicosanoids and cancer. Nat Rev Cancer 10:181193.

Wang ML, Huang XJ, Fang SH, Yuan YM, Zhang WP, Lu YB, Ding Q, and Wei EQ (2006) Leukotriene $\mathrm{D}_{4}$ induces brain edema and enhances CysLT $\mathrm{T}_{2}$ receptormediated aquaporin 4 expression. Biochem Biophys Res Commun 350:399-404.

Watanabe S, Yamasaki A, Hashimoto K, Shigeoka Y, Chikumi H, Hasegawa Y, Sumikawa T, Takata M, Okazaki R, Watanabe M, et al. (2009) Expression of functional leukotriene $B$ receptors on human airway smooth muscle cells. $J$ Allergy Clin Immunol 124:59-65.e1-3.

Weller CL, Collington SJ, Brown JK, Miller HR, Al-Kashi A, Clark P, Jose PJ, Hartnell A, and Williams TJ (2005) Leukotriene $\mathrm{B}_{4}$, an activation product of mast cells, is a chemoattractant for their progenitors. J Exp Med 201:1961-1971.

Wenzel SE, Trudeau JB, Kaminsky DA, Cohn J, Martin RJ, and Westcott JY (1995) Effect of 5-lipoxygenase inhibition on bronchoconstriction and airway inflammation in nocturnal asthma. Am $J$ Respir Crit Care Med 152:897-905.

Whitney LW, Ludwin SK, McFarland HF, and Biddison WE (2001) Microarray analysis of gene expression in multiple sclerosis and EAE identifies 5-lipoxygenase as a component of inflammatory lesions. J Neuroimmunol 121:40-48.

Wikström K, Juhas M, and Sjölander A (2003a) The anti-apoptotic effect of leukotriene $\mathrm{D}_{4}$ involves the prevention of caspase 8 activation and Bid cleavage. Biochem J 371:115-124.

Wikström K, Ohd JF, and Sjölander A (2003b) Regulation of leukotriene-dependent induction of cyclooxygenase-2 and Bcl-2. Biochem Biophys Res Commun 302:330 335.

Wilson AM, O'Byrne PM, and Parameswaran K (2004) Leukotriene receptor antag- 
onists for allergic rhinitis: a systematic review and meta-analysis. Am J Med 116:338-344.

Winkler JD, Sarau HM, Foley JJ, and Crooke ST (1988) Phorbol 12-myristate 13-acetate inhibition of leukotriene D4-induced signal transduction was rapidly reversed by staurosporine. Biochem Biophys Res Commun 157:521-529.

Winkler JD, Sarau HM, Foley JJ, and Crooke ST (1990) Inhibitors of protein kinase $\mathrm{C}$ selectively enhanced leukotriene D4-induced calcium mobilization in differentiated U-937 cells. Cell Signal 2:427-437.

Woolhouse IS, Bayley DL, and Stockley RA (2002) Sputum chemotactic activity in chronic obstructive pulmonary disease: effect of $\alpha_{1}$-antitrypsin deficiency and the role of leukotriene $\mathrm{B}_{4}$ and interleukin 8. Thorax 57:709-714.

Woszczek G, Chen LY, Alsaaty S, Nagineni S, and Shelhamer JH (2010) Concentration-dependent noncysteinyl leukotriene type 1 receptor-mediated inhibitory activity of leukotriene receptor antagonists. J Immunol 184:2219-2225

Woszczek G, Chen LY, Nagineni S, Alsaaty S, Harry A, Logun C, Pawliczak R, and Shelhamer JH (2007) IFN- $\gamma$ induces cysteinyl leukotriene receptor 2 expression and enhances the responsiveness of human endothelial cells to cysteinyl leukotrienes. J Immunol 178:5262-5270.

Woszczek G, Chen LY, Nagineni S, Kern S, Barb J, Munson PJ, Logun C, Danner RL, and Shelhamer $\mathrm{JH}$ (2008a) Leukotriene $\mathrm{D}_{4}$ induces gene expression in human monocytes through cysteinyl leukotriene type I receptor. J Allergy Clin Immunol 121:215-221.e1.

Woszczek G, Chen LY, Nagineni S, and Shelhamer JH (2008b) IL-10 inhibits cysteinyl leukotriene-induced activation of human monocytes and monocyte-derived dendritic cells. J Immunol 180:7597-7603.

Woszczek G, Pawliczak R, Qi HY, Nagineni S, Alsaaty S, Logun C, and Shelhamer JH (2005) Functional characterization of human cysteinyl leukotriene 1 receptor gene structure. J Immunol 175:5152-5159.

Wu Y, Zhou C, Tao J, and Li S (2006) Montelukast prevents the decrease of interleukin-10 and inhibits NF- $\kappa \mathrm{B}$ activation in inflammatory airway of asthmatic guinea pigs. Can J Physiol Pharmacol 84:531-537.

Wunder F, Tinel H, Kast R, Geerts A, Becker EM, Kolkhof P, Hütter J, Ergüden J and Härter M (2010) Pharmacological characterization of the first potent and selective antagonist at the cysteinyl leukotriene $2\left(\mathrm{CysLT}_{2}\right)$ receptor. $\mathrm{Br} J$ Pharmacol 160:399-409.

Xu ZZ, Zhang L, Liu T, Park JY, Berta T, Yang R, Serhan CN, and Ji RR (2010) Resolvins RvE1 and RvD1 attenuate inflammatory pain via central and peripheral actions. Nat Med 16:592-597.

Yamaoka KA, Claésson HE, and Rosén A (1989) Leukotriene B4 enhances activation, proliferation, and differentiation of human B lymphocytes. J Immunol 143:19962000 .

Yamaoka KA, Dugas B, Paul-Eugene N, Mencia-Huerta JM, Braquet P, and Kolb JP (1994) Leukotriene $B_{4}$ enhances IL-4-induced IgE production from normal human lymphocytes. Cell Immunol 156:124-134.

Yamaoka KA and Kolb JP (1993) Leukotriene $\mathrm{B}_{4}$ induces interleukin 5 generation from human T lymphocytes. Eur J Immunol 23:2392-2398.

Yang G, Haczku A, Chen H, Martin V, Galczenski H, Tomer Y, Van Besien CR, Evans JF, Panettieri RA, Funk CD, et al. (2004) Transgenic smooth muscle expression of the human CysLT1 receptor induces enhanced responsiveness of murine airways to leukotriene D4. Am J Physiol Lung Cell Mol Physiol 286:L992L1001.

Yasuda D, Okuno T, Yokomizo T, Hori T, Hirota N, Hashidate T, Miyano M, Shimizu $\mathrm{T}$, and Nakamura M (2009) Helix 8 of leukotriene B4 type-2 receptor is required for the folding to pass the quality control in the endoplasmic reticulum. FASEB $J$ 23:1470-1481.
Yokomizo T, Izumi T, Chang K, Takuwa Y, and Shimizu T (1997) A G-proteincoupled receptor for leukotriene $\mathrm{B}_{4}$ that mediates chemotaxis. Nature 387:620624.

Yokomizo T, Izumi T, and Shimizu T (2001a) Co-expression of two LTB4 receptors in human mononuclear cells. Life Sci 68:2207-2212.

Yokomizo T, Izumi T, and Shimizu T (2001b) Leukotriene B4: metabolism and signal transduction. Arch Biochem Biophys 385:231-241.

Yokomizo T, Kato K, Hagiya H, Izumi T, and Shimizu T (2001c) Hydroxyeicosanoids bind to and activate the low affinity leukotriene $\mathrm{B}_{4}$ receptor, BLT2. J Biol Chem 276:12454-12459.

Yokomizo T, Kato K, Terawaki K, Izumi T, and Shimizu T (2000) A second leukotriene $\mathrm{B}_{4}$ receptor, BLT2. A new therapeutic target in inflammation and immunological disorders. J Exp Med 192:421-432.

Yoshisue H, Kirkham-Brown J, Healy E, Holgate ST, Sampson AP, and Davies DE (2007) Cysteinyl leukotrienes synergize with growth factors to induce proliferation of human bronchial fibroblasts. J Allergy Clin Immunol 119:132-140.

Yu GL, Wei EQ, Wang ML, Zhang WP, Zhang SH, Weng JQ, Chu LS, Fang SH, Zhou Y, Chen Z, et al. (2005a) Pranlukast, a cysteinyl leukotriene receptor-1 antagonist, protects against chronic ischemic brain injury and inhibits the glial scar formation in mice. Brain Res 1053:116-125.

Yu GL, Wei EQ, Zhang SH, Xu HM, Chu LS, Zhang WP, Zhang Q, Chen Z, Mei RH and Zhao MH (2005b) Montelukast, a cysteinyl leukotriene receptor-1 antagonist, dose- and time-dependently protects against focal cerebral ischemia in mice. Pharmacology 73:31-40.

Yuan YM, Fang SH, Qian XD, Liu LY, Xu LH, Shi WZ, Zhang LH, Lu YB, Zhang WP, and Wei EQ (2009) Leukotriene $\mathrm{D}_{4}$ stimulates the migration but not proliferation

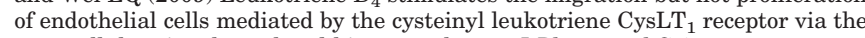
extracellular signal-regulated kinase pathway. J Pharmacol Sci 109:285-292.

Zembowicz A, Mastalerz L, Setkowicz M, Radziszewski W, and Szczeklik A (2003) Safety of cyclooxygenase 2 inhibitors and increased leukotriene synthesis in chronic idiopathic urticaria with sensitivity to nonsteroidal anti-inflammatory drugs. Arch Dermatol 139:1577-1582.

Zhang J, Migita O, Koga M, Shibasaki M, Arinami T, and Noguchi E (2006) Determination of structure and transcriptional regulation of CYSLTR1 and an association study with asthma and rhinitis. Pediatr Allergy Immunol 17:242-249.

Zhang WP, Hu H, Zhang L, Ding W, Yao HT, Chen KD, Sheng WW, Chen Z, and Wei $\mathrm{EQ}$ (2004) Expression of cysteinyl leukotriene receptor 1 in human traumatic brain injury and brain tumors. Neurosci Lett 363:247-251.

Zhao L, Moos MP, Gräbner R, Pédrono F, Fan J, Kaiser B, John N, Schmidt S, Spanbroek R, Lötzer K, et al. (2004) The 5-lipoxygenase pathway promotes pathogenesis of hyperlipidemia-dependent aortic aneurysm. Nat Med 10:966-973.

Zhou G, Zhou Z, Ge S, Liu D, Zhang R, Xu G, Zhu W, Yin Q, Chen AF, and Liu X (2009) IL-18 accelerates the cell apoptosis by up-regulating cysteinyl leukotriene 2 receptor expression in human umbilical vein endothelial cells at the early stage of administration. Vascul Pharmacol 50:171-177.

Zhu J, Qiu YS, Figueroa DJ, Bandi V, Galczenski H, Hamada K, Guntupalli KK, Evans JF, and Jeffery PK (2005) Localization and upregulation of cysteinyl leukotriene-1 receptor in asthmatic bronchial mucosa. Am J Respir Cell Mol Biol 33:531-540.

Zijlstra FJ, van Dijk AP, Garrelds IM, Ouwendijk RJ, and Wilson JH (1992) Species differences in the pattern of eicosanoids produced by inflamed and non-inflamed tissue. Agents Actions Spec No:C73-C75.

Zijlstra FJ, van Dijk AP, Ouwendijk RJ, van Riemsdijk-Overbeeke IC, and Wilson JH (1993) Eicosanoid production by the mucosa in inflammatory bowel disease after 5-ASA treatment. Agents Actions 38 (Spec No):C122-C124. 